\title{
Geomorphologische Untersuchungen mittels GIS- und Fernerkundungsverfahren unter Berücksichtigung hydrogeologischer Fragestellungen - Fallbeispiele aus Nordwest Syrien -
}

\author{
Dissertation \\ zur Erlangung des Doktorgrades \\ der Mathematisch-Naturwissenschaftlichen Fakultäten \\ der Georg-August-Universität zu Göttingen \\ vorgelegt von \\ Wahib Sahwan \\ aus Taybat Al Imam/Syrien
}

Göttingen 2007 
D 7

Referent: $\quad$ Prof. Dr. M. Kappas

Korreferent: $\quad$ Prof. Dr. M. Sauter

Tag der mündlichen Prüfung: 15.01.2008 


\section{Motivation}

Das Untersuchungsgebiet liegt im semi-ariden Nordwesten Syriens und umfasst die ganze Provinz von Aleppo und große Teile vier anderer Hauptprovinzen Syriens (Idlib, Hama, Homs und Raqqa). Im Hinblick auf die Bevölkerungsanzahl zählen diese zu den größten Provinzen Syriens. Aufgrund dessen wird dieses Gebiet intensiv landwirtschaftlich genutzt, welches zur Übernutzung von Grundwasserressourcen und Landdegradierung führte.

In den letzten 20 Jahren nahmen diese Probleme, aufgrund der Klimaveränderung, insbesondere des verringerten Niederschlags, drastisch zu. Daher erfordert diese Lage eine umfassendes Wassermanagment bzw. eine gründliche Beobachtung des geoökologischen Systems. Die Geomorphologie in diesem Gebiet, obwohl sie ein wichtiger Faktor ist und in starker Wechselbeziehung mit dem geoökologischen System steht, bisher sehr wenig betrachtet. Hinzu kommt die mangelnde Verfügbarkeit der digitalen Daten in diesem Gebiet.

Dadurch entstand die Idee zu der vorlegenden Arbeit, fernerkundliche Daten (Landsat und ASTER) in Kombination mit digitalen Geländemodellen geomorphologisch/geomorphometrisch zu analysieren und morphostrukturell zu interpretieren. Die Analyse zielt darauf ab, das Wissen über das geomorphologische Bild des Gebiets zu vervollständigen und Datengrundlage für eine Datenbank zu erstellen. Anschließend lenkte die Arbeit in Richtung der Erstellung einer integrierten hydrogeomorphologischen Datenbank, die als Datengrundlage für weitere Untersuchungen dienen soll.

Schlüsselworte: Geomorphologie, Das digitale Höhenmodell, Fernerkundung, GIS, Hydrogeologie, Syrien.

Key words: Geomorphology, Digital Elevation Model, Remote Sensing, GIS, Hydrogeology, Syria. 


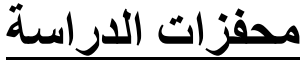

تقع منطقة الدر اسة في شمال غرب سوريا حيث المناخ شبه جاف و تتضمن كامل محافظة حلب و أجزاء كبيرة من أربع محافظات رئيسة أخرى (إذلب و حماة و حمص و الرقة)، من حيث عدد السكان تتضوي هذه المحافظات ضمن أكبر محافظات سوريا. لهذه الأسباب يتم استثمار هذه الدنطقة بشكل كبير جدا، مما يؤدي للاستخدام الجائر لمصادر المياه الجوفية و تدهور النظام البيئي. في السنوات العشرين الأخيرة تفاقت هذه المشاكل بشكل كبير جداو ذلك نتيجة التغير ات المناخية

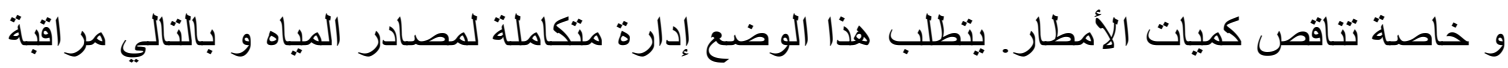

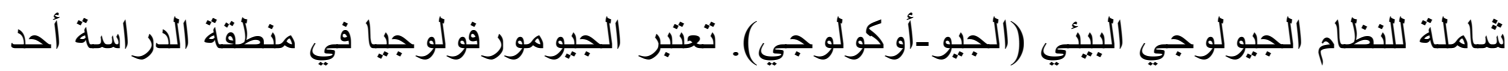

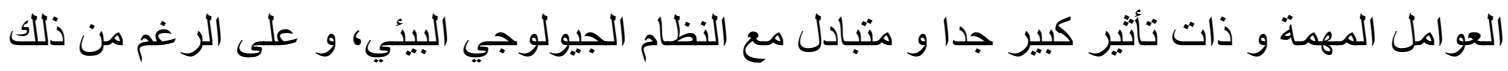
فإنها لم تتمتع حتى الآن بالقدر الكافي من الاهتمام العلمي. يضاف إلى ذلك نقص البيانات الرقمية

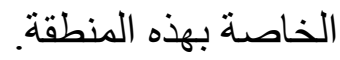

استنادا لهذا الوضع جاءت أفكار هذه الدراسة بأن يتم استخدام بيانات الاستشعار عن بعد (من التابع

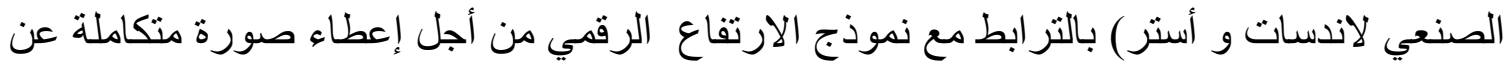

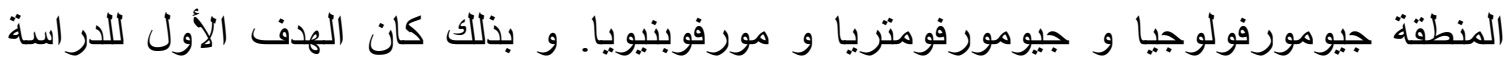
توصيف متكامل لجيومورفولوجيا المنطقة قيد الدراسة و استتباط بيانات لتكوين قاعدة بيانات رقمية. و في النهاية أخذ العمل منحى إنتاج قاعدة بيانات هيدروجيومورفوفولوجية رقمية يمكن أن تكون قاعدة تأسيسية لخدمة در اسات أخرى.

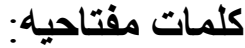

جيومورفولوجيا، نموذج الارتفاع الرقمي، الاستشعار عن بعد، نظام المسح الجغرافي،

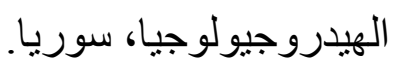




\section{Widmung}

Diese Arbeit möchte ich meinem Heimatland Syrien, meinen Kollegen und Dozenten

in meinem ersten Studium sowie meinen Betreuern und Kollegen des

Geowissenschaftlichen Zentrums der Universität Göttingen

widmen.

Weiterhin möchte ich diese Arbeit meinen Eltern, meiner Frau und

meinen Kindern Raghad, Rahaf und Mohammed

widmen.

$$
\begin{aligned}
& \text { إلم بـلـدي سوريـا } \\
& \text { إلى زمـلائـي و أسـاتـذتـي في مـر احل در استي الأولى } \\
& \text { إلى زمـلائسي و المـشرفـن عـلـي في مـركز علـوم الأرض في جـامـــة } \\
& \text { جـوتـنـنـن } \\
& \text { إلم و الـدي و زوجتي و أبـنـائـي رغد \& ر هف \& عحمـد }
\end{aligned}
$$

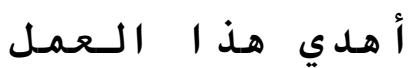

Wahib Ahmad Sahwan

و هيـب أحمد سهو ان 



\section{I nhaltsverzeichnis}

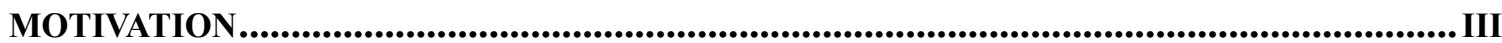

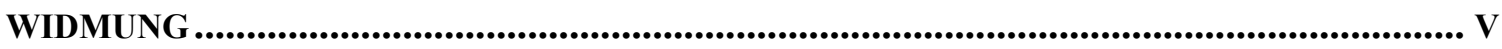

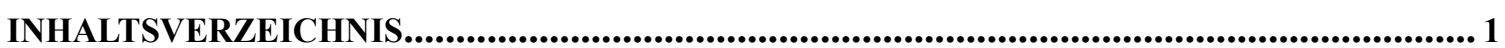

ABBILDUNGSVERZEICHNIS ............................................................................................ II

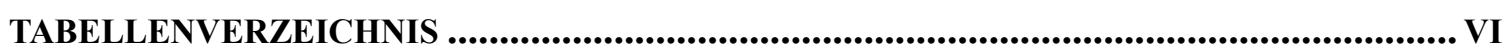

ABKÜRZUNGSVERZEICHNIS................................................................................VIII

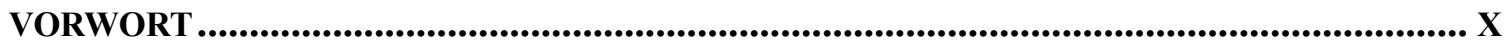

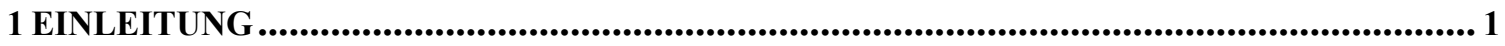

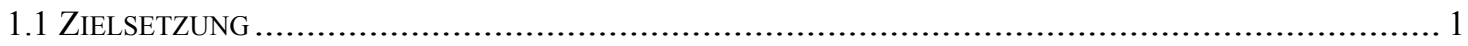

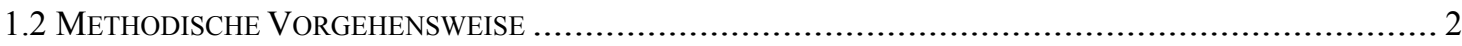

2 CHARAKTERISIERUNG DES UNTERSUCHUNGSGEBIETES ............................................. 5

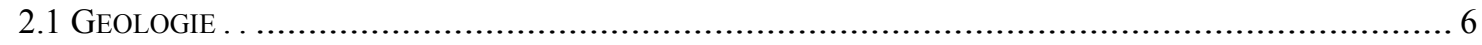

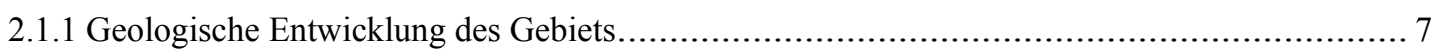

2.1.2 Die paläogeographische Entwicklung der Landschaft.................................................... 9

2.1.3 Die Paläogeographie des Euphrat und Orontes........................................................... 12

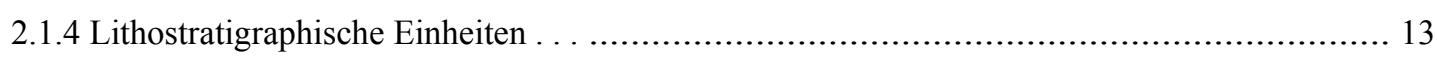

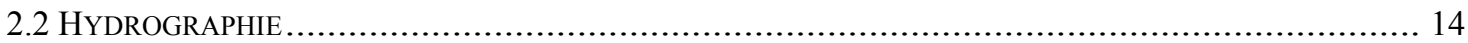

2.3 HYDROGEOLOGIE

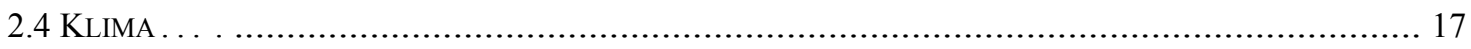

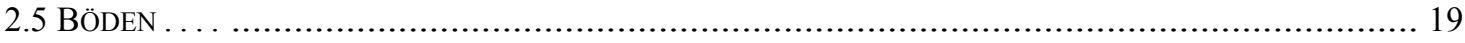

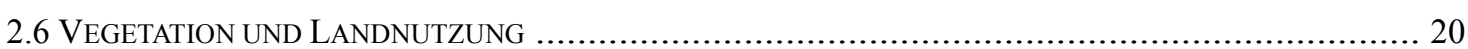

3 FERNERKUNDUNGS-MISSIONEN …...................................................................................... 25

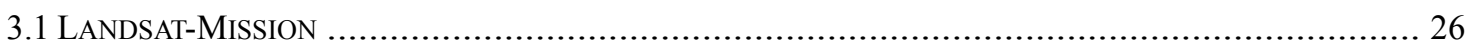

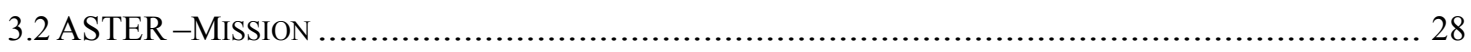

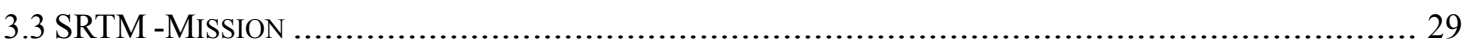

3.4 EINSATZ VON FERNERKUNDUNGSDATEN IN DEN GEOWISSENSCHAFTEN ..................................... 33

4 DATENGRUNDLAGE UND DIE DIGITALE AUFBEREITUNG......................................... 39

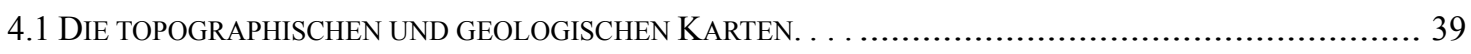

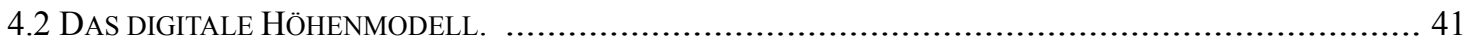

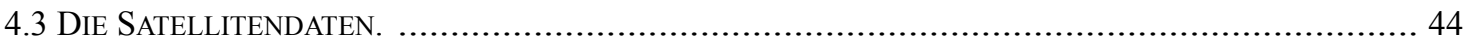


4.3.1 Bildvorbearbeitung (Pre-Processing) .................................................... 45

4.3.1.1 Geometrische Korrektur. . . . . . . . ..................................................................... 45

4.3.1.2 Atmosphärische Korrektur . . . . . ............................................................ 46

4.3.2 Bildverarbeitung . . . . . . . . . ....................................................... 47

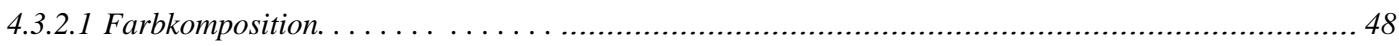

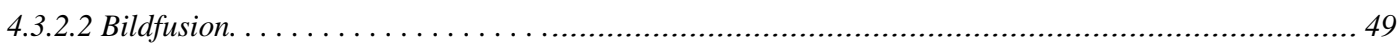

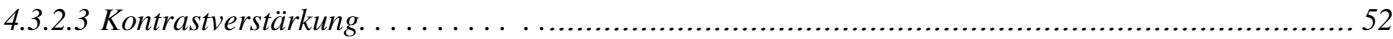

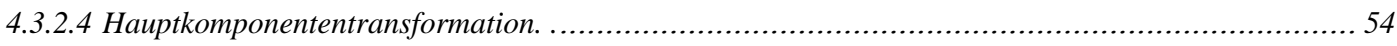

4.3.2.5 Quotientenbilder (Ratiobilder). . . ..................................................................... 56

4.3.2.6 Filtertechniken. . . . . . . . . . . . . .......................................................................... 57

5 DARSTELLUNG DER GEOMORPHOLOGIE DES GEBIETS....................................... 61

5.1 GEOMORPHOMETRISCHE ANALYSE DES ARbEITSGEBIETS. . . ...................................... 63

5.1.1 Ermittlung der Einzugsgebiete und Subeinzugsgebiete ..................................... 65

5.1.2 Die Ermittlung der geomorphometrischen Parameter ........................................ 69

5.1.2.1 Die Höhe (relatives Relief, absolute Höhe) .................................................................. 69

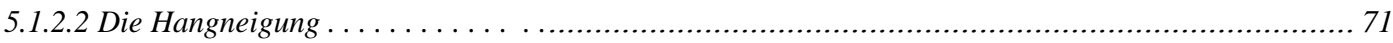

5.1.3 Die Darstellung der morphologischen Haupteinheiten ....................................... 73

5.1.3.1 Täler der Hauptflüsse . . . . . . . . ................................................................ 76

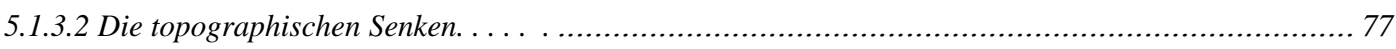

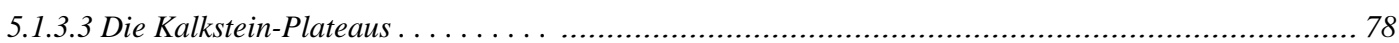

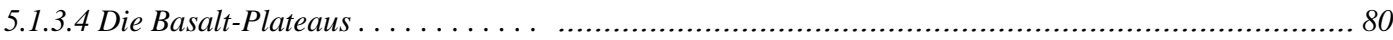

5.1.3.5 Das Bergland . . . . . . . . . . . ............................................................... 80

5.2 ERMITTLUNG DER GEOMORPHOLOGIE MITTELS SATELLITENBILDER ................................. 81

5.2.1 Auswahl der Kanalkombinationen und der Bilder........................................... 82

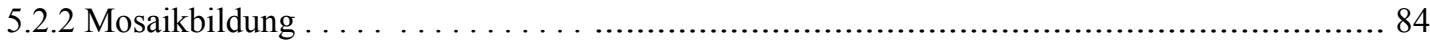

5.2.3 Die geomorphologische Auswertung der Satellitendaten ...................................... 85

6 MORPHOSTRUKTURELLE DARSTELLUNG UND ANALYSE ............................... 97

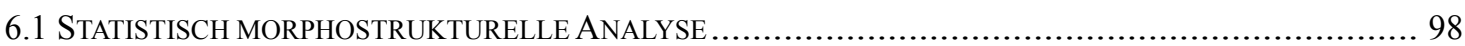

6.2 ViSUELLE ERMitTLUNG DER LINEAREN MERKMALE ................................................. 101

6.2.1 Ermittlung von Lineamenten durch Einsatz des DHMs..................................... 102

6.2.2 Ermittlung von Lineamenten auf den Satellitenbildern .......................................... 105

6.3 Richtungsverteilung der Lineamenten . . ................................................. 107

7 ERSTELLUNG DER HYDROGEOMORPHOLOGISCHEN DATENBANK.......................... 113

7.1 ERMitTLUNG UND GEWICHTUNG DER THEMATISCHEN EBENEN .................................. 116

7.1.1 Thematische Ebene der Böden . . . ........................................................ 118

7.1.2 Thematische Ebene der Landoberflächenformen .............................................. 120

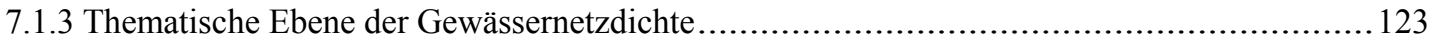

7.1.4 Thematische Ebene der Lineamente............................................................. 124 
7.1.5 Thematische Ebene der Lithologie 125

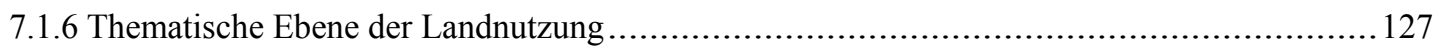

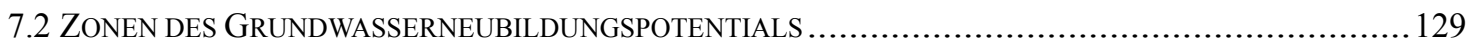

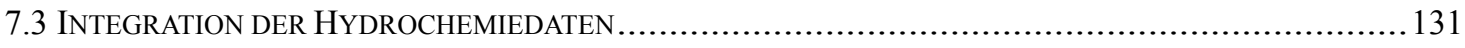

7.3.1 Probenahme und Auswahl der Probenahmebereiche............................................. 131

7.3.2 Klassifikation und Hydrochemie des Grundwassers ........................................ 133

7.3.2.1 Hydrochemie des Teileinzugsgebiets A (Palmyra-Ketten)..............................................138

7.3.2.2 Hydrochemie des Teileinzugsgebiets B (Basaltregion) ................................................141

7.3.3 Beobachtung der hydrochemischen Änderung im Winter ..................................... 142

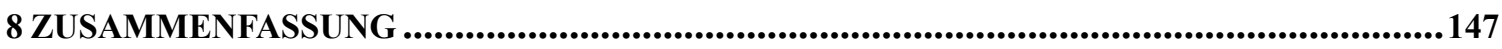

8.1 Die ResUltate .................................................................................. 147

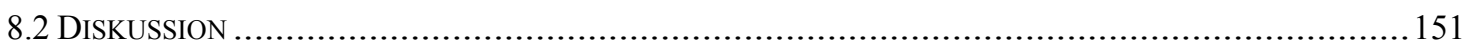

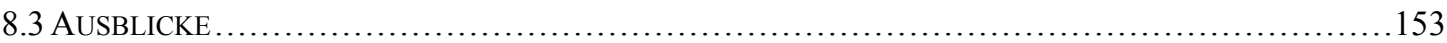

LITERATURVERZEICHNIS

DANKSAGUNG

LEBENSLAUF 


\section{Abbildungsverzeichnis}

Abb. 2-1: Schematische Darstellung des Untersuchungsgebiets

Abb. 2-2: Die tektonische Karte von Syrien (Cornell, 2001) ......................................... 6

Abb. 2-3: Paläogeographische und -tektonische Darstellung (BREW et al., 2001)...................... 10

Abb. 2-4: Die Bildung des hydrografischen Netzes vom Orontes (VAUMAS, 1957): ...................... 13

Abb. 2-5: Die jährlichen Niederschläge in mm (Meteorologie-Abteilung, Damaskus) .................. 18

Abb. 2-6: Klimadiagramme von Aleppo \& Palmyra (geändert nach RöSNER, 1995) ...................... 18

Abb. 2-7: Vereinzelte Bäume von Pistascia atlantica in den Wadis innerhalb J. Bilass .................... 20

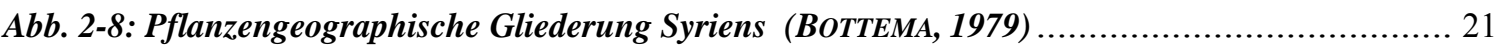

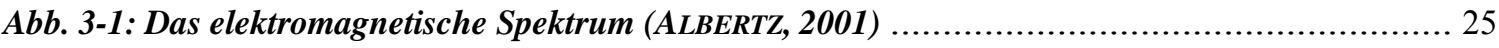

Abb. 3-2: Spektralbereichen der ASTER und ETM+ Daten (PCIGEOMATICS, 16.11.2007).............. 28

Abb. 3-3: Schematischer Aufbau eines Interferometrie-Prozessors (DLR, 02.12.2007) ................ 31

Abb. 3-4: Inhalt der SRTM digitalen Höhendaten (MAIRE \& DATCU, 2004) .............................. 32

Abb. 3-5: Verhältnis zwischen den geoökologischen Elementen und den Elementen der Photointerpretation (GUPTA, 2003).

Abb. 4-1: Ermittlung der Lithologie in Landsatbilder (Rechts) im Vergleich zu geologischen Karten

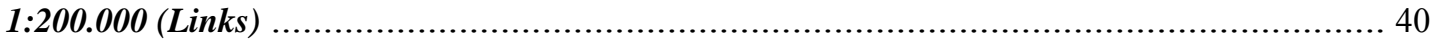

Abb. 4-2: SRTM Rohdaten (Links) und die Daten nach Interpolation (Rechts). ........................ 41

Abb. 4-3: Das geschummerte Reliefmodel der Rohdaten (Links) und nach der Lee-Filterung (Rechts) 42

Abb. 4-4: Fehler der Fließrichtungsdefinition in den Rohdaten 43

Abb. 4-5: Geometrische Ausgabe-Matrix überlagert von Pixeln in unkorrigierter Lage (LILLESAND \&

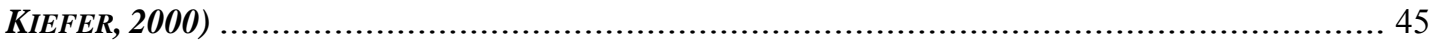

Abb. 4-6: Relative Reaktion der Photopigmente des menschlichen Auges .............................. 48

Abb. 4-7: Fusionsmethoden am Beispiel von ETM+ 741 (von Links HSV, PC, CN) ................... 50

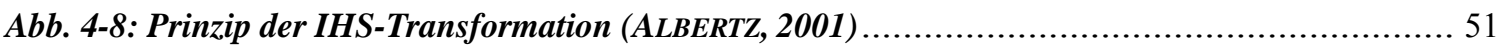

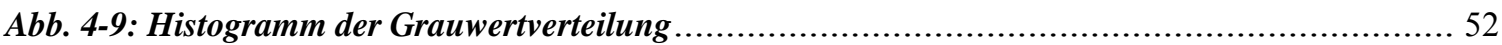

Abb. 4-10: Landsat (741) ohne Erstreckung (Links) und nach der linear (2\%) Erstreckung (Rechts) .. 53

Abb. 4-11: Landsat 731 (Basaltgebiet) links vor der Anwendung des Root-Verfahrens \& rechts nach der

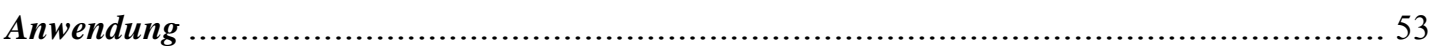

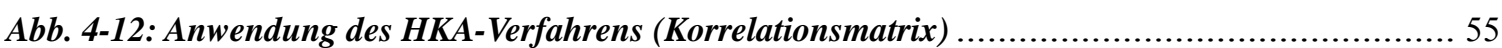

Abb. 4-13: Hervorhebung der linearen Morphostrukturen mittels Richtungsfiltern ..................... 58

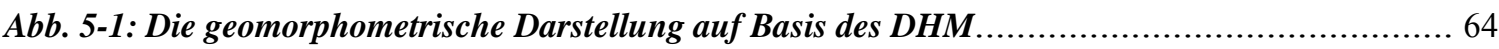

Abb. 5-2: Gliederung des Untersuchunggebiets in Teileinzugsgebieten .............................. 66

Abb. 5-3: Profil Missyaf (oben), Al Sàan (Mitte) und Foto des DSFS-Hangs (Ausblick nach Osten) ... 68 
Abb. 5-4: Das Profil Khan Shaykhun (oben), Khanasser (Mitte) und Al-Bab (unten).... 68

Abb. 5-5: Auf Basis der absoluten Höhe klassifiziertes DHM (links) und Statistische Auswertungen (rechts)

Abb. 5-6: Karte der Hangneigung und Häufigkeitsverteilung 72

Abb. 5-7: Darstellung der Geomorphologie mittels der Höhenlinien (Basis ist DHM) .................... 73

Abb. 5-8: Überlagerung von Landsat ETM+ (741) und DHM (DSFS) Ausblick nach Osten ............ 74

Abb. 5-9: Segmentierung der morphologischen Haupteinheiten (graue Polygone) ....................... 75

Abb. 5-10: Topographische Profile durch den Orontes (Pfeile markieren die Umbiegung) .............. 76

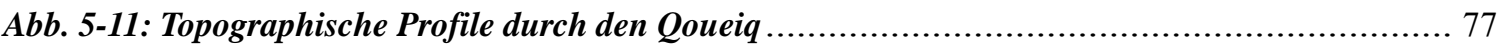

Abb. 5-12: Missyaf-Plateau (Überlagerung der Kombination 741 über das DHM) .................... 78

Abb. 5-13: Abdachung des Zawiye nach Osten (Ausblick nach Westen) ................................ 78

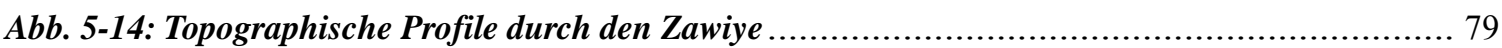

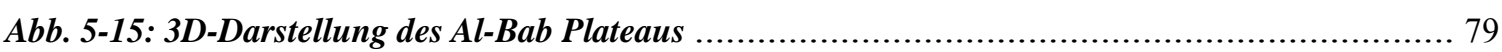

Abb. 5-16: Übergang von der Basaltstufe und dem liegenden weißen Mergel ............................ 80

Abb. 5-17: Die RGB-Kombinationen mit dem höchsten OIF der Szene ETM 173036 (Links 753, Mitte

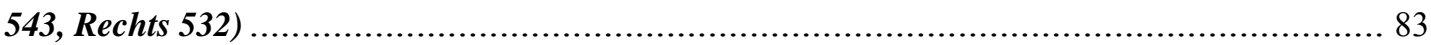

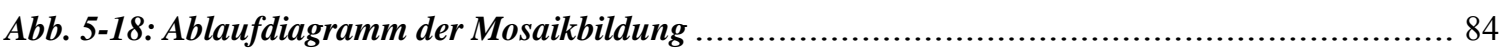

Abb. 5-19: Versalzte Flächen, oben Ratio 3/1, 5/7 und 3/5 \& unten TM 176 (RGB) ..................... 86

Abb. 5-20: Ratiokombination 3/5, 3/1 und 5/7 (RGB). Die Schwemmhalden (Pfeile) und aktiven

Erosionsrinnen sind blau dargestellt.

Abb. 5-21: Schwemmfächerverbreitung im Südost des Untersuchungsgebiet Ratiokanalkombination 3/1, 5/7 und $3 / 5$ (oben) verglichen mit .

Abb. 5-22: Deutlicher Kontrast zwischen dem Basalt und Kalkstein Landsat (741) ....................... 89

Abb. 5-23: Oben (links Wadi innerhalb von dem Basalt. rechts Basalt-Pavement). ....................... 90

Abb. 5-24: Geomorphologische Kartierung am Beispiel vom Hama-Basalt und Landsat 653 (RGB) ... 90

Abb. 5-25: Poljen und Uvalas (Pfeilen) W-Aleppo (oben ETM+ 541, unten TM 546) .................. 92

Abb. 5-26: Kantenverschärfung durch die Anwendung von dem Sobel Filter ........................... 92

Abb. 5-27: HSV-Fusion des ETM+ (741+8) \& die geomorphologische Auswertung ...................... 93

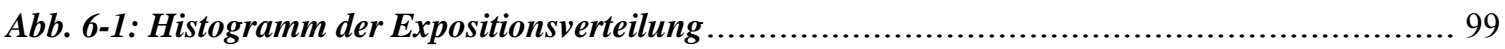

Abb. 6-2: Arithmetische Ausdrücke der verwendeten Filter. ............................................. 100

Abb. 6-3: Exposition verschnitten mit Hangneigung (Basis ist das geschumm. Modell) ................. 100

Abb. 6-4: Einfluss der Azimutänderung auf dem geschummerten Reliefmodel ......................... 103

Abb. 6-5: Geschummertes Reliefmodell (Azimut 135\%), Verwerfungen (Rot) und lineare Phänomenen (Blau) 
Abb. 6-6: Das erzeugte Entwässerungsnetz (Pfeile zeigen einige lineare Merkmale) 104

Abb. 6-7: Verbesserung der visuellen Interpretation mittels PCA-Verfahren; Links Landsat 542 (RGB) und rechts PC4, PC3 und PC2 (RGB)

Abb. 6-8: Vergleich zwischen der Kanalkombination 542 und der Ratiobildkombination 7/5, 5/4 und 3/1

Abb. 6-9: Die Karte der ausgewerteten Lineamente kombiniert mit dem geschummerten Reliefmodel

(Rosendarstellung oben rechts für alle Lineamente)

Abb. 6-10: Die Rosendarstellung der Lineamente aus Bereichen des DSFS, NE-Aleppo-Gebiets und der Palmyra-Ketten .

Abb. 6-11: Rhegmatische Orientierung der orographischen Züge und des Entwässerungsnetzes

(WOLFART, 1967)

Abb. 6-12: Seitenverschiebungsmodell NW-Syrien (CHAIMOV et al., 1990). 110

Abb. 7-1: Ablaufdiagramm der hydrogeomorphologischen Darstellung....

Abb. 7-2: Thematische Karten der gewichteten Kategorien.

Abb. 7-3: Ausschnitt der Hydrogeologiekarte mit den Parametern .

Abb. 7-4: Von Kalkkrusten überdeckte fluviale Ablagerung (links) Kalkkrustenbeseitigung (rechts) .. 118

Abb. 7-5 Böden im Zentrum des Gebiets zwei nach Regenereignis .

Abb. 7-6: Glacisterrassen am Rand der Palmyra-Ketten (Blickrichtung nach SE)

Abb. 7-7: Beispiele für verschiedene Wadis aus dem betrachteten Gebiet.

Abb. 7-8: Erschaffung mehrerer Pufferzonen über Lineamenten von Typ A

Abb. 7-9: Zwei Tage nach dem Regenereignis bleibt das Wasser auf dem Basalt stehen

Abb. 7-10: Porosität, Porengröße und theoretische hydraulische Leitfähigkeit (m/Tag) mehrerer Karbonatgesteine (BRAHANA et al., 1988)

Abb. 7-11: Landsat 741 (RGB) Subszene (links), NDVI Bild (rechts).

Abb. 7-12: Karte des Grundwasserneubildungspotentials

Abb. 7-13: Dreidimensionale Darstellung der Karte des Grundwasserneubildungspotential und der Geologie.

Abb. 7-14: Karte des oberen Grundwasserleiters

Abb. 7-15: Teileinzugsgebiete A \& B und die Wasserabflussrichtung (Pfeile).....

Abb. 7-16: Beziehung zwischen der Leitfähigkeit \& dem TDS des Grundwassers .

Abb. 7-17: Piperdiagramm (links) und die Karte der Probenahmepositionen (rechts)

Abb. 7-18: Das Teileinzugsgebiet $A$

Abb. 7-19: Das Konglomerat des Neogen (links) und Kalkstein der Kreide (rechts)

Abb. 7-20: Das Schöllerdiagramm (links) und Probenpositionen (Basis ist Grundwasserleiterkarte(rechts) 
Abb. 7-21: Schöllerdiagramm der Brunnen 28, 30, 31 und SP9 (links) sowie deren geographischen Positionen (rechts) 140

Abb. 7-22: Das Teileinzugsgebiet B. 141

Abb. 7-23: Schöllerdiagramm einiger Paläogen-Brunnen (schwarz) und Kreide-Brunnen (rot), geographische Positionen (rechts) 142

Abb. 7-24: Piper-Diagramm der Proben vor Regenereignis (Nr.) und danach (Nr. R) 144 


\section{Tabellenverzeichnis}

Tab. 2-1: Stratigraphische Formationen und Grundwasserleiter Nordwest-Syriens .................... 16

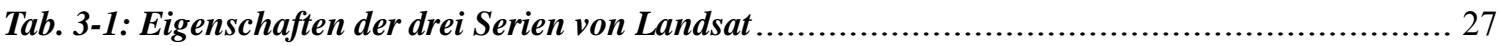

Tab. 3-2: Landsat ETM+ Bandanwendungen (nach KAPPAS, 1994 \& JoNES, 1987)...................... 27

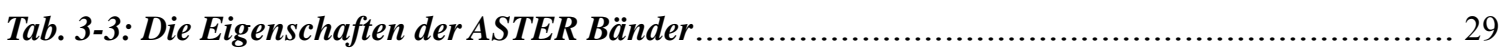

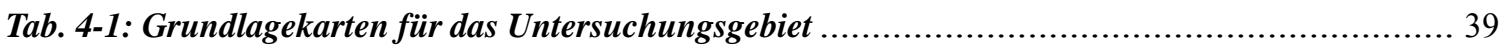

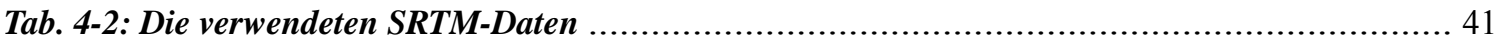

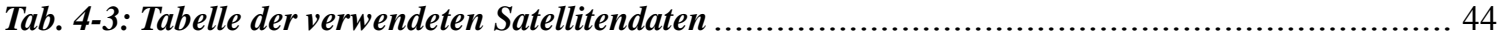

Tab. 5-1: Die Methoden der geomorphologischen Untersuchung (DEMEK et al., 1982).................. 61

Tab. 5-2: Die geomorphologische Klassifikation auf Basis der absoluten Höhe........................ 70

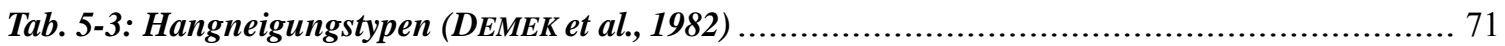

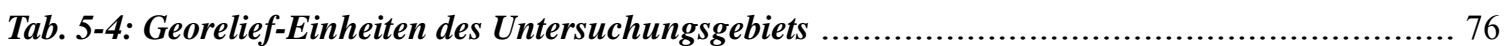

Tab. 5-5: OIF-Verteilung für RGB-Kombinationen der verwendeten Landsatdaten ..................... 82

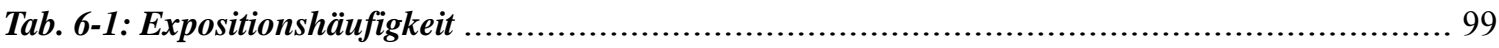

Tab. 7-1: Inhalt der hydro-geomorphologischen Datenbank ...................................... 113

Tab. 7-2: Sechs thematische Ebenen der Datenbank mit ihren Gewichten............................... 115

Tab. 7-3: Gewichtung der verwendeten Daten für Ermittlung der Bodenebene ........................... 119

Tab. 7-4: Gewichtung der Lineament-Pufferzonen ................................................. 125

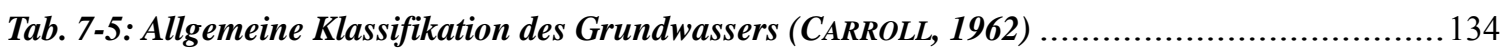

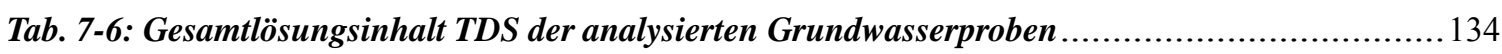

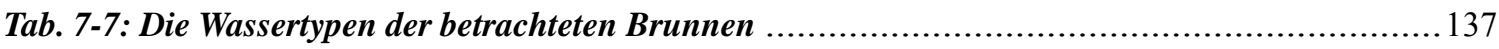

Tab. 7-8: Ionenkonzentrationen des Regenwassers in Homs \& Palmyra (Kattan, 1997)................. 143

Tab. 7-9: Chemischen Zusammensetzung der Brunnen vor und nach dem Regen ....................... 143 


\section{Abkürzungsverzeichnis}

ASI

ASTER

DEM

DHM

DLR

DN

DSFS

DTM

EDV

ETM

FCC

GIS

GPS

HKA

IHS

LIDAR

MSS

NASA

NDVI

NIMA

NIR

$\mathrm{NN}$

OIF

PCA

RADAR

RGB

SPOT

SRTM

SWIR

Tab.

TIR

UTM

VNIR

VIS

Digital Elevation Model

Digitales Höhen Modell

Digital Number

Dead Sea Fault System

Digital Terrain Model

Falce Color Composite

Intensity Hue Saturation

MultiSpectral Scanner

Nahes Infrarot

Normal Null

Optimum Index Factor

Short Wave Infrarot

Tabelle

Thermales Infrarot

Very Near Infra Red
Agenzia Spaziale Italiana

Advanced Spaceborne Thermal Emission and Reflection Radiometer

Deutsche Forschungsanschtalt für Luft- und Raumfahrt

Elektronische Datenverarbeitung

Enhanced Thematic Mapper

Geographisches Informationssystem

Global Positioning System

HauptKomponentenAnalyse

Light Detecting And Ranging

National Aeronautics and Space Agency

Normalized Difference Vegetation Index

National Imagery and Mapping Agency

Principal Component Analysis

Radio Detecting And Ranging

Red Green Blue (Rot Grün Blau)

Système Probatoire d'Observation de la Terre

Shuttle Radar Topography Mission

Universal Transverse Mercator

Visible (sichtbarer Wellenlängenbereich) 


\section{Vorwort}

In den letzten 30 Jahren hat die Elektronische Datenverarbeitung (EDV) Einzug in die Geowissenschaften gefunden und führte zu großen Fortschritten in den angewandten Geowissenschaften. In der angewandten Geomorphologie führte dies zu wesentlichen Weiterentwicklungen (z.B. Anwendung digitaler Geländemodelle), insbesondere auch dadurch, dass diese Wissenschaft sehr eng mit verschiedenen Disziplinen wie Geologie, Fernerkundung, physische Geographie, Hydrologie, Hydrogeologie etc. zusammenarbeitet und durch die EDV-Entwicklung (insb. das geographische Informationssystem) nun unterschiedliche Informationsebenen zusammengebracht werden konnten. Parallel dazu entwickelte sich die geomorphologische Darstellung bzw. Kartierung weiter.

Hierbei erfuhren die geomorphologischen Beobachtungen mit der zunehmenden Entwicklung der Satellitenfernerkundung große Fortschritte. Die Satellitendaten stellen eine hervorragende Möglichkeit zur Verfügung, die geomorphologischen Merkmale und Prozesse in großen Bereichen der Landoberfläche darzustellen. Andererseits bieten die heute vorhandenen Fernerkundungssysteme die Möglichkeit, den Anschluss an den Detailreichtum und Informationsgehalt von Luftbildern zu erreichen. Der Mangel an Höheninformationen kann heute auch überwunden werden, indem man Satellitenbilder mit digitalen Höhenmodellen kombiniert.

Ein weiterer Forschritt wird durch die Anwendung von Geographischen Informationssystemen (GIS) zur Darstellung und Analyse geographischer Features geboten, wobei die Arbeit mit einer Anzahl von mehreren thematischen Ebenen (layer) und verschiedenen Dateien (Texte, Berichte, Fotos, Tabellen, Bilder usw.) in einer geomorphologischen Datenbank unproblematisch geworden ist. In diesem Zusammenhang ermöglicht die geomorphologische Datenbank eine detaillierte Auswertung mehrerer Daten aus unterschiedlichen Quellen, z. B. wird eine Zusammenführung der räumlich und zeitlich variablen, geologischen Fakten und Daten möglich. 
Kapit e l 1

\author{
Ein le it ung
}




\section{$1 \quad$ Einleitung}

\subsection{Zielsetzung}

Die computergestützte Darstellung geomorphologischer/geologischer Informationen mit Hilfe vom geographischen Informationssystem (GIS) bietet einen breiten Anwendungsbereich. Deshalb hat die vorliegende Arbeit zum Ziel, eine ausführliche, GIS-basierte, geomorphologische Darstellung von Teilregionen Syriens zu generieren. Dabei wird die Mehrzweckfunktionalität des GIS, nämlich die Verwendung des GIS-Werkzeugs zum Speichern, Anzeigen und Analysieren (Verschneiden von Datenebenen) für die Lösung verschiedener Fragestellungen eingesetzt. Dementsprechend beinhaltet die Zielsetzung dieser Arbeit folgende Schwerpunkte:

- Zunächst wird eine GIS-gestützte Georeliefdarstellung durchgeführt, um die geomorphologischen Haupteinheiten der natürlichen Landoberfläche in dem Untersuchungsgebiet im nordwestlichen Syrien auf Basis des digitalen Höhenmodells zu klassifizieren und die geomorphologischen Phänomene mittels der Satellitenbilder von Landsat und von dem Advanced Spaceborne Thermal Emission and Reflection Radiometer (ASTER) zu illustrieren. Dabei soll überprüft werden, ob dass digitale Höhenmodell der Shuttle Radar Topography Mission (SRTM) verwendbare Informationen für die geomorphologische Darstellung liefern kann. Weiterhin ist zu überprüfen, ob die ergänzende Auswertung von Satellitendaten eingesetzt werden kann.

- Die Verwendung mehrerer Fernerkundungs- und GIS-Verfahren zur Ableitung einer Karte linearer Morphostrukturen unter Berücksichtigung der strukturellen Eigenschaften des Untersuchungsgebietes, um eine Aussage über den tektonischen Bau des betrachteten Gebiets zu treffen.

- Die Erstellung einer GIS-Datenbank durch die Kombination der ausgewerteten Daten mit weiteren Themen des geoökologischen Systems (Boden, Klima, Lithologie, Landnutzung) und Integration dieser Themen, wodurch die Wirkung der Geomorphologie auf das Grundwasserneubildungspotential und die Grundwasserzusammensetzung übersichtlich dargestellt werden soll. 


\subsection{Methodische Vorgehensweise}

Vor der Bearbeitung des eigentlichen Themas wird das bearbeitete Untersuchungsgebiet inklusive der speziellen Themengebiete (KAPITEL 2) dargestellt. In KAPITEL 3 werden kurz die physikalischen und technischen Grundlagen der Fernerkundung aufgezeigt. Die verwendeten Datengrundlagen und ihre Aufbereitung sowie die benötigten Methoden sind in KAPITEL 4 aufgeführt. Der Kern dieser Arbeit, die morphologische Analyse, insbesondere die geomorphologische Darstellung auf Basis der Fernerkundungsdaten (digitale Höhendaten \& Satellitenbilder), wird in KAPITEL 5 behandelt. Eine morphostrukturelle Analyse wird in KAPITEL 6 durchgeführt. In KAPITEL 7 werden die geomorphologischen Daten mit den Daten der benachbarten Disziplinen in einer hydrogeomorphologischen Datenbank kombiniert. Die verschiedenen bearbeiteten Themen werden in drei Darstellungen repräsentiert:

1. Vektor-Daten für das Darstellen einzelner Objekte.

2. Rasterdaten für das Darstellen von kontinuierlichen Phänomenen.

3. Referenzen (Verzeichnisse) für die geographische Position.

Es wurden zwei Feldkampagnen in dem Gebiet durchgeführt. Dabei wurden die aufgelisteten Ziele verfolgt:

- Einmessen von Passpunkten für die Verifizierung der Georeferenzierung der Fernerkundungsszenen.

- Erfassen der Geomorphologie.

- Erhebung von örtlichen und momentanen Daten für die Verifikation der Auswertungen und der Klassifikationen.

- Proben nehmen.

Die erste Kampagne fand im April 2004 im westlichen Teil des Untersuchungsgebiets statt. Dieser Teil umfasst das Areal des Missyaf-Plateaus und den Bereich des TotenMeer-Störungssystems (DSFS). Die zweite Kampagne fand im September und Oktober 2006 im östlichen und südöstlichen Teil statt. Das Areal erfasst die nördlichen PalmyraKetten und ihr Vorland sowie einen großen Teil der Basaltfelder von Hama. 


\section{Kap it e 12}

Charakterisierung des

Untersuchungs ge bi e tes 


\section{Charakterisierung des Untersuchungsgebietes}

Syrien befindet sich im nordwestlichen Teil der arabischen Halbinsel. Es hat eine Fläche von ungefähr $185.000 \mathrm{Km}^{2}$ und liegt zwischen $32^{\circ}$ und $37^{\circ}$ Nordbreite und zwischen $35^{\circ}$ und $42^{\circ}$ Langbreite. Das Untersuchungsgebiet dehnt sich im nordwestlichen Syrien von $36^{\circ} 20^{\prime}$ bis $38^{\circ} 20^{\prime} \mathrm{E}$, und $34^{\circ} 40^{\prime}$ bis $36^{\circ} 45^{\prime} \mathrm{N}$ (190 km NS * $\left.165 \mathrm{~km} \mathrm{WE}\right)($ Abb. 2-1) aus.

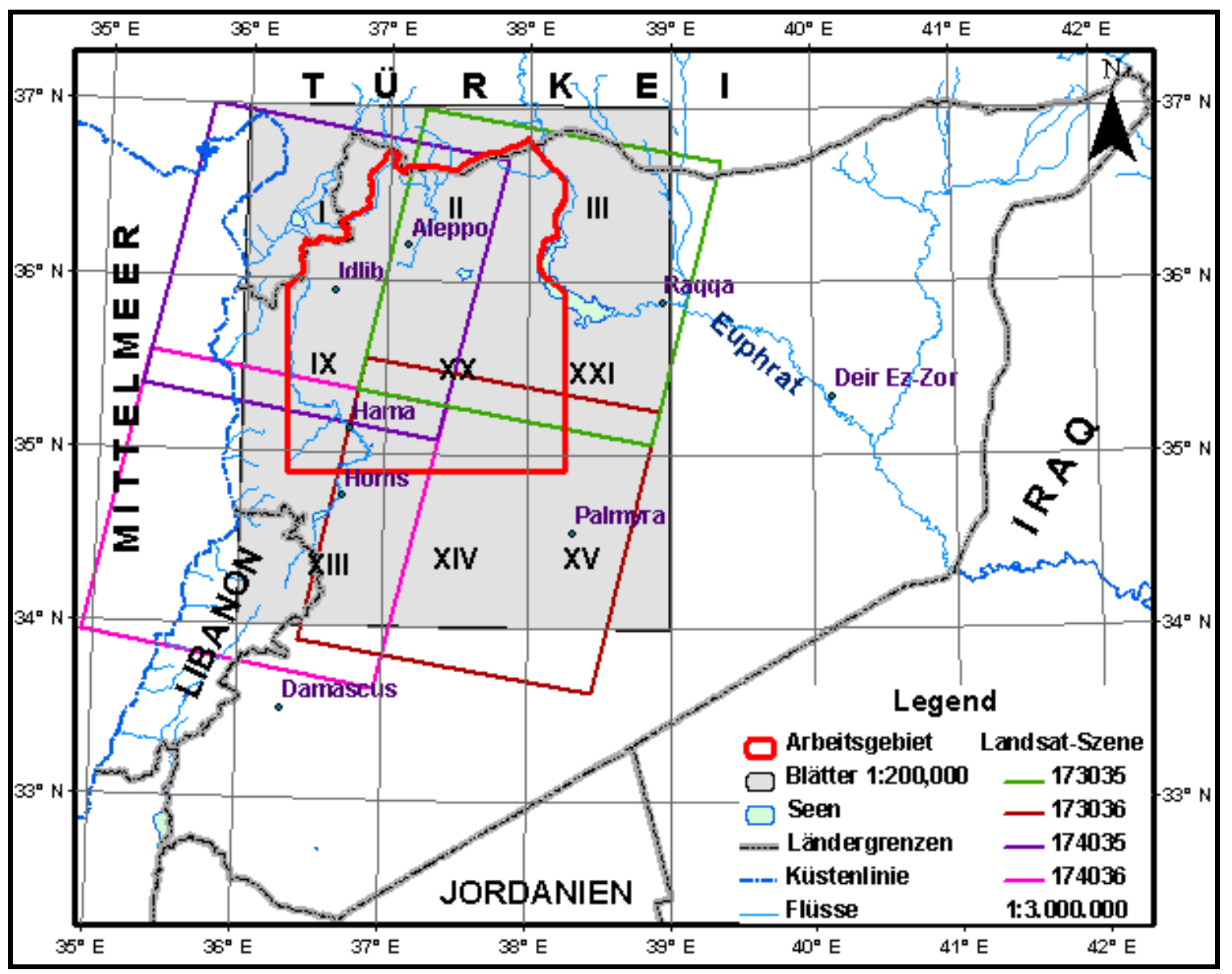

Abb. 2-1: Schematische Darstellung des Untersuchungsgebiets

Im Süden, Westen und Norden ist das Gebiet von gebirgigen Landschaften geprägt. Die Vegetation im südlichen bis südöstlichen Bereich ist spärlich bis nicht vorhanden. Im Norden und Nordwesten sowie im Westen herrscht eine Ackerlandschaft vor. Das Gebirge im Nordwesten ist zum Teil bewaldet und als Teil des Taurus-Gebirges (Türkei) zu bezeichnen. Die Entwässerung des Gebietes erfolgt größtenteils in abflusslose Becken, die fast den gesamten Raum des Zentralgebiets einnehmen. 


\subsection{Geologie}

Syrien befindet sich an der nordwestlichen Ecke des arabischen Blocks (Arabian Plate) und kann in fünf geologische Regionen gegliedert (Abb. 2-2) werden:

1. die südliche Syrien-Plattform.

2. die Palmyra Faltenzone.

3. die Aleppo-Hebung

4. die westlichen Bergketten einschließlich der Graben-Zone des DSFS.

5. der Mesopotamien-Trog (das Euphrat-Verwerfungssystem).

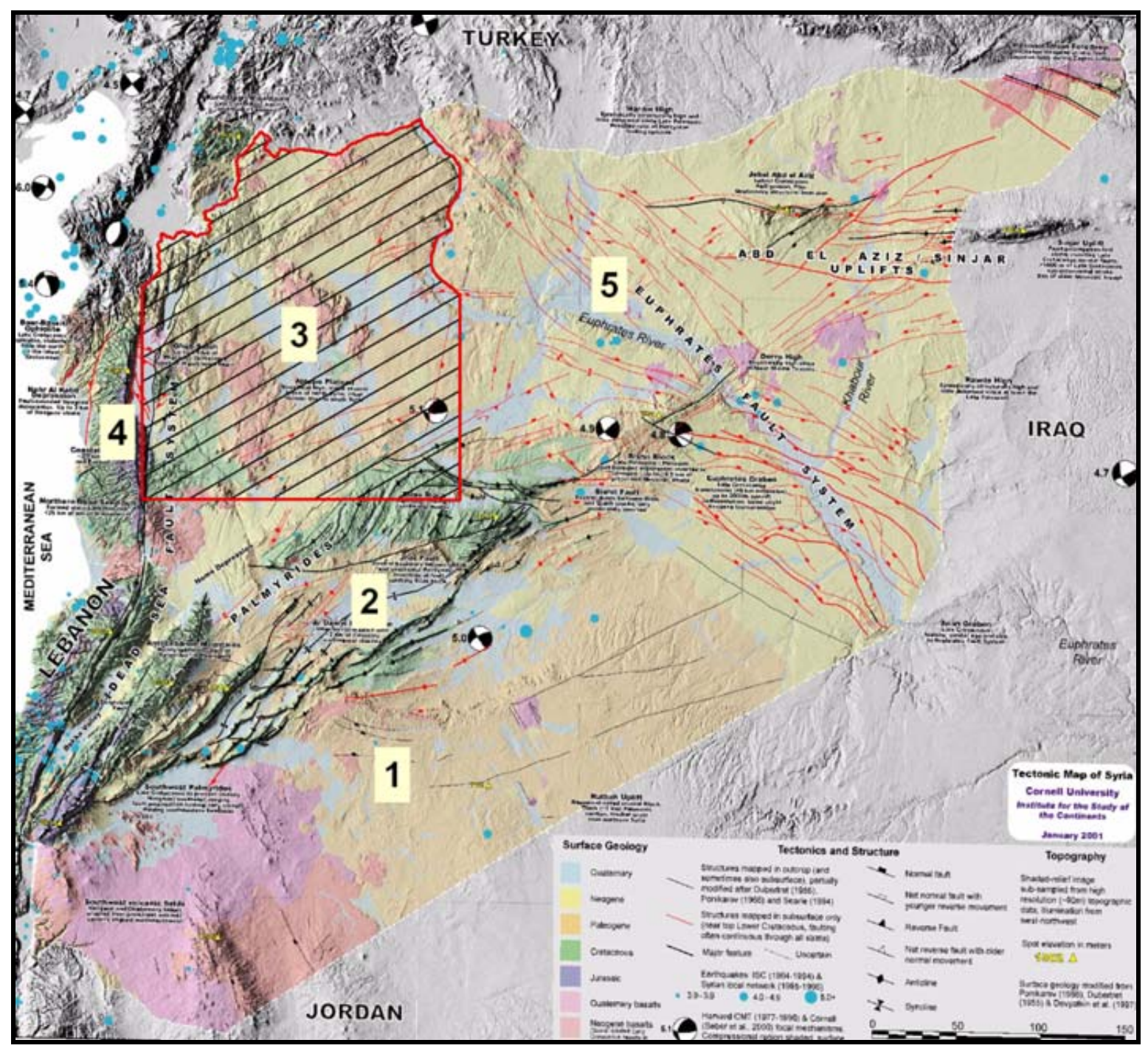

Abb. 2-2: Die tektonische Karte von Syrien (Cornell, 2001) 
Die strukturelle Großgliederung Syriens wie auch des Libanon wird im Wesentlichen von vier tektonischen Richtungen beherrscht (WOLFART, 1967):

i- $\quad$ N-S Richtung (Ostafrikanische Richtung), hauptsächlich in dem meridionalen Graben Al-Ghab von West Syrien und dem Libanon auftretend,

ii- $\quad$ NE-SW Richtung,

iii- NW-SE Richtung,

iv- $\quad$ E-W Richtung.

Die zweite und dritte tektonische Richtung sind praktisch in ganz Syrien verbreitet. Die vierte hingegen ist vor allem im Nordosten Syriens vorhanden (Abb. 2-2). Im Grunde genommen wird der tektonische Großbau Syriens im Wesentlichen von den Strukturelementen der NW- und NE-Richtung beherrscht, deren Bildung in engem Zusammenhang mit den präexistenten Fugen des Transversalverschiebungssystems stehen dürfte. WOLFART (1967) beschrieb, dass selbst im Bereich der meridionalen Graben-Zone im Westen Syriens diese Regel eine gewisse Gültigkeit besitzt. Als Beweis nannte er den ablenkenden Effekt des Transversalverschiebungssystems auf junge Brüche und vor allem auf die Orientierung des libanesischen Gebirgssystems und des Bekaa-Grabens (Abb. 2-2). Für das betrachtete Gebiet sind die ersten drei Richtungen von größerer Bedeutung.

\subsubsection{Geologische Entwicklung des Gebiets}

Das Untersuchungsgebiet beinhaltet das Aleppo-Plateau (Aleppo-Uplift). Im Westen ist das Becken von Al-Ghab bzw. das DSFS als Grenze zu betrachten und im Nordwesten das Aafrin-Becken bzw. die Bitlis Suture (HeMPton, 1985). Im Osten lässt sich das Aleppo-Plateau vom Euphrat-Verwerfungssystem abgrenzen. Im Süden und Südosten überschneiden sich teilweise das Aleppo-Plateau und die Palmyra-Ketten. Das AleppoPlateau war während des Paläozoikums eine von zwei strukturellen Erhebungen in Syrien, Aleppo-Hebung im Nordwesten und Rutbah-Hebung (Rutbah uplift) im Südosten (Abb. 2-2) (BREW et al., 2001).

Im Mesozoikum erfuhr das Gebiet mehrere Transgressionen und Regressionen. Demgemäß herrschten auf der Aleppo-Hebung marine bis lagunäre, gebietsweise auch kontinentale Verhältnisse, unter denen es unter anderem zur Ablagerung von Anhydriten oder so- 
gar von Steinsalz gekommen ist. Am Ende des Mesozoikums (späte Kreidezeit) wurde Syrien von mehreren subsidierenden Bereichen beherrscht. Folglich bildete sich das Aafrin-Becken nordwestlich der Aleppo-Hebung und Ophiolit-Eruptionen traten an dem nordwestlichen Rand auf.

Während des Paläogens (Känozoikum) erfuhr das Gebiet eine aktive Tektonik. Einerseits wurden Subsidenz-Prozesse sowohl südlich des Aleppo-Plateaus (im Bereich der Palmyra-Ketten) als auch östlich (Euphrat-Graben) dokumentiert. Andererseits erhob sich das Küsten-Gebirge im Westen und das Kurd Dagh Gebirge im Nordwesten. Fazies bezogen, gehen überall in Syrien unmerklich die blaugrauen Mergel und hellen, weißen, kreidigen Mergelkalke der Oberkreide in die völlig gleichförmigen Schichten des frühen Paläogens über. Diese Schichtenfolge ist in ganz Syrien bzw. innerhalb des Aleppo-Plateaus horizontal homogen.

Das frühe Eozän zeigt eine seichtere Fazies als die Fazies im Paleozän und in der Oberkreide. Diese Fazies ist allgemein karbonat- und kieselhaltig. Im mittleren Eozän nahm die Verbreitung der Paläogen-Sedimentationen im Aleppo-Plateau allmählich zu. Mächtige Sedimente wurden im Nordwesten abgelagert. Währenddessen wurde der Zawiye, der vorher - mit dem Küsten-Gebirge erhoben - exponiert war, überflutet. Überall in Syrien wurden pelagische, kreidige Mergelkalke und Mergel abgelagert. Doch davon weichten die überfluteten, gehobenen Bereiche (Zawiye) ab. Hier und auch im Südosten wurden Konglomerate und Grünsande in geringer Mächtigkeit und Bankkalke abgelagert. Die merglig-kreidige Sedimentation des Eozäns folgt im oberen Paläogen von sandigen Nummulitenkalkstein und Sandstein. So hat am Ende des Paläogen nach den tektonischen Erhebungen möglicherweise keine marine Sedimentation mehr stattgefunden. Hingegen traten in mehreren Zeiten während des Neogen in den verschiedenen Teilen des Gebiets (wie dem Aafrin Becken) marine Bedingungen auf, welche auf die Wirkung der tektonischen Hebung zurückzuführen ist.

Basaltschichten, besonders des mittleren Miozän, zeigen vulkanische Tätigkeit an, zu denen gehört das vulkanische Vorkommen südlich des Aleppo-Plateaus. Wie die anderen Mittelmeergebiete zeichnet sich die Messinzeit (Eng. Messinian) durch die Absetzung von Evaporiten aus. Die stratigraphische Reihenfolge des Pliozäns zeigt eine Weiterentwicklung in Richtung der kontinentalen Absetzung mit Basaltvulkanismus. 
Nicht nur das Aleppo-Plateau sondern fast ganz Syrien wurde zu Beginn des Pliozäns durch die exogenen Kräfte von Verwitterung, Abtragung bzw. Aufschüttung geprägt. Dieser Einebnungsvorgang formte ein Flachrelief, das zum Ausgangspunkt für die heutigen Großformen wurde. Junge Krustenbewegungen während des Pliozäns und Quartärs führten im Westen zu lokal begrenzten Hebungen, Senkungen und Verbiegungen. Infolgedessen wurde der Grabenbruch von Al-Ghab westlich des Aleppo-Plateaus gebildet. Gleichzeitig kam es in dem Gebiet zu pliozänem bis quartärem Vulkanismus. Im Quartär hatten sich auch die europäischen Eiszeiten und Zwischeneiszeiten in Syrien als Pluvial- bzw. Trockenperioden bemerkbar gemacht. Darauf deuten die limnischen PleistozänAblagerungen der großen Gräben v. a. dem Al-Ghab.

\subsubsection{Die paläogeographische Entwicklung der Landschaft}

Die paläogeographische Entwicklung während der oberen Kreide und während des unteren Tertiärs hatte schließlich im Eozän, gebietsweise auch erst im unteren Oligozän, zu einer umfassenden Regression geführt. Darauf deuten die frühen Eozän Konglomerate und Grünsande im Zawiye und in den nördlichen Palmyra-Ketten. Das kontinentale Verhältnis sollte nach WOLFART (1967) schon in Syrien gegen Ende des unteren Tertiärs bei einem ausgeglichenen Relief fast überall herrschen. Dieser gerichtete Entwicklungstrend wurde mit Beginn des jungen Tertiärs angehalten.

Eine moderne Darstellung für die Sedimentationslage und das tektonische Abbild im oberen Eozän zeigt an, dass eine Flachwasserfazies herrschte. Gemäß dieser Darstellung lag im mittleren Miozän im Vergleich zum oberen Eozän eine noch ausgeprägte Flachwasserfazies vor (Abb. 2-3), so dass ein Salinarbecken östlich des Aleppo-Plateaus gebildet wurde. Nach WOLFART (1967) dehnte sich das Salinarbecken vermutlich nach Westen bis östlich von Homs aus und zwar entlang einer Meeresbucht, die sich als Teil des Euphrat-Beckens im Bereich der tektonischen Palmyra-Faltenzone erstreckte.

Das Miozän zeichnet sich durch den abschließenden Übergang zu den kontinentalen Bedingungen in Syrien (BREW et al., 2001) aus, so dass Nordsyrien während des oberen Teils (Pont) des Miozän definitiv vom Meer auftauchte. Ab diesem Zeitpunkt bildeten sich Berge, wodurch das Relief ausgeprägter wurde (VoÛTE, 1955). WOLFART (1967) deutete auf eine bereits im Miozän ausgebildete, nach Nordosten zum Euphrat-Becken gerichtete Abdachung hin, welche sich durch die Verbreitung der kontinentalen Sedimente, durch die generellen Schüttungsrichtungen und durch die an das paläogeographische 
Bild des Miozäns anknüpfenden Überlagerungen bestätigen lässt. Im obern Miozän (Pont) waren zwei Süßwasserseen vorhanden, einer im Graben von Al-Ghab und der andere in der seichten Depression von Homs (DUBERTRET \& VAUTRIN, 1938; Voûte, 1955). Lakustrische, tonig-mergelige Ablagerungen des Pont kommen im Al-Ghab und bei Homs sowie in der Bekaa-Ebene (südlich von Homs im Libanon) vor. Im Allgemeinen wurde ganz Syrien vermutlich in dem Pont von einer schwach reliefierten Ebene charakterisiert. Diese pontische Einebnungsfläche ist in weiten Tälern Syriens bis heute erhalten und sogar überall, wo sie nicht durch nachfolgende Krustenbewegungen verstellt wurde (VAUMAS, 1961). Auf eine Flächenbildung, die offenbar zwischen Helvet (oberen Miozän) und unterem Pliozän erfolge, deutete RöSNER (1995) in einer Studie über die Entwicklung der Landschaft nördlich Aleppo hin.

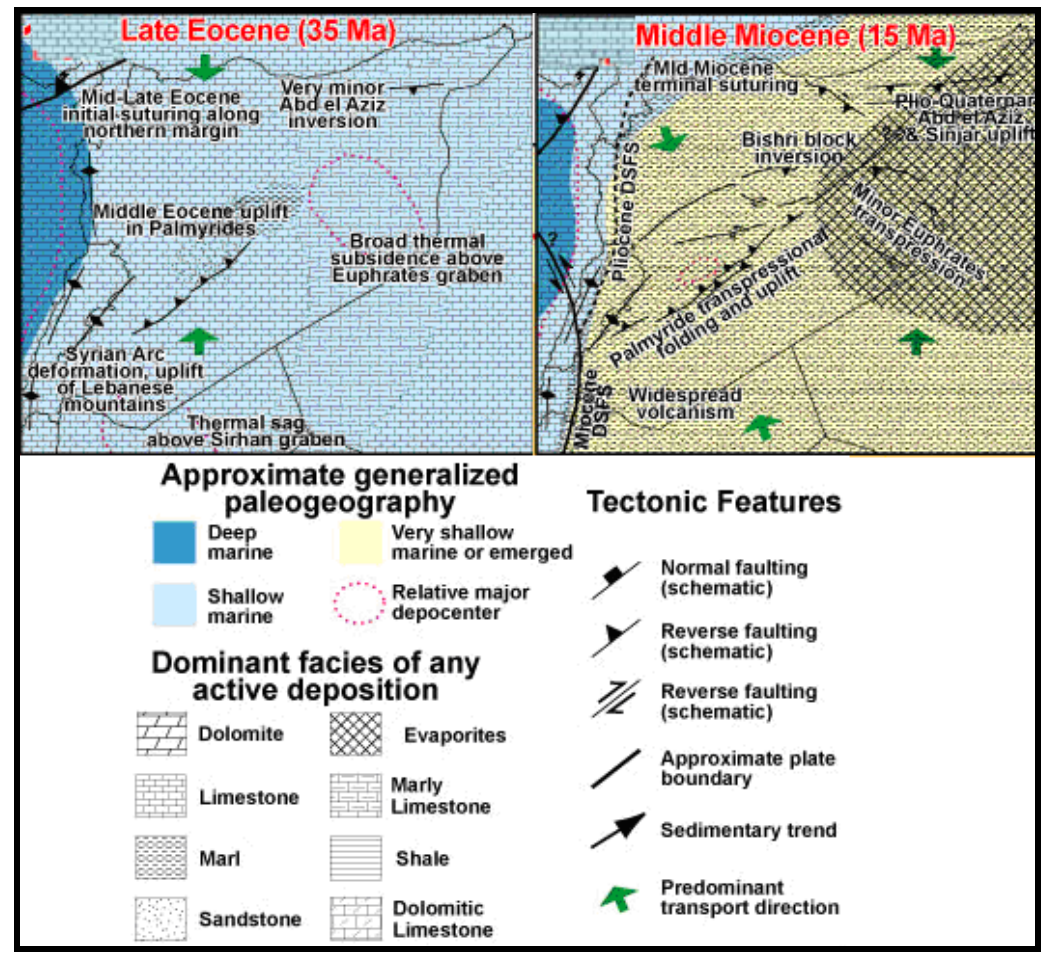

Abb. 2-3: Paläogeographische und -tektonische Darstellung (BREW et al., 2001)

Mit Beginn des Pliozäns und im Pliozän wurde durch kräftige Belebung des Reliefs im Westen eine starke Erosion ausgelöst und die Ausdehnung der aus dem oberen Miozän stammenden Seen eingeengt. Im Pliozän entwickelte sich ein ausgedehntes recht einschneidendes Entwässerungsnetz, das den Abtragungsschutt der syrisch-libanesischen Gebirge vom Westen nach Osten im Bereich der Palmyra-Ketten verfrachtet hat, worauf die Neogen-Konglomerate zurückzuführen sind (WOLFART, 1967). 
Die paläogeographischen Verhältnisse des Pliozäns dauerten im kontinentalen Bereich bis in das Altpleistozän an. Es ist überwiegend von Eintiefung und Talbildung gekennzeichnet. Die Täler wurden sogar in Gebieten mit relativ niedrigem Niederschlag ausgeräumt. Gegenteilig verlief die Entwicklung im mittleren Pleistozän. Eine Einebnung der Landoberfläche fand statt, wobei die übertieften Täler sich z. T. mit Abtragungsschutt füllten. Die Mächtigkeit der Talfüllungen könnte beträchtlich größer gewesen sein als heute (VAN LIERE, 1960/61). Im jungen Pleistozän wurden die Täler unter Einfluss höherer Niederschlagereignisse erneut vertieft und die größten Teile der mittleren Pleistozän Talfüllungen wieder ausgeräumt. Die meisten Wadis der syrischen trockenen Gebiete sind im Pleistozän und Holozän angelegt und enden in abflusslosen Becken. Es gibt auch die Vermutung, dass manche Wadisysteme im Südwesten Syriens noch älter sind und deren Anlage schon im Oligozän erfolgte (BöSCH, 1949).

Der große See vom Al-Ghab wurde während des Pliozäns durch das große Verwerfungssystem (DSFS) in den Graben von Al-Ghab und das Becken von Acharneh (Nordwestlich von Hama) geteilt. Zwei meridional verlaufende Verwerfungen haben Al-Ghab in seiner heutigen Form geprägt. Im Acharneh-Becken traten lokal mehrere Verwerfungen auf, die nicht exakt meridional verlaufen. Die Strukturen und Sedimente, die in dem AcharnehBecken gefunden wurden, scheinen anzuzeigen, dass die tektonischen Bewegungen in dessen Umgebung vor und nach dem oberen Miozän das Acharneh-Becken beeinflussten. Vermutlich gab es während des Pliozäns und Quartärs keine weiteren wichtigen Bewegungen. Das Acharneh-Becken ist als ein Pull-Apart Becken festgestellt, das älter ist als das Al-Ghab Pull-apart Becken (VOÛTE, 1955). Nordöstlich dieses Bereichs waren innerhalb des Aleppo-Plateaus während des Quartärs mehrere seichte Becken bekannt. Nordöstlich von Aleppo befand sich das Becken von Al-Bab, in dem sich die nur 3-4 m mächtigen Schotterfächer mit den lakustrischen Gips-Mergeln im Endseegebiet von AlJabboul verzahnen.

Nördlich von Aleppo war ebenfalls ein kleines Becken (Mareaa) vorhanden. In diesem Becken wurden 40-60 m mächtige rote Tone erbohrt, die möglicherweise als Zusammengeschwemmte Verwitterungsreste von Basalten zu deuten sind (WIRTH, 1971). Südlich von Aleppo gab es die Senke von Al-Match, in der dagegen keine nennenswerten fluviatil-limnischen Schichten vorhanden sind. Südlich von Al-Match ist auch die seichte AlKharaitch Depression angesiedelt. Die genannten Binnenbecken dürften erst im Laufe des Quartärs entstanden sein (WOLFART, 1967). 


\subsubsection{Die Paläogeographie des Euphrat und Orontes}

Die Anlage des Euphrat, der einer tektonischen Leitlinie, dem Mesopotamischen Trog, folgt, reicht bis ins Miozän zurück. In dieser Zeit herrschten zwischen dem ZagrosGebirge im Osten und dem Aleppo-Plateau im Westen noch lagunäre Verhältnisse. Während des Pliozäns hat der Euphrat seine Fracht auf eine Breite von ca. $100 \mathrm{~km}$ abgesetzt, die das gleiche Alter wie die ältesten Schuttfächer im Westen Syriens haben (WOLFART, 1967).

Zum Euphrat entwässerten möglicherweise im Altpleistozän das Einzugsgebiet des heutigen Orontes und das Einzugsgebiet des Aafrin ebenso wie die heute abflusslosen Becken nördlich der Palmyra-Ketten (VAUMAS, 1957 \& 1961). Entsprechend dieser Auffassung floss ein Ur-Orontes aus der Gegend von Homs nach Nordosten durch das Gebiet der Al-Kharaitch- und Al-Jabboul-Depression zum Euphrat bis zum Ausbruch der Basaltlaven nördlich von Hama und südöstlich der Al-Jabboul. Nach Entstehung der Basaltdecken im oberen Pliozän (Villafranchien) war der Fluss an den Südostrand der Basaltdecke abgedrängt, entwässerte jedoch noch in etwa gleicher Richtung durch die beiden o. g. Depressionen und durch das Wadi von Khannasser zum Euphrat (Abb. 2-6). Unter dem Einfluss der jungen tektonischen Absenkungen entlang des Verwerfungssystems des Toten Meeres entstanden drei große pleistozäne Binnenseen im Umkreis von Homs, im AlGhab und bei Antakya (Amigk in der Türkei). Erst im jungen Pleistozän dürfte sich der jetzige Lauf des Orontes ausgebildet haben, der die genannten drei Seen anzapfte und ihre Becken zum Mittelmeer hin entwässerte. Infolgedessen ist das Gewässernetz zwischen Euphrat und Orontes in mehrere abflusslose Becken zerfallen.

Die Klärung der flussgeschichtlichen Entwicklung der größten syrischen Flüsse (Euphrat, Orontes) war das Ziel detaillierter Untersuchungen von VAUMAS (1957), VAN LIERE (1960/61) u. a. 


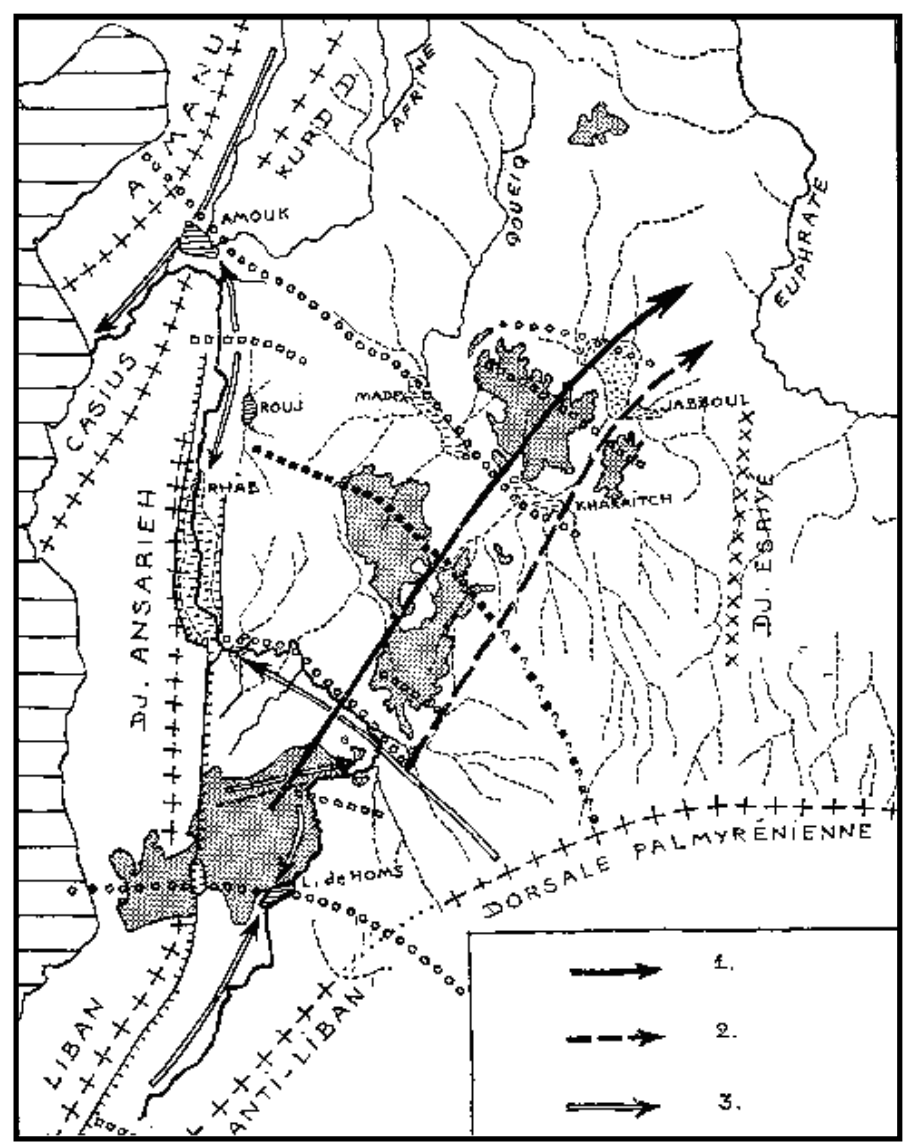

Abb. 2-4: Die Bildung des hydrografischen Netzes vom Orontes (VAUMAS, 1957): 1- der Verlauf von Orontes vor der Basaltintrusion, 2- der Verlauf von Orontes nach der Basaltintrusion, und 3- Entwässerungsrichtungen während der Phase der geschlossenen Becken.

\subsubsection{Lithostratigraphische Einheiten}

Das betrachtete Gebiet liegt zum großen Teil innerhalb des Aleppo-Sedimentbeckens. Das Becken wird durch eine Reihe von verschiedenen hauptsächlich marinen Ablagerungen gefüllt. Anhand der geologischen Karten 1:200.000 (siehe Datengrundalge) können folgende lithostratigraphische Einheiten unterschieden werden:

- Die Oberkreide-Ablagerung besteht aus Kalkstein, Mergel und Dolomit sowie untergeordnet aus Ton und kieselhaltigen Gesteinen. Die Gesteine sind am Gefälle der nördlichen Palmyra-Ketten und im Westen des Gebiets zu finden. Eine 5m mächtige Schicht von Gips liegt an der Basis des Coniacs. Die weit verbreitete aufgeschlossene Ablagerung gehört dem Maastricht an und befindet sich ebenfalls im Zentrum des Gebiets.

- Die Paläogen-Ablagerung setzt sich hauptsächlich aus kreidigen und lehmigen Kalksteinen und untergeordnet aus Mergel zusammen. Diese Ablagerung enthält manchmal dünne Linsen und Schichten von silifiziertem Kalkstein, die im unteren Eozän häufiger als im Mittleren zu finden sind. 
- Die Neogen-Ablagerung wurde hauptsächlich durch die Ablagerungen des unteren Miozäns (Helvet) vertreten. Diese bestehen aus monotonen Schichten des organischen und organisch-detrogenen Kalksteins und zwischengelagerten Basaltsschichten. Der Kalkstein ist grob, von hellgrauer oder grauer Farbe und drastisch gebankt. Die Helvetien Ablagerungen sind im Nordteil des Gebiets weit verbreitet. Zum oberen Miozän gehören die Basaltvorkommen, die ein altes geebnetes Relief überlagern, das aus den Paleozän und Helvetien Ablagerungen besteht. Der Basalt ist stark an seiner Oberfläche geklüftet.

- Die Quartär-Ablagerungen bestehen aus den lakustrischen, alluvialen und proluvialen Ablagerungen und befinden sich in den Depressionen und einigen Tälern. Die proluvialen Ablagerungen werden z. B. am Fuß des Jebel El Hess in einem 1-2 km verbreiteten Gürtel gefunden und werden durch alluviale und lakustrische Ablagerungen zur Mitte der Senken ersetzt. Die proluvialen Ablagerungen bestehen aus schlecht sortierten Schotter des Basalts, des Kalksteins und des Feuersteins in einer gipshaltigen Lehmmatrix. Die lakustrischen Ablagerungen bestehen aus gipshaltigen Lehm und zwischengelagerten Gipsschichten und -linsen.

\subsection{Hydrographie}

Nach WOLFART (1967) lässt sich Syrien hydrographisch in drei Zonen gliedern und zwar West-, Zentral- und Nordost-Syrien, womit das Tafelland nördlich der Palmyra-Ketten zur Zentralzone gehört. Nordöstlich des Gebiets liegt der wichtigste Fluss in Syrien der Euphrat mit seinem großen See. Entlang dem westlichen Rand fließt, einerseits von Süden nach Norden der Orontes innerhalb des Al-Ghab Beckens, andererseits der Aafrin vom Norden in der Türkei nach Südwesten. Die Täler des Aafrin und Orontes weisen westlich der Stadt Aleppo gleichmäßiges Gefälle zum Mittelmeer auf.

Die Basaltdecken bzw. die Ebenen des nordsyrischen Gebietes sind von mehreren breiten Tälern durchgetrennt, die bis zu 100-150m Tiefe aufweisen, und entwässern entweder zum Euphrat oder in abflusslose Depressionen (Sebkhas). Diese sind im Allgemeinen die einzigen natürlichen stehenden Gewässer, die in den syrischen Trockengebieten vorkommen können. Die wichtigste Sebkhas hier sind Al-Jabboul, Al-Match und AlKharaitch. Außer dem Sebkha von Al-Jabboul fallen die anderen Sebkhas im Sommer trocken. 


\subsection{Hydrogeologie}

Der Grundwasserspiegel lag bis vor einigen Jahren, vor der Ausbreitung der intensiven und grundwasserabsenkenden Pumpbewässerung in den Ackerebenen West- und Nordsyriens, meist nur wenige Meter unter der Oberfläche. Bis Mitte des 20. Jahrhunderts konnte man überall in Syrien das Grundwasser durch Brunnen erschließen.

Nach WOLFART (1967) sind Grundwasserkörper in regionaler Verbreitung lediglich im Bereich der Jezireh vorhanden. WOLFART meinte, dass in anderen Gebieten die besten Möglichkeiten zur Grundwassergewinnung sich in den Grundwasser-Sammlersystemen bieten, und zwar vor allem im Vergitterungsbereich lineament-tektonisch bedingter Kluftscharen. Die Vergitterungsbereiche der Lineamente bilden immer Senkungsfelder und wirken daher im Prinzip wie ein Vorfluter.

Hinsichtlich der geringen Niederschläge und der illegalen intensiven Nutzung sinkt der Grundwasserspiegel Jahr für Jahr ab, womit die Wasserversorgung und die Bewässerung des Ackerlands in Frage gestellt werden. Hydrologische Untersuchungen von BURDON (1961) ergaben zum Beispiel für das Gebiet von Salamiyeh (südwestlich des Untersuchungsgebiets), dass vermutlich nur 5\% der über dem Grundwasserspiegel liegenden Flächen bewässert werden dürften (WIRTH, 1971).

Hydrogeologisch in der regionalen Skala spielen der verkarstete Kalkstein und Dolomit der Oberkreide (Cenoman, Turon, Santon und Campan) im Westen von Syrien, Libanon und Jordanien eine sehr wichtige Rolle.

Im Gebiet von Aleppo bilden diese Karbonatgesteine einen tieferen Grundwasserleiter, der durch einen mächtigen Mergel überlagert wird. Über diesem Grundwasserleiter liegt ein weiterer Grundwasserleiter, der aus Kalkstein und mergligen Kalkstein des Paläogens besteht (WAGNER \& GEYH, 2000). Andere vereinzelte Grundwasserleiter befinden sich lokal in mehreren Teilen des Gebiets (Tab. 2-1). 
Tab. 2-1: Stratigraphische Formationen und Grundwasserleiter Nordwest-Syriens

\begin{tabular}{|c|c|c|c|c|c|}
\hline \multicolumn{2}{|c|}{ Stratigraphie } & $\begin{array}{c}\begin{array}{c}\text { Grundwasser- } \\
\text { Leiter }\end{array} \\
\end{array}$ & Lithologie & $\begin{array}{l}\text { Mächtig- } \\
\text { keit (m) }\end{array}$ & $\begin{array}{c}\text { Hydrogeologische } \\
\text { Eigenschaften }\end{array}$ \\
\hline 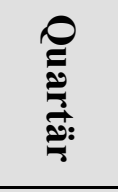 & & \multirow{7}{*}{ Obere } & \multirow[t]{2}{*}{$\begin{array}{l}\text { Lehm, Klasti- } \\
\text { sche Ablage- } \\
\text { rungen \& } \\
\text { Gips }\end{array}$} & \multirow[t]{2}{*}{$10-50$} & \multirow[t]{2}{*}{$\begin{array}{c}\text { Grundwasserleiter } \\
\text { mit geringer } \\
\text { Ergiebigkeit }\end{array}$} \\
\hline \multirow{4}{*}{$\begin{array}{l}Z \\
8 \\
8 \\
00 \\
0 \\
0\end{array}$} & Pliozän & & & & \\
\hline & $\begin{array}{l}\text { Oberes } \\
\text { Miozän }\end{array}$ & & Basalt & $5-30$ & $\begin{array}{c}\text { Lokale } \\
\text { schwebende } \\
\text { Grundwasserleiter }\end{array}$ \\
\hline & Torton & & $\begin{array}{l}\text { Mergel, Kies } \\
\text { und Kalkstein }\end{array}$ & $10-20$ & $\begin{array}{l}\text { Lokale schwebende } \\
\text { Grundwasserleiter }\end{array}$ \\
\hline & Helvet & & $\begin{array}{l}\text { Verkarstete } \\
\text { Kalkstein }\end{array}$ & $5-50$ & Grundwasserleiter \\
\hline \multirow{3}{*}{ 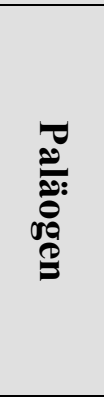 } & Oligozän & & $\begin{array}{c}\text { Mergelige } \\
\text { Kreide, Krei- } \\
\text { de und Kalk- } \\
\text { stein }\end{array}$ & $40-100$ & $\begin{array}{l}\text { Hauptgrund- } \\
\text { Wasserleiter }\end{array}$ \\
\hline & Eozän & & $\begin{array}{l}\text { Nummuliti- } \\
\text { sche verkars- } \\
\text { tete Kalkstein }\end{array}$ & $0-100$ & $\begin{array}{l}\text { Diskontinuierlicher } \\
\text { Grundwasserleiter }\end{array}$ \\
\hline & Paleozän & & \multirow{2}{*}{$\begin{array}{l}\text { Mergel, Krei- } \\
\text { de und Feuer- } \\
\text { stein }\end{array}$} & \multirow[b]{2}{*}{$0->460$} & \multirow[b]{2}{*}{ Aquiclude } \\
\hline \multirow{2}{*}{ 중 $\frac{0}{0}$} & $\frac{\text { Maastricht }}{\text { Campan }}$ & & & & \\
\hline & $\begin{array}{l}\text { Cenoman } \\
\text {-Turon }\end{array}$ & Tiefe & $\begin{array}{c}\text { Verkarstete } \\
\text { Dolomite und } \\
\text { Kalkstein } \\
\end{array}$ & $250-350$ & $\begin{array}{l}\text { Hauptgrundwasser- } \\
\text { leiter }\end{array}$ \\
\hline $\begin{array}{l}\bar{\lambda} \\
\text { 들. } \\
\frac{0}{0} \\
\frac{0}{0}\end{array}$ & $\mathrm{Alb}-\mathrm{Abt}$ & & Mergel & $>50$ & Aquiclude \\
\hline
\end{tabular}




\subsection{Klima}

Syrien, mit seiner geographischen und klimatischen Lage, wird den Subtropen-Ländern zugeordnet. Im Allgemeinen sind die Sommer heiß und trocken und die Winter mild bis kühl und verhältnismäßig feucht. Sowohl der Sommer als auch der Winter dauert ungefähr vier Monate. Die Kernmonate des Sommers sind Juli und August. Die zweite Maiund die erste Oktoberhälfte hat in vielen Fällen noch sommerliche Witterung. Gegensätzlich sind Januar und Februar Kernmonate des Winters. Im Winter wird Syrien hauptsächlich von den Zyklonen beeinflusst, die über dem Mittelmeer gebildet werden oder vom atlantischen Ozean kommen. Diese Zyklonen sind mit zunehmender Bewölkung, absinkenden Temperaturen, Niederschlag, und kaltem nordwestlichem oder nordöstlichem Wind begleitet. Demzufolge wird sowohl die Ein- wie die Rückstrahlung vermindert. Außerdem ist das Klima von Syrien im Winter manchmal auch von einem Ausläufer der sibirischen Antizyklonen beeinflusst, der für das kalte wolkenlose Wetter verantwortlich ist. Demgegenüber liegt Syrien im Sommer im Bereich der subtropischen Trocken- und Passatzonen. Dies bedeutet, dass das trockene und warme Sommerwetter von großer Beständigkeit ist. Hierfür ist wolkenloses Wetter mit hohen Lufttemperaturen und der Herrschaft trockener südwestlicher Winde typisch für den Sommer. Die Bewölkung im Sommer ist im Gegensatz zum Winter sehr gering, welche für eine starke Sonneneinstrahlung tagsüber und eine kräftige nächtliche Abstrahlung verantwortlich ist und zu hohen Temperaturdifferenzen im Tagesgang führt. Die meisten Niederschläge fallen in den Kernmonaten des Winters. Die geographische Verteilung der Niederschläge wird durch die orographische Gestaltung stark beeinflusst. die Niederschläge nehmen von Westen nach Osten des Lands ab (Abb. 2-5). Im Gegensatz zum westlichen Landesteil (Küstengebirge) haben die Niederschläge im Osten episodischen Charakter. Die Luftfeuchtigkeit verringert sich unter den orographischen Einfluss von Westen nach Osten und die Temperatur fällt. Entsprechend ist die mittlere jährliche Verdunstung in den östlichen Bereichen des Landes viel höher als in westlichen Bereichen (insb. im Vergleich zu den Küstengebieten).

Die jährlichen Niederschläge im betrachteten Gebiet variieren zwischen ca. $150 \mathrm{~mm}$ nördlich der Palmyra-Ketten und ca. 600 mm nordwestlich von Aleppo (Abb. 2-6). Dieser Niederschlag beruht darauf, dass dieses Gebiet im Nordwesten und im Westen durch die ozeanischen Klimabedingungen beeinflusst wird, während in Richtung Südosten der Einfluss des kontinentalen Klimas allmählich zunimmt. 


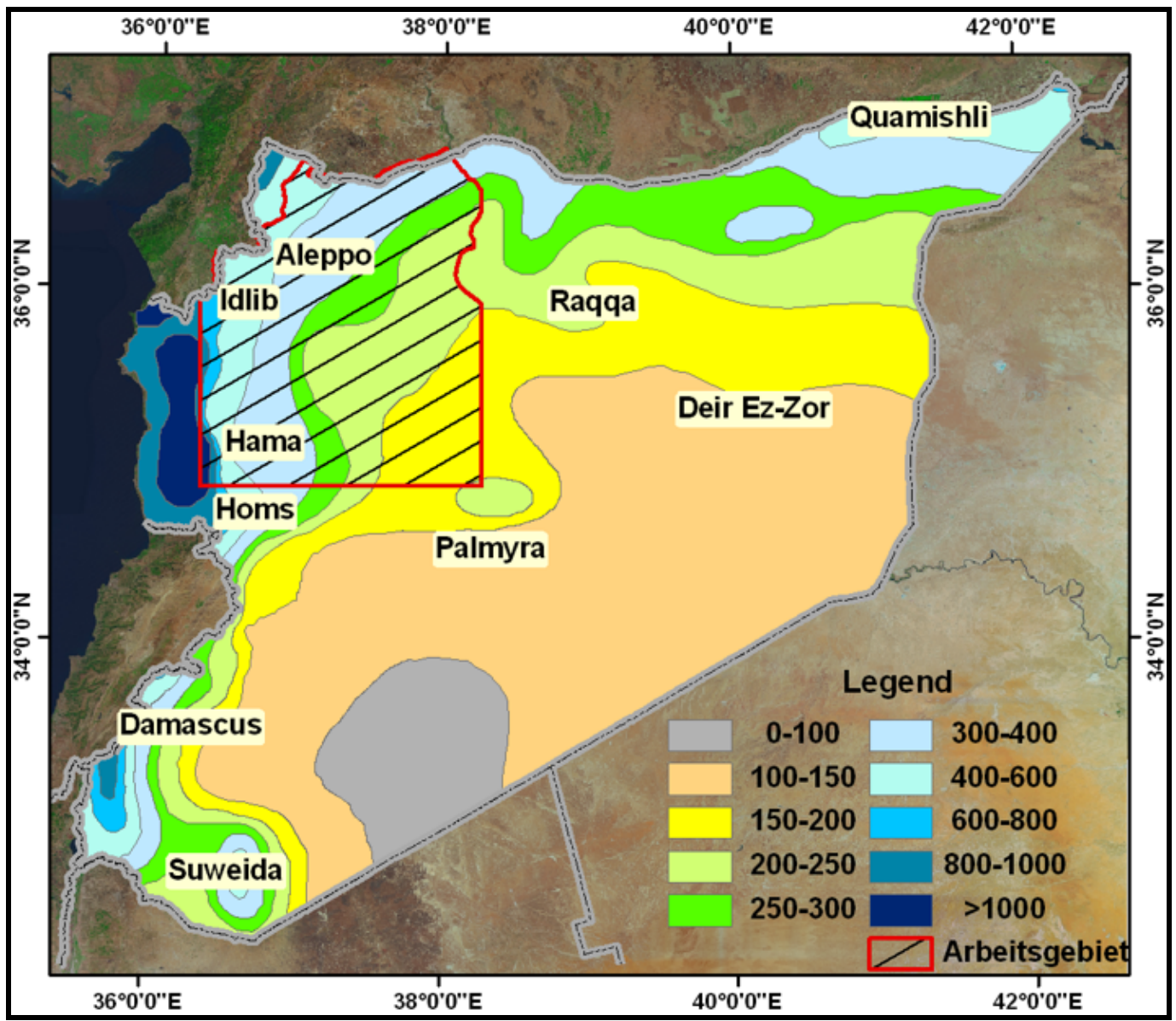

Abb. 2-5: Die jährlichen Niederschläge in mm (Meteorologie-Abteilung, Damaskus)

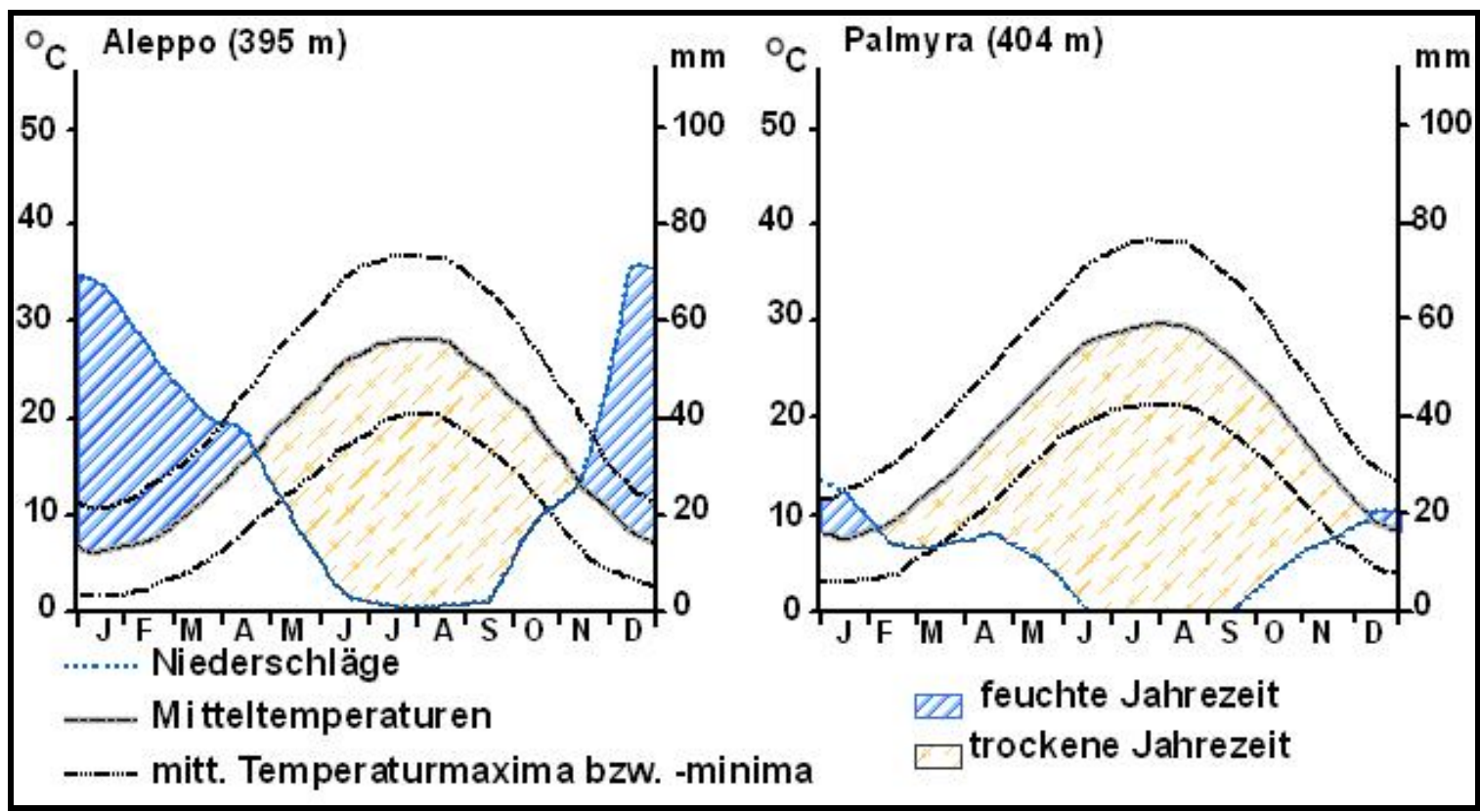

Abb. 2-6: Klimadiagramme von Aleppo \& Palmyra (geändert nach RöSNER, 1995) 


\subsection{Böden}

Überall in Syrien bzw. in dem betrachteten Gebiet werden die Böden vor allem durch vier Faktoren bestimmt: Ausgangsgestein, Klima, Bodengenese und menschliche Einwirkungen. Unter dem Einfluss des letzten Faktors entwickelten sich die westlichen Teile des Gebiets wegen der Degradierung oder Vernichtung der Waldbestände zu nackten Karstflächen. Diese Teile waren vermutlich bis zur Zeit der römisch-byzantinischen Antike von mächtigen Böden überdeckt (WIRTH, 1971). Östlich von dieser s. g. Einöde, in den Beckenlandschaften von Aleppo und in der Gegend von Homs bis Hama befinden sich rote bis dunkelrötliche kalkreiche tiefgründige Böden besonderer Fruchtbarkeit (Grumusole) in größeren zusammenhängenden Flächen. Diese Böden haben sich vor allem über den Mergeln des Senons und Alttertiärs während eines wohl feuchteren und wärmeren Klimas ausgebildet. Nach VAN LIERE (1960/61) sollen diese Böden während ihrer Bildung einen Entkalkungsprozess erfahren haben; der jetzige Kalkgehalt sei auf jüngere äolische Ablagerung von Kalkstaub zurückzuführen. VAN LIERE vermutete, dass es sich um Reliktböden bzw. umgelagerte fossile Böden handelt.

Der zweite Bodentyp wird durch vulkanische Böden des Jebel Hiss und Jebel Shbith sowie Basalt von Hama gebildet. Die Böden östlich und südöstlich des betrachteten Gebiets bis zum Euphrat im Osten sind über der miozänen Gips- und Anhydritformation gebildet. Die geomorphologische Lage dieser Böden in den Binnenbecken mit relativ hochstehendem und mit Salzen belastetem Grundwasser begünstigt die Gips- und Salzanreicherungen. Diese Böden sind teilweise mit Gipskrusten bedeckt. Auch bei reichlicher Bewässerung bieten diese Böden ungünstige Wuchsbedingungen und mindern damit den Ertrag.

Mit den oben genannten Bodentypen findet man vergesellschaftete Yellow Soils nämlich auf ebeneren Flächen und Plateaus, insbesondere in dem Bereich zwischen den Basaltplateaus bzw. den abflusslosen Becken und den Palmyra-Ketten im Süden. Während Yellow Soils aus Basalt kalkarm bis kalkfrei sind, ist diese Art von Böden wie Grumusole als kalkhaltig zu bezeichnen. Diese Böden erreichen eine mittlere Entwicklungstiefe und sind charakterisiert als braune bis gelbe schluffig-lehmige lockere Böden. 


\subsection{Vegetation und Landnutzung}

Die Vegetation in dem Gebiet ist wie auch in allen anderen Bereichen Syriens durch anthropogene Eingriffe verändert und drastisch degradiert worden. Hierzu hat auch die Klimaänderung beigetragen. Gebirgige Bereiche in dem betrachteten Gebiet waren in der Vergangenheit flächenhaft bewaldet. Doch sogar auf den topographischen Karten von 1940 (siehe Datengrundlage), die auf den von der französischen Mandatsregierung erstellten Karten basieren, sind Merkmale für Wald auf dem Plateau von Missyaf und der nördlichen Palmyra-Ketten zu finden. РАВОт (1956) berichtete von vereinzelten alten Gehölzformationen auf den Höhen des J. Bilass inmitten der syrischen Wüstensteppe (Abb. 2-7).

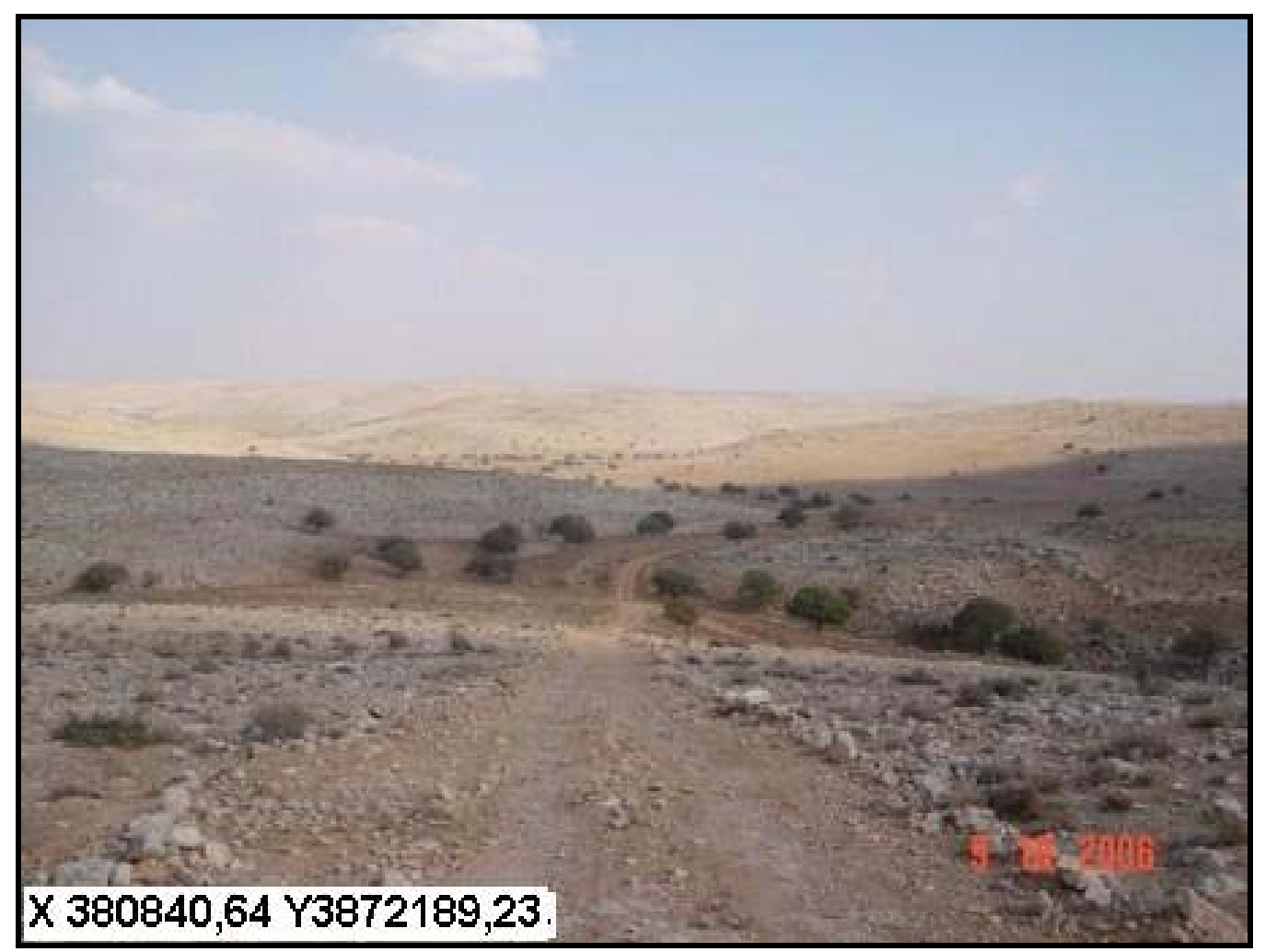

Abb. 2-7: Vereinzelte Bäume von Pistascia atlantica in den Wadis innerhalb J. Bilass

Die pflanzengeographische Gliederung von Syrien lässt das Gebiet in vier Zonen umfassen (Abb. 2-8). Bezüglich des Ackerbaus liegt ein großer Bereich des Untersuchungsgebiets im Südosten aufgrund des klimatisch bedingten Bewuchses in einem typischen Nomadengebiet. Nach Westen und Nordwesten, in Richtung des kultivierten Lands Syriens, dehnt sich der Ackerbau aus. Bewässerungsfelder und Regenfelder sind überall vorhanden. Historisch waren Regenfelder weit verbreitet (WIRTH, 1971). Demgegenüber hat sich bewässerte Landwirtschaft erheblich seit der Erfindung der Dieselpumpe ver- 
breitet. Während im Südosten und im Zentrum des betrachteten Gebiets der Regenfeldbau und der Bewässerungsanbau in Wadi-Gärten vorherrschen, dominieren im Westen und Nordwesten die bewässerten Felder neben Pistazien, Ölbaum- und Weinkulturen.

In den Wadi-Gärten wird überwiegend Gemüse (Zwiebeln, Tomaten, Gurken, u. a.) angebaut. Die lokale landwirtschaftliche Produktion hier reicht jedoch meistens nur für den Eigenbedarf der Bauernfamilien aus. Nach Westen ist eine Intensivierung der Landwirtschaft zu beobachten. Als Wintergetreide ist hier der Anbau von Weizen und Gerste verbreitet. Sommerkulturen sind vor allem Baumwolle und Melonen bzw. Wassermelonen, gefolgt von Tomaten, Paprika und Gurken etc. Diese können als Bewässerungs- oder Regenfluren eintreten. Auch der Kartoffelanbau ist überall weit verbreitet. Im Zentrum des Gebiets ist die Intensivierung der Landwirtschaft aufgrund der zunehmenden Degradation im Bereich der Vegetation und der Böden sehr bedenklich. Infolge der unsicheren Klimaverhältnisse führt dies zu unabwägbaren Risiken, da eine künstliche Bewässerung wegen der hydrologischen Situation nicht sinnvoll ist.

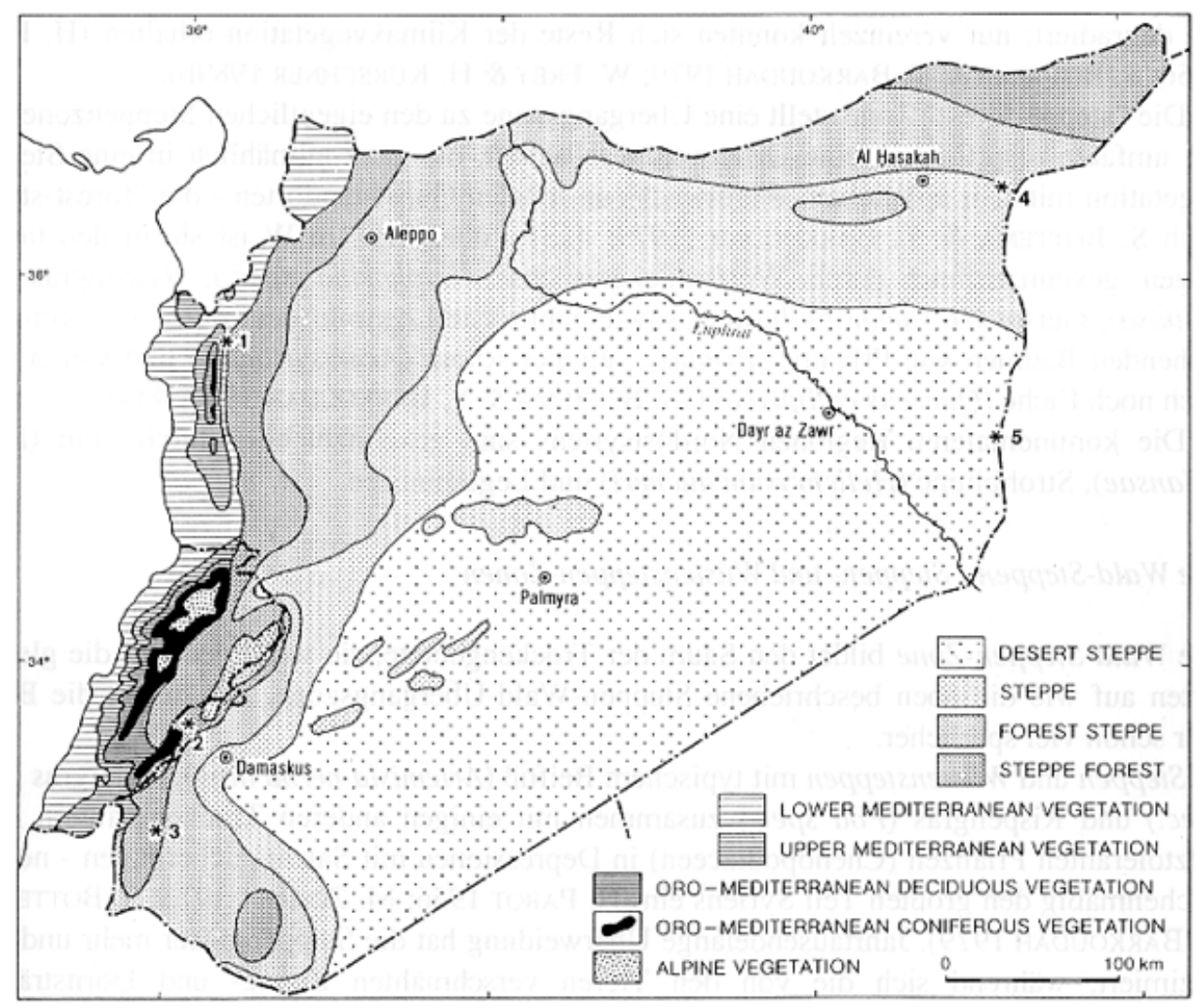

Abb. 2-8: Pflanzengeographische Gliederung Syriens (ВоттEMA, 1979) 

Ka p it e 13

Fernerkundungs-Missionen 


\section{Fernerkundungs-Missionen}

Fernerkundung basiert auf der Technologie der Photogeologie, die seit 1930 etabliert ist. Satellitenmissionen, Neu- und Weiterentwicklung der analogen und digitalen Aufnahmesysteme sowie die Fortschritte in der elektronischen Datenverarbeitung ermöglichen heute eine Erderforschung ausgehend von Höhe der Flugzeugflüge bis zum Orbit der geostationären Satelliten mit verschiedenen Auflösungen. Die Satellitensysteme können aus einer größeren Entfernung berührungsfrei die Reflexionen elektromagnetischer Wellen der Erdoberfläche und auf ihr befindlicher Objekte messen. Aus diesem Datenmaterial können dann je nach Fragestellung und diesbezüglich eingesetzter Messtechnik Informationen abgeleitet werden. Die Wellenbereiche des elektromagnetischen Spektrums gehen von dem für den Menschen visuell sichtbaren Bereich (VIS) über das nahe Infrarot (NIR) und das thermale Infrarot (TIR) bis hin zu Radarsystemen. Die optischen Systeme, die das sichtbare Licht bis zum Infrarot erfassen, brauchen freie Sicht, sie können Wolken nicht durchdringen (Abb. 3-1). Diese stören dagegen nicht die abbildenden Radarsysteme, die allerdings den Nachteil nicht so detaillierter Abbildungen haben. Im Allgemein werden die Sensoren so gebaut, dass sie für einen bestimmten Wellenlängenbereich innerhalb eines atmosphärischen Fensters sensibilisiert sind.

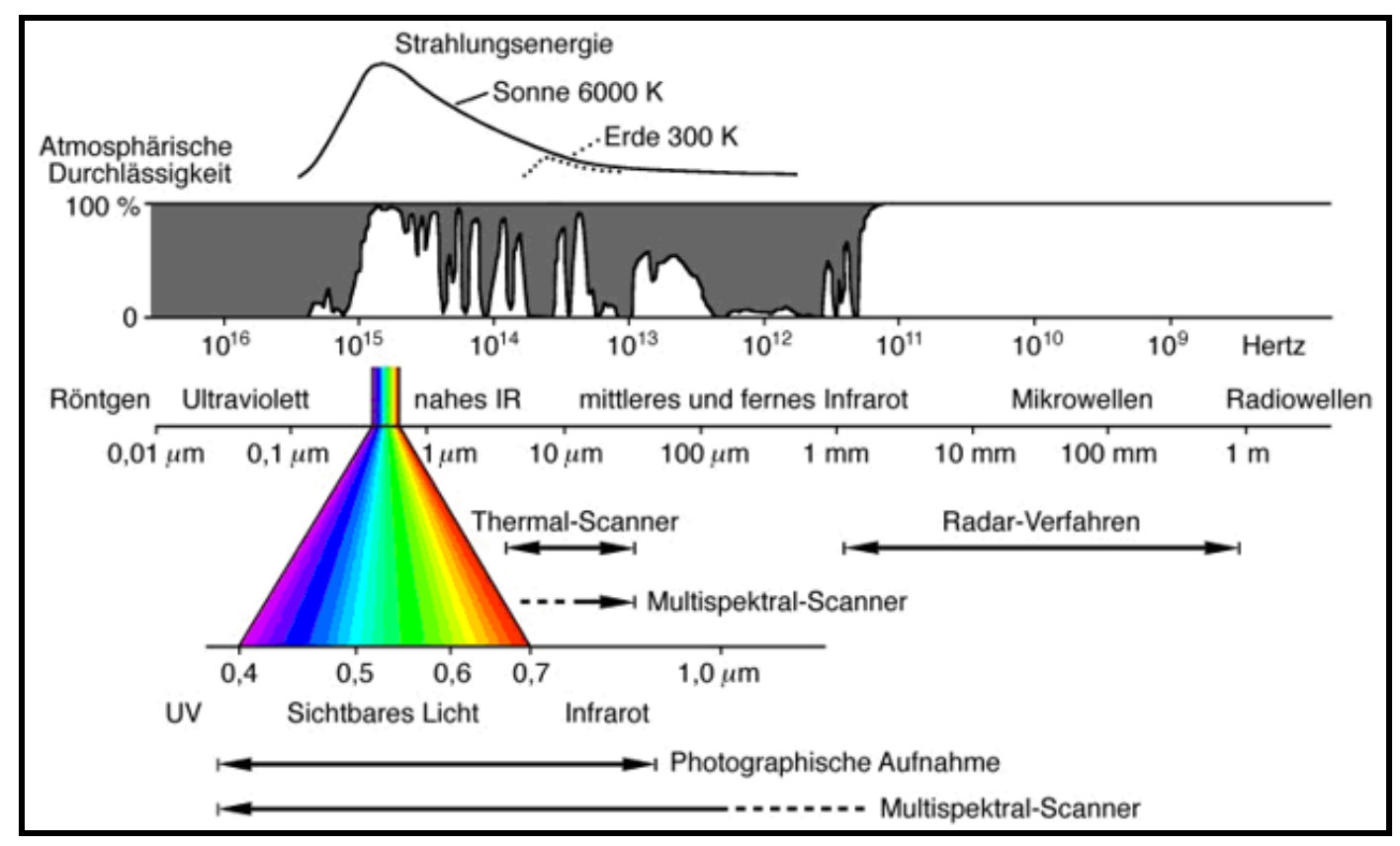

Abb. 3-1: Das elektromagnetische Spektrum (ALBERTZ, 2001) 


\subsection{Landsat-Mission}

Seit 1960 werden Wettersatellitensysteme für die Überwachung der Erdoberfläche benutzt. Allerdings sind diese nicht für das ausführliche Kartieren der Landoberfläche optimiert. Der erste Satellit, der spezifisch dafür gedacht war, wurde von der USAWeltraumbehörde NASA 1972 in Form der Landsat-Mission (Landsat-1) betrieben. Seitdem stehen den Geowissenschaftlern digitale Satellitenbilddaten über nahezu sämtliche Regionen der Erde zur Verfügung. Die Sensoren der Landsat-Mission wurden dadurch entwickelt, dass sie den visuellen Bereich, den Spektrumsbereich des thermalen Infrarots, sowie auch den Mikrowellenbereich abdecken und zur Erdbeobachtung eingesetzt werden.

Bis Ende 1983 waren die Landsat-1 bis Landsat-3 im Einsatz, welche mit dem optomechanischen MSS (der multispektrale Scanner) ausgerüstet sind. Der MSS nimmt Strahlung mit 4 Kanälen im sichtbaren Bereich (VIS) und im Bereich des Nahen Infrarots (NIR) auf. Ab Juli 1982 wurde die zweite Generation mit Landsat-4 und -5 betrieben. Neben dem MSS hat diese Landsat-Generation einen neuen Sensor an Bord, der in seiner spektralen und räumlichen Auflösung verbessert wurde. Dabei handelt es sich um den TM (Thematic Mapper), ein ebenfalls optomechanisches System (Tab. 3-1).

Die jüngste Generation der Landsat-Serie ist der Landsat-7 Satellit, der mit dem Enhanced Thematic Mapper (ETM+) ausgestattet ist. Dieser Sensor besitzt eine räumlich höhere Auflösung im thermalen Infrarot (TIR) und einen zusätzlichen panchromatischen Kanal höherer Auflösung im sichtbaren Spektralbereich (VIS).

Die multispektralen Eigenschaften der Landsatdaten ermöglichen den Einsatz von Landsatbilder in mehreren Bereichen. In der Tabelle 3-2 sind die Hauptnutzungsbereiche von Aufnahmen der einzelnen Spektralkanäle des ETM+ aufgelistet.

Eine detaillierte Darstellung der Aufnahmeprinzipien der Landsat-Mission ist ausführlich z.B. in Kronberg, 1985, SABins (1997) und Richards \& JiA (1999) beschrieben. Ausführungen zu den strahlungstheoretischen Grundlagen in der Fernerkundung finden sich z.B. bei KRAUS (1990). 


\subsection{ASTER - Mission}

ASTER (Advanced Spaceborne Thermal Emission and Reflection Radiometer) ist eines der fünf wissenschaftlichen Instrumente der Terra Plattform. Dieser Satellit wurde am 18. Dezember 1999 von der NASA gestartet. Das in Japan gebaute Instrument zeichnet seit dem Februar 2000 Daten auf. Es umrundet in einem nahpolaren Orbit die Erde in einer Höhe von 705 km (Tab. 3-3). Das Sensorsystem besteht aus drei Sensoren (Abb. 3-2). Der VNIR Sensor (Visible and Near Infrared Radiometer) besitzt drei Kanäle mit einer Auflösung von 15m. Der SWIR Sensor (Short Wave Infrared Radiometer) hat eine geometrische Auflösung von 30m in sechs Spektralbereichen. Der dritte Sensor ist der TIR (Thermal Infrared Radiometer), der aus fünf Kanälen mit einer geometrischen Auflösung von $90 \mathrm{~m}$ besteht. Eine ASTER-Szene deckt eine Fläche von 60 x 60 Kilometer ab.

Zwecks Ermittlung der Karten, der Temperaturverteilung, der Emissivität, der Reflektion und der Höhenverteilung sammeln die 14 Bänder Daten. Sie ermöglichen dadurch ein gutes Verständnis für die Wechselwirkung zwischen der Biosphäre, der Hydrosphäre, der Lithosphäre und der Atmosphäre.

Der Nahinfrarot-Kanal 3 ist doppelt vorhanden. Der erste Kanal 3N (Nadir) bildet wie die übrigen auch die Erdoberfläche senkrecht unter der Plattform ab. Der zweite Kanal 3B nimmt dieselbe Fläche etwas später um $27,6^{\circ}$ rückwärts gedreht auf. Auf diese Weise entsteht aus den beiden im Band 3 aufgenommenen Levels 1A Daten ein Stereobild, das durch Nutzung geeigneter Computerprogramme zur Berechnung eines Digitalen Höhenmodells (DHM) herangezogen werden kann.

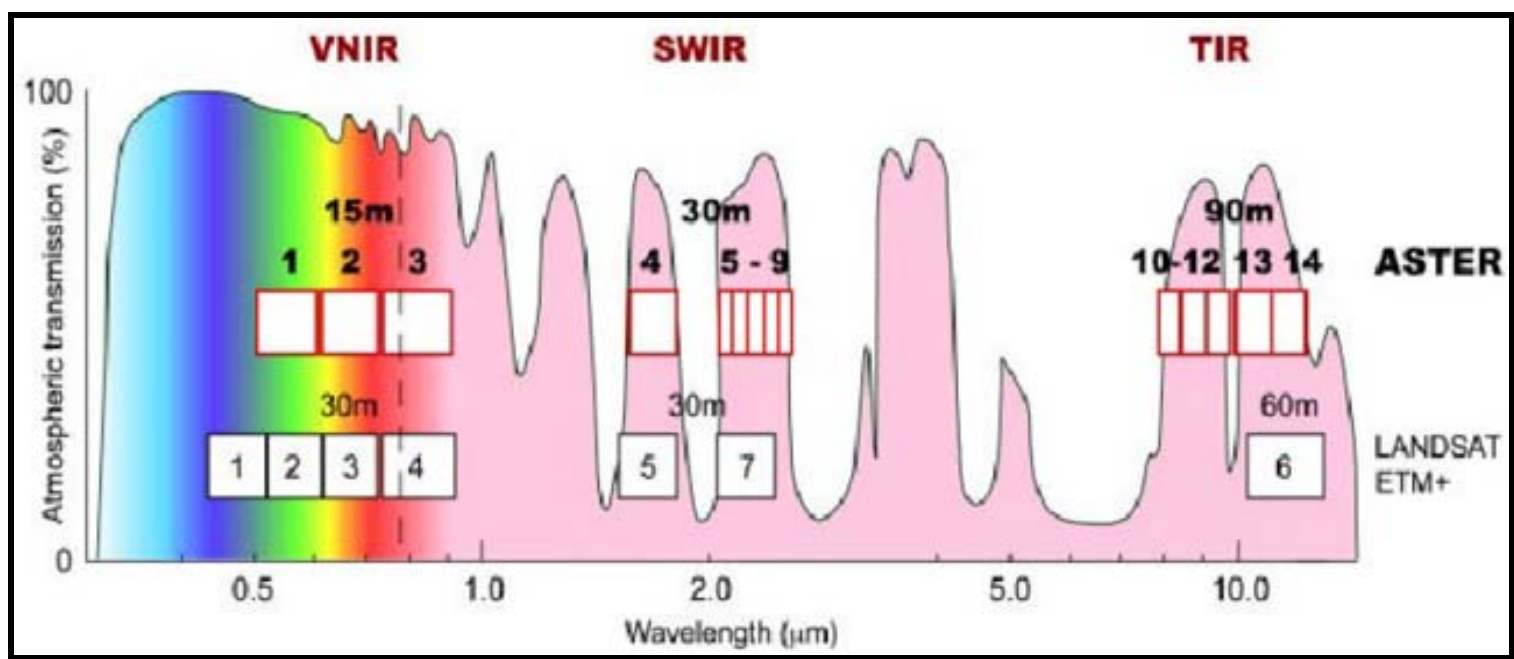

Abb. 3-2: Spektralbereichen der ASTER und ETM+ Daten (PCIGEOMATICS, 16.11.2007) 
Tab. 3-3: Die Eigenschaften der ASTER Bänder

\begin{tabular}{|c|c|c|c|}
\hline Charakteristikum & VNIR & SWIR & TIR \\
\hline \multirow{5}{*}{ Spektralraum } & Band 1 & Band 4 & Band 10 \\
& $0.52-0.60 \mu \mathrm{m}$ & $1.600-1.700 \mu \mathrm{m}$ & $8.125-8.475 \mu \mathrm{m}$ \\
\cline { 2 - 4 } & Band 2 & Band 5 & Band 11 \\
& $0.63-0.69 \mu \mathrm{m}$ & $2.145-2.185 \mu \mathrm{m}$ & $8.475-8.825 \mu \mathrm{m}$ \\
\cline { 2 - 4 } & Band 3N & Band 6 & Band 12 \\
\cline { 2 - 4 } & $0.76-0.86 \mu \mathrm{m}$ & $2.185-2.225 \mu \mathrm{m}$ & $8.925-9.275 \mu \mathrm{m}$ \\
\cline { 2 - 4 } & Band 3B & Band 7 & Band 13 \\
& $0.76-0.86 \mu \mathrm{m}$ & $2.235-2.285 \mu \mathrm{m}$ & $10.25-10.95 \mu \mathrm{m}$ \\
\cline { 2 - 4 } & & Band 8 & Band 14 \\
& & $2.295-2.365 \mu \mathrm{m}$ & $10.95-11.65 \mu \mathrm{m}$ \\
\cline { 2 - 4 } & & Band 9 & \\
\hline \multirow{4}{*}{ Auflösung } & $15 \mathrm{~m}$ & $360-2.430 \mu \mathrm{m}$ & $90 \mathrm{~m}$ \\
\hline
\end{tabular}

\subsection{SRTM - Mission}

Die Shuttle Radar Topography Mission (SRTM) startete im Herbst 1999. Dabei handelte es sich um ein Gemeinschaftsprojekt zwischen USA (NASA-JPL, NIMA), Deutschland (DLR) und Italien (ASI). Das Ziel des Projekts war, die Topographie der gesamten Erdoberfläche dreidimensional mit Hilfe von Radarsensoren zu kartieren.

Das Radarsystem der SRTM-Mission besteht aus zwei Einheiten X-SAR und SIR-C. Die Erste ist das in deutsch-italienischer Kooperation entwickelte X-SAR-Radar, welches im s. g. X-Band bei einer Wellenlänge von 3,1 cm bzw. einer Frequenz von 9,6 Gigahertz arbeitet. Die Zweite ist das amerikanische SIR-C-Radar. Dieses arbeitet im C-Band bei einer Wellenlänge von $6 \mathrm{~cm}$ bzw. einer Frequenz von 5,3 Gigahertz. Die SRTM umrundet auf einer Höhe von $233 \mathrm{~km}$ unseren Planeten. Die Bahnneigung der Umlaufbahn gegenüber dem Äquator beträgt 57 Grad. Mit dieser Bahnneigung können Gebiete zwischen 60 Grad Nord und 56 Grad Süd erreicht werden. Während das Shuttle in seiner Umlaufbahn kreist, dreht sich die Erde weiter und so werden nach und nach alle Kontinente überflogen. Das amerikanische C-Band-Radar tastet den Erdboden in $225 \mathrm{~km}$ breiten Streifen ab und erfasst somit die gesamte überflogene Landfläche. Das deutsch-italienische X-SARSystem nimmt $50 \mathrm{~km}$ breite Streifen der Erdoberfläche auf und kartiert damit 40 Prozent der überflogenen Landfläche, aber dafür mit einer deutlich besseren Höhen-Auflösung. 
Die SRTM-Mission beruht auf der Interferometrietechnik. Diese Technik beschäftigt sich mit der Phaseninformation der Rückstreuung. Die Phase ist sehr sensitiv gegenüber Entfernungsänderungen zwischen dem Objekt und der Aufnahmeplattform. Für eine interferometrische Verarbeitung müssen mindestens zwei Datensätze eines Zielgebietes vorliegen. In den zwei Aufnahmen wird dasselbe Bodensegment aus unterschiedlichen Perspektiven aufgezeichnet. Aus diesen Aufnahmen können interferometrische Phasenunterschiede errechnet werden, die unter anderen zur Generierung von Höhenmodellen verwendbar sind. Zur detaillierten Beschreibung des Prinzips der SAR-Interferometrie sei z.B. auf BAMLER \& HARTL (1998) verwiesen.

Entsprechend wird zwischen einer Repeat- und Single-Pass-Interferometerietechnik unterschieden. Bei dem Repeat-Single-Pass handelt sich um die SAR-Interferometrie, die eine relativ junge Disziplin zur Erfassung der Erdoberfläche darstellt. Zahlreiche interferometrisch gewonnene Höhenmodelle basieren auf SAR-Daten der Satelliten ERS-1 und -2. Die Umlaufbahnen beider Satelliten wurden als Tandemmission konzipiert, so dass im Abstand von wenigen Tagen ein Gebiet zweimalig mit unterschiedlicher Geometrie erfasst wird (dual- oder repeat-pass interferometry). Höhenmodelle aus ERS-1/-2-Daten unterliegen Ungenauigkeiten, da die Radarrückstreuung vor allem durch unterschiedliche atmosphärische Bedingungen während der beiden Aufnahmen beeinträchtigt wird.

Das Problem einer zeitlichen Dekorrelation der Daten entfällt hingegen bei SRTMMission. Hier war der erste Einsatz einer weltraumgestützten EinpassRadarinterferometrie (Single-Pass-Interferometer), womit die Erde erstmals gleichzeitig von zwei Antennen aufgenommen wurde. Um zwei Radarbilder von zwei leicht verschiedenen Positionen zu erhalten, ist eine Antenne in der Ladebucht untergebracht und die zweite ist am Ende eines $60 \mathrm{~m}$ langen Masts angebracht. Die Unterschiede in den aufgenommenen beiden Bildern erlauben es, die Höhenkurven des abgebildeten Gebietes zu berechnen (Abb. 3-3). Gegenüber optischen Stereobildern und anderen früheren Verfahren hat die SRTM einen entscheidenden Vorteil, dass sie unabhängig von Bewölkung und Tageslicht arbeitet, womit die Erzeugung digitaler Höhenmodelle der gesamten Erde in einem einheitlichen Maßstab und in sich homogen ermöglicht wurde. Beispielsweise in dem Fall der Repeat-Pass-Interferometerietechnik wurde die Rückstrahlung wegen der Änderungen an Vegetationslage, Bodenfeuchte und Luftmassen beeinflusst, welche bei einer so kurzen SRTM-Mission beseitigt sind. 
Die bereitgestellten SRTM-Daten werden in zwei Versionen angeboten. Eine ist SRTM-1 mit einer Auflösung von einer Bogensekunde (30m) in Länge und Breite bei 3601 und 3601 Pixeln. Die Zweite SRTM-3 (C-Band) wurde aus den SRTM-1 erzeugt mit einer Auflösung von 3 Bogensekunden (90m) bei 1201 x 1201 Pixel. Die Höhendarstellung erfolgt in Metern bis auf 6 Meter Genauigkeit. Die SRTM-3 steht durch eine Internetseite von USGS für die ganze Welt georeferenziert und kostenlos zur Verfügung. Das Referenzellipsoid ist WGS84. Die Daten liegen als Kacheln vor und werden als 16-bit signed integer Datensatz (Zahlbereich von -32768 bis 32767) in einem binären Datenformat .HGT (heightfield) abgelegt. Jede Kachel des DHMs hat nach der Geokodierung eine Größe von 1 Grad sowohl in Längen- als auch in Breitengradrichtung. Die Dateinamen beziehen sich auf die Länge und Breite der unteren linken Ecke des Datenfeldes, bei (N35E037) sind die Koordinaten des Pixels $35^{\circ}$ geographische Breite und $037^{\circ}$ geographische Länge.

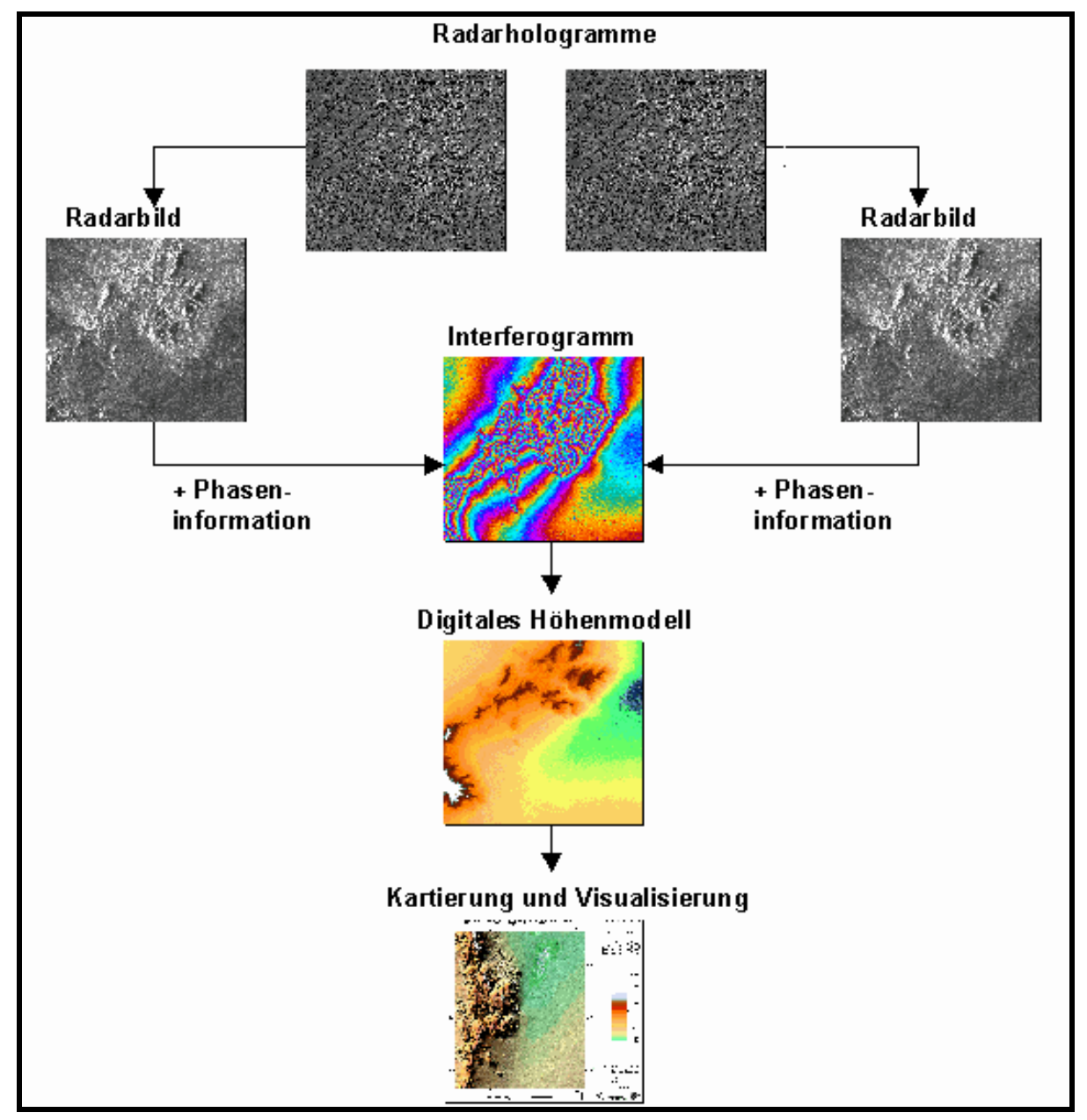

Abb. 3-3: Schematischer Aufbau eines Interferometrie-Prozessors (DLR, 02.12.2007) 
Es gibt einige wenige Problemzonen mit größeren Ungenauigkeiten der SRTM-Daten. Diese sind zum einen Wasserflächen, die wegen ihrer glatten Oberfläche sehr geringe Intensitäts- und Kohärenzwerte aufweisen, was $\mathrm{zu}$ einem Versagen der SARInterferometrie führt. Fehlpixel bedingt durch Wasser oder Eis haben den Höhenwert 32768. Deshalb sind Küstenlinien in SRTM-Daten unscharf dargestellt und größere Wasserflächen erscheinen uneben. Problemzonen können auch in der Nähe von sehr hellen Radarzielen entstehen, da dann bei der SAR Prozessierung eine Überstrahlung in benachbarte Pixel in Azimutrichtung stattfindet. Dies führt in den betroffenen Bereichen zu Artefakten, Dekorrelation und Versagen der SAR-Interferometrie. Wald- und Gebäudehöhen (Vegetationsdecke, Bebauung) sind mit den SRTM-Daten erfasst. Infolgedessen sind die aus den SRTM-Messungen abgeleiteten Produkte in der Regel Digitale Oberflächenmodelle (DOM) zu bezeichnen. Diesem liegt zugrunde, dass das Radar-Signal nur wenige Meter in die Vegetationsdecke eindringt (Abb. 3-4).

Durch Vergleich der Genauigkeit der SRTM Daten mit anderen Daten konnte nachgewiesen werden, dass die SRTM-Höhendaten im offenen freien Gelände über gute Genauigkeit verfügt. Sie können deshalb die Hauptaufgabe der Höhenmodelle in vollem Umfang erfüllen. Im Untersuchungsgebiet sind die anthropogenen Einflüsse auf das Relief und die Vegetationsbedeckung aufgrund ihrer Geringmächtigkeit in Bezug auf den Untersuchungsmaßstab vernachlässigbar. Dementsprechend kann das von SRTM für das Gebiet erzeugte Modell als DHM bezeichnet werden.

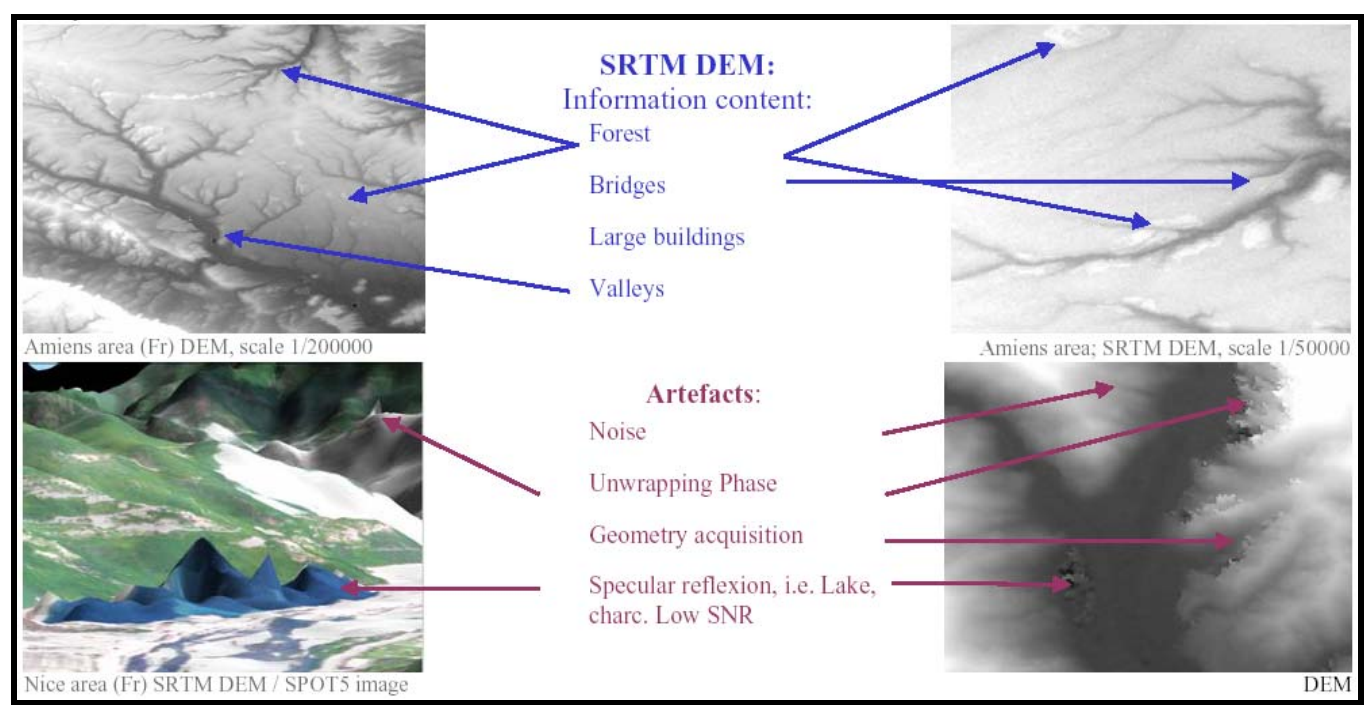

Abb. 3-4: Inhalt der SRTM digitalen Höhendaten (MAIRE \& DATCU, 2004) 


\subsection{Einsatz von Fernerkundungsdaten in den Geowissen- schaften}

Die Fernerkundungsdaten finden seit mehreren Jahren eine verstärkte Anwendung in verschiedenen geowissenschaftlichen Teilgebieten. Mit der Entwicklung von neuen Sensoren standen unterschiedliche Möglichkeiten der Erdbeobachtung und Analyse für Disziplinen wie Geographie und Geologie bzw. Geomorphologie zur Verfügung. Mit ihrer Fähigkeit, ausgedehnte Gebiete der Erdoberfläche synchron zu erfassen, bieten die Fernerkundungsdaten ein wertvolles Instrument beim Studium geomorphologischer/geologischer Züge der Erdoberfläche. Im Gegenteil zu den Luftbildern, die nur kleinere Gebiete erfassen können, ermöglichen die Fernerkundungsdaten die Erkundung der großen geomorphologischer/geologischen Strukturen durch die Kombination verschiedener geologischer Relief-, Vegetations-, Mineralisations- und weiterer Merkmale (Photogeologische Kriterien) (Abb. 3-5). Sie sind damit dafür geeignet, große geomorphologische/geologische Einheiten zu erfassen; Gesteine zu klassifizieren; vulkanische Gegenden zu kartieren; Mineral, Erdöl und Quellen zu erkundigen; Geomorphologische Karten zu erstellen und regionalgeologische Strukturen zu erkennen.

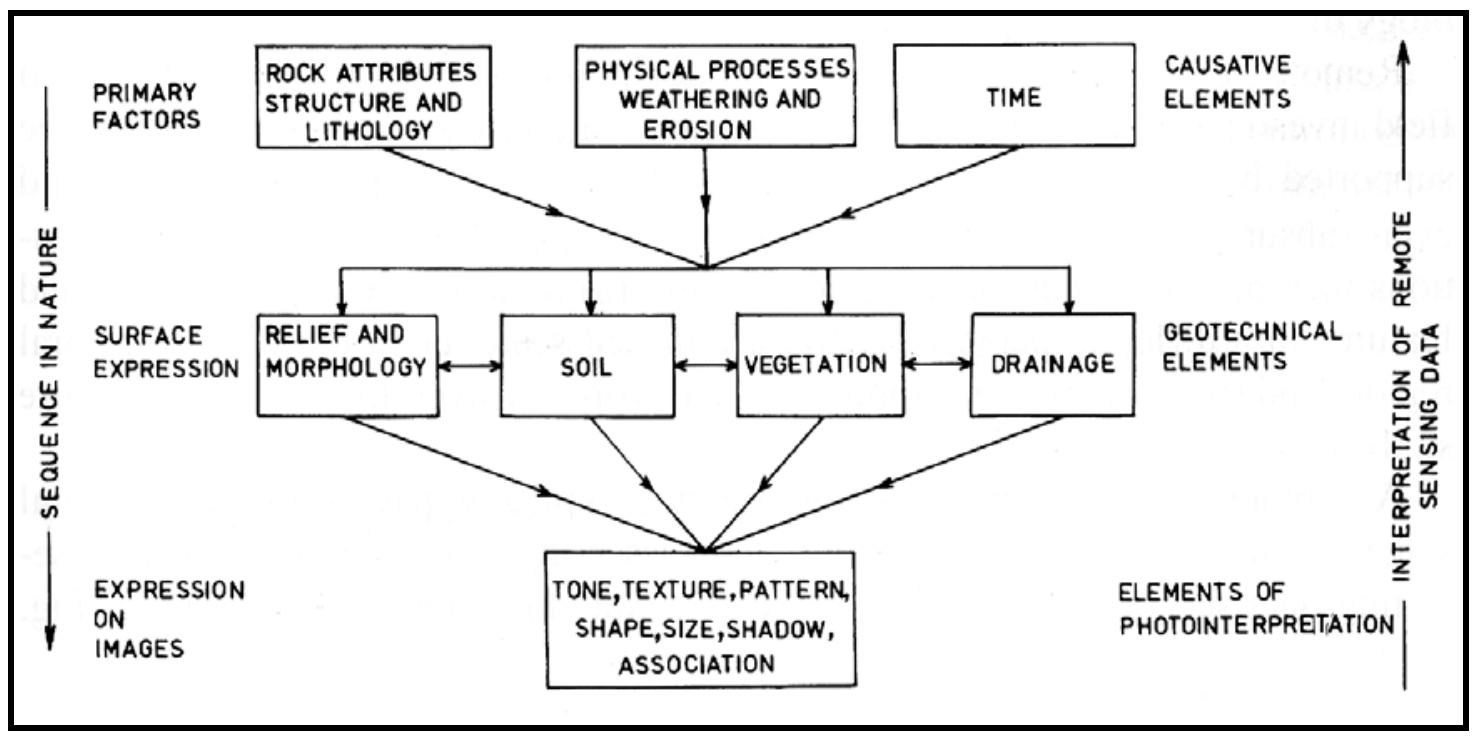

Abb. 3-5: Verhältnis zwischen den geoökologischen Elementen und den Elementen der Photointerpretation (GUPTA, 2003) 
Die erste große Entwicklung zur Anwendung von Fernerkundung in der Geomorphologie war mit dem Einsatz multispektraler Scanner (passive Systeme) verbunden. Sowohl die einzelnen Bänder als auch die Kombination von mehreren Bändern enthalten viele Informationen über die Geomorphologie. Hierbei können mittels der Fernerkundungsdaten die großräumigen Bauelemente der Erdoberfläche bis zu solchen globalen Dimensionen identifiziert werden, welche mittels anderer Methoden (Feldforschung oder Luftbilder) nur schwierig in ihrer Gesamtausdehnung beurteilt werden können.

Aktive Aufnahmesysteme sind ebenfalls für geomorphologische Fragestellungen sehr dienlich. Diese Systeme sind in mehreren Situationen von Vorteil, z. B. in Gebieten, wo Wolkenbedeckung und Aerosole oder Vegetation die Oberfläche verdecken. Die höhere Eindringtiefe der Mikrowellenstrahlung in das zu untersuchende Gestein bei aktiven Aufnahmesystemen kann hier auch von Vorteil sein.

$\mathrm{Zu}$ den geomorphologischen/geologischen Anwendungen zählt die geomorphologische Darstellung bzw. Kartierung aus Fernerkundungsdaten. Dafür hat sich die ursprünglich für den Zweck der Landnutzungsplanung entwickelte Landsat-Mission als äußerst nützlich erwiesen (DRURY, 1998). Die multispektrale Aufnahmeweise der Landsat-Sensoren gestattet es, in vegetationsarmen, semiariden oder ariden Gebieten eine Differenzierung von direkt zu Tage tretenden Gesteinseinheiten anhand ihrer charakteristischen spektralen Signaturen vorzunehmen (LIST, 1969). Zu dieser Anwendung gehört auch die Analyse von Flussnetzsystemen, die in kleineren Maßstäben regional mit multispektralen Daten wie Landsatdaten durchgeführt werden können.

Die Anwendung dieser Daten für die geomorphologischen/geologische Auswertung war erfolgreich, obwohl die Multispektralsensoren der Landsat-Serie aufgrund der Position im elektromagnetischen Spektrum und der spektralen Bandbreite ihrer Aufnahmeempfindlichkeit nur Identifizierungen einzelner Mineralgruppen, wie Hydroxide, FeVerbindungen und Karbonate zulassen. In den Fällen, in denen die spektralen Eigenschaften von Gesteinseinheiten durch die Bildung von gering mächtigen Böden bzw. Regolith oder durch oberflächliche Hartkrustenbildungen maskiert sind, werden aufgrund der Beziehung zwischen oberflächlichen Verwitterungsbildungen und unterlagernden Lithologien Rückschlüsse auf die Verbreitung und Lagerung von Gesteinseinheiten gezogen. Auch indirekte naturräumliche Faktoren wie die Verbreitung unterschiedlicher Vegetationsarten werden als Hinweise auf die Geologie eines Untersuchungsgebietes 
genutzt. Neben den Kriterien, die bei der geomorphologischen/geologischen Auswertung von Luftbildern zur Interpretation von Objekten herangezogen werden - wie z.B. dem Grauton, der Textur und dem Homogenitätsgrad einer Oberfläche oder dem Erkennen von linearen Merkmale, enthalten die Fernerkundungsdaten wie Landsat und ASTER mit ihrer spektralen Information weitere wichtige Interpretationsfaktoren, z. B. durch gezielte Bildverarbeitungsprozesse können Variationen im Reflexionsverhalten oberflächennaher Objekte hervorgehoben werden.

Höhendaten zur Erstellung eines digitalen Höhenmodells (DHM) werden aus RadarInterferometrie und aus direkten Höhenmessungen mit LIDAR und RADAR gewonnen. Die Verwendung von digitalen Höhenmodellen (DHMs) eröffnet im Hinblick auf die geologische/geomorphologische Darstellung bzw. Kartierung zahlreiche Anwendungsmöglichkeiten. Insbesondere in Gebieten, deren Morphologie durch geologische Faktoren wie der unterschiedlichen Verwitterungsbeständigkeit von Gesteinseinheiten sowie durch strukturgeologische Elemente bestimmt wird, stellt die genaue digitale Wiedergabe der Topographie in einem DHM die Grundlage für eine morphotektonische Analyse dar.

Eine weitere Möglichkeit besteht in der Kombination von digitalen Höhenmodellen mit Fernerkundungsdaten. Durch die Integration der digitalen Höhendaten in einem Geographischen Informationssystem (GIS) ist es möglich geworden, geländeabhängige Prozesse zu modellieren, zu analysieren und zu visualisieren. Dabei können z. B. wichtige geomorphometrische Parameter (Wölbung, Hangneigung, Exposition) berechnet werden. Die Bedeutung dieser geomorphometrischen Parameter liegt darin, dass sie in Wechselwirkung mit den geomorphologischen Prozessen stehen. Die Prozesse verändern die geomorphologischen Merkmale und diese wiederum regeln die Prozesse, z. B.: Je steiler ein Hangabschnitt ist, umso intensiver ist die Abtragungswirkung des rinnenden Wassers. Andererseits differenzieren die Hangneigung und die Exposition das Strahlungsklima und den Bodenwasserhaushalt, die wiederum andere Prozesse im Landschaftshaushalt regeln.

Zur Interpretation von Luftbildern und digitalen Bildverarbeitung von Satellitenbilddaten für geologische Fragestellungen sei auf KRONBERG (1985), DRURY (2001) und BERGER (1994) verwiesen. 
- 36 - 
Ka p i t e 14

\section{Datengrundlage und die digitale A u f b e r e it ung}




\section{Datengrundlage und die digitale Aufbereitung}

Das Untersuchungsgebiet umfasst die ganze Provinz von Aleppo und große Teile vier anderer Hauptprovinzen Syriens (Idlib, Hama, Homs und Raqqa). Als Datengrundlagen stehen verschiedene Karten und Landsatszenen zur Verfügung, darüber hinaus SRTM Daten mit einer Auflösung von 90 m für das gesamte Gebiet. Diese Daten werden in den Tabellen 4-1, 4-2 und 4-3 dargestellt. Für die Durchführung dieser Arbeit sowohl für die Auswertungen und Interpretationen als auch für die Geländeuntersuchungen ist die Bereitstellung dieser Daten eine grundlegende Voraussetzung. Dies gilt uneingeschränkt ebenso für die Realisierung aller anderen geländebezogenen Tätigkeiten dieser Arbeit, wie die Probenahme.

\subsection{Die topographischen und geologischen Karten}

Die Ausdehnung des Untersuchungsgebiets ist hauptsächlich von drei topographischen Karten im Maßstab 1:200.000 Aleppo II, Hama IX und Salamiyeh XX abgedeckt. Das Schichtlinien-Intervall der Karten beträgt 20m. Kleinere Bereiche befinden sich auf den Karten III, XXI, XIII, XIV und XV (Abb. 2-1). Diese dargestellte Aufteilung ist mit den geologischen Karten im Maßstab 1:200.000 identisch (Tab. 4-1).

Tab. 4-1: Grundlagekarten für das Untersuchungsgebiet

\begin{tabular}{|c|c|c|c|}
\hline Karten & Maßstab & $\begin{array}{c}\text { Herst.- } \\
\text { Datum }\end{array}$ & Hersteller \\
\hline Topographische & $1: 200.000$ & $1938-1940$ & Deutschland \\
\hline Topographische & $1: 200.000$ & $1961-1984$ & Sowjetunion \\
\hline Geologische & $1: 200.000$ & 1964 & Sowjetunion \\
\hline Geomorphologische & $1: 1.000 .000$ & 1963 & Sowjetunion \\
\hline Bodenkarte & $1: 1.000 .000$ & 1980 & ACSAD \\
\hline $\begin{array}{c}\text { Karte der lithologi- } \\
\text { schen Einheiten }\end{array}$ & $1: 500.000$ & 1982 & Sowjetunion \\
\hline $\begin{array}{c}\text { Karte der Hydrogeo- } \\
\text { logie und Zonensa- } \\
\text { nierung }\end{array}$ & $1: 500.000$ & 1982 & Sowjetunion \\
\hline $\begin{array}{c}\text { Karte des oberen } \\
\text { Grundwasserleiters }\end{array}$ & $1: 1.000 .000$ & 1964 & Sowjetunion \\
\hline $\begin{array}{c}\text { Landnutzungskarte } \\
\text { von Aleppo }\end{array}$ & $1: 200.000$ & 2003 & (Landwirtschaftsminister.) \\
\hline
\end{tabular}


Die topographischen Karten im Maßstab 1:200.000, die zwischen 1940-1941 in der Abteilung Kriegskarten und Vermessungswesen im Auftrag vom Generalstab der deutschen Wehrmacht hergestellt wurden, wurden georeferenziert und als Rasterdaten in der Datenbank gespeichert. Die Grundkartenwerke für diese Karten waren die französischen topographischen Karten von 1920 mit dem projizierten Koordinatensystem Syrian Lambert (Deir ez Zor) mit dem Referenzellipsoid Clarke 1880. Die aktuellsten topographischen, arabisch beschrifteten Karten Syriens, wurden zwischen 1972 und 1984 vom syrischen militärischen Vermessungsamt hergestellt. Diese Karten stammen aus den russischen Karten, die auf Basis von Luftbildern 1963 erstellt wurden, und haben die Gauß-Krüger Projektion mit dem geometrischen Datum Pulkovo 1942. Diese Karten wurden von dem Originalpapier eingescannt und georeferenziert bzw. als Rasterdaten gespeichert. Die topographischen Karten wurden für die Geokodierung von den Satellitenbildern, die Entwässerungsnetzbeschreibung und die Unterstützung der morphologischen Auswertung der Satellitenbilder und des digitalen Höhenmodells benutzt. Für die Beschreibung der geologischen Aspekte (die Lithologie, das Alter und die Struktur) in der geomorphologischen Datenbank wurden die geologischen Karten im Maßstab 1:200.000 ebenfalls vom originalen Papier eingescannt und als georeferenzierte Rasterdaten gespeichert (siehe Anhang 1 und 2). Diese Karten wurden 1963 von demselben russischen Team bearbeitet, das die o. g. topographischen Karten erstellt hat. Ein Vergleich zwischen den optimierten Landsatdaten und den geologischen Karten ergibt das folgende Ergebnis: die verschiedenen lithologischen Einheiten sind viel genauer auf Basis der Satellitenbilder als auf der geologischen Karten 1:200.000 zu identifizieren (Abb. 4-1). Deswegen war bei der geomorphologischen Auswertung eine Kombination der beiden Daten in der Datenbank äußerst notwendig.

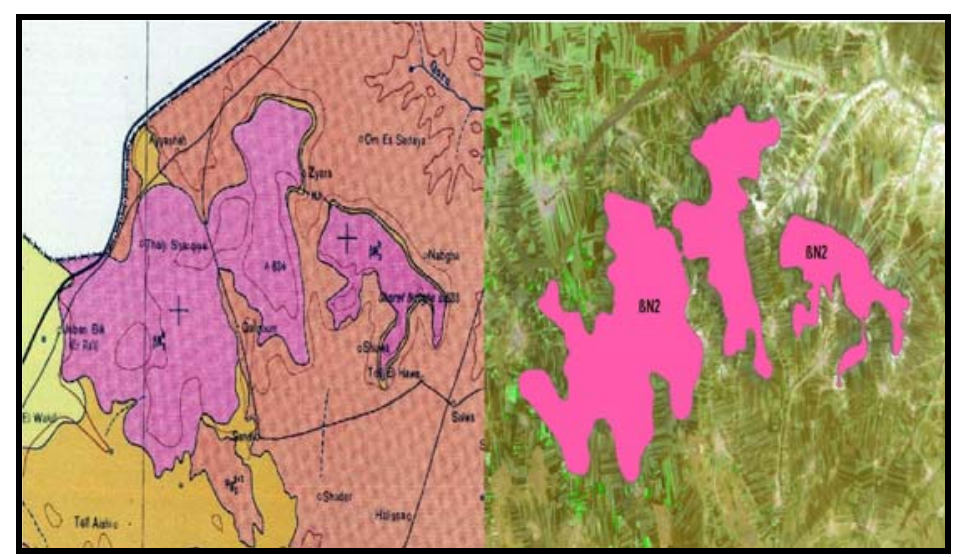

Abb. 4-1: Ermittlung der Lithologie in Landsatbilder (Rechts) im Vergleich zu geologischen Karten 1:200.000 (Links) 


\subsection{Das digitale Höhenmodell}

Neben den oben genannten topographischen Karten standen ferner digitale Höhendaten von SRTM zur Verfügung. Diese Daten sind in Form des Rasterformats gespeichert bzw. bearbeitet und besitzen eine geometrische Auflösung von 90m.

Die SRTM-Daten, die im Internet für das Gebiet freigeschaltet sind, waren bis zum 21.07.2005 noch in einem vorläufigen Bearbeitungszustand. In den alten Daten existieren einige Datenlücken. Mehrere Methoden und Interpolationsverfahren für das Ausfüllen derartiger Lücken wurden in der Literatur (OLAYA, 2004) beschrieben. Zum Beginn der Arbeit waren für das betrachtete Gebiet die alten SRTM-Daten (Version-1) vorhanden. In diesen Daten kommen wenige Lücken vor, was auf die Homogenität des Gebiets deutet. Das Programm SAGA wurde eingesetzt, um die Lücken zu schließen (Abb. 4-2). Ein lückenloses Modell wurde von mehreren Kacheln erzeugt (Tab. 4-2). Das Koordinatensystem des angefertigten Modells ist auf UTM mit dem geometrischen Datum WGS84 umgestellt.

Tab. 4-2: Die verwendeten SRTM-Daten

\begin{tabular}{|c|c|}
\hline Breite & Länge \\
\hline N35 & E036, E037 und E038 \\
\hline N36 & E036, E037 und E038 \\
\hline
\end{tabular}

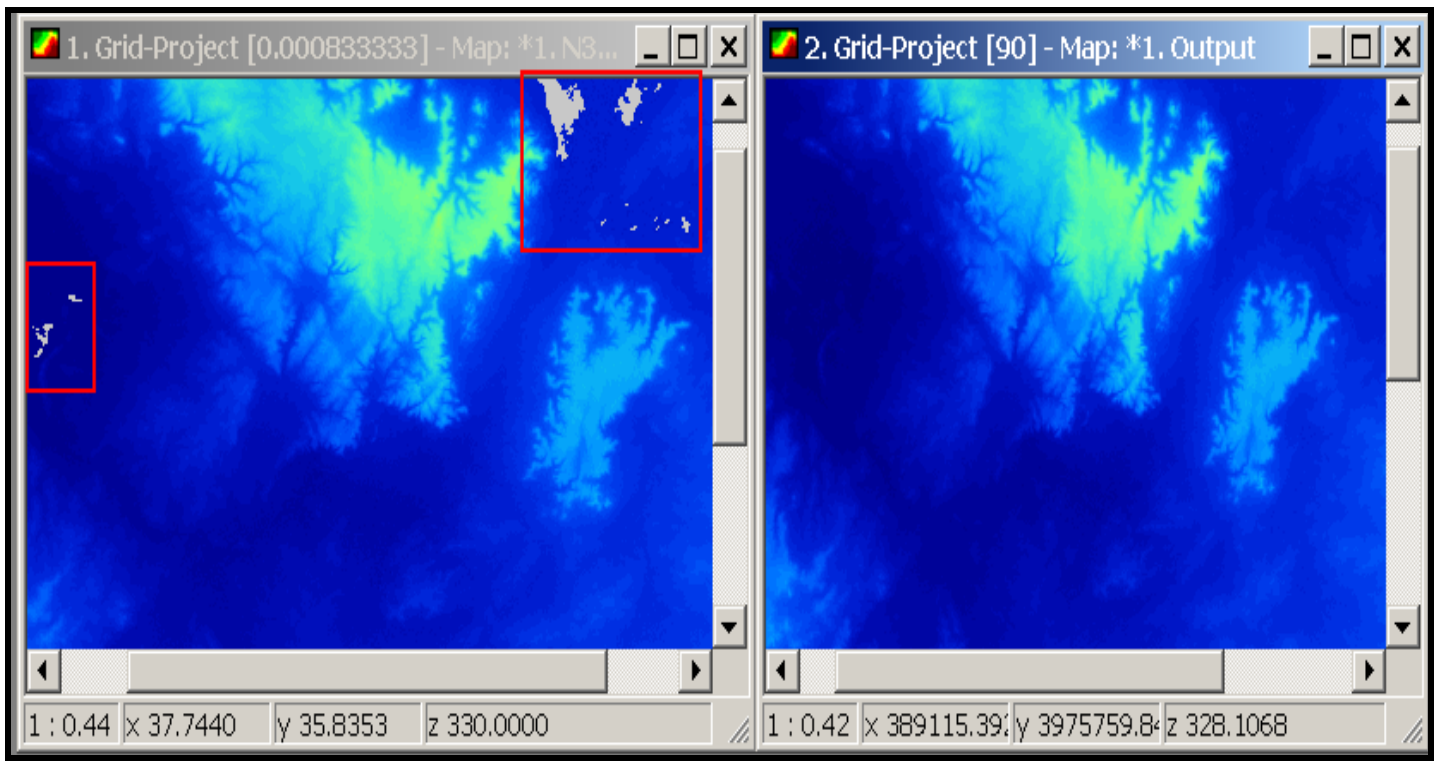

Abb. 4-2: SRTM Rohdaten (Links) und die Daten nach Interpolation (Rechts). 
Die SRTM-Daten wie auch alle Radardaten weisen trotz der Homogenität von Flächen eine körnige Struktur auf. Dieser sogenannte Speckle entsteht durch Interferenzen, die bei der Rückstreuung der kohärenten Mikrowellen an den Objekten der Auflösungszelle entstehen (HILDEBRANDT, 1996; HAGG, 1998), d. h. es handelt sich hier um eine systemimmanente Erscheinung, die durch die Arbeitsweise eines Radarsystems entsteht. Um eine Reduzierung des Speckle zu erreichen, wurde der multidirektionale Lee-Filter im SAGA-Program mit einem 3*3 Kernel verwendet. Mit der Verwendung dieses Filters können die linearen Strukturen wie Täler (Abb. 4-3), Kanten und Hangneigung beibehalten werden (LEE, 1980; OLAYA, 2004).

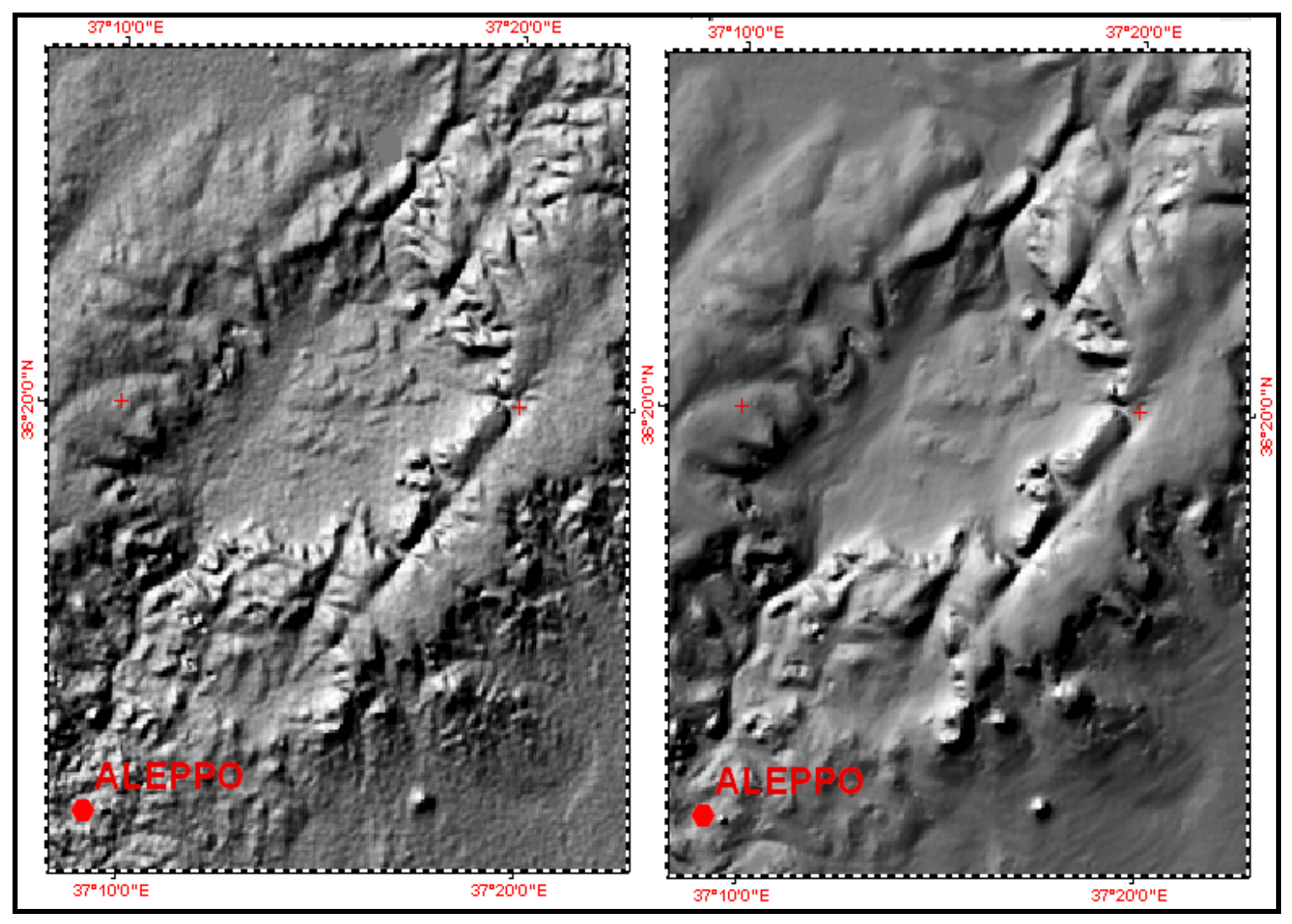

Abb. 4-3: Das geschummerte Reliefmodel der Rohdaten (Links) und nach der LeeFilterung (Rechts) 
Das erzeugte digitale Höhenmodell wurde verwendet, um das Entwässerungsnetz sowie die Einzugsgebiete mittels der GIS-Extension ArcHydro zu extrahieren. Allerdings ergab das Modell bei der ersten Anwendung des ArcHydro Moduls hinsichtlich der Entwässerungsnetzextraktion einen übermäßigen Fehler. Das Arc-Hydro konnte keine Fließrichtung (Flow Direction) im Zentrum des Gebiets erfassen (Abb. 4-4). Der Grund ist, dass die spezifischen Algorithmen in flachen Bereichen die Fließwege nicht erzeugen können, insbesondere, wenn die relativen Höhen gering sind, was der Fall im betrachteten Gebiet ist. Um das Problem zu beseitigen, wurde der Algorithmus von Jenson \& Domingue (1988) verwendet. Mittels dieses Algorithmus können sehr kleine Justierungen in flachen Bereichen (d.h. Zellen, in denen die niedrigsten Nachbarn die gleiche Höhe haben) erreicht werden. Diese Funktion wird in dem Software-Paket TAS (Terrain Analysis System) von der Universität Manchester angeboten (LINDSAY, 2005).

Dieser Fehler war bei den ersten nicht korrigierten, freigeschalteten SRTM-Daten (Version-1) vorhanden. Bei Verwendung von den angefertigten Daten (Version-2), die am 21.07.2005 von der NASA freigeschaltet wurden, wurde derselbe Fehler beobachtet. Deswegen wurde auf die Verwendung der neueren SRTM-Daten verzichtet.

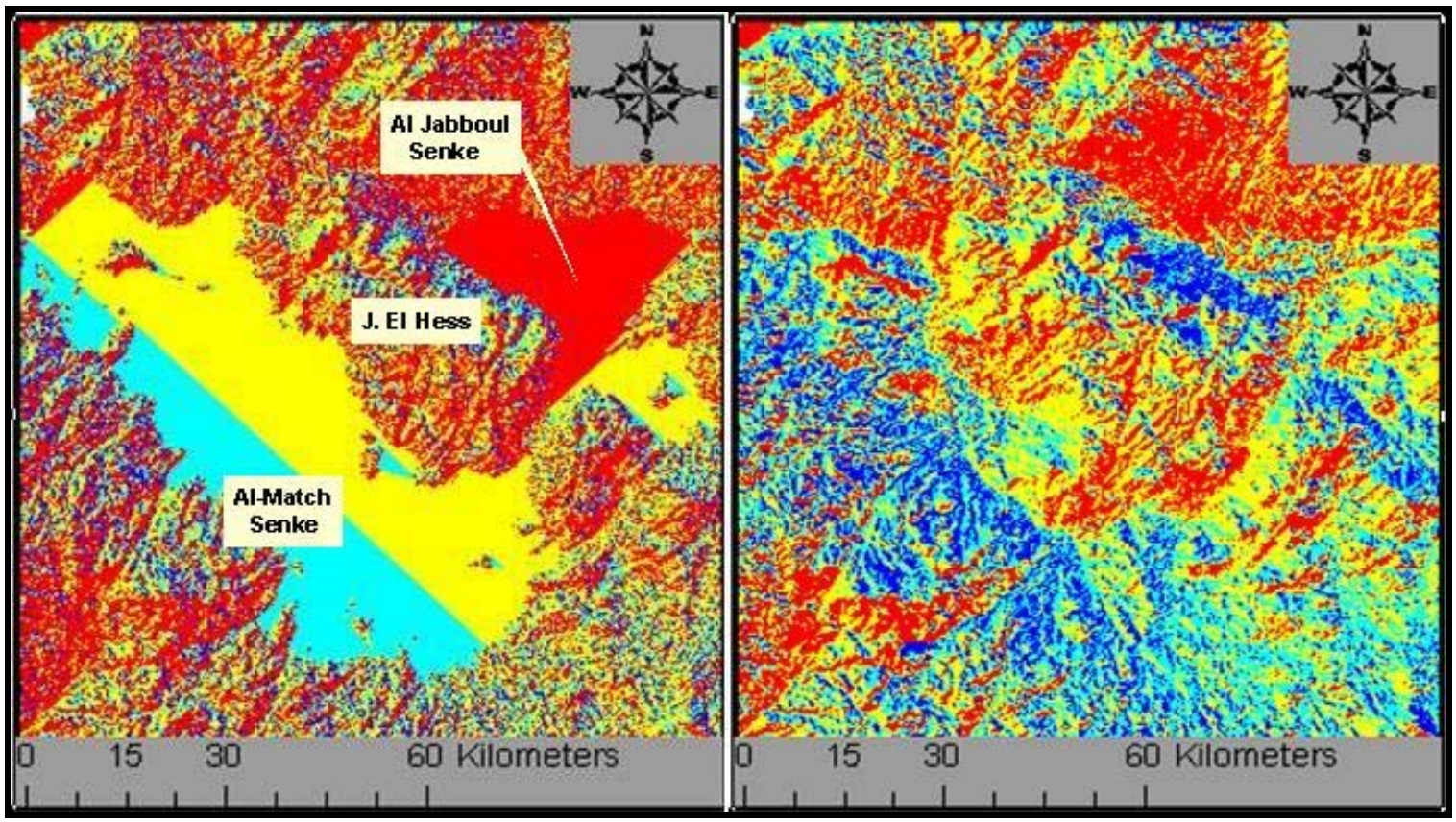

Abb. 4-4: Fehler der Fließrichtungsdefinition in den Rohdaten 


\subsection{Die Satellitendaten}

Für die Studie standen mehrere Satellitendaten (Landsat und ASTER) von verschiedenen Aufnahmezeitpunkten zur Verfügung. Auf Grund der großen Erstreckung ist die Erfassung des Untersuchungsgebietes nur mit vier Landsatszenen möglich (Abb. 2-1). Der größte Bereich (70\% des Gebiets) ist durch die Szenen 173035 und 174035 abgedeckt. Die Szene 173036 erfasst nur ca. 20\% der Fläche. Die Restfläche (10\%) befindet sich auf der Szene 174036 (Tab. 4-3).

Tab. 4-3: Tabelle der verwendeten Satellitendaten

\begin{tabular}{|c|c|c|c|}
\hline Path-Raw & TM-Datum & ETM-Datum & ASTER \\
\hline $173-35$ & 30.07 .1990 & 18.08 .2000 & \\
\cline { 1 - 3 } $173-36$ & 12.06 .1990 & 18.08 .2000 & \\
\hline $174-35$ & 01.10 .1987 & 22.06 .2000 & \\
\hline $174-36$ & 26.05 .1987 & 22.06 .2000 & \\
\hline $173-35$ & - & - & 05.10 .2003 \\
\hline $173-36$ & - & - & 05.10 .2003 \\
\hline $173-36$ & - & - & 25.03 .2005 \\
\hline
\end{tabular}

Die Satellitenrohdaten können erst nach der digitalen Bildverarbeitung zum Einsatz gebracht werden. Die Bildverarbeitung zielt auf die Differenzierung jener Geländeobjekte und Geländegegebenheiten in den Bildvorlagen sowie der kontrastreichen und lagegenauen Wiedergabe ab, wodurch die Interpretation der Satellitendaten viel rationeller und vertrauenswürdiger sein kann. Unter der digitalen Bildverarbeitung versteht man alle rechnergestützten Verfahren der Bearbeitung, Verbesserung und Auswertung von digitalen Bilddaten. Das Prinzip der digitalen Bildverarbeitung beruht darauf, dass die Werte der einzelnen Bildelemente (Pixel) über mathematische Gleichungen in neue Bildelemente überführt werden, welche als Transformationsfunktionen bezeichnet werden.

Die gebräuchlichsten Verfahren sind die geometrischen und radiometrischen Korrekturen (Bildvorbearbeitung), Bildverbesserungen und Klassifikationen. Da es sich in dieser Arbeit um die geomorphologische morphostrukturelle Erfassung geht, wobei die visuelle Auswertung von gewissenhaft aufbereiteten Bildprodukten besser geeignet ist als die digitale Klassifizierung (LIST et al., 1990), wurden hier Bildvorbearbeitung und Bildverbesserungen viel mehr betont als die aufwendige digitale Klassifizierung. 


\subsubsection{Bildvorbearbeitung (Pre-Processing)}

Die Rohdaten der Satellitenbilder haben systematische und nichtsystematische Fehler, welche mittels mehrerer Verfahren korrigiert werden können. Die Daten sind erst nach der Durchführung der Bildkorrektor praktisch einsetzbar sein.

\subsubsection{Geometrische Korrektur}

Die Satellitendaten als digitale Rasterbilder weisen eine spezifische Bildmatrix auf, die aus quadratischen, durch einen Grauwert gekennzeichneten Bildelementen bestehen. Diese Bildelemente werden in einem orthogonalen Koordinatensystem dargestellt, dessen Nullpunkt in der linken oberen Ecke liegt. Diesem zu Folge werden die Pixel in einem Satellitensystem ohne geographische Zuordnung zu einer Kartendarstellung aufgenommen.

Die Korrektur des o. g. nichtsystematischen Fehlers durch eine Georeferenzierung ist unerlässlich, um die Bildkoordinaten in geographische Landeskoordinaten zu transformieren und zu projizieren, welche die Grundvoraussetzung für alle geometrischen Operationen in einem GIS ist. Eine solche Entzerrung führt zu einer problematischen Zuordnung der Grauwerte mit dem jeweiligen Pixel, da die Bildelemente des korrigierten Ausgabebildes sich nicht deckungsgleich mit den Bildelementen des Eingabebildes überlagern lassen (Abb. 4-5).

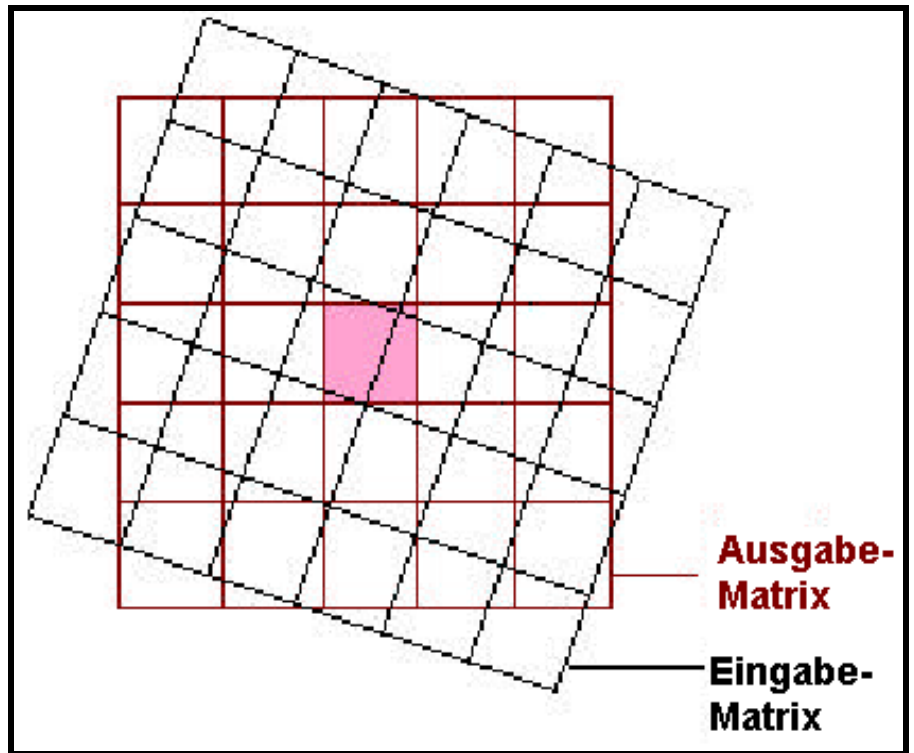

Abb. 4-5: Geometrische Ausgabe-Matrix überlagert von Pixeln in unkorrigierter Lage (LILLESAND \& KIEFER, 2000) 
Das oben dargestellte Problem kann durch den Arbeitsschritt des „Resamplings“ aufgelöst werden. Insgesamt stehen drei verschiedene Methoden für diesen Bildbearbeitungsprozess zur Verfügung, die die Feinheiten der Objektstrukturen mehr oder weniger gut wiedergeben. Die rechnerisch einfachste Methode ist das Verfahren der nächsten Nachbarschaft („nearest neighbour"), bei der jeweils der Grauwert des Pixels aus dem Eingabebild übernommen wird, das dem transformierten Pixel am nächsten liegt. Die anderen zwei Methoden sind die bilineare und die bikubische Interpolation. Sie rufen beachtliche Grauwerteänderungen hervor. Deswegen wurde die o. g. „nearest neighbour“ Methode verwendet.

Die Georeferenzierung der Satellitenbilddaten erfolgte mit Hilfe des Programms ENVI (Environment for Visualizing Images), als „Image to Image“, womit die bereits georeferenzierten Landsatdaten als Basis verwendet wurden. Die Landsatdaten basieren auf dem UTM-Koordinatensystem (Universal Transversal Mercator) mit dem Kartendatum World Geodätischen System 1984, welches in allen neuen Karten Syriens verwendet wird. Das Untersuchungsgebiet, ebenso wie die vier verwendeten Satellitenszenen, liegt in der UTM-Zone $37 \mathrm{~N}$, welche von Vorteil für diese Arbeit ist, da man geometrisch keine Schwierigkeiten wegen des Wandelns zwischen zwei UTM Zonen eingehen muss.

Die ASTER Daten wurden in Level 1A ausgeliefert, d. h. ohne jegliche Korrekturen. Obwohl das Programm von ENVI diese Daten automatisch geometrisch korrigieren kann, wurden diese Daten auch als „Image to Image“ auf Basis der Landsatdaten georeferenziert, um eine qualitativ hochwertige Überdeckung zwischen allen Daten zu schaffen.

\subsubsection{Atmosphärische Korrektur}

Die Absorptions- und Streuprozesse in der Atmosphäre beeinträchtigen den Transport der Solarstrahlung entlang ihres Weges von der Sonne zur Erdoberfläche und von der Erdoberfläche zum Sensor. Je nach Wellenlänge und Zusammensetzung der Atmosphäre wird das am Sensor gemessene Signal verändert. Generell wird Strahlung kürzerer Wellenlängen stärker gestreut als langwellige Strahlung. Eine besonders starke Streuung erfolgt daher im ultravioletten und blauen Anteil des Sonnenlichtes. Deswegen wird die Qualität der Analyse der Erdoberfläche sowie ihrer Prozesse mit Satellitendaten durch Kenntnis um den Zustand der Atmosphäre zum jeweiligen Aufnahmezeitpunkt bestimmt. 
Für eine absolute Atmosphärenkorrektur müssen die verschiedensten atmosphärischen Parameter wie Temperatur, Luftdruck, -feuchte, Aerosolkonzentrationen, etc. zum Aufnahmezeitpunkt flächendeckend und in hoher Dichte vorhanden sein. Für die meisten vorhandenen Szenen für das Untersuchungsgebiet sind diese Daten jedoch nicht erhältlich. Das Untersuchungsgebiet hat klimatisch einen ariden Charakter, womit eine einfache Bildkorrektur (Dunstkorrektur) durchgeführt werden kann, um den Einfluss atmosphärischer Bedingungen zu eliminieren (CHAVEZ, 1989).

Die Methode der Dunstkorrektur basiert auf der Annahme, dass sich im Bild dunkle Objekte (z.B. Gewässer, dunkle Schatten) befinden, die so gut wie keine Rückstrahlung besitzen. Dementsprechend geht der Minimumwert im Histogramm dieser Objekte gegen Null. Ist dies nicht der Fall, so wird der Versatz, der sich in einem Histogramm ergibt, durch die atmosphärische Streuung hervorgerufen. Infolgedessen beruht die Korrektur des atmosphärischen Einflusses auf der kanalweisen Subtraktion des jeweiligen Minimalwerts vom gesamten Histogramm, so dass das gesamte Histogramm in Richtung Nullpunkt verschoben wird (JENSEN, 1996). Der Nachteil der angewandten Methode besteht darin, dass die vereinfachte Annahme, dunkle Objekte verfügen über keine Reflektanz, nicht vollständig mit der Realität übereinstimmt. Zudem berücksichtigt die Methode nur die additive Komponente der atmosphärischen Streuung. Die multiplikative Komponente (transmittance) bleibt unberücksichtigt.

\subsubsection{Bildverarbeitung}

Thematische und physikalische Interpretationen aus den von Satellitensystemen aufgenommenen Reflexions- und Textur-Parameter-Daten lassen Rückschlüsse auf die physikalische und morphologische Beschaffenheit der Objekte in einem Gebiet zu. Die Werte der Reflexions- und Textur-Parameter können mit zielgerichteten verschiedenen Bildverarbeitungsverfahren hervorgehoben und extrahiert werden. Die Zuordnung von Fernerkundungsdaten kann durch visuelle oder digitale Klassifizierung vorgenommen werden. Bei dem geomorphologischen/geologischen Einsatz wird die visuelle Auswertung der Fernerkundungsdaten angestrebt. Im Folgenden werden einige Bildverarbeitungsverfahren, die in dieser Studie durchgeführt wurden, erklärt. 


\subsubsection{Farbkomposition}

Bei der Verarbeitung von Fernerkundungsdaten, vor allem bei der Aufbereitung von Multispektraldaten, wird das Farbsystem der drei Bänder in verschiedenartiger Weise benutzt. Der Vorteil von Farbdarstellungen liegt in der synoptischen Darstellbarkeit von verschiedenen spektralen Informationen. In der Farbenlehre gibt es verschiedene Farbdarstellungsmöglichkeiten. Die RGB- (Red, Green, Blue) und IHS- (Intensity Hue Saturation) Darstellung sind in der Fernerkundung die gebräuchlichste Verfahren. Bei HABERÄCKER (1996) werden diverse unterschiedliche Farbmodelle dargestellt und ihre Zusammenhänge erläutert. Das menschliche Auge besitzt die größte spektrale Sensibilität für die grünen Farben und die geringste Sensibilität für die Farbtöne des blauen Bereichs (Abb. 4-6). Aus diesem Wissen heraus ergibt sich die Forderung für die RGB Darstellung, dass der Kanal mit der größten Grauwertvarianz der Farbe Grün und der Kanal mit der geringsten Grauwertvarianz der Farbe Blau zugeteilt werden sollte.

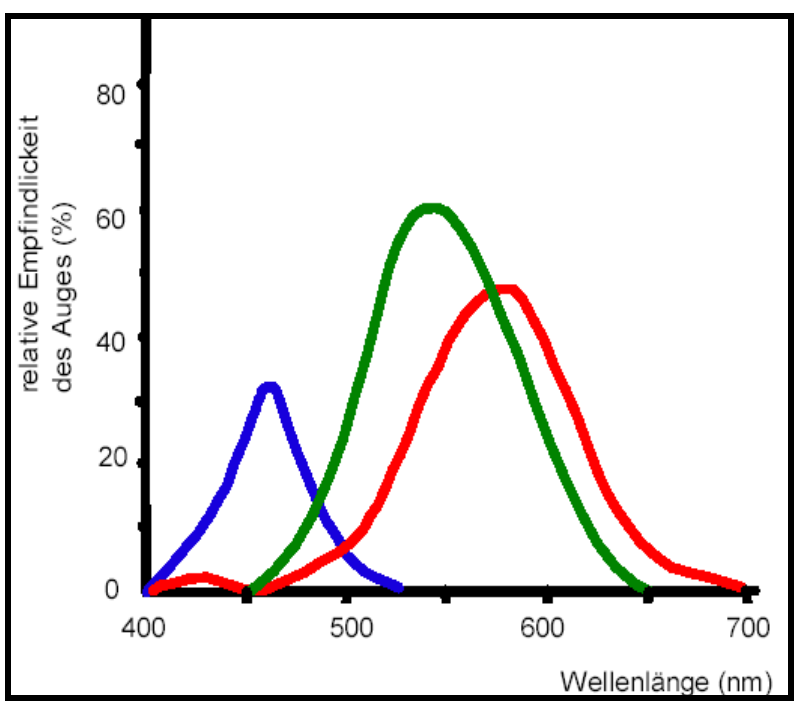

Abb. 4-6: Relative Reaktion der Photopigmente des menschlichen Auges (HABERÄCKER, 1996)

Die Farbdarstellungsmöglichkeiten (RGB Darstellung) von n vorhandenen Bändern ergeben sich wie folgt:

$\mathrm{M}=\mathrm{n} ! /(\mathrm{n}-3) !$

(Gleichung 4-1)

Daher ergibt sich von 6 Bändern der TM-Landsatdaten eine Anzahl von 120 Kanalkombinationsmöglichkeiten. Aufgrund der unterschiedlich starken Korrelation der einzelnen Kanäle untereinander hat jede Kombination der Kanäle einen anderen Informationsge- 
halt. Der höchste Informationsgehalt wird erreicht, wenn die am geringsten korrelierten Kanäle verwendet werden. Farbkompositen aus kaum miteinander korrelierten Kanälen zeichnen sich durch einen hohen Kontrast aus.

Die RGB Farbdarstellung wird im Fachbereich der Fernerkundung Falschfarbendarstellung (Falce Color Composite) genannt, da die dargestellten Daten meist nichts mit den natürlichen Farben des visuellen Spektrumsbereichs zu tun haben. Diese Falschfarbendarstellung (FCC) dient damit gewissermaßen nur als „Übersetzung“ der Bilddaten in eine für das Auge erfassbare Farbe. Die Farbmischbilder müssen nicht zwingendermaßen aus den Rohdaten erstellt werden. Eine Vorverarbeitung durch Ratiobildung, Hauptkomponententransformation oder Grauwertmanipulation ist sogar teilweise ratsam, um eine kontrastreiche Wiedergabe spezieller, gewollter Informationen zu erreichen und die Erkennbarkeit der Gegebenheiten zu verbessern.

Das zweite wichtige Farbmodell ist der IHS (Intensity Hue Saturation) Farbraum, der auch als HSB (Hue, Saturation, Brightness) bekannt ist. In ihm wird Farbe nicht als Mischung aus drei Grundfarben wie bei RGB Darstellungen definiert, sondern durch den Farbton (Hue) als vordefinierten Wert, in Kombination mit einem Wert für die Sättigung (Saturation) und einem weiteren für die Helligkeit (Intensity). Die Sättigung beschreibt dabei den Anteil von Grau im Farbgemisch. Sättigung mit einem Wert von Null bedeutet, dass gar keine Farbe sondern nur ein Grauton dargestellt wird. Die Helligkeit beschreibt die Lage des Farbtons auf einer Skala von Weiß bis Schwarz zusätzlich zu der durch die Sättigung bestimmten Chromatizität. Dieses Farbmodell ist für weitergehende Bildverarbeitungsschritte wie Bildfusion von großer Bedeutung.

\subsubsection{Bildfusion}

Um Informationsgehalte zweier komplementärer Bilddaten in einem neuen Datensatz zu vereinigen, existieren in der Bildverarbeitung verschiedene Kombinationsmethoden wie IHS- und Hauptkomponenten-Transformation (CHAVEZ, 1991). Die Bildfusion von Datensätzen kann vor allem bei Bilddaten mit unterschiedlicher spektraler bzw. räumlicher Auflösung zu Ergebnissen führen, die für eine geologische/geomorphologische Interpretation hilfreich sind. Häufig werden multispektrale Landsatdaten mit räumlich höher auflösenden Bilddaten optischer Sensoren (z.B. SPOT-PAN oder ETM+-PAN) kombiniert, 
um die Vorzüge beider Sensortypen in einem neuen Datensatz wiederzugeben (KAUfMAnN, 1989; ZuMSPREKEL \& PRINZ, 2000). Voraussetzung für die Anwendung der Bildfusion ist eine möglichst genaue Georeferenzierung beider Bilddaten.

Das verwendete Programm ENVI bietet mehrere Fusionsmethoden (Sharpening) an. In dieser Arbeit wurden neben HSV (Hue, Saturation, Value) oder HSI auch PC (principal components transformation) und CN (Color Normalized) verwendet (Abb. 4-7).

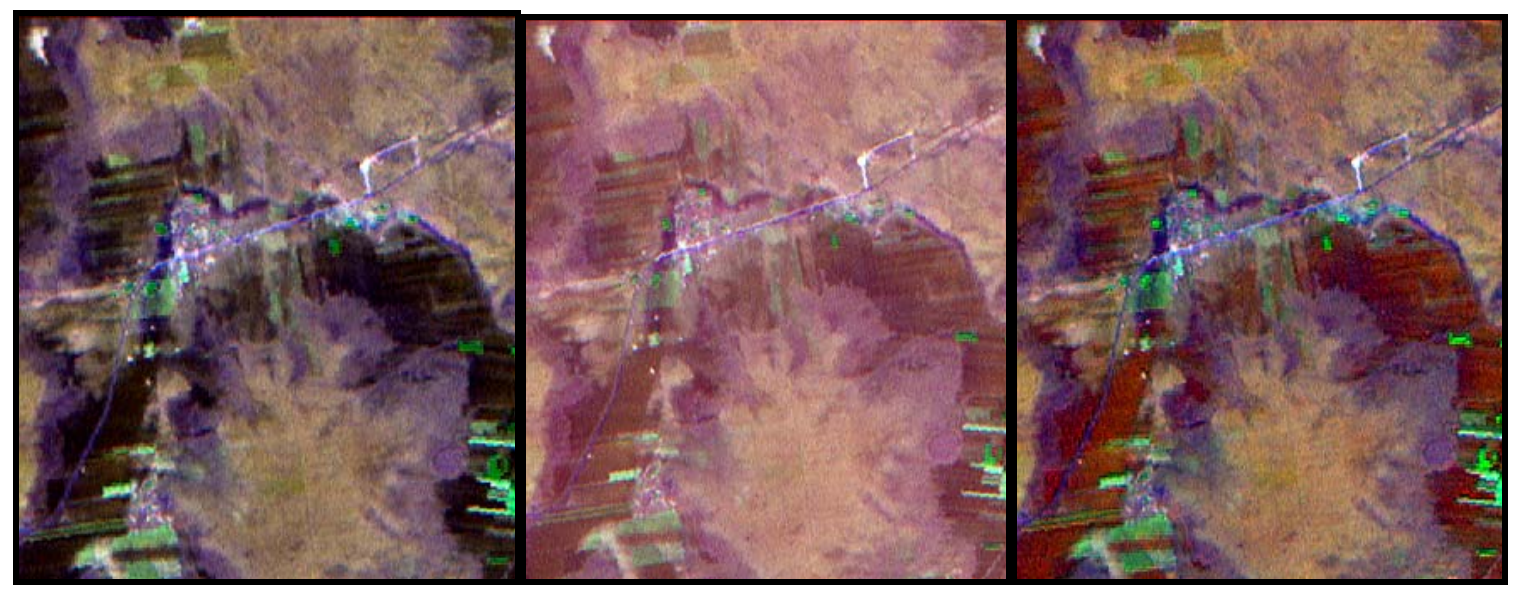

Abb. 4-7: Fusionsmethoden am Beispiel von ETM+ 741 (von Links HSV, PC, CN)

Das Prinzip bei dem Verfahren von IHS (Intensity Hue Saturation) besteht darin, einen Intensity-Layer zu einem vorhandenen RGB-Bild hinzuzufügen und ein höher auflösendes Bild in diesen Layer hinein zu laden (Abb. 4-9). Bei diesem Modell wird die entstandene Intensität (Schwarz-Weiß-Informationen) durch die höher auflösenden panchromatischen Daten ersetzt, und durch die Rücktransformation in den ursprünglichen RGBFarbraum - mit den Parametern Intensität aus Grauwerten sowie Farbton und Sättigung aus den Multispektraldaten - erhält man das transformierte Bild. Dadurch benutzt das IHS-Farbmodell die Größen Intensität (Intensity) von Schwarz bis Weiß, Farbton (Hue) entsprechend der Wellenlänge und Farbsättigung (Saturation) von grau bis Vollton (BÄHR \& VÖGTLE, 1991).

Das PC Verfahren auch PCS (Principal component substitution) basiert auf die Ersetzung eines Bands mit dem Band, das eine höhere Auflösung hat, ohne Spektralenverzerrung zu verursachen. Danach wird eine umgekehrte Transformation durchgeführt. Folglich werden die Multispektraldaten automatisch zur Pixelgröße mit der höchsten Auflösung durch 
die Anwendung von Techniken der nächsten Nachbarschaft, bilinearen oder bikubischen Interpolation transformiert (Abb. 4-8).

Die CN Methode verwendet eine mathematische Kombination eines farbigen Bildes mit Daten der hohen Auflösung. Jedes Band im farbigen Bild wird mit einer Ratio der hohen Auflösungsdaten multipliziert, die durch die Summe der Farbenbänder geteilt werden (Abb. 4-8). In einer geomorphologischen Studie in der Wüste von Saudi Arabien haben AL-JUAIDI et al. (2003) die verschiedenen Fusionsverfahren getestet. Sie fanden heraus, dass das beste Verfahren für die visuelle Auswertung die PCS ist.

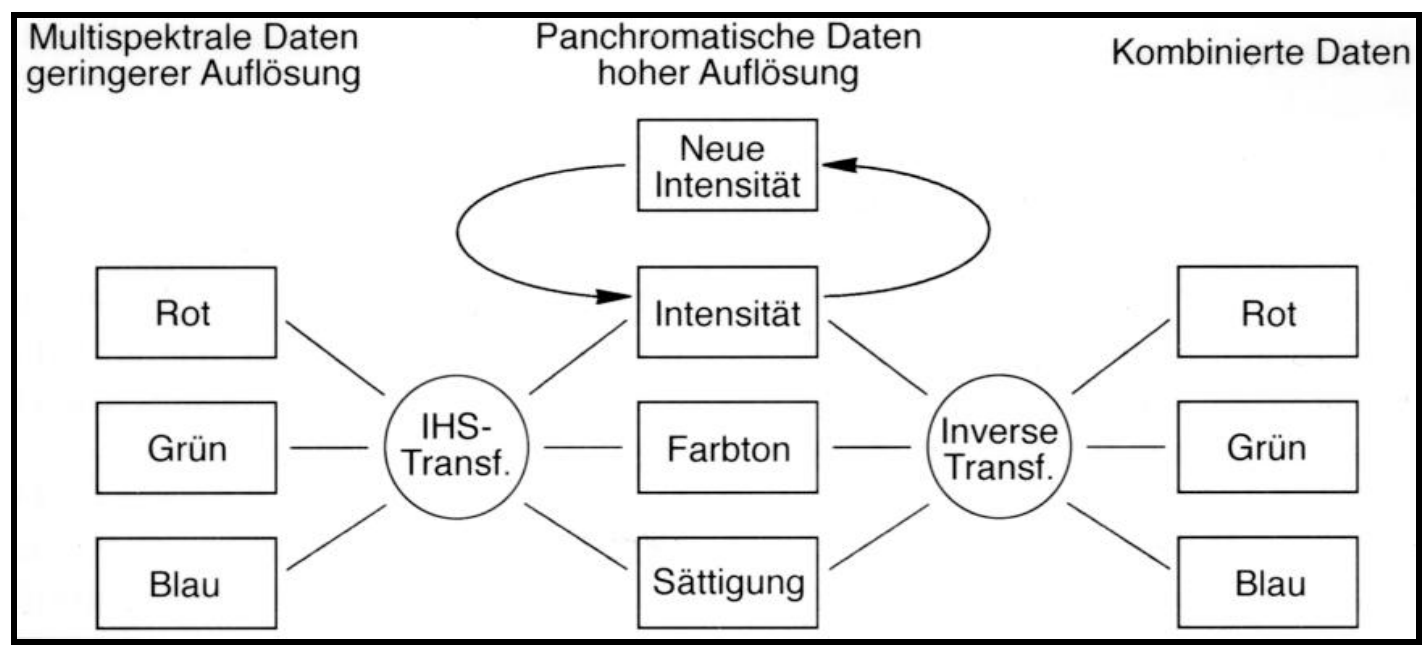

Abb. 4-8: Prinzip der IHS-Transformation (ALBERTZ, 2001) 


\subsubsection{Kontrastverstärkung}

Die Strahlungswerte der Rohdaten häufen sich meist in einem gewissen Bereich der Grauwertskala, womit nur ein geringerer Wertebereich durch die 256 Grauwerte dargestellt wird. Deshalb erscheint ein Bild arm an Kontrast. Um einen hohen Kontrast zu erzeugen, muss die gesamte Breite des zur Verfügung stehenden 8-bit dynamischen Bereichs (256 Grauwerten) genutzt werden. Die Abbildung (4-9) zeigt am Beispiel der Rohdaten der Landsatszene ETM+ 173036 die Grauwertverteilung verschiedener Kanäle.

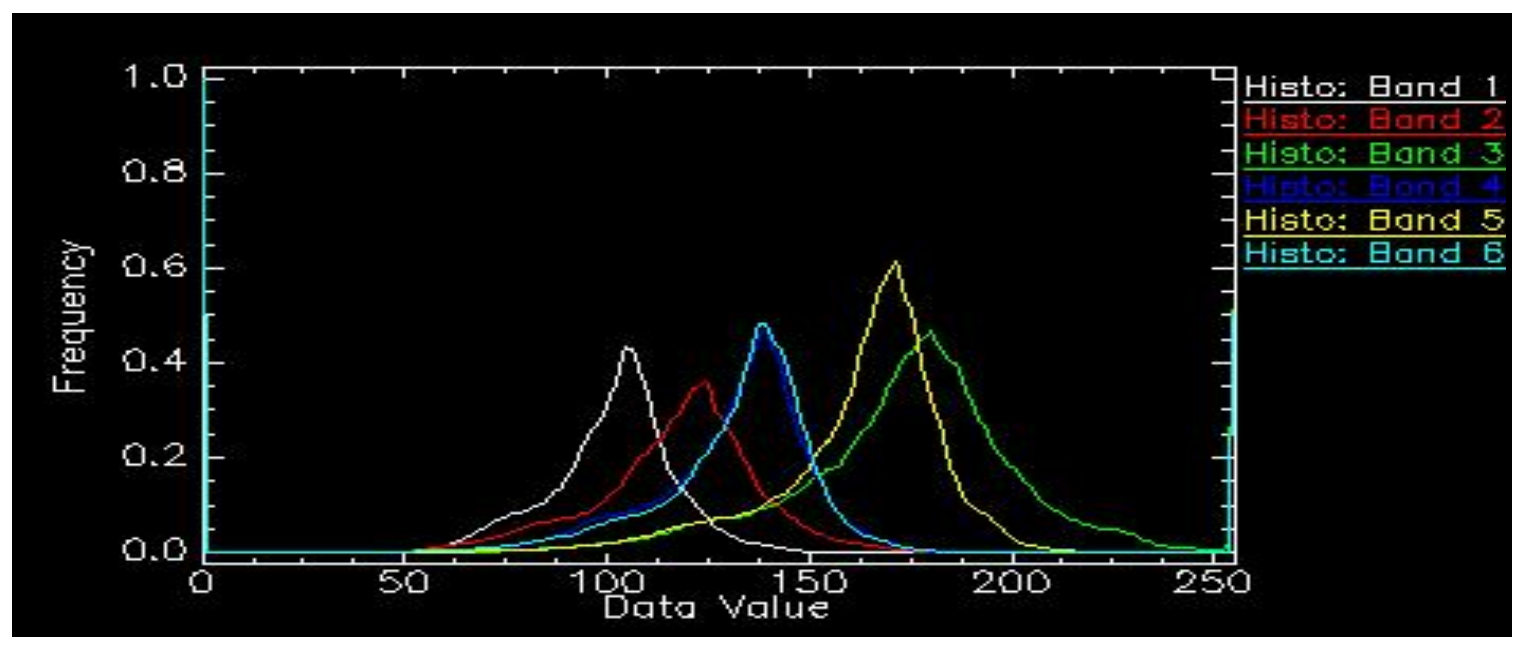

Abb. 4-9: Histogramm der Grauwertverteilung

Das aus diesem Grund kontrastarme und damit schwierig zu interpretierende Bild kann durch eine Kontraststreckung wesentlich verbessert werden (Grauwertstretch). Es gibt mehrere Grauwertstretchverfahren. Für die Interpretation der linearen Strukturen wie Verwerfungen, Schichtstufen und lithologischen Grenzen eignet sich vor allem die Streckung. Die Linear-Streckung dehnt gleichmäßig die Minimal- und Maximalwerte im Bild über dem gesamten vorhandenen Ausgang aus, um die gesamten Unterschiede bezüglich der Graustufen im Bild zu erhöhen (Abb. 4-10).

Die Root-Verbesserung ist auch sehr nützlich. Sie drückt die Strecke der höheren Werte (Helligkeit) zusammen und erweitert die Strecke der niedrigeren Werte (Schwärzung), dadurch können mehr Details in den dunkleren Bereichen eines Bildes (in dem Basaltfelder des Untersuchungsgebiets) unterschieden werden, während ein beliebiges Detail in den helleren Bereichen noch behalten bleibt (Abb. 4-11). 


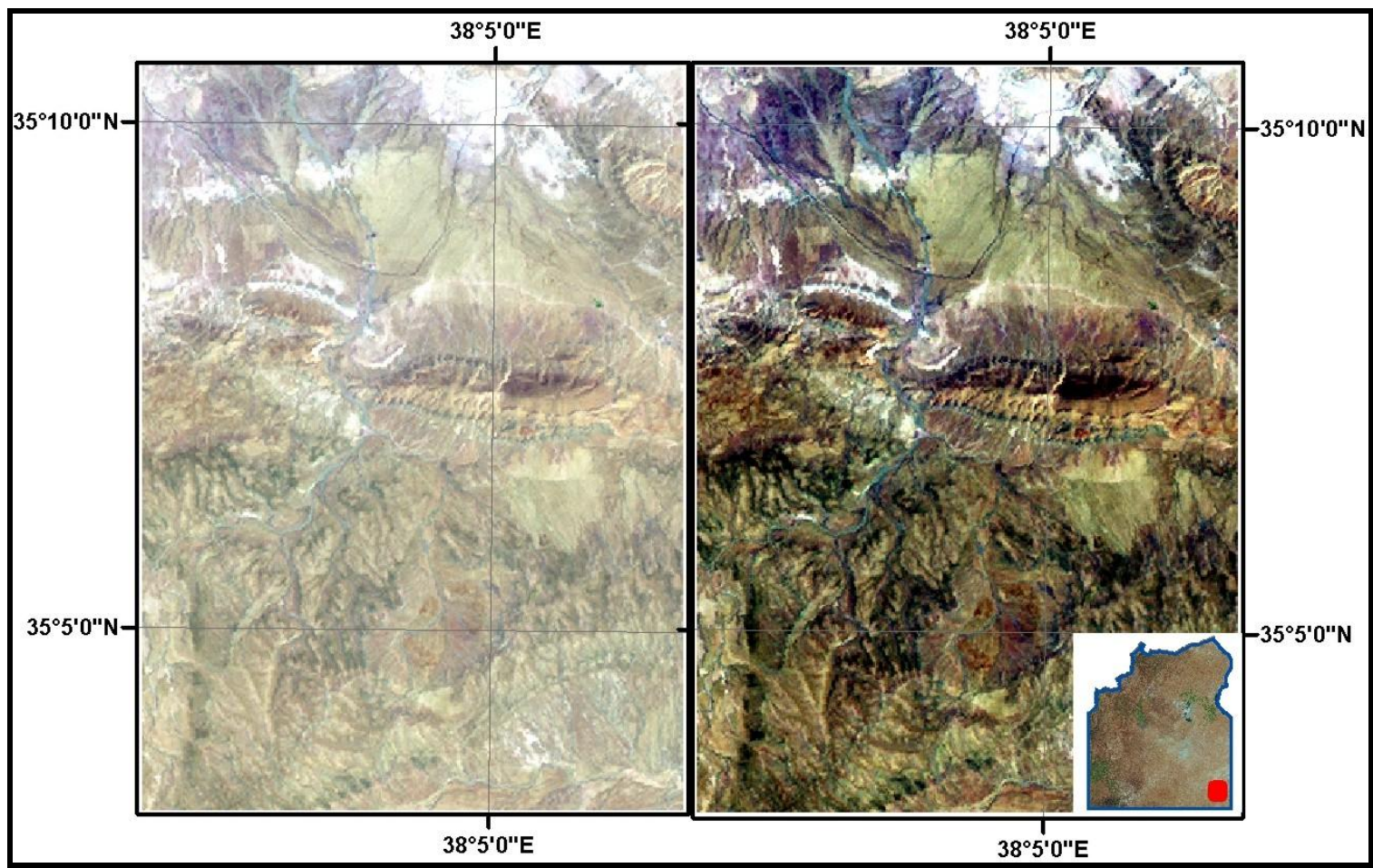

Abb. 4-10: Landsat (741) ohne Erstreckung (Links) und nach der linear (2\%) Erstreckung (Rechts)

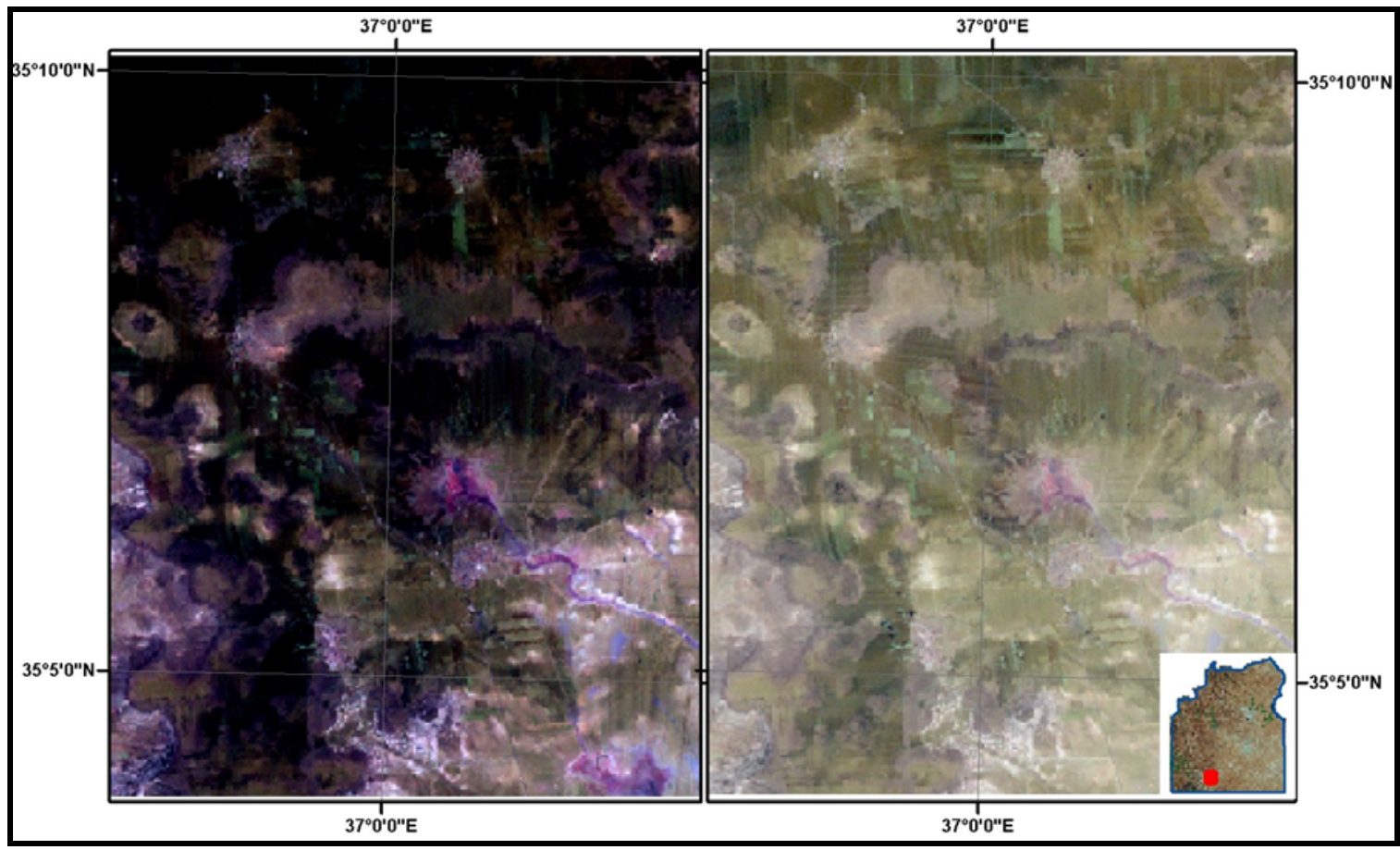

Abb. 4-11: Landsat 731 (Basaltgebiet) links vor der Anwendung des Root-Verfahrens \& rechts nach der Anwendung 


\subsubsection{Hauptkomponententransformation}

Das Verfahren der Hauptachsentransformation (principal component analysis PCA oder Principal Component Transformation PCT) kann zur Bildverbesserung eingesetzt werden und kann gleichzeitig der Datenreduktion dienen (KRONBERG, 1985). Hierbei handelt es sich um eine mathematische Transformation, die neue Bilder erzeugt. Dabei wird eine Berechnung von stochastisch unabhängigen Faktoren durchgeführt, mit denen sich die Datenmatrizen als Linearkombination dieser Faktoren in neue Datenmatrizen (Hauptkomponenten) unter Erhalt der Gesamtvarianz transformieren lassen. Auf diese Weise bilden die neuen erzeugten Bilder ein neues spektrales Koordinatensystem für die Daten (GILLESPIE et al., 1986). Im Gegensatz zu den ursprünglichen Daten weisen sie damit keine linearen Abhängigkeiten mehr voneinander auf. Durch d. o. Transformation wird die Varianz jeder Variablen durch die ermittelten Hauptkomponenten erklärt. Die erste Hauptkomponente erhält den größten Teil der Gesamtvarianz und die zweite Hauptkomponente den größten Teil der Varianz, die von der ersten nicht erklärt wird (da sie orthogonal zur ersten ist) usw. Diese Eigenschaft kann zur Verbesserung von Kanten bei der Auswertung geologischer Strukturen dienen (SANDER, 1996). Die Zahl der Ausgangskanäle ist identisch mit den Eingangskanälen, d. h. bei einer Landsatszene werden somit sechs PCA-Kanäle errechnet. Der erste neue Bildkanal weist den größten Anteil an erklärter Varianz auf, den niedrigsten Anteil im letzten Kanal. Auf diese Eigenschaft bezieht sich die Datenreduktion bei diesem Verfahren, weil ihre Anwendung die Möglichkeit bietet, auf diese Weise eine Satellitenbildszene ohne relevanten Informationsverlust auf weniger Kanäle zu reduzieren. Dieses Verfahren hängt von verschiedenen Faktoren wie Jahreszeit, Landschaftstyp, Bodenbedeckung, gewähltem Ausschnitt usw. ab, welche in Satellitenbildern unterschiedliche Ergebnisse erzielt werden können. Hierbei spielt auch die Materialkorrelation eine große Rolle (SCHOWENGERDT, 1997), z. B. wenn ein Gebiet im Satellitenbild recht homogen von Vegetation bedeckt ist, kommt es durch das ähnliche Spektralverhalten zur räumlichen Korrelation zwischen den Bildpunkten. Bei dem geologischen Einsatz ist der wichtigste Faktor die Gesteinshelligkeit (KRONBERG, 1985), womit einige lithologische Einheiten, die in den Originalbildern unbemerkt bleiben, in dem produzierten Bild sichtbar gemacht werden können. Deswegen eignet sich die HKA für die Differenzierung geologischer Einheiten sehr gut (KENEA, 1997). 
Das ENVI Programm bietet die Möglichkeit unstandardisierte und standardisierte HKA durchzuführen. Diese beruht darauf, dass zwischen den Spektralkanälen typischerweise Unterschiede in der Varianz innerhalb des Kanals erscheinen. Diese Varianzunterschiede machen sich bei der Nutzung einer Kovarianzmatrix (Abb. 4.12) in der unstandardisierten HKA durch eine unterschiedliche Wichtung der Kanäle bemerkbar. Bei der standardisierten HKA werden die eingehenden Spektralkanäle gleichmäßig durch die Nutzung von Korrelationskoeffizienten gewichtet. Allgemein ist die standardisierte HKA unter Verwendung einer Korrelationsmatrix besser als eine unstandardisierte (SINGH \& HARRISON, 1985).

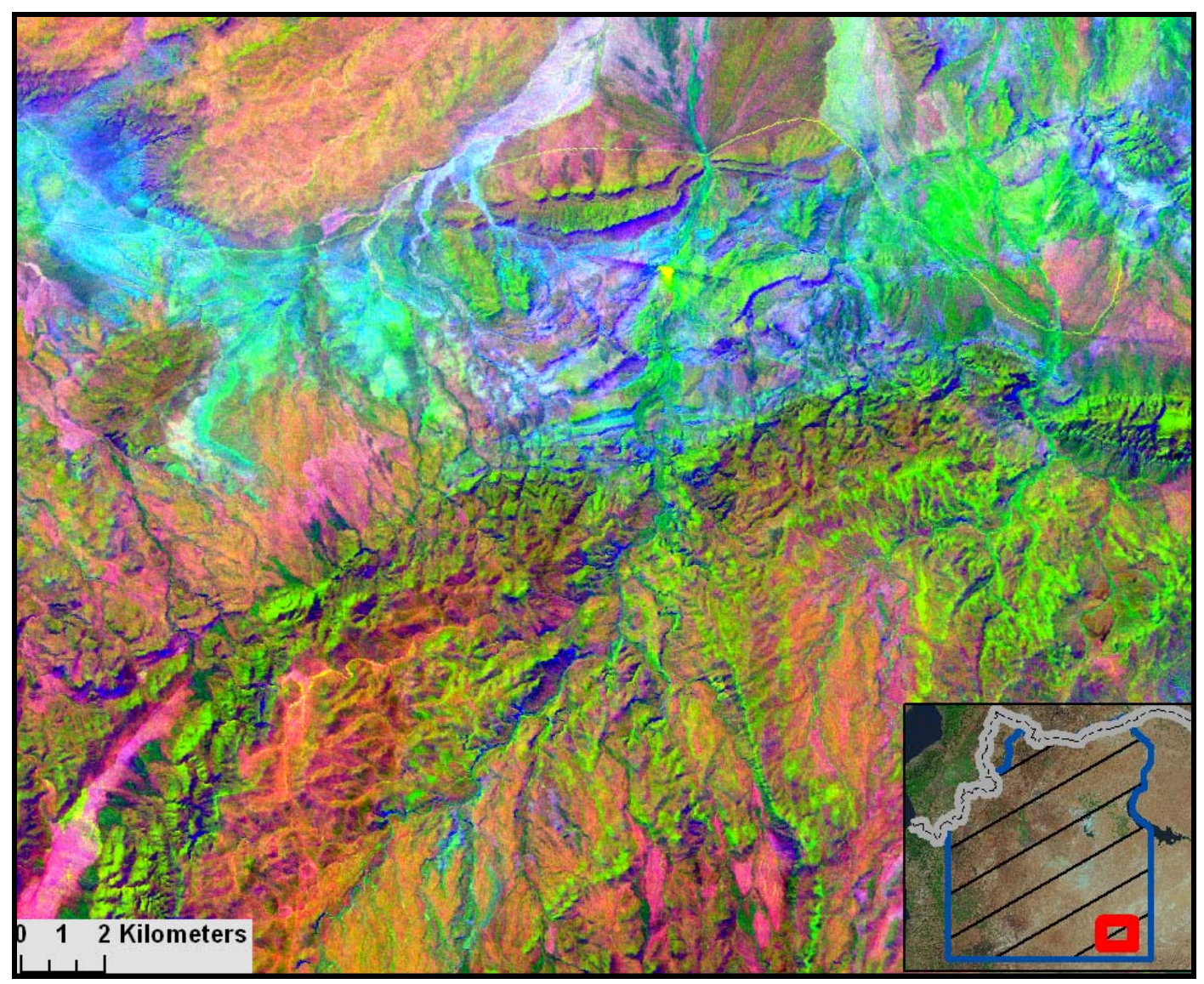

Abb. 4-12: Anwendung des HKA-Verfahrens (Korrelationsmatrix) 


\subsubsection{Quotientenbilder (Ratiobilder)}

Zur Unterscheidung verschiedener Oberflächenmaterialien lassen sich aus Satellitendaten durch rechnergesteuerte Manipulationen Ratioszenen aus verschiedenen Spektralbändern herstellen. Auf diesen Ergebnisbildern können alle redundanten Informationen eliminiert und verdeckte Hinweise hervorgehoben werden. Diese arithmetische Verknüpfung zweier oder mehrerer Kanäle kann zu einer verbesserten Darstellung bestimmter Informationen führen. Sie kann mit einem Datensatz durchgeführt werden, aber auch mit Daten verschiedener Aufnahmezeitpunkte, z. B. für Monitoring-Zwecke kann diese Verknüpfung sinnvoll sein.

In dieser Arbeit wird vorwiegend auf die Division zweier Kanäle eingegangen. Das Ratio-Verfahren kann als reines Reduktionsverfahren gesehen werden. Ratiobilder werden erzeugt, indem die gesamten Intensitäten zweier Spektralkanäle Pixel für Pixel durcheinander dividiert werden. Die Grauwerte des neuen Bildproduktes stellen kein Maß für die Reflexionsintensität dar, sondern das Verhältnis der Helligkeit zweier Kanäle. Die Ratiobildung kann zu einer kontrastreicheren und informativeren Darstellung des Bildinhaltes, bzw. einer bestimmten, den Interpreter interessierenden, Information führen. Durch die Division werden Albedo- und Beleuchtungsunterschiede unterdrückt, deswegen lässt sich eine Verringerung der topographischen Einflüsse erzielen. Allerdings hat diese Verarbeitung vor allem in der Geologie Nachteile, die in einer Verschlechterung des SignalRausch-Verhältnisses liegen. Die erstellten Ratiobilder können in Farbkompositen weiterverarbeitet werden, oder auch als zusätzlicher Inputkanal in eine automatische, rechnergestützte Klassifikation einfließen. Die erfolgreiche Informationsgewinnung mit Hilfe der Ratiobildung hängt zu einem großen Teil von den verwendeten Kanälen ab. Die Kanäle, die zur Ratiobildung ausgesucht werden, sollten so gewählt sein, dass der eine innerhalb und der andere außerhalb der spektralen Region liegt, in der das Zielobjekt ein Minimum oder ein Maximum an Reflexionsintensität besitzt (VINCENT, 1997). Die Wahl der optimalen Kanäle kann mit Hilfe der Signaturanalyse der originalen Bilddaten erfolgen. Die Ratiobilder finden vor allem in der Geologie verbreitete Anwendung, da sie manchmal geringfügige Reflexionseigenschaften von Materialien der Erdoberfläche verstärken können (GoETZ et al., 1975; WiLliAMS, 1983). Durch die Anwendung der Ratioverfahren können bestimmte existierende Absorptionsbanden eines Materials lokalisiert werden, da die Reflexion zweier Wellenlängenbereiche verglichen wird, z. B. Tonminerale können z. B. mit der Ratio TM 5/7 (HILDEBRANDT, 1992) differenziert werden. Die 
meisten Ratio-Bildungen sind meistens für die Flächenabgrenzung nach quantitativen Kriterien gut geeignet, insbesondere um die Information für die visuelle Interpretation hervorzuheben.

\subsubsection{Filtertechniken}

Durch die Anwendung von Filtertechniken können im Grunde genommen erwünschte oder unerwünschte Information getrennt oder erwünschte Information hervorgehoben werden (DE LANGe, 2006). Dabei handelt es sich um lokale Operationen, weil die Werte von Rasterzellen in Abhängigkeit von umgebenden Pixeln verändert werden. Diesen Operationen dienen zahlreiche unterschiedliche Algorithmen, die sich für verschiedene Verarbeitungsziele eignen.

Die Filteroperationen werden oft zur Bildverbesserung eingesetzt. Einige Operationen wie Tiefpassfilter (low pass filter) haben eine Glättung oder Kontrastausgleich zu Folge. Solche Filter können zur Verminderung des Rauschens oder zur Beseitigung lokaler Fehler (Werteninterpolation) verwendet werden. Andere Operationen wie Hochpassfilter (high pass filter) haben den Effekt, dass die hohen Frequenzen in den Bildern hervorgehoben werden. Hochpassfilter dienen unter anderem zur Betonung von Kanten. Diese genannten Filter können unter den Begriff lineare Filter eingestuft werden. Die lineare Filterung kann auch in einer ausgesuchten Richtung eingesetzt werden, welche besonders in der Geologie zur Verfolgung geologischer Strukturen nützlich ist. So entstand der Begriff der morphologischen Filter und Kantendetektoren wie Laplace und Sobel, die die Bereiche starker Grauwertänderungen als Kanten hervorheben. Außerdem gibt es statistische Filter (Median und Gaussian etc.) und adaptive Filter (Frost, Lee), die vor allem das „Rauschen“ entfernen.

Die Filteroperationen basieren auf sich bewegende Filter-Fenster (moving windows), die vom Ursprung der Rastermatrix ausgehend (in der Regel links oben) über die Zellen und in einzelnen Schritten jeweils den Wert des gerade in der Mitte befindlichen Pixels berechnen. Die Größe des Filterfensters (Filtermatrix) beeinflusst die Erkennbarkeit der Strukturen. Die großen Fenster können für die Aufteilung großflächiger Bereiche wie Landnutzung und Geologie verwendet werden. Demgegenüber eignet sich das kleine Fenster eher für die Betonung von Lineamenten (Abb. 4-13). Während die ersten unter dem Problem der geringen Trennschärfe leiden, betonen die zweiten systematisch unerwünschten Informationen, wie zum Beispiel anthropogene Strukturen. 


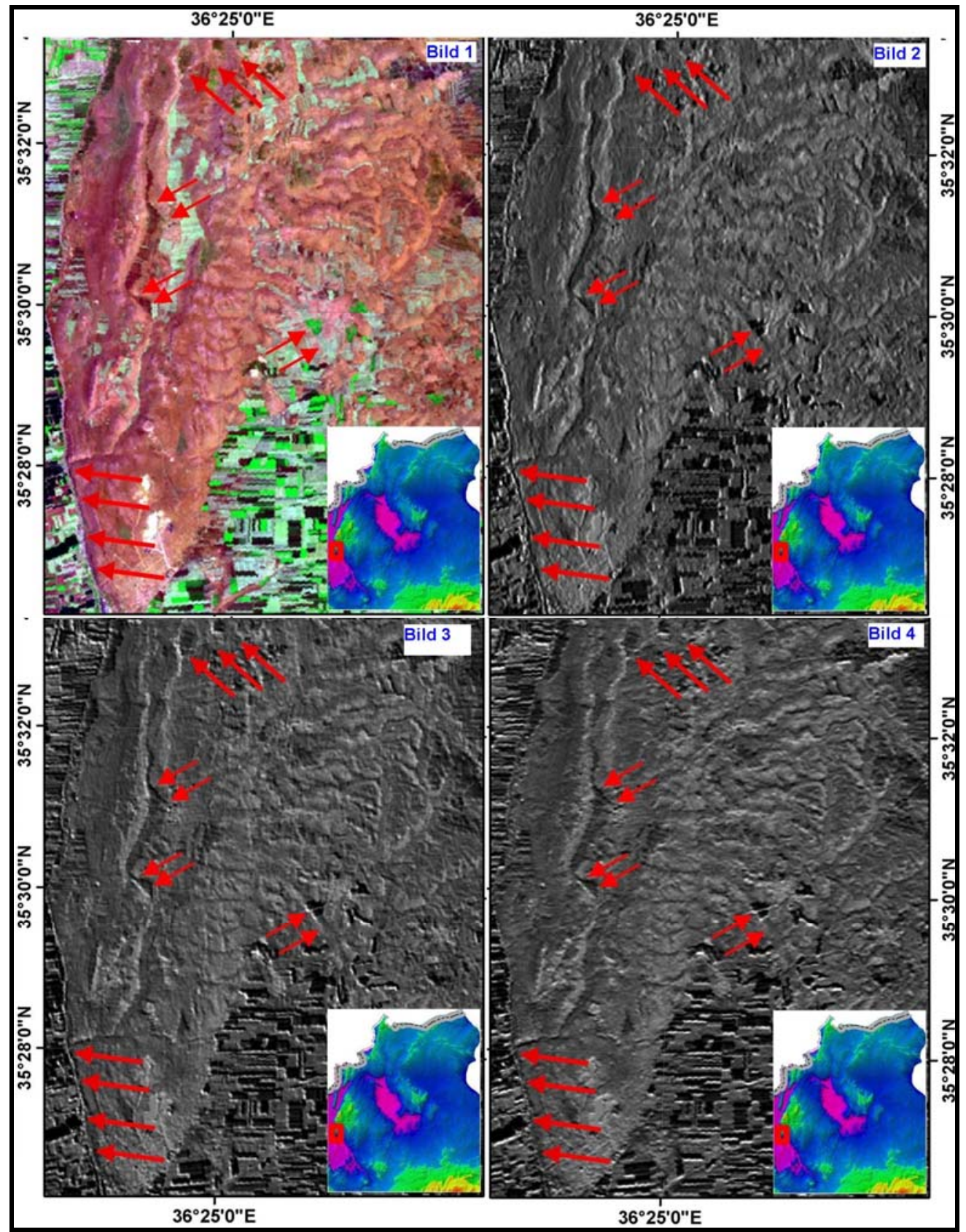

Abb. 4-13: Hervorhebung der linearen Morphostrukturen mittels Richtungsfiltern

- Bild 1: ETM+ Farbkombination (741).

- Bild 2: ETM+ Kanal 7 nach einer Filterung mit 0 Grad und die Betonung von $\pm N$-S streichender Strukturen (Pfeile links unten).

- Bild 3: ETM+ Kanal 7 nach einer Filterung mit 135 Grad und die Betonung von $N W-S E$ streichenden Lineationen (Pfeile in der Mitte).

- Bild 4: ETM+ Kanal 7 nach einer Filterung mit 90 Grad und die Betonung von NE-SW sowie E-W streichenden Lineationen (Pfeile oben). 


\section{Kap it e l 5}

\section{Darstellung der Geomorphologie des Gebiets}




\section{Darstellung der Geomorphologie des Gebiets}

Für die geomorphologische Darstellung bzw. Untersuchung stehen mehrere Methoden zur Verfügung (Tab. 5-1). Durch die Anwendung von einer dieser Methoden kann eine spezifische geomorphologische Karte erstellt werden. Demgegenüber führt die Kombination zweier oder mehrerer dieser Methoden zu einer allgemeinen geomorphologischen Darstellung bzw. Analyse, die eine umfassende Illustration ergeben kann.

Tab. 5-1: Die Methoden der geomorphologischen Untersuchung (DEMEK et al., 1982)

\begin{tabular}{|c|c|}
\hline Analyse & Beschreibung \\
\hline Morphologisch & $\begin{array}{l}\text { Sie schließt auch die morphometrische Analyse ein und besteht aus: } \\
\text { der Festlegung der Grenzen und Dimensionen der einzelnen Relief- } \\
\text { elemente, der Bestimmung der Komponenten der komplexen Relief- } \\
\text { formen, der Ermittlung der Beziehungen zwischen den einzelnen Re- } \\
\text { liefelementen, des Charakters ihrer Vergesellschaftung und des } \\
\text { Arealgefügemusters ihrer Anordnung. }\end{array}$ \\
\hline Morphofaziell & $\begin{array}{l}\text { Es handelt sich um die Beziehung zwischen dem Relief und den ent- } \\
\text { sprechenden Ablagerungen. Sie verlangt Laboruntersuchungen zur } \\
\text { Bestimmung des Alters der Ablagerungen. }\end{array}$ \\
\hline $\begin{array}{l}\text { Morpho- } \\
\text { strukturell }\end{array}$ & $\begin{array}{l}\text { Die Methode befasst sich mit der Beziehung zwischen den Relieffor- } \\
\text { men und der geologischen Strukturen. Eines der wichtigsten Verfahren } \\
\text { dabei ist die Auswertung von Luft- und Satellitenbildaufnahmen. }\end{array}$ \\
\hline $\begin{array}{l}\text { Morphoneotek- } \\
\text { tonisch }\end{array}$ & $\begin{array}{l}\text { Diese Methode ist auf den Zusammenhang der Reliefformen mit jun- } \\
\text { gen Krustenbewegungen gerichtet. Sie sollte stets aus dem Studium } \\
\text { der gesamten geologischen Geschichte des Untersuchungsgebiets aus- } \\
\text { gehen. Sie verlangt die Berücksichtigung der jungen und alten Bewe- } \\
\text { gungen, ihrer Arten, Trends und Intensitäten. }\end{array}$ \\
\hline $\begin{array}{l}\text { Morphoge- } \\
\text { ographisch }\end{array}$ & $\begin{array}{l}\text { Hier geht es um die Beziehung des Reliefs zu anderen Geosphären der } \\
\text { Erde (Atmosphäre, Hydrosphäre, Kryosphäre und Biosphäre). }\end{array}$ \\
\hline $\begin{array}{l}\text { Morphodyna- } \\
\text { misch }\end{array}$ & $\begin{array}{l}\text { Sie beschäftigt sich mit den Erläuterungen der Dynamik der gegenwär- } \\
\text { tigen endogenen und exogenen Vorgänge und verlangt damit die fol- } \\
\text { genden Verfahren: i. Geländeaufnahmen an bestimmten Stellen (geo- } \\
\text { dätische Messungen etc.), ii. Prognosen katastrophenartiger Ereignisse } \\
\text { auf Basis der statistischen Analyse der Intervalle ihres Auftretens und } \\
\text { iii. Experimentelle Untersuchungen und Auswertungen von Luft- und } \\
\text { Satellitenbilder, die vor und nach den bestimmten Ereignissen aufge- } \\
\text { nommen wurden. }\end{array}$ \\
\hline $\begin{array}{l}\text { Paläogeomor- } \\
\text { phologisch }\end{array}$ & $\begin{array}{l}\text { Sie führt zur Erfassung des Reliefs vergangener geologischer Zeiten, } \\
\text { wobei die Analyse des gegenwärtigen Reliefs eine besondere Berück- } \\
\text { sichtigung der Umgestaltungsstadien präexistenter Erdoberflächen in } \\
\text { das gegenwärtige Relief verlangt. }\end{array}$ \\
\hline
\end{tabular}


Die geomorphologische Untersuchung konzentrierte sich während der ersten Hälfte des 20. Jahrhunderts auf die Klassifikation und die Geschichte der Landschaft. Damals wurde die Studie der Geomorphologie auf die regionale Skala der Physiographie benannt (BLAIR, 1986). In der zweiten Hälfte entwickelte sich die Studienrichtung, die sich mit den Prinzipien der Mechanik und der Fließdynamik der spezifischen geomorphologischen Prozesse (fluviale und äolisch etc.) beschäftigt (STRAHLER, 1952). Bis Mitte der 70iger Jahre verwendeten die Geomorphologen verschiedene Typen topographischer Karten, um die Hauptmerkmale der Erdoberfläche zu beschreiben, weil sie Informationen über die Morphometrie und Morphographie des Georeliefs enthielten. Durch die Betrachtung der topographischen Karten können viele Landformen verschiedener Größen unterschieden werden. Doch sie schließen nicht alle wichtigen geomorphologischen Landformen ein, insbesondere diejenigen Formen, die zu klein im Vergleich zu ihrem Maßstab sind. Neben der morphometrischen und morphographischen Anwendung können die topographischen Karten nach Ansicht von KLIMASZEWSKI (1982) für die genetische Analyse wie Interpretation der Landschaftsformen eingesetzt werden, obwohl sie keine Informationen über das Alter und den Ursprung der Landformen enthalten.

Die geomorphologischen Studien wurden mit zunehmender Verfügbarkeit der Fernerkundungsdaten verschiedener Auflösung nicht mehr nur einfach durchgeführt, sondern es entwickelte sich die Tendenz, integrierte Darstellungen zu verwenden, um ein besseres Verständnis von der Geomorphologie zu ermöglichen (BLAIR, 2003). Mittels der Fernerkundung lassen sich vier Arten der geomorphologischen Untersuchungen differenzieren: die morphometrischen, die morphographisch/genetischen, die morphodynamischen und die geomorpho-ökologisch ausgerichteten Untersuchungen (VERSTAPPEN, 1977). Jeder Typ kann sich in bestimmter Weise die Fernerkundung zu Nutze machen. Vor allem dienen die Fernerkundungsdaten auf Grund ihres großen Vorteils, Daten in Makroskalen zu liefern, als Grundlage für die geomorphologische Kartierung. Die Qualität und Verteilung der Daten, die aus der Fernerkundung basieren, sind viel detaillierter als die Daten, die durch traditionelle geomorphologische Beobachtungen und Vermessungen gesammelt werden können. Darüber hinaus ermöglicht der Vergleich der Satellitenbilder aus verschiedenen Jahreszeiten eine Vorstellung über Art und Intensität geomorphologischer Prozesse (Morphodynamik) in großen Gebieten. 


\subsection{Geomorphometrische Analyse des Arbeitsgebiets}

Die Geomorphometrie beschäftigt sich hauptsächlich mit den habituellen und räumlichen Formen des Georeliefs sowie deren Eigenschaften. Sie ist nach DIKAU (1988) als quantitative Geomorphologie zu verstehen. Mit der exakten Definition der geomorphographischen Kennzeichnung des Oberflächenreliefs (ROESCHMANN \& LEHMEIER, 1993) werden die Teilbereiche der Geomorphometrie und Geomorphographie eng zusammengeführt. Die Geomorphographie im engeren Sinn ist die Beschreibung von Erdformen mit qualitativen Methoden. Meist wird auch die Geomorphometrie in die Definition mit einbezogen, so dass Geomorphographie als Beschreibung der Erdformen mit qualitativen und quantitativen Methoden definiert werden kann. Die Charakteristik und quantitative Beschreibung der Landschaftsmerkmale wurde in der Veröffentlichung von PENCK (1894) als Orographie und Orometrie bezeichnet. In seiner Arbeit „Morphologie der Erdoberfläche" hat PENCK (1894) diese Wissenschaft unter den Begriffen Morphographie und Morphologie erläutert. Nach EvANS (1972) lässt sich die Geomorphometrie in Disziplinen der allgemeinen und speziellen Geomorphometrie gliedern (Abb. 5-1). Die allgemeine Geomorphometrie (general geomorphometry) umfasst die Messung und Analyse der Charakteristika des Georeliefs, die auf jede kontinuierliche Oberfläche anwendbar ist. Die spezifische Geomorphometrie (specific geomorphometry) beinhaltet die Messung und Analyse spezifischer Reliefformen, die durch eindeutige Definitionen von räumlich benachbarten Reliefformen abgrenzbar sind. Beispiel hierfür ist die Analyse der Form von Entwässerungsnetzen oder Einzugsgebieten. Die wichtigsten Parameter in der Geomorphometrie nach Evans (1980) sind Höhen und deren Statistik, Hangneigung (Gradient), Exposition und Krümmung (Kurvatur, Wölbung). Andere Parameter wie Höhenverteilung (Reliefenergie, Rauheit) und Hydrologische Maße (Flussdichte, Länge der Abflusspfade) werden auch in bestimmten Fällen herangezogen. Die statistische Darstellung der generellen geomorphometrischen Attribute (EvANS, 1980) wurde verwendet, um das Georelief sowohl auf lokale als auch auf regionale Skala zu kennzeichnen (MARK, 1975). Die Zahlengewinnung für die geomorphometrischen Darstellungen (zahlenmäßige Erfassung) der Landformen wurde zu Beginn, je nach Maßstab der topographischen Karten, entweder durch Messungen im Gelände oder auf der Karte, welche viel Arbeitsaufwand erfordert, durchgeführt. Diese wurde durch den Einsatz von Höhenmodellen, die seit Ende der siebziger Jahre verwendet wurden, vielfach erleichtert. 
Unter der Bezeichnung Digitales Höhenmodell (DHM) Eng. Digital Elevation Model (DEM) versteht man eine vereinfachte digitale Beschreibung des Georeliefs. Das digitale Höhenmodell besteht aus einer Menge dreidimensionaler Koordinaten in gitterförmiger Anordnung. Weniger gebräuchlich ist der Begriff des Digitalen Geländemodells (DGM) Eng. Digital Terrain Modell (DTM). Wobei der Datensatz des DHMs sich auf die Speicherung von Höhenwerten beschränkt, kann das DGM die Repräsentation topologischer sowie semantischer Informationen wie Beschreibung von Geländekanten, Dämmen, Gräben etc. einschließen (WeIBEL \& HeLler, 1991). Das bedeutet im Fall des DGMs, dass einzelnen Punkten eine geländespezifische Bedeutung zugeordnet werden kann, oder dass Punkte miteinander in Beziehung gesetzt werden, um lineare, im Gelände vorkommende Strukturen zu modellieren. Ebenso können für einzelne Punkte auch sekundäre Informationen wie Neigung (Slope) oder Exposition (Aspect) gespeichert sein. Das DHM als dreidimensionales System mit XYZ-Daten ist demzufolge ein Oberflächenmodell mit einer einzigen Z-Koordinate an einem Punkt. Es wird daher häufig als "2.5DDatenmodell" bezeichnet, da eine echte dritte Dimension als Tiefenfunktion nicht vorhanden ist (WeIBEL \& Heller, 1991). Die Anwendung der DGM- oder DHM-Daten hat eine Neuorientierung in der Reliefanalyse zur Folge. Dadurch sind die Disziplinen der Hydrogeomorphologie, der Geomorphometrie (quantitative Geomorphologie) und der Morphostruktur bzw. Morphotektonik bedeutend entwickelt.

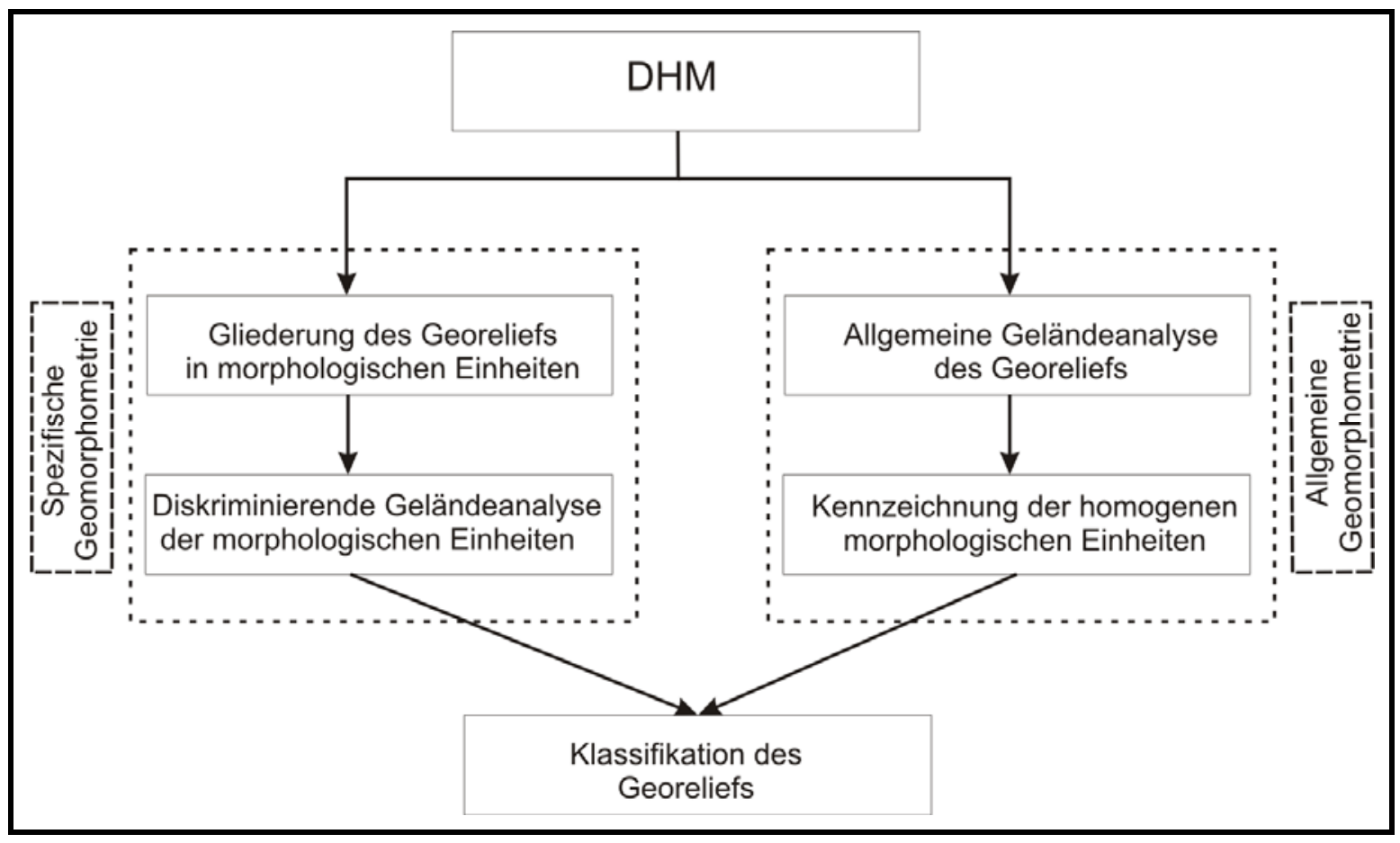

Abb. 5-1: Die geomorphometrische Darstellung auf Basis des DHM 


\subsubsection{Ermittlung der Einzugsgebiete und Subeinzugsgebiete}

Das Einzugsgebiet stellt eine geomorphogenetische Einheit der geomorphologischen Prozesse, insbesondere des fluvialen Erosionsreliefs, dar. Es ist eine der topographischen Einheiten, die für die geomorphologische und geomorphometrische Charakterisierung des Georeliefs sehr gut geeignet ist. Die Betrachtung des Einzugsgebiets bildet in zahlreichen Studien die Basiseinheit vieler geomorphologischer und geomorphometrischer Analysen (CHORLEY, 1969; HoRMANN, 1971). Auf Grund des starken Georeliefeinflusses auf Fließgewässer und auf den festländischen Wasserkreislauf wurden hydrologische Einzugsgebiete aus der geomorphologischen Sicht mehrfach untersucht. Hinzu kommt die große Rolle der geomorphometrischen Indizes des Einzugsgebiets wie die hypsometrische Kurve und das hypsometrische Integral, die sehr nützlich für das Kennzeichnen bestimmter Eigenschaften eines Gebiets sein können, z. B. Niveau der tektonischen Tätigkeiten (KELLER \& PINTER, 2002).

Die Ableitung von Einzugsgebieten erfolgte vor dem Einsatz digitaler Höhenmodelle auf manuellem Weg. Zumeist wurden die benachbarten Einzugsgebiete auf Basis visueller Analysen topographischer Karten abgegrenzt. Der Verlauf von Isohypsen und Gerinnen ermöglicht die Kennzeichnung der Wasserscheiden und Mündungspunkte von Einzugsgebieten. Mit der zunehmenden Verfügbarkeit digitaler Höhendaten begann die Entwicklung von Algorithmen zur computergestützten Ableitung hydrologisch relevanter Informationen (MOORE et al., 1991), wie Fließrichtung, Fließakkumulation und Einzugsgebiete. Diese Algorithmen sind zurzeit standardmäßig in geographischen Informationssystemen implementiert. Die Weiterentwicklung der Algorithmen vor allem zur Verbesserung hydrologischer und hydrogeologischer Modellierungen ist Gegenstand gegenwärtiger Forschung. Die Geomorphometrie von Einzugsgebieten etablierte sich damit als beachtliche Forschungsrichtung innerhalb der Geomorphologie. Dieser bedient sich der klaren und einfachen Definition der Einzugsgebiete bzw. der Wasserscheiden.

In dem betrachteten Gebiet lässt die Hydrographie in Verlauf und Abgrenzung sowohl von der strukturellen Gliederung als auch von der orographisch-morphologischen Beschaffenheit enge Abhängigkeiten erkennen (WOLFART, 1967). Im Bezug darauf, ist die Betrachtung des hydrologischen Einzugsgebiets in dieser Arbeit als geomorphologische Untersuchungseinheit von Bedeutung, vor allem für die regionale Darstellung und Visualisierung des Georeliefs. Deswegen wurde zu Beginn der Darstellung die Hauptwasser- 
scheide ermittelt und danach das Untersuchungsgebiet in Teileinzugsgebiete unterteilt. Die zwei Hauptwasserscheiden, die das Gebiet in drei hydrographische Becken (Orontes, Aleppo, Euphrat) gliedert, wurden ermittelt. Es ist ersichtlich, dass diese Darstellung viel genauer als die alte Definition für diese Becken sein kann. Allerdings ist diese Betrachtung sowohl für die hydrologischen als auch für die geomorphologischen Untersuchungen sehr allgemein und unvollständig. Diese Beurteilung lässt sich ausdrücklich für das hydrographische Becken von Aleppo bestätigen, weil hier drei Bereiche zu unterscheiden sind, die verschiedene geomorphologische und hydrologische Eigenschaften haben, insbesondere im Bezug auf die vorherrschenden geomorphologischen Prozesse. Diese Teileinzugsgebiete sind: das Teileinzugsgebiet des Al-Jabboul, das Teileinzugsgebiet von Qoueiq und das Teileinzugsgebiet Al-Match und von Al-Kharaitch (Abb. 5-2).

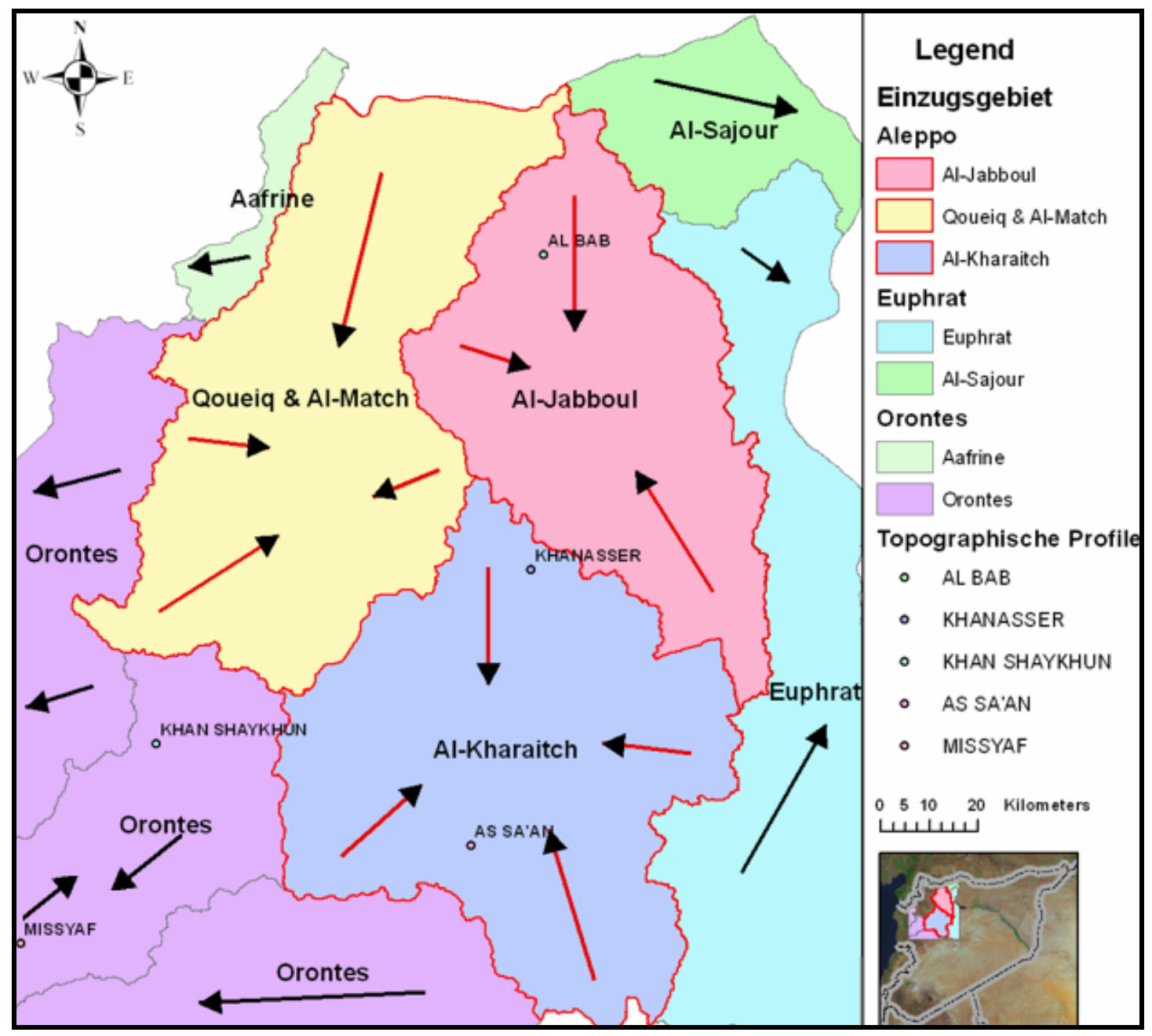

Abb. 5-2: Gliederung des Untersuchunggebiets in Teileinzugsgebieten 
Im Westen lassen sich auch zwei Teileinzugsgebiete unterscheiden: das Subeinzugsgebiet von Aafrine im Nordwesten und das Subeinzugsgebiet vom mittleren Orontes im Südwesten. Im Osten ist das Gebiet praktisch ausschließlich als Euphrat Einzugsgebiet zu kennzeichnen. Dennoch lässt sich ein Subeinzugsgebiet in der nordöstlichen Ecke benennen. Es entwässert in Richtung des Al-Sajour, der seinerseits in den Euphrat mündet.

Topographische Profile in W-E-Richtung (ihre Positionen in Abb. 5-2) können das Georelief und die Lage der Wasserscheiden deutlicher sichtbar machen. Eindeutig lässt sich der große Unterschied zwischen dem Vorflutniveaus der inneren Einzugsgebiete (bei Qoueiq ca. 240m ü. NN, im Norden von Al-Jabboul ca. 300m ü. NN) und der westlichen Einzugsgebiete (Orontes sowie Aafrin ca. 100-180m ü. NN) erkennen. Im Gegenzug sind kaum Unterschiede zwischen dem Niveau des im Osten angrenzenden Euphrat-Tals und demjenigen des Einzugsgebiets von Al-Jabboul praktisch festzustellen.

Im Süden kann das Georelief durch zwei Profile (Missyaf und Al Sàan) dargestellt werden (Abb. 5-3). Das Profil von Missyaf stellt das Georelief östlich und westlich des Orontes dar. Der westliche Teil des Profils durchquert das Plateau von Missyaf. Der östliche Teil des Profils zeigt, wie das Georelief über eine lange Strecke kleine Hangneigungen (das Vorland von Palmyra-Ketten) aufweist und erst in den PalmyraKetten eine gewisse Höhe (mehr als 900m ü. NN) erreicht. Das Profil Al Sàan überquert das DSFS und das Orontes-Tal im Westen und das Vorland der Palmyra-Ketten im Osten.

Der Zentralbereich des Gebiets wurde mittels zweier Profile (Khan Shaykhun, Khanasir) dargestellt (Abb. 5-4). Sie kreuzen den Zawiye und repräsentieren seinen Steilhang im Westen sowie seine sukzessive Abdachung nach Osten. Weiter nach Osten lässt sich die Landschaft der abflusslosen Becken (Al-Matah und Al-Jabboul) und Basaltdecken von Hama, dem Shbith und Elhass zeigen. Nördlich von der Stadt Aleppo kann die Landoberfläche mittels des Profils von Al-Bab dargestellt werden. Dieses Profil weist im Westen wie die anderen Profile einen steilen Hang zum Aafrin-Tal auf, der unter Wirkung der Verwerfung von Aafrin (ein Teil vom DSFS) entstand. Nach Osten durchquert das Profil das Qoueiq Tal und das hügelige Land des Al-Bab Plateaus (Abb. 5-4). 


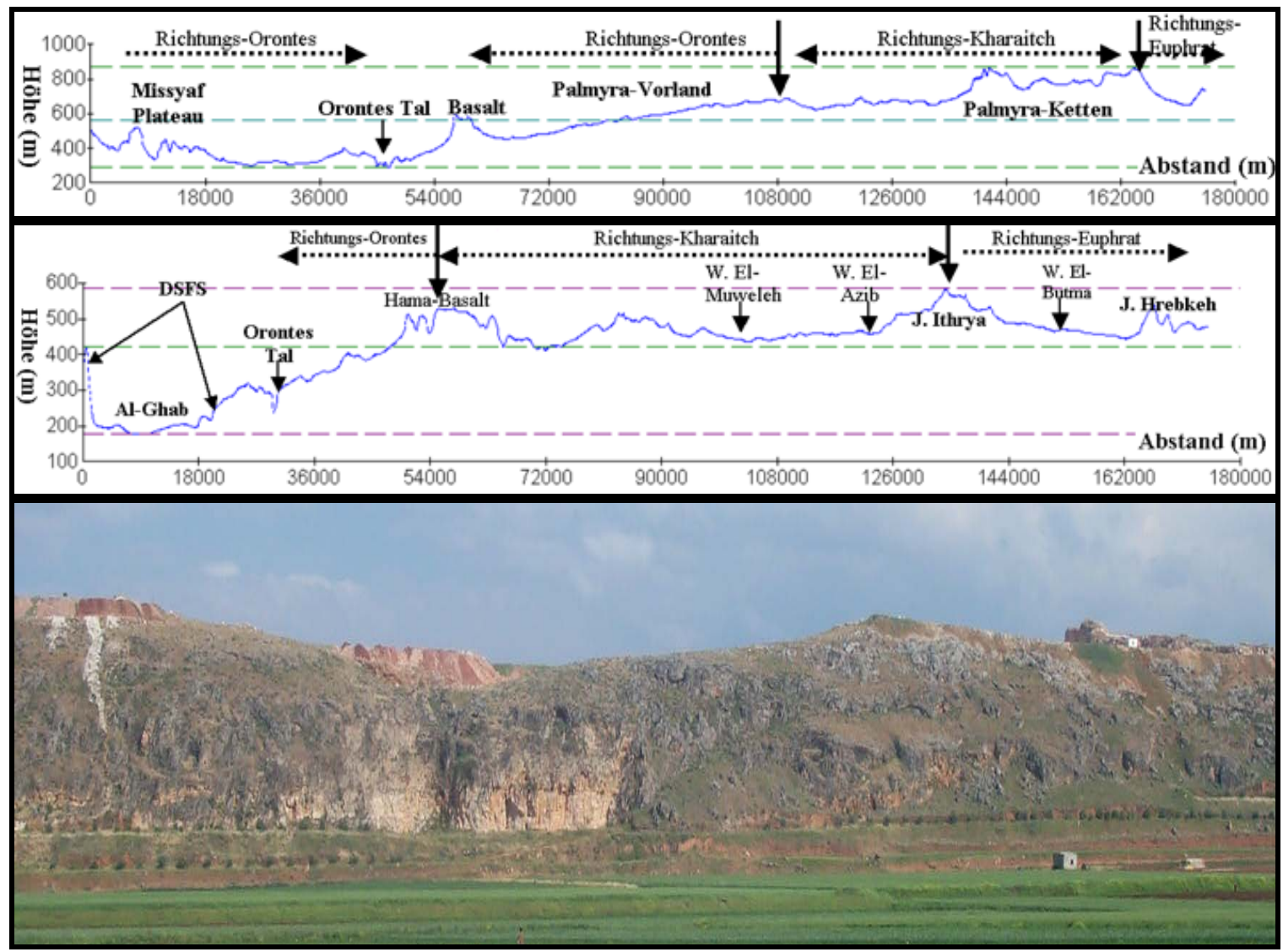

Abb. 5-3: Profil Missyaf (oben), Al Sàan (Mitte) und Foto des DSFS-Hangs (Ausblick nach Osten)

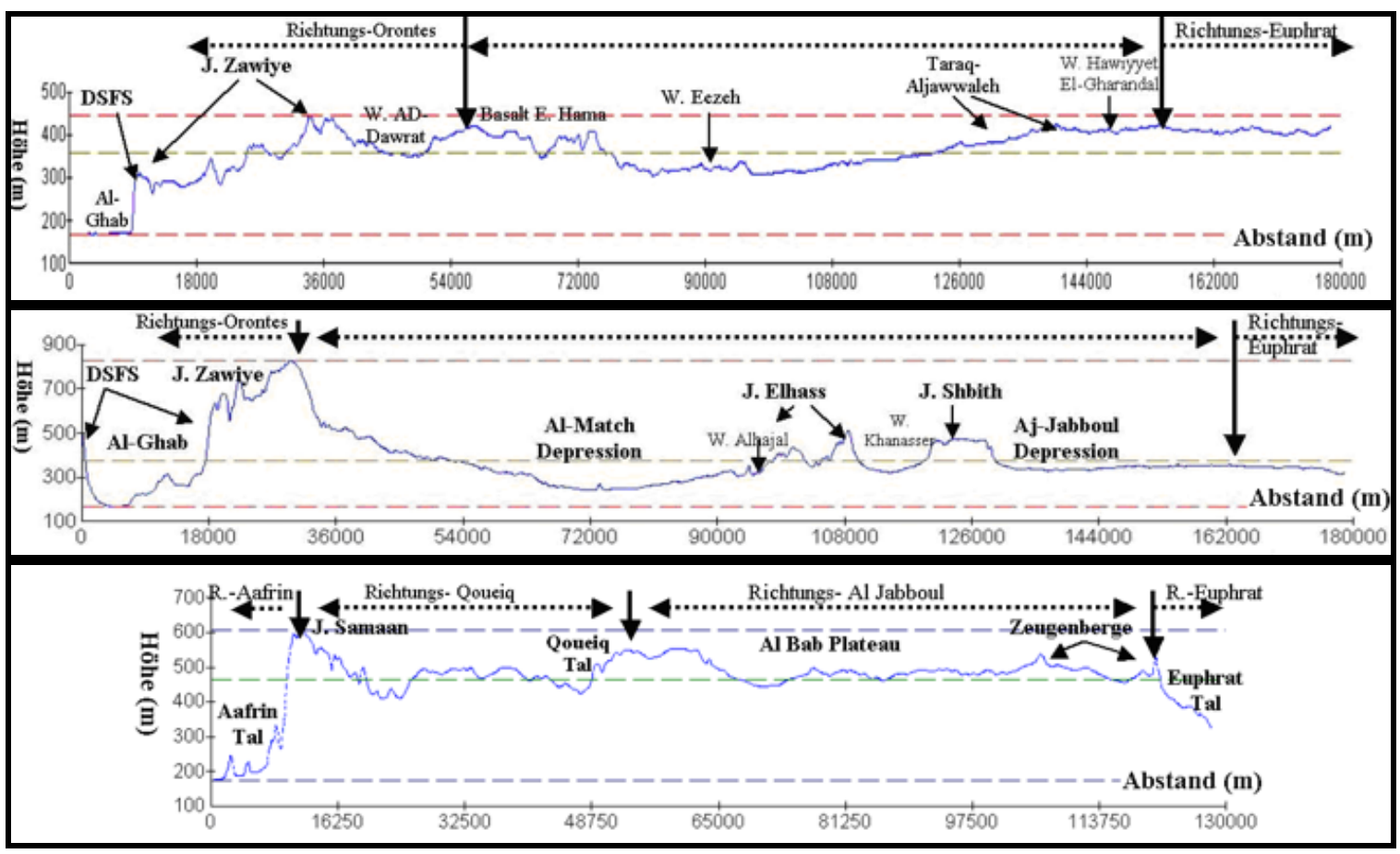

Abb. 5-4: Das Profil Khan Shaykhun (oben), Khanasser (Mitte) und Al-Bab (unten) 


\subsubsection{Die Ermittlung der geomorphometrischen Parameter}

Die aus dem digitalen Höhenmodell ableitenden Reliefparameter, insbesondere Höhe (mittlere absolute Höhe, relatives Relief) und Hangneigung, stellen numerische Wertebereiche dar, die bezüglich einer Interpretation für den Geomorphologen direkt einsetzbar sind. Sie können im Hinblick auf die digitale Georeliefanalyse für eine Vielzahl von Aufgabenstellungen als geomorphometrische Größen verwendet werden. Hierzu zählt zunächst die Klassifizierung, Kennzeichnung und Visualisierung der räumlichen und elementaren Struktur und Georeliefgestalt. Im Folgenden werden diese Aspekte kurz erläutert und eingesetzt, um die Hauptreliefeinheiten zu beschreiben. Die Ableitung der geomorphometrischen Parameter erfolgte im ArcGIS (ArcMap), speziell mit dem Erweiterungstool 3D-Analyst.

\subsubsection{Die Höhe (relatives Relief, absolute Höhe)}

Die Höhe in Meter ü. NN (die absolute Höhe) oder als relative Höhe im Bezug zum niedrigsten Punkt des jeweiligen Untersuchungsgebiets kann als Indikator für die potentielle Energie betrachtet werden. Dieser geomorphometrische Parameter wurde bereits am Ende des 19. Jahrhunderts verwendet.

In der Literatur findet man mehrere Definitionen für das relative Relief. Die meisten Autoren bezeichneten es als die Höhendistanz vom Gipfel zu einem benachbarten Talboden. Manche Autoren wie BARSCH \& CAINE (1984) drückten es als Höhendistanz in Meter pro Quadratkilometer aus. Dieser Begriff wurde bereits von PENK (1894) analog zur Abgrenzung von Hügel und Berg eingeführt. Im deutschsprachigen Raum wurde der Begriff des relativen Reliefs lange Zeit als Reliefenergie bezeichnet. PASSARGE (1921) unterschied auf Basis des relativen Reliefs die Typen Flachland (ca. $50 \mathrm{~m}$ ), Hügelland (50 - $200 \mathrm{~m}$ ), Bergland (200 - $500 \mathrm{~m}$ ), Mittelgebirge (500-1000 m) und Hochgebirge (> $1000 \mathrm{~m}$ ). Die absolute Höhe bezieht sich auf den Meeresspiegel. Es wurde auch wie das relative Relief in vielen alten Publikationen zur Charakterisierung und Abgrenzung von den Georeliefeinheiten (Ritter, 1817; Penck, 1894; Passarge, 1921; Hagedorn \& Poser, 1974) verwendet. PASSARGE (1921) unterschied auf Basis der absoluten Höhe in die Kategorien Tiefland, Mittelland, Hochland, und Alpenland (Tab. 5-2). Eine Kombination des relativen Reliefs und der absoluten Höhe zur Abgrenzung von Relieftypen nahm PASSARGE nicht vor. 
In der Tat dient der Parameter der absoluten Höhe nur als zusätzlich kennzeichnendes Attribut und muss mit anderen Parametern kombiniert werden, um das Georelief genau zu segmentieren. Der Parameter der absoluten Höhe kann dennoch in dem Untersuchungsgebiet verwendet werden, um eine primäre Klassifizierung bzw. Visualisierung für das Georelief zu vermitteln. Im Bezug auf das alte Muster von PASSARge (1921) für die Reliefgliederung auf Basis dieses Parameters ist das ganze Untersuchungsgebiet gewissermaßen als Mittelland zu bezeichnen. Die tektonischen Becken vom Al-Ghab und ein Teil des Aafrine Flusstals im Westen lassen sich nicht unter dem Begriff Mittelland zusammenfassen, sondern sind laut des Musters unter der Kategorie Tiefland aufzugliedern. Zur Vereinfachung der Georeliefdarstellung wurde das Gebiet auf Basis der absoluten Höhe in neun Klassen unterteilt (Abb. 5-5).

Tab. 5-2: Die geomorphologische Klassifikation auf Basis der absoluten Höhe

\begin{tabular}{|c|c|c|l|}
\hline \multicolumn{2}{|c|}{ PASSARGE (1921) } & \multicolumn{1}{c|}{ HAGEDORN (1974) } \\
\hline $\begin{array}{c}\text { Höhe } \\
(\mathrm{m} \text { ü. NN) }\end{array}$ & Georelief & $\begin{array}{c}\text { Höhe } \\
(\mathrm{m} \text { ü. NN) }\end{array}$ & \multicolumn{1}{|c|}{ Georelief } \\
\hline $0-200$ & Tiefland & $0-30$ & Schwaches (Flachland, Ebene) \\
\hline $200-1500$ & Mittelland & $30-200$ & Mäßiges (Hügelland) \\
\hline $1500-3000$ & Hochland & $200-1000$ & Mittleres (Bergland, Mittelgebirge) \\
\hline$>3000$ & Alpenland & $>1000$ & Starkes (Gebirgsland, Hochgebirge) \\
\hline
\end{tabular}

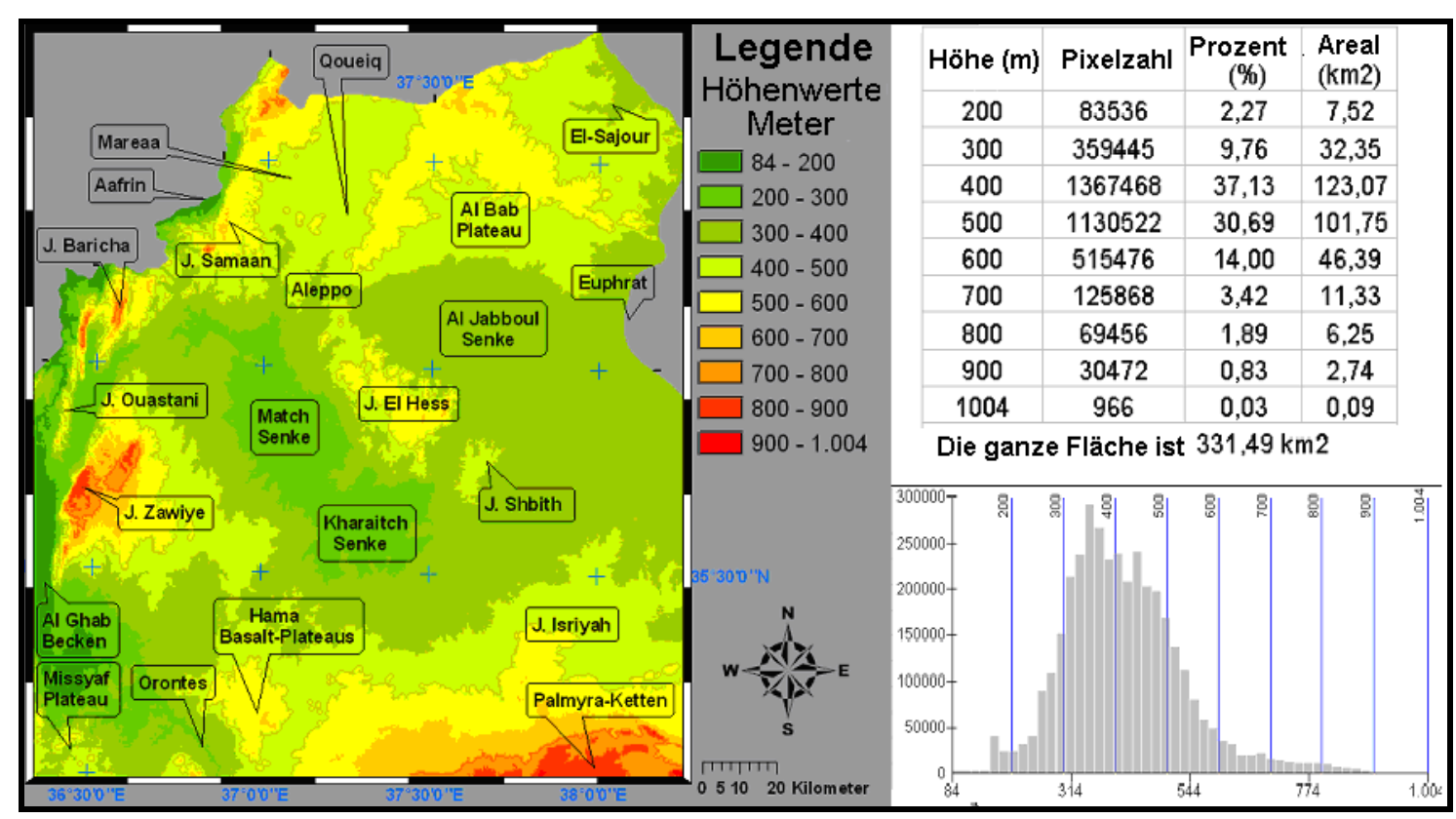

Abb. 5-5: Auf Basis der absoluten Höhe klassifiziertes DHM (links) und Statistische Auswertungen (rechts) 


\subsubsection{Die Hangneigung}

Hangneigung (Länge des Gradienten) ist die Neigung in Richtung der größten Höhenänderung des betrachteten Punktes zu den Nachbarpunkten oder Angabe des Winkels von der Horizontalen zum Bezugspunkt in Grad, Promille oder Steigung. Der Parameter der Hangneigung ist die wichtigste Größe für die Kennzeichnung von der Georeliefgestalt. In der Veröffentlichung von DEMEK et al. (1982) ist eine gute Klassifikation der Hangneigungswinkel in Gruppen und Bereichen (Tab. 5-3) zu finden. Dabei wurden mehrere Relieftypen genannt, deren Hangneigung in einem bestimmten Bereich liegt, z. B. für den Hangneigungsbereich $\left(1^{\circ}-3^{\circ}\right)$ wurden die Terrassen-, Kammflächen und die Hochflächen der Zwischentalbereiche erwähnt.

Tab. 5-3: Hangneigungstypen (DEMEK et al., 1982)

\begin{tabular}{|c|c|c|c|}
\hline \multicolumn{3}{|c|}{ Winkelgrade } & \multirow{3}{*}{$\begin{array}{l}\text { Bezeichnung } \\
\text { Ebene (eben) }\end{array}$} \\
\hline Gruppen & \multicolumn{2}{|c|}{ Bereiche } & \\
\hline $0^{\circ}$ & $0^{\circ}$ & $0^{\circ}$ & \\
\hline \multirow{3}{*}{$1^{\circ}-7^{\circ}$} & \multirow{2}{*}{$1^{\circ}-3^{\circ}$} & $1^{\circ}$ & \multirow{3}{*}{ Flachhang (flach) } \\
\hline & & $2^{\circ}-3^{\circ}$ & \\
\hline & $4^{\circ}-7^{\circ}$ & $4^{\circ}-7^{\circ}$ & \\
\hline \multirow{3}{*}{$8^{\circ}-25^{\circ}$} & \multirow{2}{*}{$8^{\circ}-15^{\circ}$} & $8^{\circ}-11^{\circ}$ & \multirow{3}{*}{$\begin{array}{l}\text { mäßig geneigter Hang } \\
\text { (mäßig geneigt) }\end{array}$} \\
\hline & & $12^{\circ}-15^{\circ}$ & \\
\hline & $16^{\circ}-25^{\circ}$ & $16^{\circ}-25^{\circ}$ & \\
\hline \multirow{3}{*}{$26^{\circ}-60^{\circ}$} & $26^{\circ}-35^{\circ}$ & $26^{\circ}-35^{\circ}$ & \multirow{3}{*}{ Steilhang (steil) } \\
\hline & \multirow{2}{*}{$36^{\circ}-60^{\circ}$} & $36^{\circ}-45^{\circ}$ & \\
\hline & & $46^{\circ}-60^{\circ}$ & \\
\hline $61^{\circ}-90^{\circ}$ & $61^{\circ}-90^{\circ}$ & $61^{\circ}-90^{\circ}$ & Wand (wandig) \\
\hline $90^{\circ}$ & $90^{\circ}$ & $90^{\circ}$ & Überhang (überhängig) \\
\hline
\end{tabular}

Die Hangneigungsanalyse neben dem oberen Höhenmuster und deren Verteilung ermöglicht eine sehr gute Diskretisierung des räumlichen Georeliefs in Einheiten, die mit den anderen Konzepten dieser geomorphologischen Darstellung in einer engen Beziehung stehen. Um die differenziert morphologische Ausprägung möglichst repräsentativ abzubilden, wurde eine eigene Hangneigungsunterteilung entwickelt. In dem betrachteten Gebiet ergab sich eine maximale Hangneigung von 46,615 $5^{\circ}$ Daraus wurden insgesamt sieben Hangneigungsklassen $\left(0^{\circ}-2^{\circ}, 2-4^{\circ}, 4-8^{\circ}, 8-16^{\circ}, 16-32^{\circ}, 32-40^{\circ}, 40-\right.$ 46,615 ) unterschieden, die die Ausprägung der Reliefelemente, insbesondere die Schichtstufen, gut widerspiegeln. Diese lässt die Schichtstufen nordöstlich von Aleppo und im Norden sowie Nordwesten von den Palmyra-Ketten deutlich erkennen. 
Die Häufigkeitsverteilung der Hangneigung dient in der Geomorphologie einer ersten Beschreibung des Georeliefcharakters eines Gebietes. Auf Basis der Form der Histogramme lassen sich erste Aussagen über die Werteverteilung der Variablen treffen.

Die statistische Analyse der Hangneigung (Abb. 5-6) zeigt eindeutig an, dass ein größerer Teil des betrachteten Gebiets eine Hangneigung unter $40 \mathrm{Grad}$ hat. Landschaftsformen mit einem Hangneigungsgradienten über 40 Grad (Steilhänge) treten in einer begrenzten Anzahl auf. Sie befinden sich in Bereichen, in denen die tektonischen Aktivitäten das Gebiet stark beeinflussen. Diese sind insbesondere im Westen festzustellen, wo meist junge, oft noch anhaltende tektonische Bewegungen verbunden mit einer komplexen geologischen Struktur zusammenwirken und zu dieser oben beschriebenen besonders starken Hangneigung führen. Die starke Hangneigung ist auch in den Bereichen, wo der Basalt die Landoberfläche verhüllt (Basaltdecken), zu beobachten. Darüber hinaus sind vereinzelte Steilhänge auch bei aufgesetzten Schichttafeln und einigen Zeugenbergen anzutreffen.

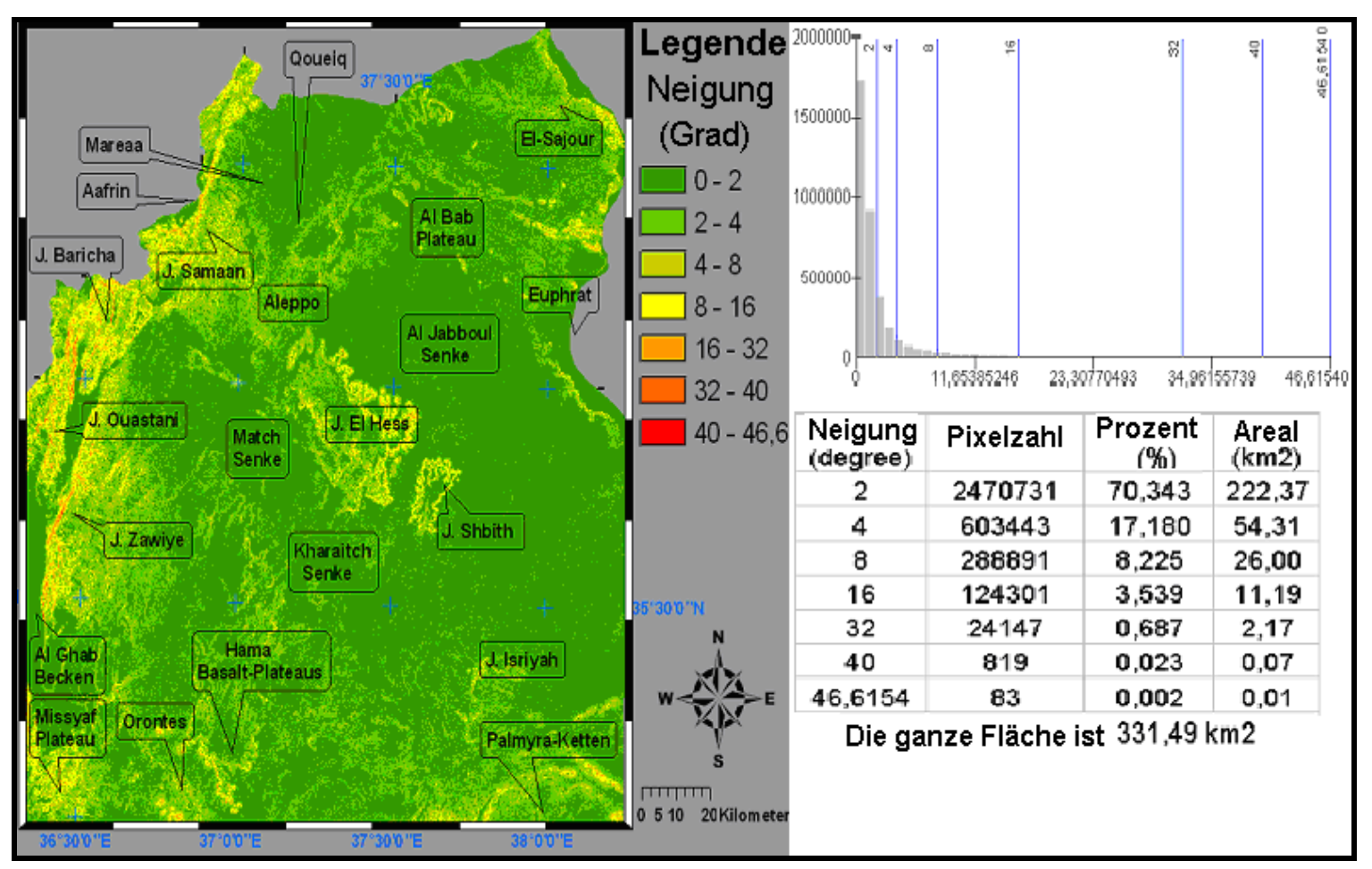

Abb. 5-6: Karte der Hangneigung und Häufigkeitsverteilung 


\subsubsection{Die Darstellung der morphologischen Haupteinheiten}

Die Ausgliederung und Abgrenzung sowie die kartographische Darstellung der Georeliefeinheiten ist in der Literatur als geomorphologische Regionalisierung bezeichnet. Diese beruht auf die Typisierung und Klassifizierung der Georeliefformen nach einer der o. g. geomorphologischen Darstellungen (DEMEK, 1976).

Das Untersuchungsgebiet wurde von WOLFART (1967) als Tafelland bezeichnet, in dem wie oben erwähnt flache Schichtlagerungen vorherrschen. Das Gebiet unterlag während der verschiedenen geologischen Zeiten mehreren Einebnungsprozessen (Kapitel 2.1.2). Das Zentrum des Untersuchungsgebiets ist von Rumpflandschaften geprägt, die durch fehlende tektonische Aktivitäten und lang anhaltende Erosion mit Ablagerungen in abflusslosen Becken entstanden sind.

Die Darstellung von dem Georelief (Haupteinheiten) wurde mittels der computergestützten Visualisierungsverfahren des digitalen Höhenmodells durchgeführt. Solche Verfahren sind zurzeit sehr gebräuchlich geworden, weil geographische Softwarepakete mit unterschiedlichen Techniken das dreidimensionale Georelief (3D) perspektivisch visualisieren können. Diese Techniken schließen die Anwendung von den Höhenlinien (Abb. 5-7) und dem geschummerten Reliefmodell etc. ein (KRAAK \& ORMELING, 2003).

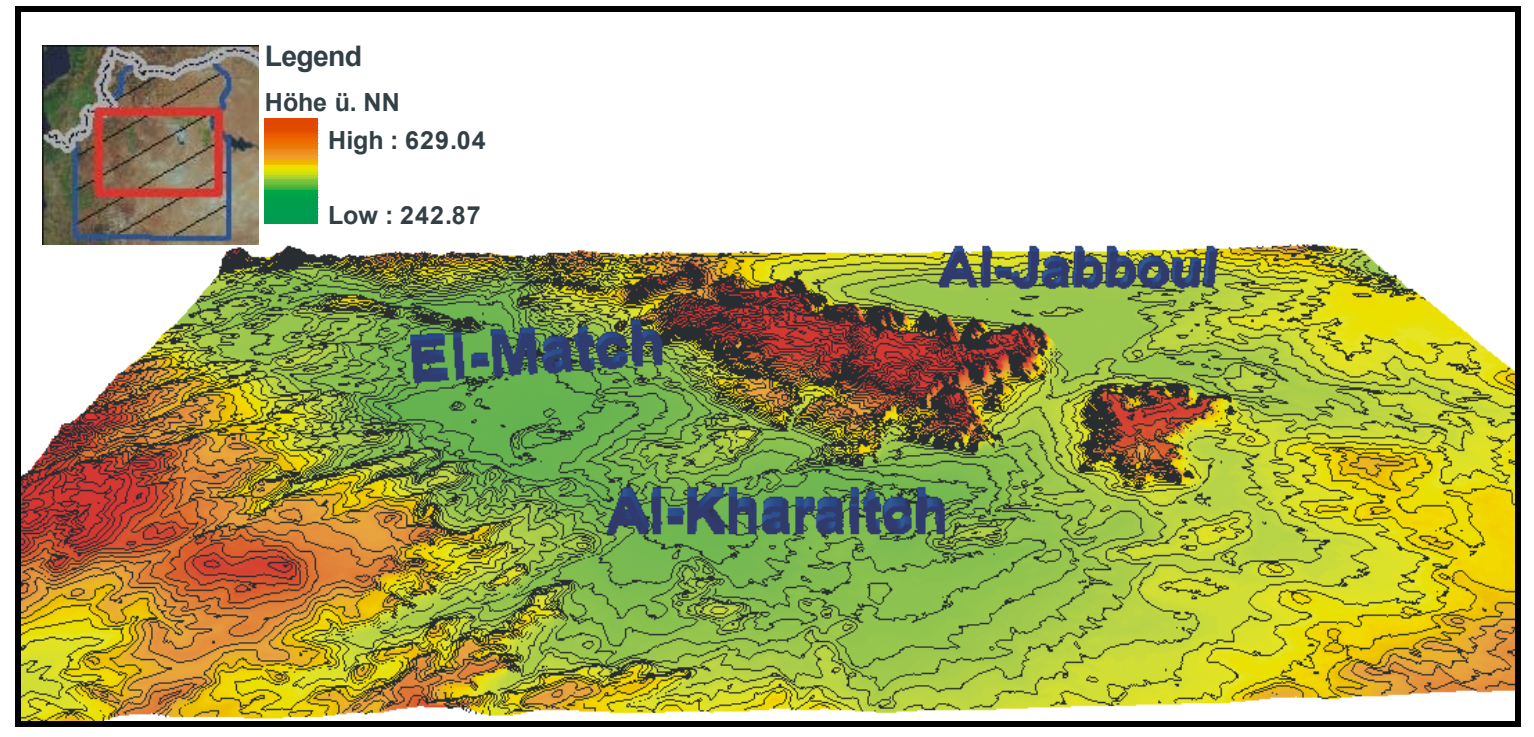

Abb. 5-7: Darstellung der Geomorphologie mittels der Höhenlinien (Basis ist DHM) 
Das geschummerte Reliefmodell ist sehr „populär“ und am gebräuchlichsten, weil es subtile Variationen des Georeliefs hervorhebt und ihre realistische Beschreibung und Deutung erlaubt (BRASSEL, 1974; HoRN, 1981; ONORATI et al., 1992). Dabei wird die Beleuchtung des Reliefs aus einer wählbaren Richtung und Höhe über dem Horizont simuliert. Das geschummerte Reliefmodell und die Höhendaten können mit thematischen Informationen, beispielsweise der Wölbung oder der Hangneigung, verschnitten werden und ermöglichen dadurch eine erste Interpretation von berechneten geomorphometrischen Parametern (THELIN \& PIKE, 1991). Daher findet das geschummerte Reliefmodell eine verstärkte und verbreitete Anwendung in der geomorphologischen Analyse v. a. in der Morphotektonik und der Extraktion der Strukturen im regionalen Maßstab (SMITH \& CLARK, 2005). Diese Bedeutungen werden bei der Ermittlung von linearen Elementen in einem anderen Teil (Kapitel 6.2.1) dieser Arbeit diskutiert. Das Modul ArcScene in dem geographischen Informationssystem ArcGIS bietet die Möglichkeit, alle o. g. perspektivischen Darstellungen von Landoberflächen auf Basis des digitalen Höhenmodells durchzuführen. Es erlaubt zusätzlich die Verknüpfung von den Satelliten- oder Luftbildern mit den digitalen Höhendaten (Abb. 5-8).

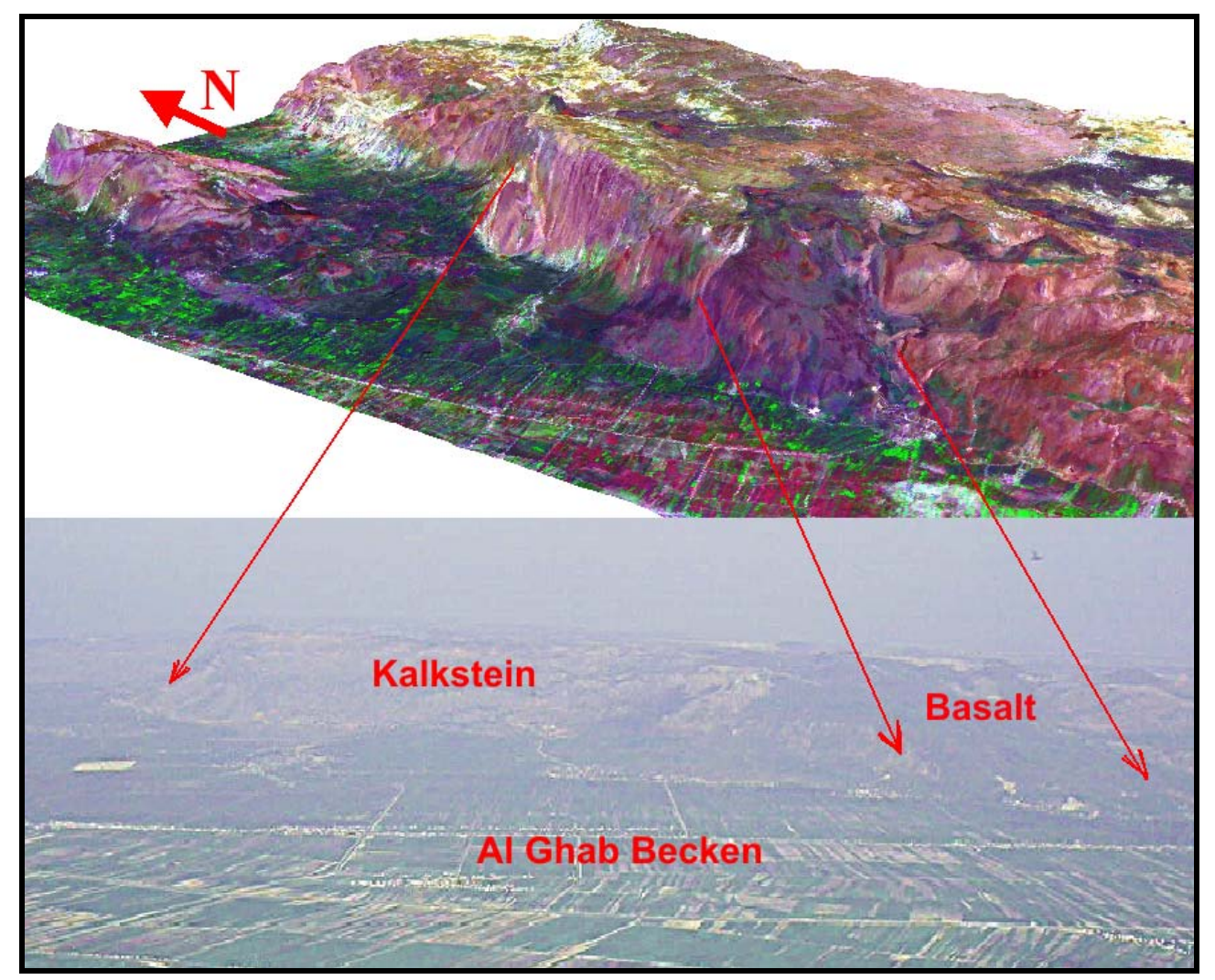

Abb. 5-8: Überlagerung von Landsat ETM+ (741) und DHM (DSFS) Ausblick nach Osten 
Per Tastatur oder Maus ist eine Navigation im Darstellungsraum möglich. Somit ist das digitale Höhenmodell in der Verbindung mit Satellitenbildern sehr nützlich für Vorstellungen und Hervorhebungen der großen Strukturen, welche ansonsten photogeologische Interpretationen und Feldbeobachtungen erfordern. Diese Methode wird für Analysen und Darstellungszwecken der strukturellen Geländeeigenschaften stets verwendet. Sie bietet bei vielen Schritten dieser Arbeit einen ausdrucksvollen Ersatz der vorörtlichen Angabe.

Durch Betrachtung der obigen Darstellungen und die Anwendung des Rasterrechners können die morphologischen Haupteinheiten des Georeliefs gekennzeichnet werden. Die morphologischen Einheiten mit einer absoluten Höhe $\geq 200 \mathrm{~m}$ und einer Hangneigung $\geq 15 \%$ wurden berechnet. Diese Berechnung ergibt Rasterdaten mit zwei Werten (0 und 1), die durch Konvertierung als Shapefile gespeichert wurden. Polygone mit dem Wert „Null“ wurden gelöscht, weil sie den oben genannten Parametern nicht entsprechen. Die festgehaltenen Polygone mit dem Wert „Eins“ segmentieren die morphologischen Haupteinheiten. Diese thematische Karte kann auf Satellitenbildern oder anderen Rasterdaten wie das klassifizierte digitale Höhenmodell überlagert werden, um die weitere Beschreibung zu unterstützen (Abb. 5-9).

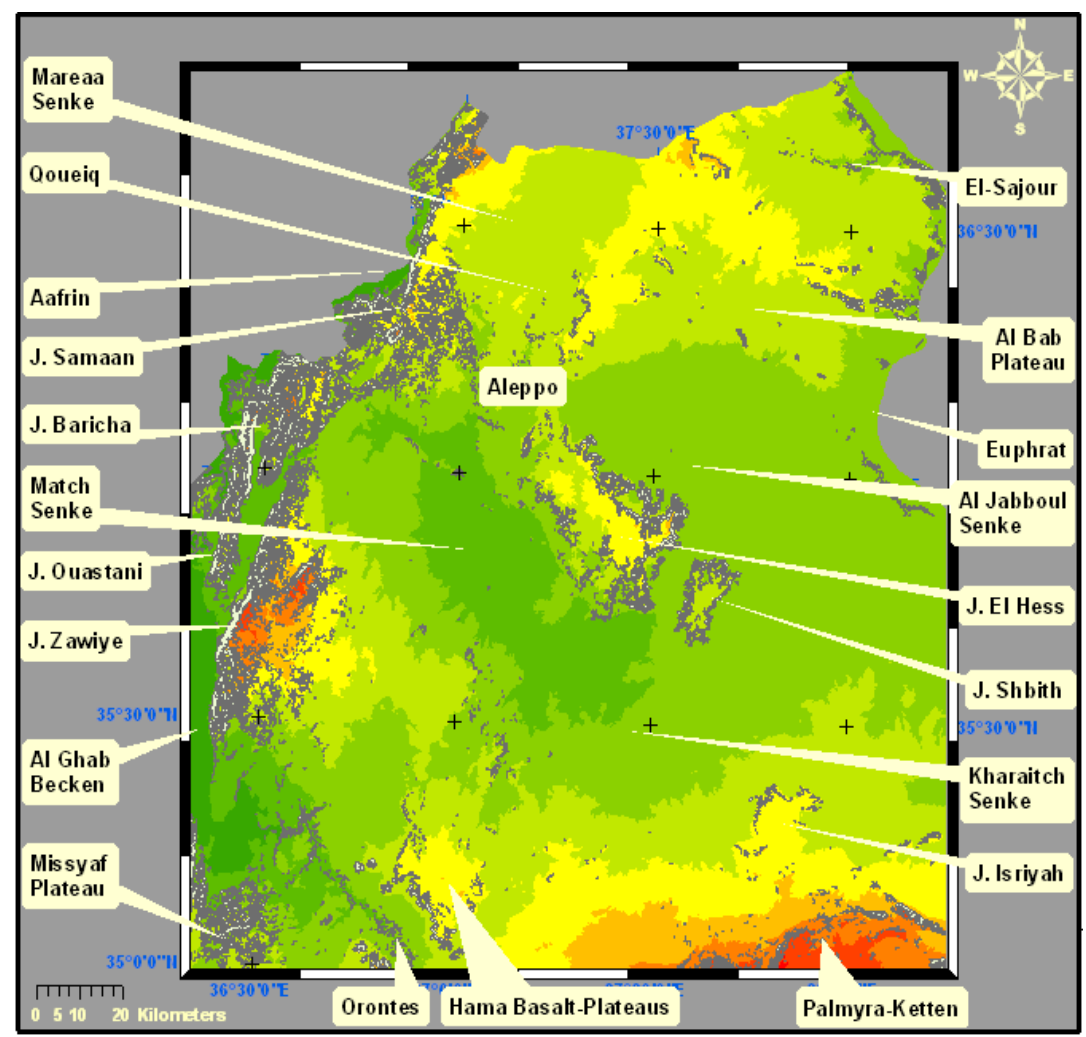

Abb. 5-9: Segmentierung der morphologischen Haupteinheiten (graue Polygone) überlagert auf DHM 
Unter Berücksichtigung dieser Visualisierung und der Geologie des Untersuchungsgebiets lassen sich die in der Tabelle (5-4) aufgelisteten morphologischen Reliefeinheiten unterscheiden.

Tab. 5-4: Georelief-Einheiten des Untersuchungsgebiets

\begin{tabular}{|c|c|l|l|}
\hline $\begin{array}{c}\text { Kategorie } \\
\text { der } \\
\text { absoluten } \\
\text { Höhen }\end{array}$ & $\begin{array}{c}\text { Absolute } \\
\text { Höhe } \\
\text { (m ü. } \text { NN) }\end{array}$ & \multicolumn{1}{|c|}{ Georelief-Einheiten } & \multicolumn{1}{|c|}{ Geologie } \\
\hline Tiefland & $0-200$ & $\bullet$ Tektonische Becken & $\begin{array}{l}\text { Auffüllung von } \\
\text { quartärer Ablagerung }\end{array}$ \\
\hline \multirow{2}{*}{ Mittelland } & $200-400$ & $\begin{array}{l}\bullet \text { Täler der Hauptflüsse } \\
\bullet \text { Senke }\end{array}$ & $\begin{array}{l}\text { Auffüllung von } \\
\text { quartärer Ablagerung }\end{array}$ \\
\cline { 2 - 4 } & $400-700$ & $\begin{array}{l}\bullet \text { Hügelige horstartige Plateaus } \\
\bullet \text { Zeugenberge (Plateaureste) }\end{array}$ & $\begin{array}{l}\text { Kalksteinbänke } \\
\text { Basaltdecken }\end{array}$ \\
\cline { 2 - 4 } & $700-1000$ & $\bullet$ Horstzone und Bergland & Kalksteinbänke \\
\hline
\end{tabular}

\subsubsection{Täler der Hauptflüsse}

Hierzu gehören die Täler von Orontes, Aafrin, Qoueiq, El-Sajour und Euphrat. Ein Teil des Orontes (der obere) befindet sich außerhalb des betrachteten Gebiets. Der mittlere Orontes fließt im Südwesten des Untersuchungsgebiets mäandrierend durch sein Tal. Sein Niveau liegt hier etwa zwischen 275m (Profil 1) und 225m (Abb. 5-10), während es direkt westlich dieses Bereichs im Becken von Al-Ghab bei ca. 170m liegt. Der Orontes hat hier steil abfallende Uferwände von beträchtlicher Höhe geschaffen (Abb. 5-10). Im Süden lässt sich eine scharfe Umbiegung des Orontes nach Nordwesten beobachten. Die schluchtähnliche Gestalt und die scharfe Umbiegung des Tals deuten zweifellos auf eine tektonische Wirkung hin.

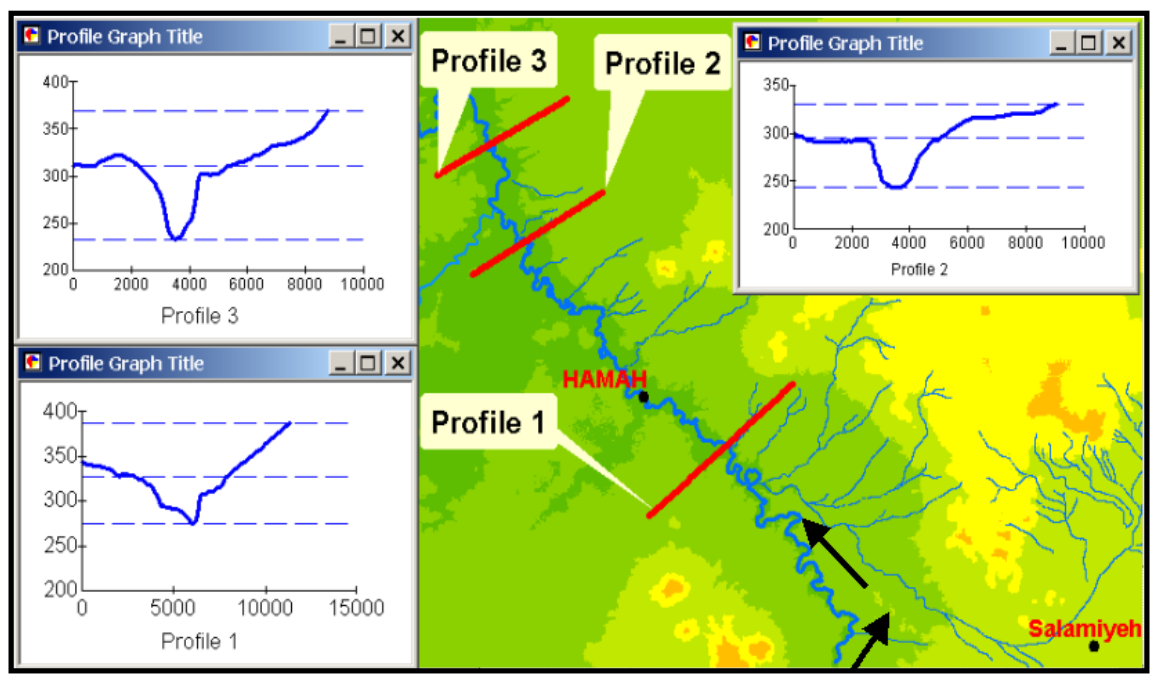

Abb. 5-10: Topographische Profile durch den Orontes (Pfeile markieren die Umbiegung) 
Die Morphologie des Tals von Aafrin sowie des Tals von Euphrat ist bereits oben auf dem Profil von Al-Bab zu erkennen. Auf Grund, dass es sich bei diesen beiden Flüssen um Grenzen des betrachteten Gebiets handelt, werden sie hier nicht weiter behandelt. Während diese beiden Flüsse sowie der Orontes über das ganze Jahr Wasserzufluss haben, fällt der Qoueiq auf syrischem Territorium zeitweise trocken, weil er zurzeit bereits auf der türkischen Seite gestaut wird. Der Fluss Qoueiq entspringt an der Flanke des türkischen Taurusgebirges und durchquert das betrachtete Gebiet annähernd meridional. Ein Teil des Qoueiq-Tals, das ein Niveau unter 400m Meereshöhe hat, fängt 10km nördlich der Stadt Aleppo an und zeigt auch wie der Orontes den Charakter eines Schlucht-Tals (Abb. 5-11). Hier treten die Plateauränder zusammen und verengen das Tal bis auf $300 \mathrm{~m}$ Breite. Nördlich davon fließt er mäandrierend, ohne ein ausgeprägtes Tal zu bilden, auf einer weiten Ebene. Die Umbiegung und der eindeutige Schluchtcharakter südlich von Aleppo sind wie bei dem Orontes auf tektonische Einflüsse zurückzuführen.

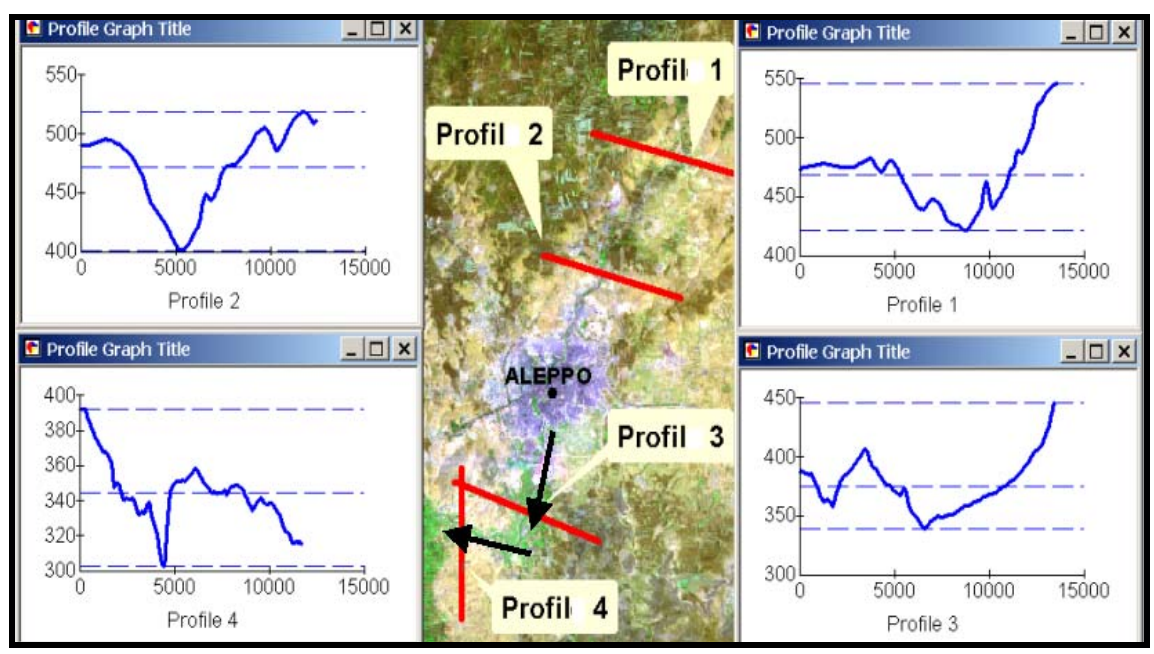

Abb. 5-11: Topographische Profile durch den Qoueiq

\subsubsection{Die topographischen Senken}

Die topographischen Senken treten in den abflusslosen Becken: i. Salzsee von AlJabboul, ii. das Endsee von El-Match und iii. das Sabkha von Al-Kharaitch auf (Abb. 57). Die Morphologie dieser Becken sowie die Morphologie ihrer Grenzen lassen sich nicht unabhängig von der tektonischen Tätigkeit interpretieren. Nach WOLFART (1967) sanken (Kapitel 2.1.2) diese Becken am Ende Pliozän/Anfang Pleistozän in einer Zeit großer tektonischer Aktivität ein und bildeten die strukturelle Grundlage für die Entstehung quartärer Seen sowie den überwiegend endorheischen Abflüssen (Kapitel 2.1.2). In Richtung dieser Becken entwässern alle Wadis des inneren Arbeitsgebiets. 


\subsubsection{Die Kalkstein-Plateaus}

Gebiete mit Plateaucharakter lassen sich in drei Bereiche erkennen: Missyaf, Zawiye \& Samaan sowie Al-Bab (Abb. 5-9). Das Plateau von Missyaf liegt südwestlich des Untersuchungsgebiets zwischen dem Orontes bei Hama und des DSFSs bzw. der Küstengebirge. Das Niveau des Plateaus ist ca. 450m Meereshöhe. Im Vergleich zu seiner Umgebung ist hier ein unruhiges Relief zu beobachten (Abb. 5-12).

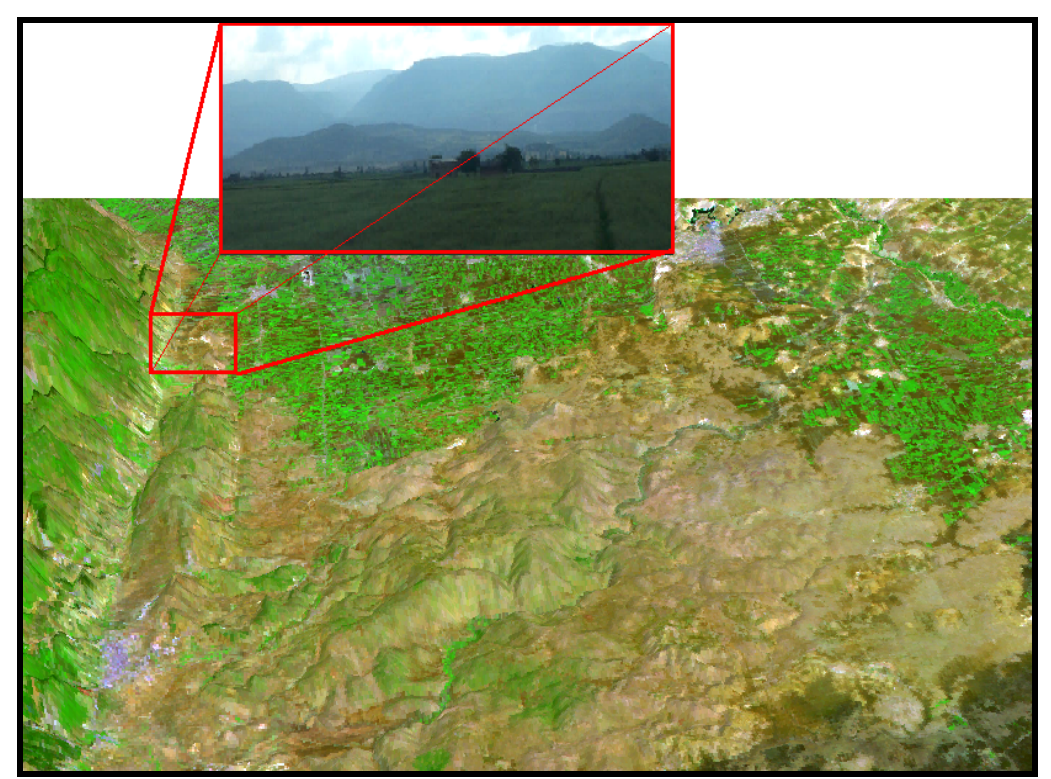

Abb. 5-12: Missyaf-Plateau (Überlagerung der Kombination 741 über das DHM)

Das Plateau von J. Zawiye und J. Samaan dehnt sich nördlich vom Missyaf sowie östlich der Ebene vom Al-Ghab aus. Die höchste absolute Höhe erreicht im J. Zawiye 939m und im J. Samaan 870m. Das Georelief weist hier ausschließlich nach Westnordwesten orientierte steile Hänge und ein bedeutsames relatives Relief auf. Nach Osten senken sich die Plateaus sanft ab, wobei sie zu einem Schollenland zu zählen sind (Abb. 13 \& 14).

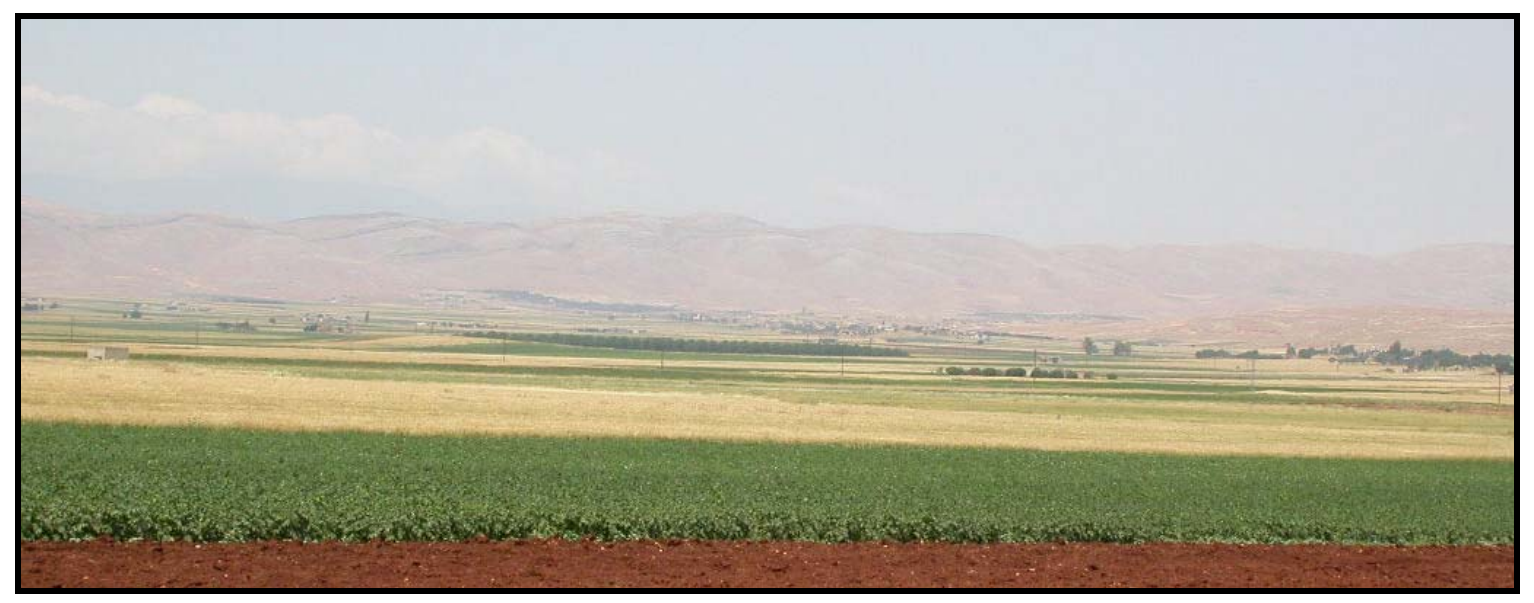

Abb. 5-13: Abdachung des Zawiye nach Osten (Ausblick nach Westen) 


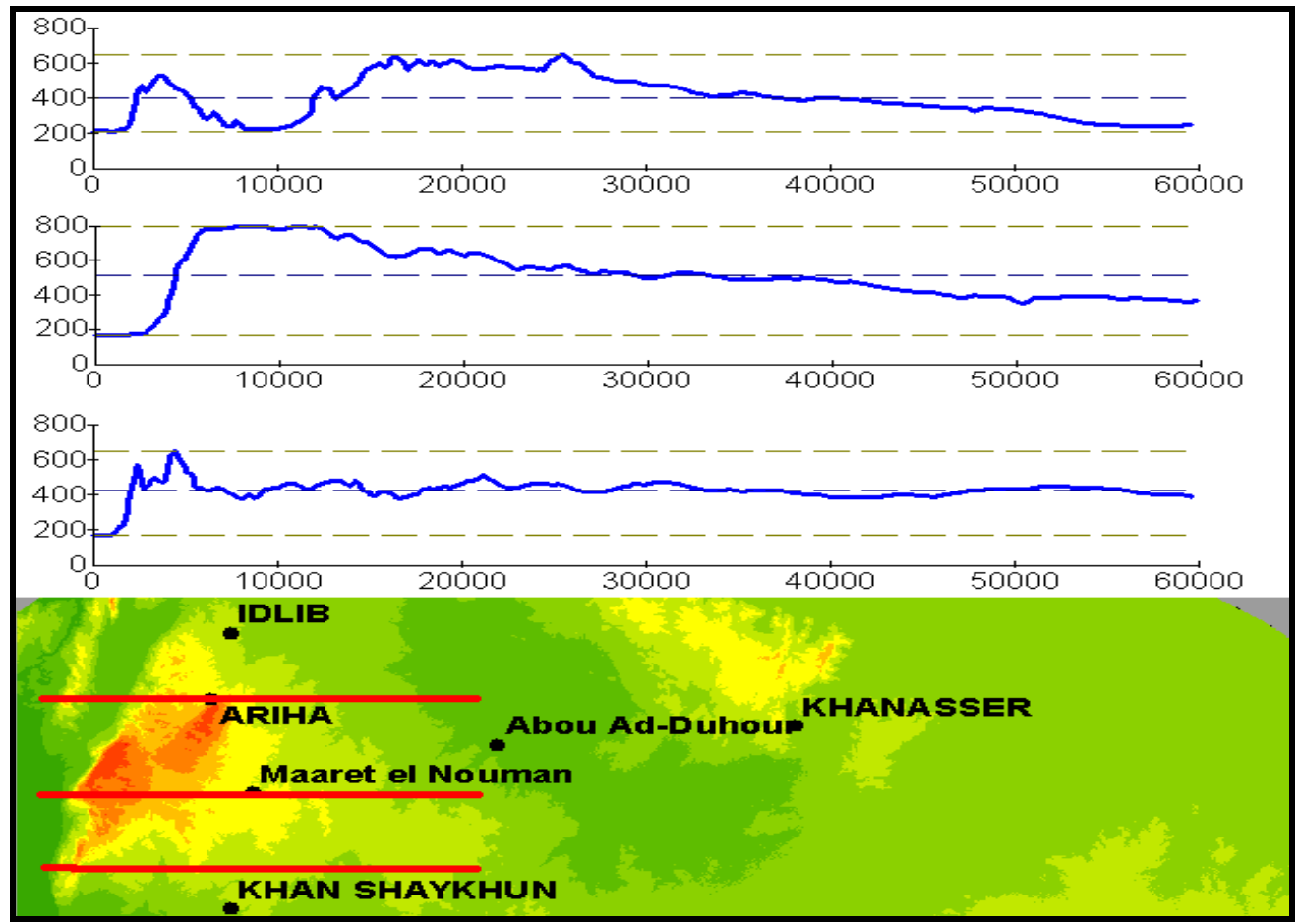

Abb. 5-14: Topographische Profile durch den Zawiye

Das Al-Bab Plateau liegt östlich vom J. Samaan zwischen der Stadt Aleppo und dem Euphrat in einem Niveau von $400 \mathrm{~m}$ bis zu $600 \mathrm{~m}$. Durch die Betrachtung der dreidimensionalen Darstellung kann das Plateau als Pediplain bezeichnet werden, da es sich in mehrere Einzelflächen gliedern lässt (Abb. 5-15). Im Osten geht das Plateau mit leicht unebenen Reliefexpressionen schrittweise in die Euphratebene über.

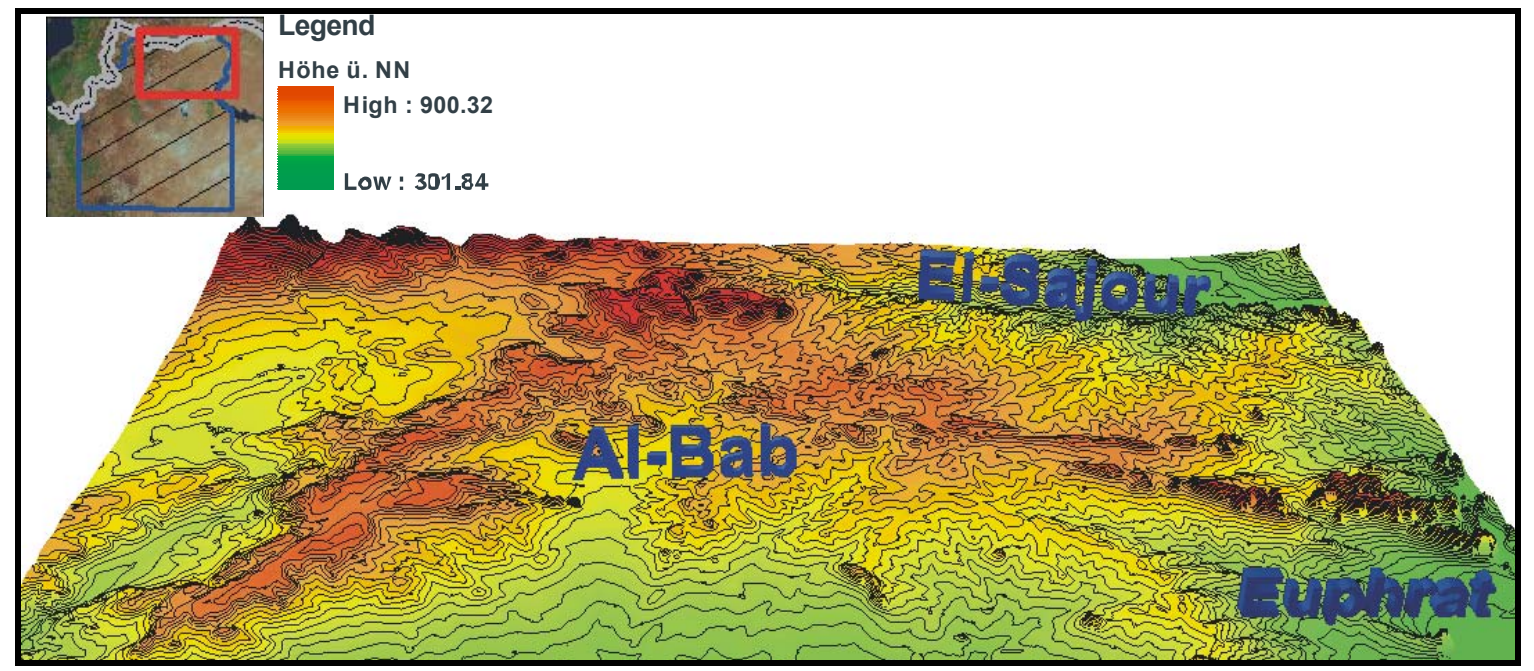

Abb. 5-15: 3D-Darstellung des Al-Bab Plateaus 


\subsubsection{Die Basalt-Plateaus}

Die Georeliefformen sind stellenweise über große Areale des Untersuchungsgebiets durch basaltischen Vulkanismus geprägt. Drei riesige Basaltfelder lassen sich in dem Gebiet unterscheiden. Das Shbith $\left(160 \mathrm{~km}^{2}\right)$ und das El Hess $\left(8800 \mathrm{~km}^{2}\right)$ trennen die o. g. abflusslosen Becken voneinander (Abb. 5-9). Das dritte Basaltfeld dehnt sich östlich von Hama nach Norden bis zum Zentrum des Arbeitsgebiets aus und grenzt die Senke von Al-Kharaitch im Westen ab. Auf den topographischen Profilen stellen sich die Lavafelder als flache, schwach zertalte Tafeln dar. Im Süden östlich von Hama ragen sie in einer bis zu 300m hohen Stufe über ihr Umland auf (Abb. 5-16). Im Norden östlich von Maarret el Nouman geht das Basaltfeld von Hama ohne sichtbare Geländestufen in das umliegende tertiäre Tafelland über.

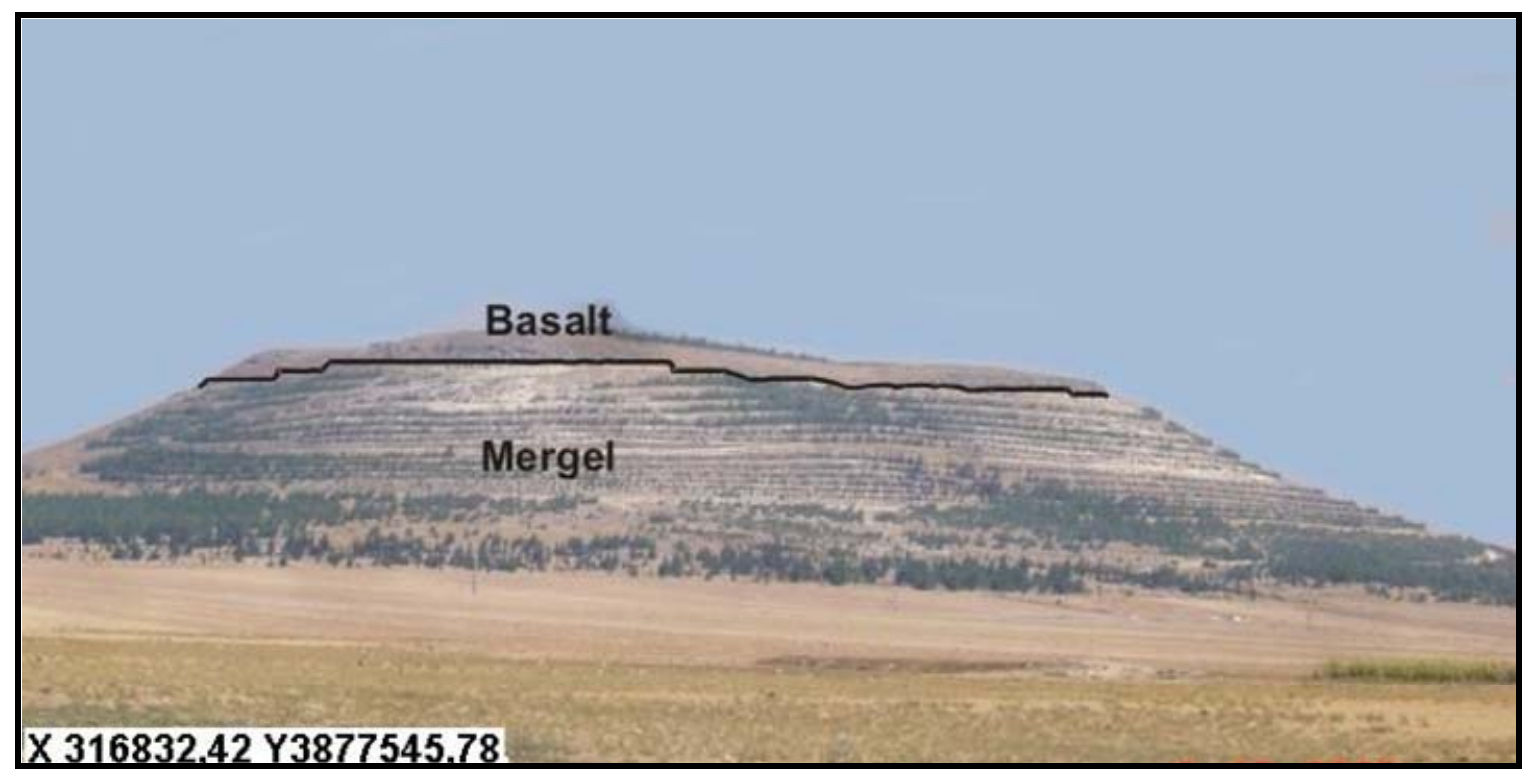

Abb. 5-16: Übergang von der Basaltstufe und dem liegenden weißen Mergel

\subsubsection{Das Bergland}

Georeliefformen mit bergigen Charakteren sind im Südosten des Untersuchungsgebiets zu beobachten. Das bergige Georelief im Südosten gehört den nördlichen Palmyra-Ketten an. Sie ragen hier bis zu 1000m über NN. Das Georelief ist durch weitgespannte Hochflächen und breite Höhenrücken geprägt, die eine leichte Abdachung nach Norden besitzen. In den Profilen lassen sich deutlich schluchtähnliche Täler erkennen (Abb. 5-3), die das Bergland zerschneiden. Starke Zertalung und Schichtstufen prägen weiterhin das Georeliefbild am Fuß dieser Höhen. 


\subsection{Ermittlung der Geomorphologie mittels Satellitenbilder}

Die Ermittlung der geomorphologischen Karte ist das wichtigste Arbeits- und Darstellungsmittel bei geomorphologischen Untersuchungen, weil sie einerseits die Möglichkeit $\mathrm{zu}$ einer flächendeckenden quasi quantitativen Aussage geben und andererseits die konkrete Verbindung zu Nachbardisziplinen herstellen kann. Diesbezüglich sollte eine ausführliche geomorphologische Darstellung bzw. Kartierung die Landformen mit Informationen über die Lithologie und Hydrographie kombinieren (KLIMASZEWSKI, 1990).

Seitdem die Fernerkundungstechnologie zum Einsatz gebracht wurde, wurden Kartierungsverfahren in großem Maße geändert. Zurzeit haben Fernerkundungstechniken eine große Bedeutung gewonnen und sind ein wesentlicher Bestandteil eines Kartierungsprogramms geworden, besonders im Hinblick auf regionale Niveaus. Die geokodierten Satellitendaten in Form von fotographischen Drucken (Bilder) dienen den Feldgeologen und Feldgeomorphologen als sehr gut geeignete Datenquelle. Nach LIST et al. (1990) kann eine Satellitenaufnahme im weitesten Sinne als topographisches und geologisches Kartieren verstanden werden. Nach ABER et al. (1993) sind Landsatbilder ideale Quellen der Informationen für die geomorphologischen Karten in den mittleren Maßstäben von 1:100.000 oder kleiner. In diesem Rahmen lassen die Satellitenbilder sich nicht nur zur Erstellung einer Basiskarte benutzen, sondern viel wichtiger als Anwendung für die systematische Erfassung geomorphologischer Daten. Auf Grund dessen wurde diese im folgenden Kapital berücksichtigt.

Die lithologischen Hauptbestandteile der morphologischen Einheiten sind Kalkstein, Kreide, Mergel, Basalt und quartäre Ablagerungen. Für die Beschreibung bzw. die Kartierung der Geomorphologie der o. g. morphologischen Einheiten gelten sowohl die visuellen als auch digitalen Auswertungsverfahren der Satellitenbilder als vollständige erfolgreiche Methode. 


\subsubsection{Auswahl der Kanalkombinationen und der Bilder}

Für eine ausführliche Ergänzung der oben durchgeführten morphologischen Darstellung war die Anwendung von mehreren Verfahren notwendig, um eine vollständige Beschreibung der verschiedenen geomorphologischen Phänomene zu erreichen.

Zuerst wurden geeignete Kanalkombinationen definiert, da für die Auswertung grundsätzlich beliebig viele Falsch-Farbkompositionen FCCs (False colour composites) von Landsatbildern bearbeitet werden können (Kapitel 5-1-2). Die Auswahl der optimalen Bandkombinationen für die Beschreibung bzw. Kartierung der Geomorphologie wurde einleitend durch das rechnergestützte Verfahren auf dem statistischen Weg verwirklicht. Diesem dient das berühmte rechnergestützte Verfahren von CHAVEZ et al. (1982), das als Optimum-Indexfaktor (OIF) bezeichnet wird. Das Verfahren beruht auf eine Berechnung eines statistischen Werts. Es berechnet mit Hilfe der Standardabweichung und der Korrelationskoeffizienten die informativste Kanalkombination des spektralen Datensatzes. Dementsprechend wurden die drei signifikantesten Kanäle mit Hilfe des Faktors (OIF) von den Eingabebildern ausgehend errechnet (Tab. 5-5).

Tab. 5-5: OIF-Verteilung für RGB-Kombinationen der verwendeten Landsatdaten

\begin{tabular}{|c|c|c|c|c|}
\hline Kanale- Kombinationen & \multicolumn{4}{|c|}{ Optimum-Indexfaktor-Werten } \\
\hline & $\mathbf{1 7 4 0 3 5}$ & $\mathbf{1 7 4 0 3 6}$ & $\mathbf{1 7 3 0 3 5}$ & $\mathbf{1 7 3 0 3 6}$ \\
\hline $\mathbf{1 2 3}$ & 9,137 & 8,347 & 5,261 & 7,071 \\
\hline $\mathbf{1 2 4}$ & 9,119 & 8,224 & 5,418 & 6,760 \\
\hline $\mathbf{1 2 5}$ & 8,627 & 8,381 & 5,600 & 7,023 \\
\hline $\mathbf{1 2 7}$ & 8,615 & 8,091 & 5,543 & 6,793 \\
\hline $\mathbf{2 3 4}$ & 9,399 & 8,879 & 6,051 & 7,336 \\
\hline $\mathbf{2 3 5}$ & 8,813 & 8,945 & 6,056 & 7,593 \\
\hline $\mathbf{2 3 7}$ & 8,812 & 8,722 & 6,184 & 7,358 \\
\hline $\mathbf{3 4 5}$ & 8,806 & 9,421 & 6,483 & 7,715 \\
\hline $\mathbf{3 4 7}$ & 8,786 & 9,169 & 6,597 & 7,483 \\
\hline $\mathbf{4 5 7}$ & 8,252 & 9,125 & 6,298 & 7,412 \\
\hline $\mathbf{1 3 4}$ & 9,201 & 8,834 & 6,043 & 7,207 \\
\hline $\mathbf{1 3 5}$ & 8,622 & 8,932 & 6,092 & 7,468 \\
\hline $\mathbf{1 3 7}$ & 8,578 & 8,630 & 6,032 & 7,234 \\
\hline $\mathbf{1 4 5}$ & 8,612 & 8,802 & 6,189 & 7,148 \\
\hline $\mathbf{1 4 7}$ & 8,634 & 8,573 & 6,305 & 6,922 \\
\hline $\mathbf{1 5 7}$ & 8,041 & 8,637 & 6,100 & 7,169 \\
\hline $\mathbf{2 4 5}$ & 8,888 & 8,865 & 6,112 & 7,282 \\
\hline $\mathbf{2 4 7}$ & 8,901 & 8,623 & 6,224 & 7,054 \\
\hline $\mathbf{2 5 7}$ & 8,297 & 8,655 & 5,995 & 7,297 \\
\hline $\mathbf{3 5 7}$ & 8,111 & 9,132 & 6,223 & 7,722 \\
\hline
\end{tabular}


Diese Werte wurden als Leitfaden für die Überprüfung von mehreren Kombinationen verwendet (Abb. 5-17). Dabei wurde berücksichtigt, dass die Kanäle des sichtbaren Spektrums (1, 2 und 3), und die Kanäle des Mittleren Infrarots (5 und 7) untereinander relativ stark miteinander korreliert sind (MULDERS, 1987). Infolgedessen ergab sich die Beobachtung, dass die informativste Darstellung in der Regel einen Kanal aus dem MIR (TM-5 oder TM-7) beinhaltet. Der OIF wurde u. a. in den Arbeiten von DwIVEDI (1992) und HILDEBRANDT (1992) erfolgreich verwendet.

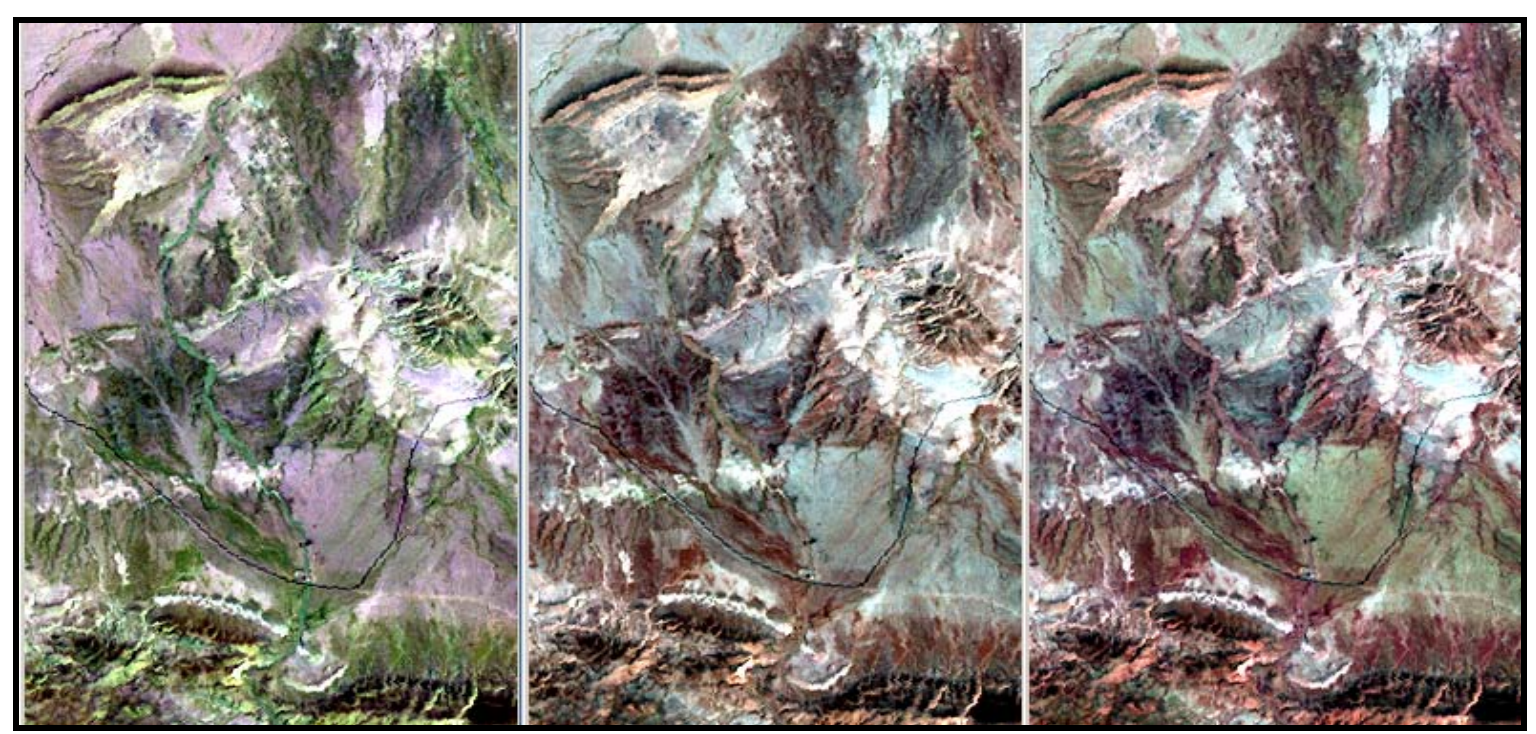

Abb. 5-17: Die RGB-Kombinationen mit dem höchsten OIF der Szene ETM 173036 (Links 753, Mitte 543, Rechts 532)

Für die Interpretation der Satellitenbilder wurden auch Hauptkomponentenbilder aus den sechs multispektralen Kanälen des Landsat ETM+ Systems herangezogen. Die Kombination RGB der ersten drei Kanäle dieser produzierten Daten war sehr nützlich für die Diskriminierung der lithologischen Einheiten insbesondere im Bereich der Palmyra-Ketten (Abb. 4-12), wodurch die verschiedenen lithologischen Einheiten der Kreidezeit sowie ihre Verwitterungsprodukte kontrastreich dargestellt werden können. Die Zurücktransformation dieser HKA-Daten in das ursprüngliche Koordinatensystem (Kapitel 4.3.2.4), was auch als inverse HKA bekannt ist (WILLIAMS, 1983), ergibt Daten mit einer verbesserten Farbauflösung, da das Rauschen der höheren Hauptkomponente nicht mehr auftaucht. 


\subsubsection{Mosaikbildung}

Für die geomorphologische Beschreibung auf Basis der Satellitendaten war als erstes eine Mosaikbildung erforderlich, um ein für das ganze Untersuchungsgebiet flächendeckendes Raster hervorzurufen. Im Kapitel 3.2 wurde erwähnt, dass das Untersuchungsgebiet von vier Subszenen abgedeckt ist. Eine unmittelbare Mosaikbildung von den vorhandenen Subszenen auf Pixelebene ist unmöglich, da die Szenen nicht direkt hintereinander bei demselben Überflug aufgenommen wurden. Darüber hinaus besitzen die vier Satellitenszenen unterschiedliche Bildeigenschaften bezüglich atmosphärischer Verhältnisse, Vegetation, Geometrie usw.

Bei der Mosaikbildung war vor allem die Korrektur der Fehler notwendig, die unter Einfluss des Sonnenstands und Erde-Sonne-Abstands entstehen, wodurch die topographischen und atmosphärischen Effekte eliminiert werden können (LILLESAND \& KIEFER 2000). Die Angleichung der Bildeigenschaften der unterschiedlichen Aufnahmen konnte erst nach der Anwendung mehrerer Verfahren (geometrische und atmosphärische Korrektur sowie Histogrammanpassung) erreicht werden (Abb. 5-18).

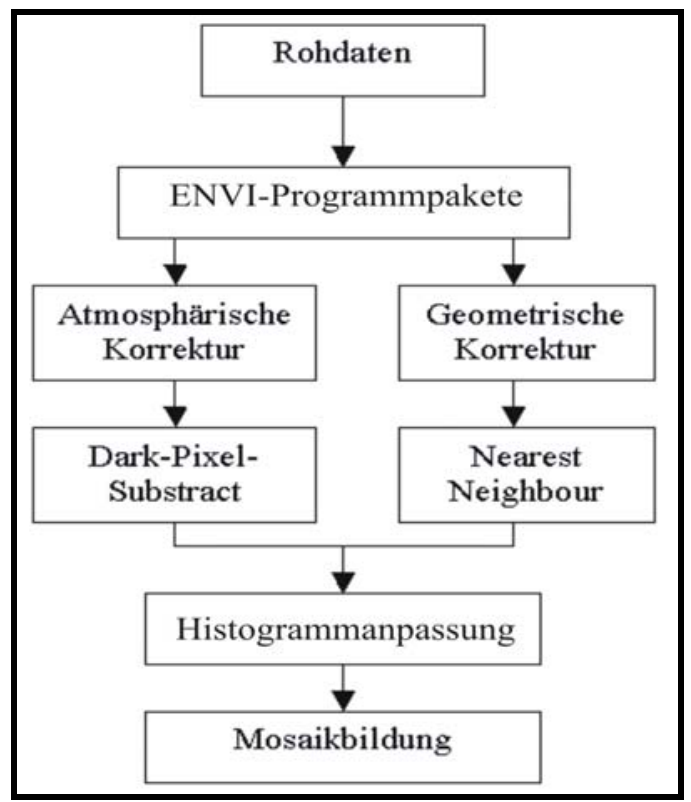

Abb. 5-18: Ablaufdiagramm der Mosaikbildung

Auf diese Weise war die Mosaikbildung von den vier ETM+ Szenen auf Kartenebene möglich. Diese Szenen bestehen aus zwei Szenen des Path 173 von 18.08.2000 (17305 \& 17306) und des Path 174 von 22.06 .2000 (174035 \& 174036). Die Szene von dem Path 174 wurden mit der ,high gain“ Stufe aufgenommen und von dem Path 173 mit „low gain“, welche eine größere Helligkeit der Pathszenen 174 im Vergleich mit 173 bewirkt. 
Die Vegetationsabdeckung im westlichen Bereich des Gebietes (Path 174) ist viel ausgedehnter als im Östlichen, welche eine unterschiedliche Reflexion verursacht. Diese störenden Faktoren konnten durch die Normalisierung und Histogrammanpassung beseitigt werden (Abb. 5-18).

Für die Mosaikbildung wurde die Kanalkombination 741 ausgewählt (Siehe Anhang 3). Dieser Entscheidung liegt zugrunde, dass die Hauptgesteine in dem Untersuchungsgebiet Karbonatgesteine, Basalte und ihre Verwitterungsprodukte sind. Der ETM+ Kanal 7 (SWIR) deckt die Absorptionsbänder von Hydroxylionen und Karbonaten ab und dient als ein Indikator für Tonminerale. Außerdem ermöglicht er eine Separation zwischen sandigen und tonigen Flächen. In dem Bereich des Kanals 4 wird die Strahlung durch die Vegetation stark reflektiert und durch Wasser absorbiert. Diese erhöht den Kontrast zwischen den unverhüllten Gesteinen, der Vegetation und den Böden (insbesondere den feuchten Böden). Zusätzlich ruft der ETM+ Kanal 1 einen höheren Kontrast zu ETM+ Kanal 4 hervor, weil die Strahlung in seinem Bereich von der Vegetation und den FeOxiden absorbiert wird (GRIPPEN, 1989). Darüber hinaus hat diese Kanalkombination den Vorteil, Objekte dem Auge vertrauten Farbtönen wiederzugeben, wobei Wasser in Blau, Vegetation in Grün und Gesteine in Rot-, Gelb- und Brauntönen dargestellt sind. Mehrere Autoren wie Kaufmann \& Pfeiffer (1986) und Kenea(1997) sind der Ansicht, dass diese Kombination für die geologische Auswertung lithologischer und struktureller Eigenschaften in ariden Gebieten von größerer Bedeutung ist.

\subsubsection{Die geomorphologische Auswertung der Satellitendaten}

Für die Visualisierung und Abgrenzung der geomorphologischen Eigenschaften in dem Gebiet wurden mehrere Kanalkombinationen und Produkte verschiedener Fernerkundungsverfahren überprüft. Dabei wurde festgestellt, dass sich einige Kanalkombinationen und Produkte dafür als empfehlenswert bezeichnen lassen. Von sehr gutem Nutzen waren vor allem die Produkte der Ratioverfahren.

Das weit verbreitete geomorphologische Phänomen im Zentrum des Untersuchungsgebiets, bevorzugt in den abflusslosen Becken, sind die versalzten Flächen. Für die Abgrenzung dieser Erscheinung eignet sich eine RGB-Kombination der Ratiobilder 3/1, 5/7 und 3/5 besonders gut, da Salz und versalzte Flächen türkisgrün sowie Wasser dunkellila bis blau dargestellt werden. Bei der Anwendung von dem thermalen Kanal 6 des Landsat 
TM und den Kanälen 1 und 7 war sogar eine Trennung zwischen verschiedenen Graden der Versalzung möglich, z. B. in der Umgebung von dem Al-Jabboul werden die versalzten Oberflächen mit ansteigendem Salzgehalt gelb bis tiefrot abgebildet (Abb. 5-19).

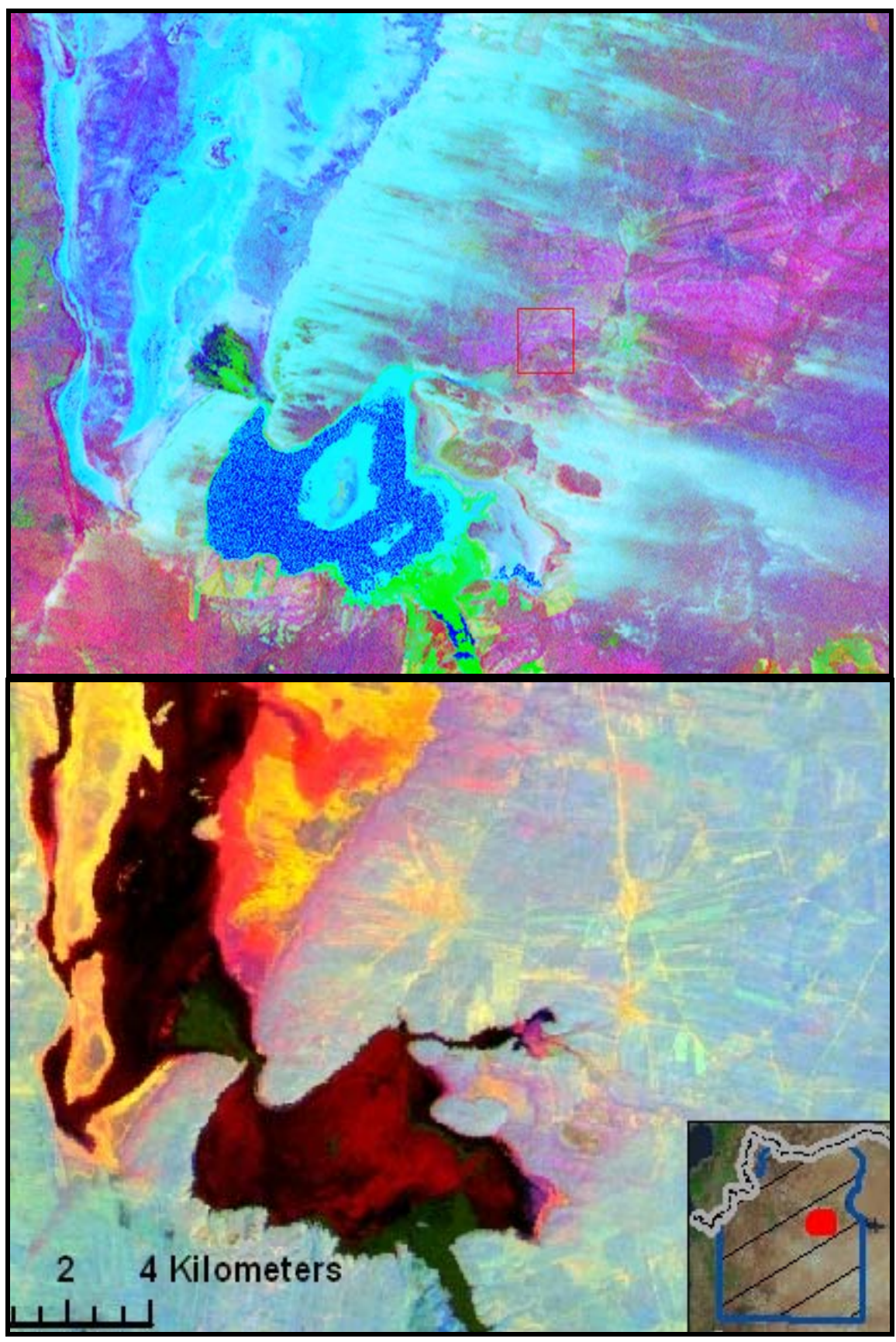

Abb. 5-19: Versalzte Flächen, oben Ratio 3/1, 5/7 und 3/5 \& unten TM 176 (RGB) 
Die Verbreitung von den Schwemmfächern zwischen den abflusslosen Becken und den Palmyra-Ketten erfordert die Suche nach einer Kombination, die eine gute Trennung zwischen den anstehenden Gesteinen und Lockersedimenten ermöglicht. Unter Berücksichtigung, dass dieses Areal wüstenähnlich ist, womit die Reflektion sehr hoch sein kann, wurde auch hier das Ratioverfahren verwendet. Dadurch konnte der Einfluss des Lichteffekts unterdrückt werden. Ein sehr gutes Resultat ist durch die RGB-Kombination der Ratiobilder 3/5, 3/1 und 5/7 (Abb. 5-20) oder 3/1, 5/7 und 3/5 (5-21) gelungen. Dabei wurden auch die lithologischen Unterschiede in den einzelnen Formationen teilweise hervorgehoben. Durch diese Kombination konnten sogar die aktiven Schwemmhalden am Fuß der Palmyra-Ketten erkannt werden (Abb. 5-20).

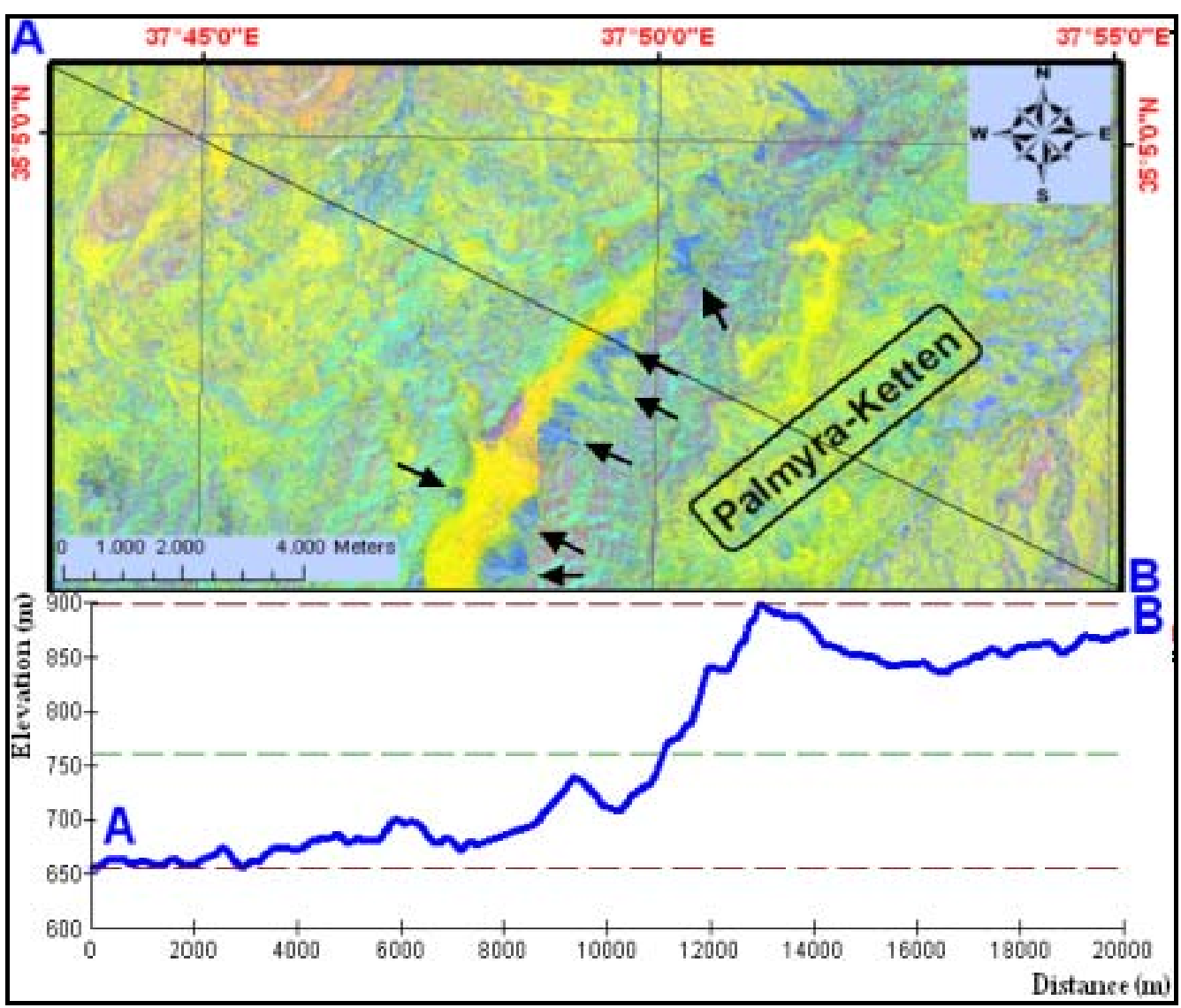

Abb. 5-20: Ratiokombination 3/5, 3/1 und 5/7 (RGB). Die Schwemmhalden (Pfeile) und aktiven Erosionsrinnen sind blau dargestellt 


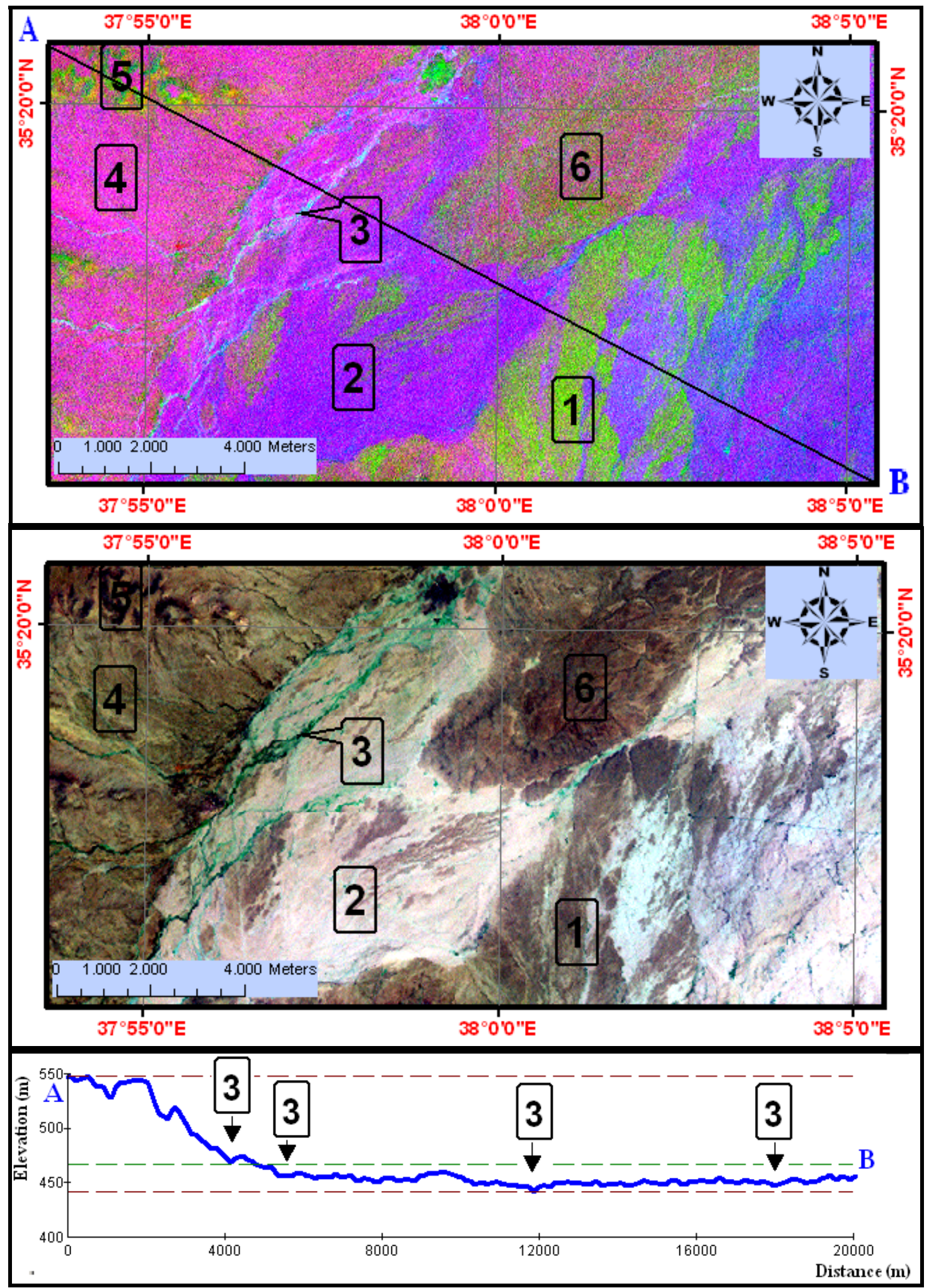

Abb. 5-21: Schwemmfächerverbreitung im Südost des Untersuchungsgebiet Ratiokanalkombination 3/1,5/7 und 3/5 (oben) verglichen mit der ETM+- Kanalkombination 741 (unten) und das topographische Profil (AB).

1- Alte Schwemmfächer (Spätpleistozän), 2- Neue Schwemmfächer (Holozän), 3- Aktive Erosionsrinne, 4- kreidiger bis toniger Kalkstein (Eozän), 5- Kalkstein (Oligozän), 6- Kalkstein (Pliozän). 
Die Lavafelder lassen sich wie bei der geomorphometrischen Auswertung deutlich auf Satellitenbildern kennzeichnen. Es bietet sich hier meistens ein starker Kontrast zwischen den von dem Basalt abgedeckten Felder und ihrer Umgebung sowohl im Westen, wo der Basalt den Kalkstein abdeckt (Abb. 5-22), als auch in den inneren Bereichen, wo der unter dem Basalt liegenden Mergel erodiert ist und lässt die Basaltdecken steil in Schichtstufenform ragen (Abb. 5-16).

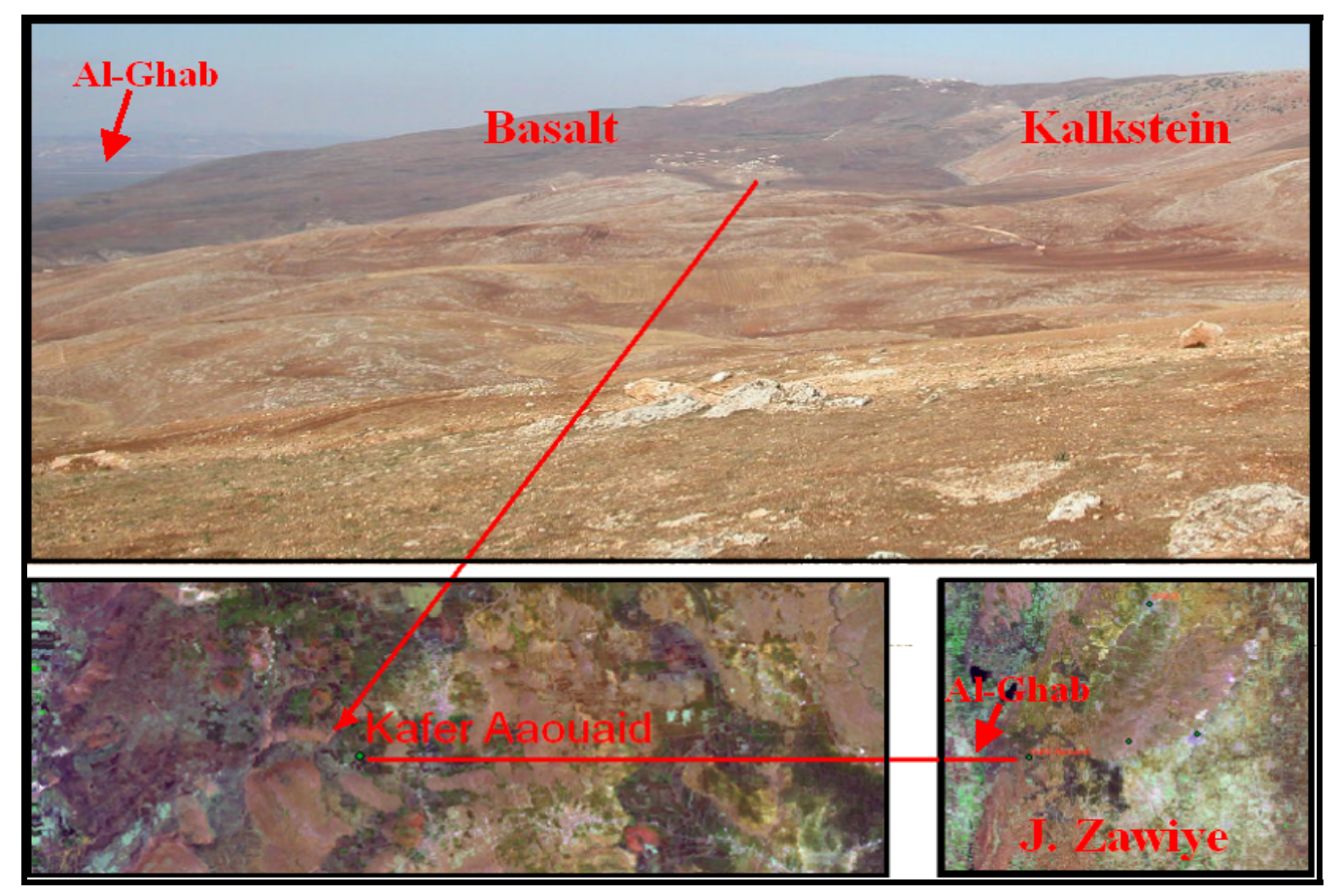

Abb. 5-22: Deutlicher Kontrast zwischen dem Basalt und Kalkstein Landsat (741)

Die Beobachtungen der Basaltfelder lassen feststellen, dass die Verwitterung bis heute nur recht unvollkommene Arbeit innerhalb der Lavadecken leisten konnte, und dass sie meistens öde steinübersäte Flächen Pavement bilden. Doch innerhalb dieser Decken sind Verwitterungsprodukte in Mulden, Wannen oder sanft eingetieften Täler zusammengeschwemmt (Abb. 5-23), und dehnen sich innerhalb der Lavafelder mit einem geringen Kontrast zu dem Basalt aus. Dieser Kontrastmangel wird durch die Kalkkrusten sowie einer dünnen Auflage von Böden und Verwitterungsprodukten verursacht (Abb. 5-23). Deswegen wurden verschiedene Verfahren durchgeführt, um den Kontrast dieser Flächen bei der Kennzeichnung einzuschärfen (Abb. 5-24). Dadurch konnten Verwitterungsprodukte auf den Satellitenbildern charakterisiert werden. Nach ihrer Textur in den Satellitenbildern bestehen sie aus feinen Materialen. Diese wurde während der Geländearbeit in der Region bestätigt. 


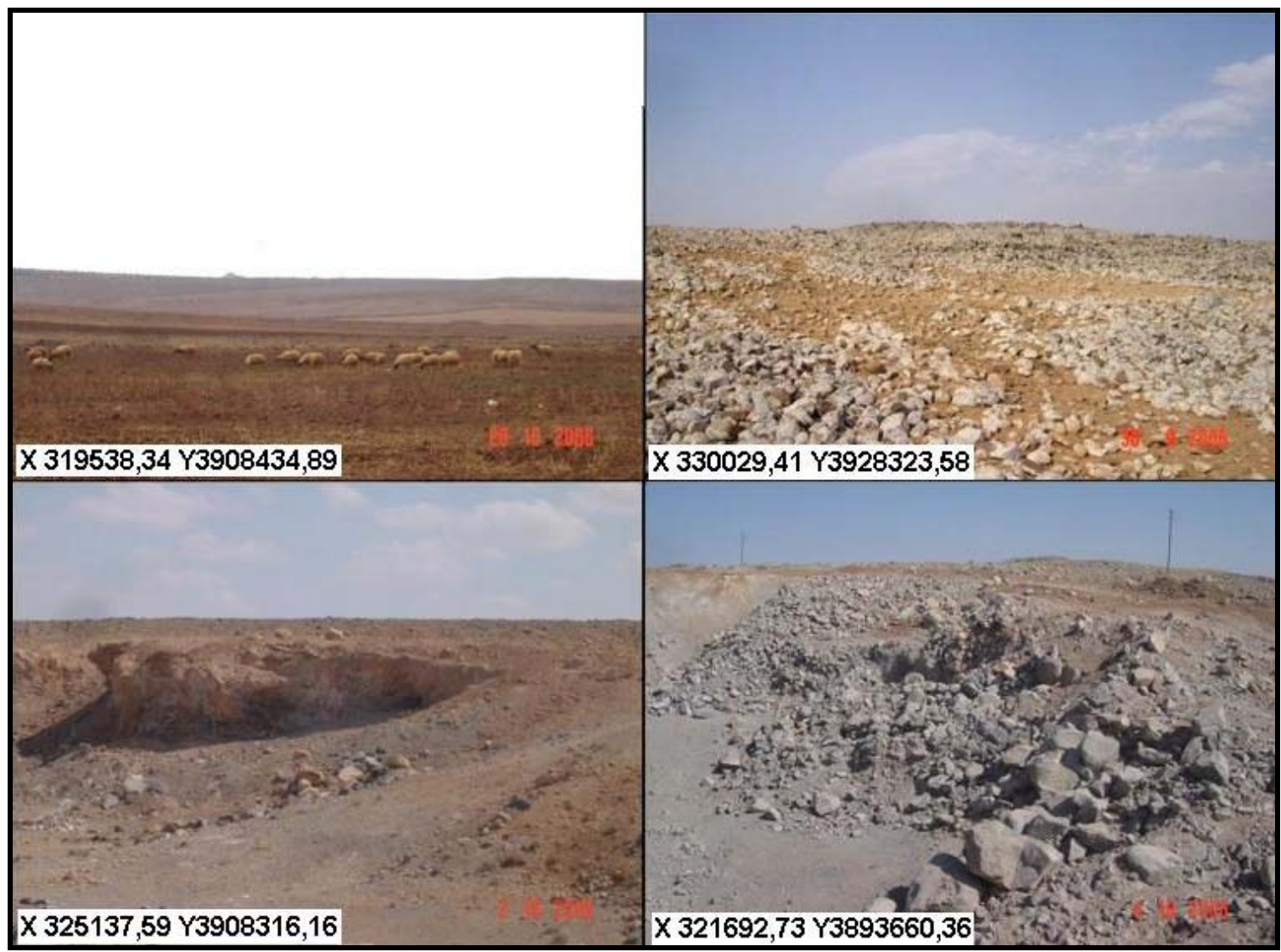

Abb. 5-23: Oben (links Wadi innerhalb von dem Basalt. rechts Basalt-Pavement). Unten (links Basalt-Kalkkrusten. rechts Basalt-Bodenauflage)

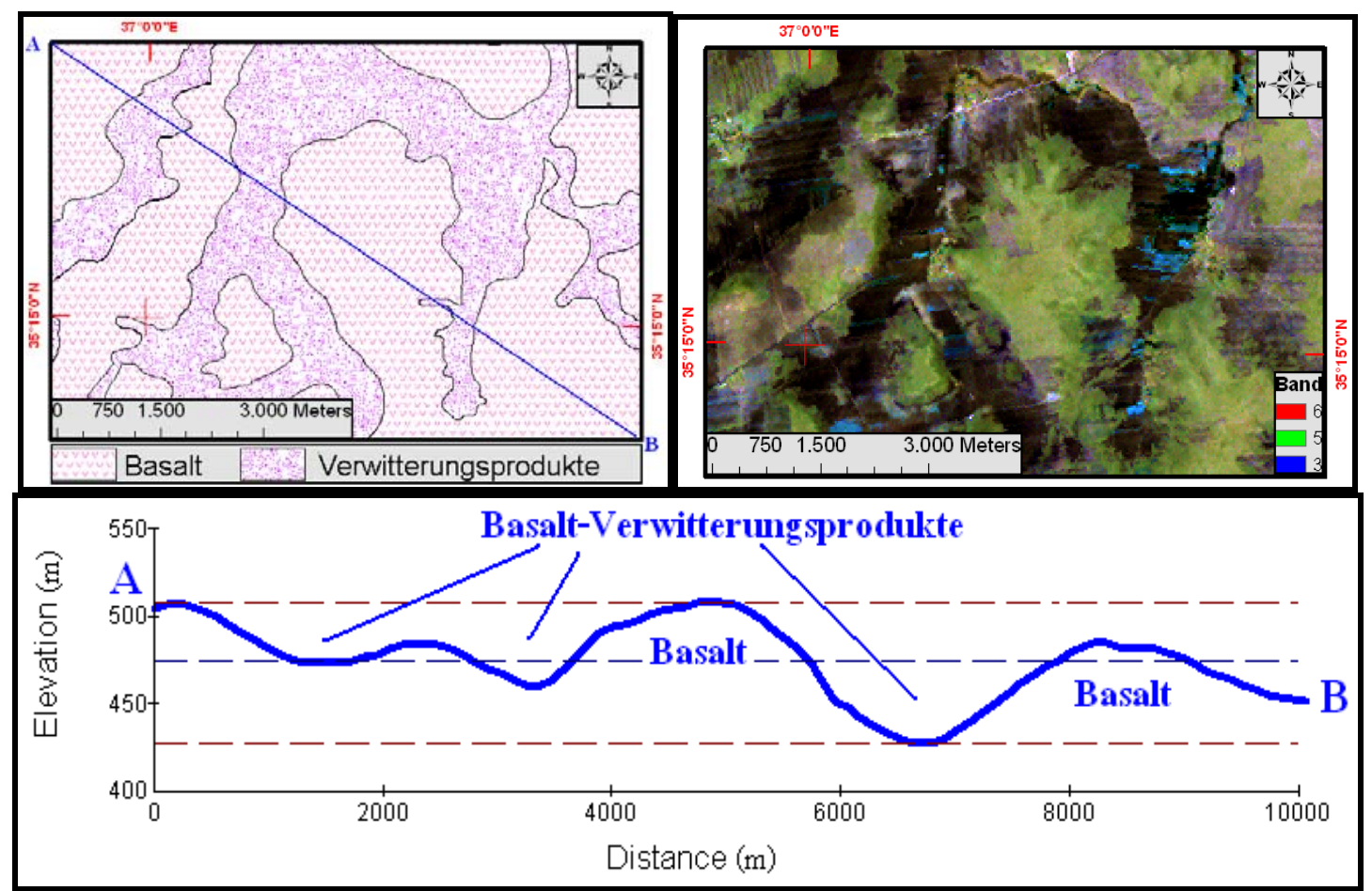

Abb. 5-24: Geomorphologische Kartierung am Beispiel vom Hama-Basalt und Landsat 653 (RGB) 
Die Kalksteinplateaus sind allgemein als nackte öde Landschaft zu bezeichnen. Trotz ihrer geringen Reliefenergie wurden die Verwitterungsprodukte hier abgespült. In diesen Bereichen ist die Verbreitung von fast allen Karst-Lösungsformen (Karren, Dolinen, Uvalas, Poljen, Höhlen, Karstschlote usw.), die nach RoTHER (1993) zum Mediterranen zählen, zu erwarten. Doch durch die Auswertung der vorhandenen Satellitenbilder lassen sich wegen der relativ kleinen Auflösung nur einige Karstformen erkennen. Die typische Karstoberflächenform „Doline“ ist nicht immer direkt aus Satellitenbilder festzustellen. Indirekt kann die Auswertung von den Klüften (Lineamenten) vor einer Geländearbeit verwendet werden, um in Bereichen mit strukturell bedingten Dolinen vorauszusetzen. Dieser Fall wurde auf dem Plateau von Missyaf aufgezeichnet. In der VorortBeobachtung konnten in einem Tal 14 linear verteilte Dolinen gezählt werden. Eine Doline war neu gebildet und hatte eine Größe von etwa $2 * 2$ und eine Tiefe von $3 \mathrm{~m}$.

Langgestreckte oder zusammengewachsene Dolinen können als Uvalas bezeichnet werden. Formen mit einer Größe von mehreren hundert Quadratkilometern werden als Poljen zusammengefasst. Diese Formen sind die Charakterformen des Karsts, die sich gut auf den Satellitenbildern von dem betrachteten Gebiet unterscheiden lassen. Sie sind überall in dem Gebiet mit mehreren Größenordnungen beobachtet worden. Im Westen bilden die Poljen in der öden Karstlandschaft einen guten Kontrast aus, in denen ein bemerkbarer Ackerbau zu beobachten ist (Abb. 5-25). Mit der Verwendung des thermalen Kanal TM6 konnten sogar die frischen Verkarstungsflächen unterschieden werden, wobei diese Flächen hellgelb dargestellt wurden (Abb. 5-25).

Das Georelief im Vorland der Palmyra Ketten, die keine jungen tektonischen Erhebungen erfuhren, ist durch Schichtstufen belebt. Auf Satellitenbilder lassen sich diese Schichtstufen ermitteln. Die Anwendung von dem morphologischen Filter (z. B. Sobel) in dem fast vegetationsfreien Bereich lässt die Schichtkanten bzw. die geologischen Strukturen ausdrücklich dargestellt werden (Abb. 5-26). Für die Kartierung solcher geomorphologischer Feinheiten (Schichtstufen und Schichtkämme) spielte der panchromatische Kanal (15m Auflösung) von Landsat ETM+ eine große Rolle. Durch die Bildfusion dieses Kanals mit den anderen Kanalkombinationen von Landsat konnten die Bildelemente von $30 * 30 \mathrm{~m}$ auf $15 \mathrm{~m}$ verkleinert werden, womit die Erkennbarkeit morphologischer Details besonders verbessert wurde (Abb. 5-27). 


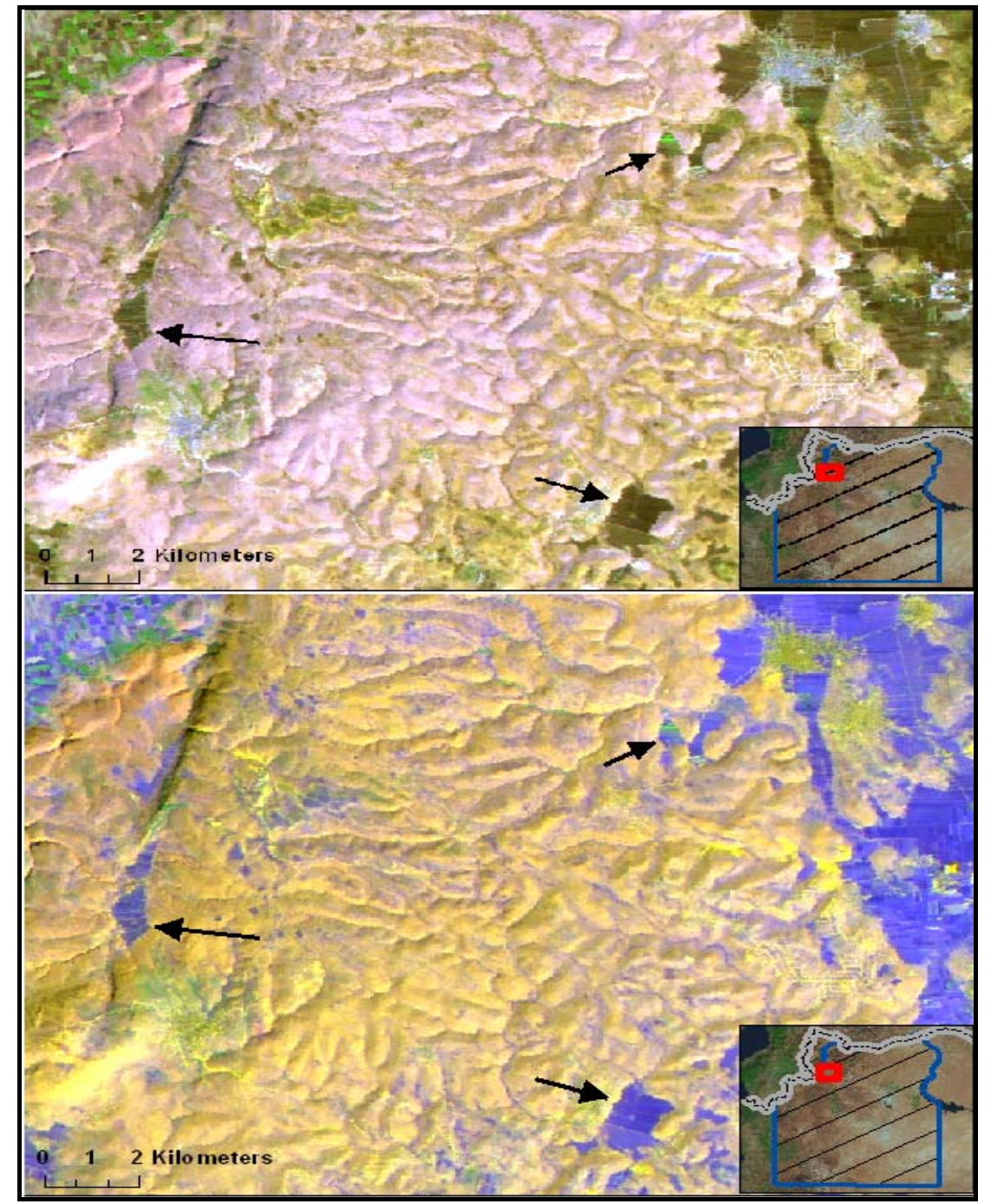

Abb. 5-25: Poljen und Uvalas (Pfeilen) W-Aleppo (oben ETM+ 541, unten TM 546)

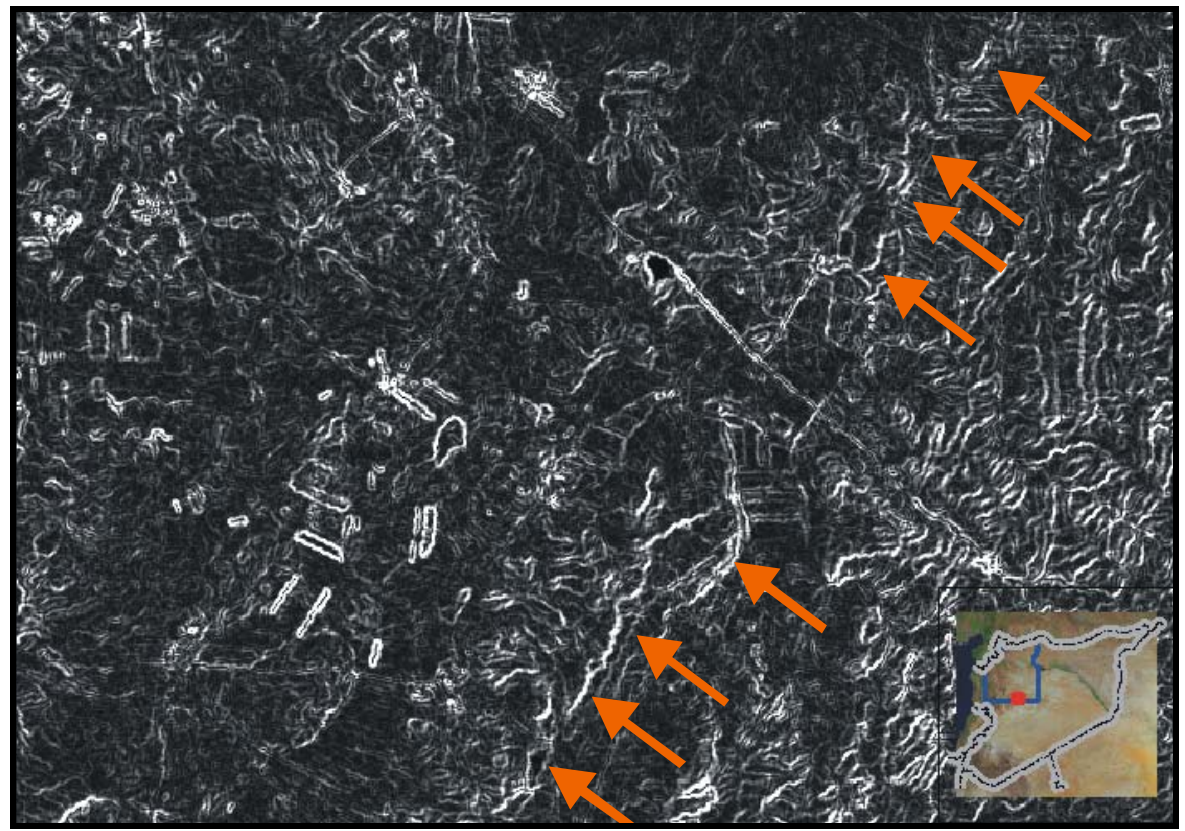

Abb. 5-26: Kantenverschärfung durch die Anwendung von dem Sobel Filter 


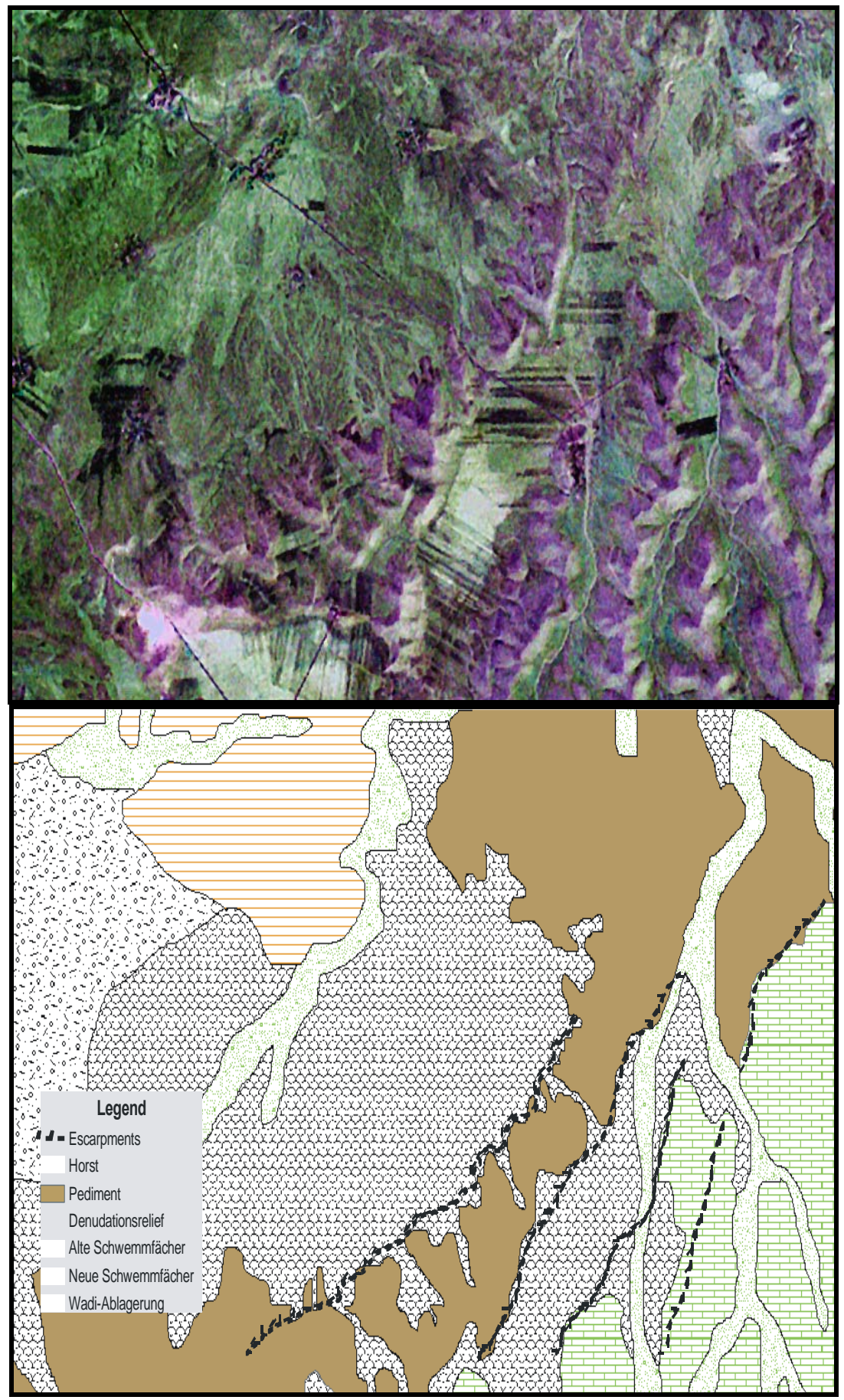

Abb. 5-27: HSV-Fusion des ETM+ (741+8) \& die geomorphologische Auswertung 
- 94 - 
Ka p i t e 16

Morphostrukturelle Darstellungund A n a lyse 


\section{Morphostrukturelle Darstellung und Analyse}

Der Begriff der Morphostruktur beinhaltet die gesetzmäßig bestehende Einheit von tektonischem Bau (die jungtektonischen oder tiefen Strukturen) und lithologischer Zusammensetzung der Erdkruste einerseits und Gestalt des Georeliefs, seinen Formen, andererseits (DEMEK et al., 1982). Infolgedessen müssen die Georeliefformen eines Untersuchungsgebiets in ihrem Zusammenhang zur tektonischen Gesamtstruktur des Gebietes studiert werden. Dabei sollte unterschieden werden zwischen s. z. aktiven und passiven Morphostrukturen. Bei der ersten Form (aktiv) handelt es sich um die Einheit jung tektonischer Strukturen und Dynamik mit dem zugleich endogen und exogen geformten Relief, welche sich am Rand des Untersuchungsgebiets im Bereich des DSFS befinden. Die zweite Form (passiv) repräsentiert die Einheit zwischen dem Relief und tektonisch-lithologischen Verhältnissen, die als Ergebnis älterer Prozesse der Erdkrustenprägung charakteristische Reliefformung in den inneren Bereichen des Untersuchungsgebiets veranlassen.

Die morphostrukturelle Analyse der Landformen beruht auf der Untersuchung der regelhaften Beziehungen, die zwischen dem Georelief und den jung tektonischen oder tiefen Strukturen bestehen. Dieser Analyse stehen die Fernerkundungsmethoden zur Verfügung, die schnelle und verhältnismäßig preiswerte Informationen geben können. Die Fernerkundungsaufnahmen können Kenntnisse über die Struktur und die geologischtektonische Geschichte eines großflächigen Gebietes wiedergeben (LIST et al., 1990; Akman \& Tüfekçi, 2004). Ihre Auswertung kann mit den klassischen geologischen Geländeuntersuchungen ergänzt werden, um den Verlauf der Strukturen im Untergrund festzustellen. Das wichtigste Verfahren dabei ist die Analyse der morphologischen Merkmale insbesondere der Lineamente, die in vielen Studien zur Untersuchung der geologischen Strukturen und Tektonik verwendet wurden (HoBBS, 1912; FRISCH, 1997). Diese Anwendung ist $\mathrm{zu}$ einem grundlegenden Werkzeug in den tektonischen Analysen mittels der Luftbilder durch die stereoskopische Auswertung (Photogeologie) und anderen Fernerkundungsdaten geworden (SIEGAL \& GILLESPIE, 1980; SALVI, 1995; DRURY, 2001).

Weiterhin wird die Analyse der topographischen Karten und die Verwendung graphischer und statistischer Verfahren benutzt, welche zurzeit durch Einsatz des digitalen Höhenmodells erleichtert wird. Diese führt zur Entwicklung der tektonischen digitalen Geomorphologie. Dieser Begriff entsteht durch die Integration von drei Bestandteilen: i. die 
Strukturgeologie, ii. die Geomorphologie und iii. die digitale Geländeanalyse (JORDAN et al., 2003 und JorDan, 2003). Ein bedeutendes Ziel dieser Disziplin ist die Gewinnung der morphotektonischen Merkmale von digitalen Höhenmodellen.

Die Interpretation der Landformenmorphologie hinsichtlich der Strukturgeologie ist gut etabliert (Prost, 1994; Burbank \& Anderson, 2001; Keller \& Printer, 2002). Diese Arbeiten haben die Georeliefformen, die auf Verwerfungswirkungen basieren, diskutiert.

Diese Fragestellung hier beruht grundlegend auf der Erfassung der Richtung der linearen Elemente und ihrer Zuordnung zu der Tektonik. Neben der Darstellung der Auswertungsergebnisse soll in diesem Kapitel auch Bezug genommen werden auf den zusammenhängenden tektonischen Baustil.

\subsection{Statistisch morphostrukturelle Analyse}

Die morphostrukturelle Analyse erfuhr eine bedeutsame Entwicklung durch den Einsatz des digitalen Höhenmodells (SIMPSON \& ANDERS, 1992; BYRD, 1994; COLlET et al., 2000) und der Fernerkundungsdaten oder durch die Kombinierung der beiden Daten (FlORINSKY, 1998; ChOROWICZ, 1999). OnORATI et al. (1992) untersuchte die regionale Tektonik in Italien durch die Berechnung der Hangneigung und Exposition. JORDAN et al. (2005) präsentiert eine Prozedur der digitalen tektonischen Geomorphologie, die auf das Extrahieren der morphotektonischen Strukturen zielt. Die geomorphometrischen Parameter vor allem die Exposition und Hangneigung sind meistens im Zusammenhang mit der räumlichen Variabilität der Morphotektonik bzw. der Tektonik und der Lithologie sehr kontrovers diskutierende Einflussgrößen. Somit können sie für die Beschreibung der Morphostrukturen aussagekräftig sein. Demgemäß wurde in dieser Studie die Exposition zuerst berechnet und analysiert, um sie im weiteren Verlauf mit der Hangneigung zu kombinieren.

Die Exposition (Gradient) bezeichnet die Lage eines Hanges in Bezug auf die Einfallsrichtung der Sonnenstrahlen. Die Expositionsrichtung kann zahlenmäßig angegeben werden als Richtung der Flächennormale in Grad, bezogen auf die Nordrichtung (in Alt-Grad $0^{\circ}-360^{\circ}$ ). Auf diese Weise repräsentieren der minimale und maximale Wert die gleiche Exposition (Zirkular-Verteilung). Das heißt, weit auseinander liegende Wertebereiche, wie beispielsweise $5^{\circ}$ und $355^{\circ}$, liegen bezüglich ihrer Exposition sehr nahe beieinander. Die Zuordnung der Exposition erfolgte nach acht Expositionsklassen, die eine Breite von jeweils $45^{\circ}$ aufweisen. 
Im gesamten Untersuchungsgebiet ist eine leichte Dominanz der Exposition nach Nordwesten oder Südosten $(26,98 \%)$ und nach Westen oder Osten $(24.79 \%)$ zu verzeichnen (Abb. 6-1). Demgegenüber sind die Exposition nach Norden oder Süden mit 23.60\% am seltensten vertreten (Tab. 6-1). Mit Berücksichtigung der anderen Georeliefattribute und der geologischen und paläogeographischen Entwicklung des Untersuchungsgebiets kann die statistische Auswertung des o. d. Wertespektrums bei der Georeliefanalyse bedingt verwendet werden, um primäre Aussagen zu treffen. Das Auftreten von theoretisch möglichen Hängen in alle Richtungen kann (aus der strukturellen Ansicht) bestätigen, dass sich die im Kapitel (2.3) erwähnte Tektonik in dem Gebiet widerspiegelt. Folglich ist der hohe Prozentanteil der nach Westen exponierten Hänge, auf die Wirkung des jungen DSFS zurückzuführen.

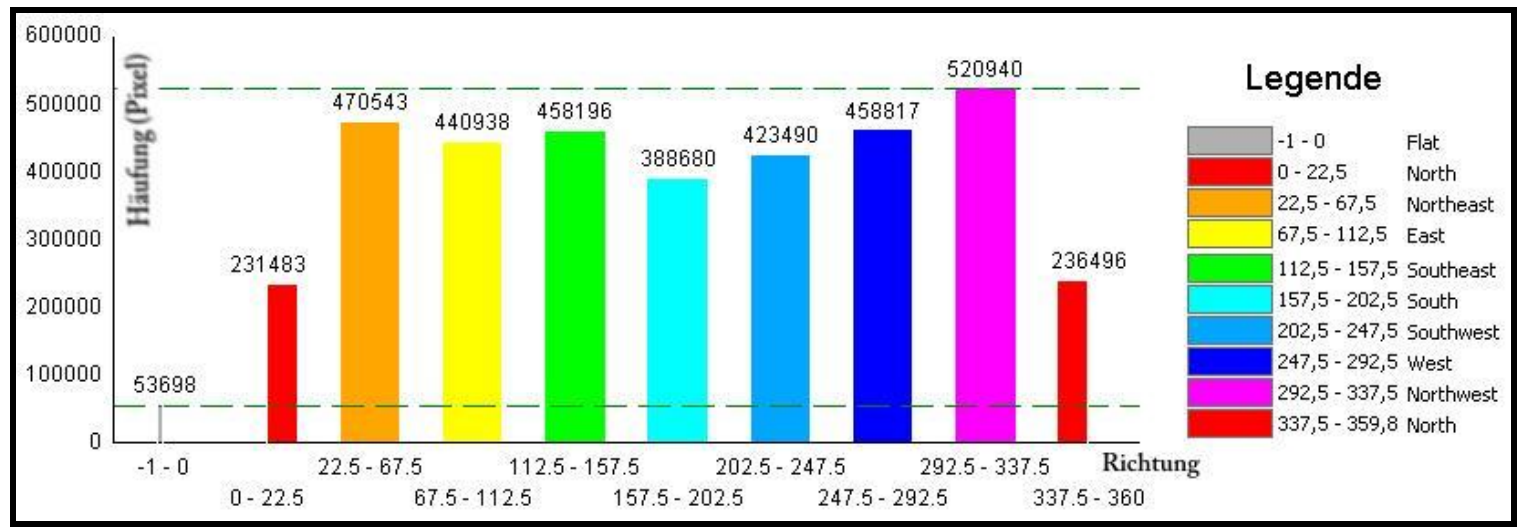

Abb. 6-1: Histogramm der Expositionsverteilung

Tab. 6-1: Expositionshäufigkeit

\begin{tabular}{|c|c|c|c|}
\hline Exposition & $\begin{array}{c}\text { Anzahl } \\
\text { der Pixel }\end{array}$ & Prozent (\%) & Richtung \\
\hline N oder S & 856659 & 23.60 & E-W \\
\hline NE oder SW & 894033 & 24.63 & NW-SE \\
\hline E oder W & 899755 & 24.79 & N-S \\
\hline NW oder SE & 979136 & 26.98 & NE-SW \\
\hline \multicolumn{4}{|c|}{ Das gesamte Untersuchungsgebiet besitzt 3629583 Pixel. } \\
\hline
\end{tabular}

Durch Einsatz des Rasterrechners (Raster Calculatur) konnte jede Richtung mit den Hangneigungsrasterdaten überschnitten werden, wobei der Hangneigungswert über $1^{\circ}$ kalkuliert wurde. Die Landoberfläche mit Hangneigung weniger als $1^{\circ}$ wurde als flaches Land eingestuft. Die Ergebnisse wurden auch in ArcGIS mit den Funktionen Boundary 
Clean und Majority gefiltert, um die vereinzelten Pixel (nachbarlose Pixel) zu beseitigen (Abb. 6-2).

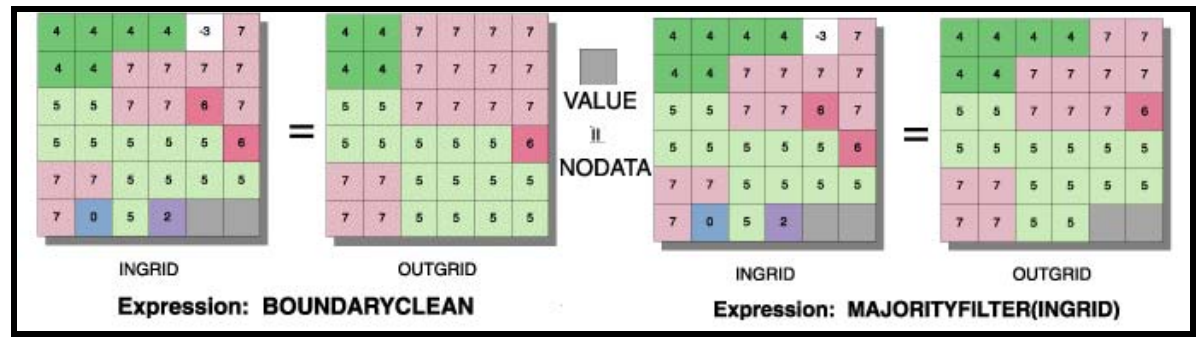

Abb. 6-2: Arithmetische Ausdrücke der verwendeten Filter.

Der Vergleich zwischen den aus geologischen Karten digitalisierten Verwerfungen und den resultierten Rasterdaten (Abb. 6-3) zeigt, dass entlang der bekannten Verwerfungen eine Art von linearen Verteilungen der Pixel mit den gleichen Geländewerten zu beobachten ist. Diese lineare Verteilung ist auch in anderen Bereichen $\mathrm{zu}$ finden, welche als Indikator für lineare morphologische Merkmale verwendet werden kann, insbesondere mit der Kombination anderer Kriterien wie das geschummerte Reliefmodel.

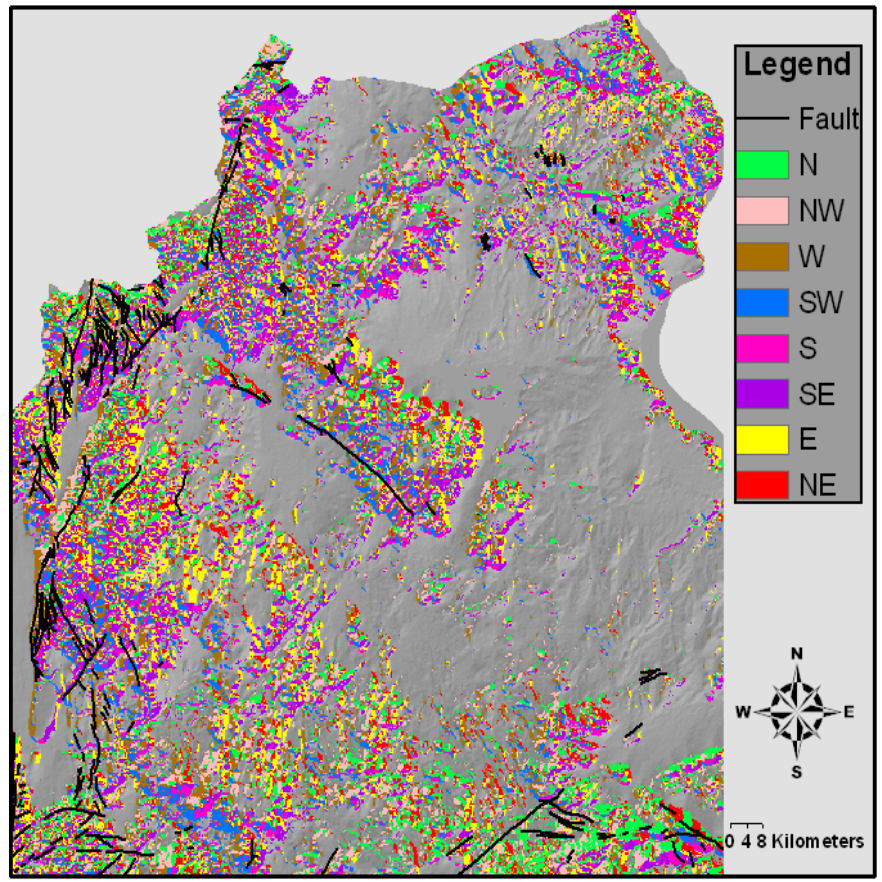

Abb. 6-3: Exposition verschnitten mit Hangneigung (Basis ist das geschumm. Modell)

Mit dieser Beobachtung und mit der Annahme, dass die Exposition für die Beschreibung der Morphostrukturen aussagekräftig sein könnte, kann festgestellt werden, dass die nach Nordost verlaufenden linearen Strukturen dominant sind (Tab. 6-1). Dieser statistische Zusammenhang zwischen der Tektonik und der Exposition wird auch durch die Analyse der Lineamente nachgeprüft. 


\subsection{Visuelle Ermittlung der linearen Merkmale}

Die Auswertung der geomorphologischen Phänomene im Sinne der geologischen Strukturen ist gut von Prost (1994) und Keller \& PinTer (2002) beschrieben. In diesem Rahmen hat die visuelle Interpretation der Morphostrukturen anhand von Lineamenten mit der zunehmenden Verwendbarkeit der Fernerkundungsdaten an Popularität gewonnen. Dieser Einsatz verlangt eine klare Definition des Begriffs Lineamente. O'LEARY et al. (1976) diskutierten den Ursprung und die Verwendung der Begriffe linear, Lineation und Lineamente. Sie haben den Begriff Lineamente in einem wesentlichen geomorphologischen Sinn definiert, wobei ein Lineament als kartierbare lineare bis kurvilineare Eigenschaft einer Oberfläche definiert ist, deren Teile in einer geraden oder leicht gebogenen Beziehung zueinander stehen. Nach CLARK \& WILSON (1994) sind gerade lineare Elemente auf der Erdoberfläche erkennbar, die geologische und/oder geomorphologische Phänomene widerspiegeln. In den geomorphometrischen Analysen können die linearen Phänomene ausschließlich eine geometrische Herkunft haben, womit sie eine Änderung in der Geländehöhe wie ein Tal, eine Kammlinie oder Neigungsbrechung darstellen. Im Sinne der digitalen Modellierung ist ein Lineament eine ununterbrochene Reihe von Bildelementen (Pixel), die ähnliche Geländewerte haben (KOIKE et al., 1998). KRONBERG (1977) bezeichnet in der Luft- und Satellitenbildinterpretation die Schnittlinien mit der Erdoberfläche, die in Abhängigkeit vom Einfallen der Kluft- und Störungsflächen kurvilinear, unregelmäßig oder - bei steilstehenden Störungen - geradlinig verlaufen, ohne Genesebezug neutral als „Photolineationen“. Nach PROST (1994) können Satellitenbildinterpretationen zur Erfassung bruchtektonischer Großstrukturen vollkommenere und bessere Daten liefern als Geländeuntersuchungen.

In dem folgenden Kapitel werden zunächst die Kriterien wie die Topographie, Morphologie und Drainage durch den Einsatz des digitalen Höhenmodells verwendet, um die Lineamente festzustellen. Die Ergebnisse werden mit den Satellitenbildern kombiniert und überprüft, dabei werden auch die Kriterien der Photogeologie/PhotoGeomorphologie eingesetzt. 


\subsubsection{Ermittlung von Lineamenten durch Einsatz des DHM}

Die Anwendung von digitalen Höhenmodellen hat sich durch zahlreiche Studien als eine gute Methode etabliert, um die linearen und/oder morphotektonischen Georeliefformen und strukturelle Grenzen zu verfolgen bzw. zu kartieren sowie räumliche Verhältnisse der Strukturen aufzudecken und zu analysieren (z.B., MURPHY, 1993; WDOWINSKI \& ZILBERMAN, 1997; GOLDSWORTHY \& JACKSON, 2000; GANAS et al., 2001; VYSOTSKY et al., 2002 \& JORDAN, 2003). Dabei wurde das geschummerte Reliefmodell (shaded relief model) allein oder kombiniert mit anderen geomorphometrischen Parametern wie Hangneigung, Krümmung, Fließlänge und anderen Aspekten des digitalen Höhenmodells verwendet. Das geschummerte Höhenmodell wurde für die Visualisierung der linearen morphologischen Merkmale in Glazialgebieten (LIDMAR-BERGSTRÖM et al., 1991) und entlang bekannter Verwerfungen erfolgreich eingesetzt (LEEDER \& JACKSON, 1993; PIKE, 2000; GANAS et al., 2001; GANAS, 2002 \& OGUCHI et al., 2003). Das Nützlichste bei diesem Modell ist die eingängige Verwendung einer idealisierten Lichtquelle für die Diagnose der Morphologie, die die Betonung der strukturellen Elemente oder die Unterbrechung in der Topographie ermöglicht (PIKE, 1992). Dies geschieht in Abhängigkeit von der gewählten Sonnenhöhe und Richtung. Ein Nachteil dieses Modells liegt darin, dass einige lineare Merkmale bei einem bestimmten Beleuchtungsazimut wenig visualisierbar sind (Azimuth-Biasing). Das erfordert eine Änderung des Beleuchtungsazimuts, um die Visualisierung zu verbessern (LIDMAR-BERGSTRÖM et al., 1991). Auf der anderen Seite bezieht sich der Vorteil dieser Methode auf diese Funktion (ONORATI et al., 1992), wodurch eine Untersuchung der Strukturen mit einem benutzerspezifischen Azimut und bestimmter Sonnenhöhe möglich ist (SMITH \& CLARK, 2005), d. h. die morphologischen Strukturen in einem Gebiet können von allen Himmelsrichtungen ausgewertet werden (Abb. 6-4).

Das betrachtete Gebiet ist durch ein einfaches Georelief charakterisiert, das im Zentrum sehr flach ist. Aus diesem Grund war eine vertikale Exaggeration (5*) notwendig, um die Identifikation der morphologischen Elemente zu verbessern. Unter Berücksichtigung, dass die geologischen Strukturen eine Tendenz nach NE-SW, NW-SE und N-S haben, wurden verschiedene Modelle mit einer Beleuchtung von SE, SW, NE und NW erstellt. Dabei war die Sonnenhöhe konstant mit einem Wert von $45^{\circ}$. 


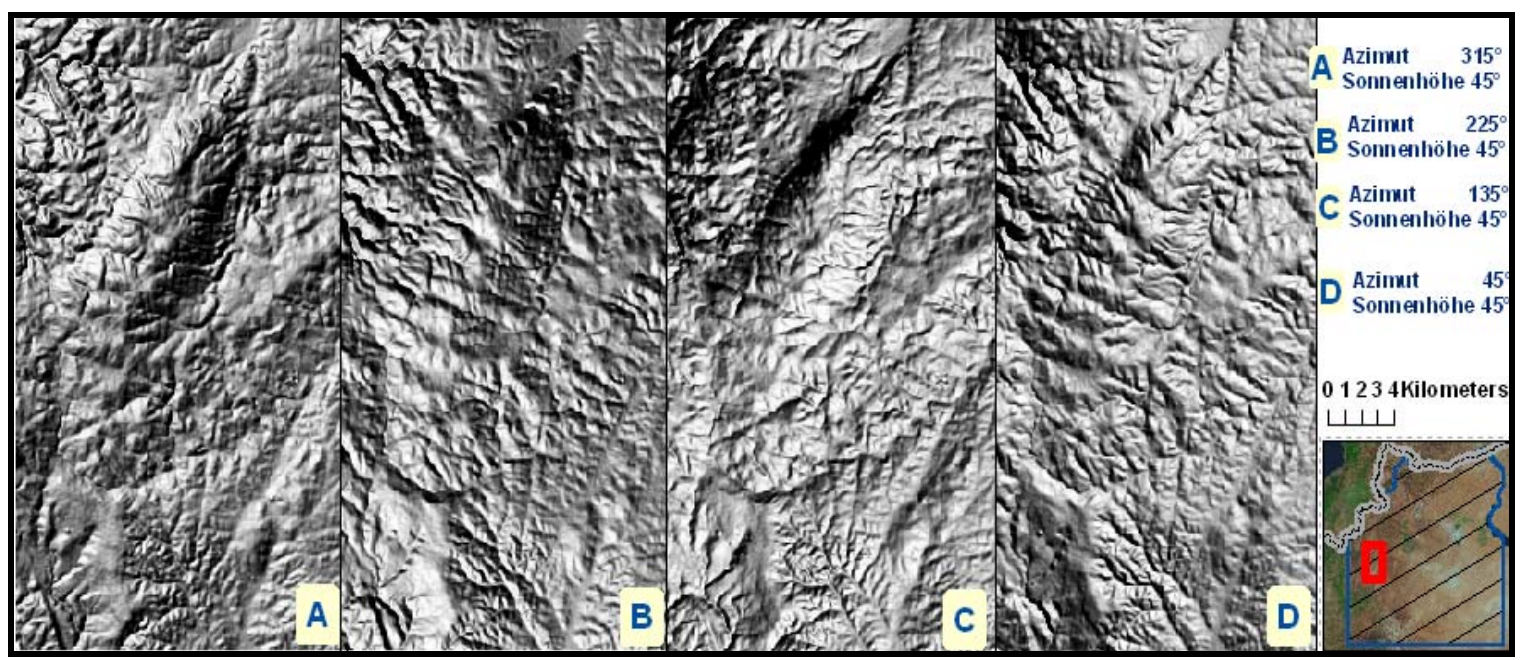

Abb. 6-4: Einfluss der Azimutänderung auf dem geschummerten Reliefmodel

Bei der Auswertung der linearen Strukturen wurden zunächst die bereits auf geologischen Karten festgestellten Verwerfungen verfolgt. Danach wurden die anderen linearen morphologischen Elemente, die möglicherweise eine strukturelle Bedeutung haben, ermittelt (Abb. 6-5).

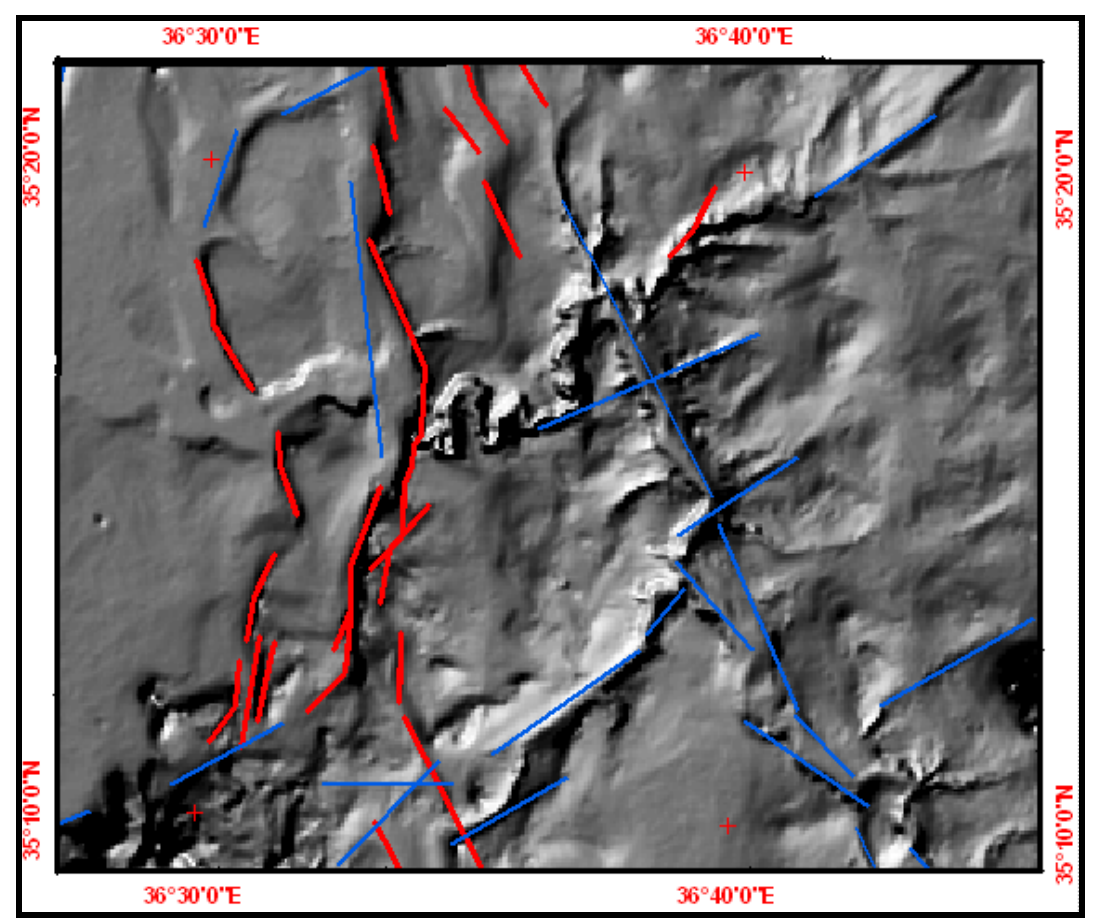

Abb. 6-5: Geschummertes Reliefmodell (Azimut 135'), Verwerfungen (Rot) und lineare Phänomenen (Blau) 
Das DHM wurde für die Extrahierung des Entwässerungsnetzes verwendet. Diese Methode hat gegenüber der Digitalisierung des Entwässerungsnetzes von den topographischen Karten für die regionale Analyse den Vorteil, dass es bei kombinierten Analysen mit dem DHM zu 100 \% kompatibel ist, da es aus ihm generiert wird. Dadurch sind die linearen Morphostrukturen, die auf Basis der Analyse des Entwässerungsnetzes beruhen, viel einfacher zu interpretieren bzw. mit anderen Auswertungen genauer zu kombinieren (Abb. 6-6).

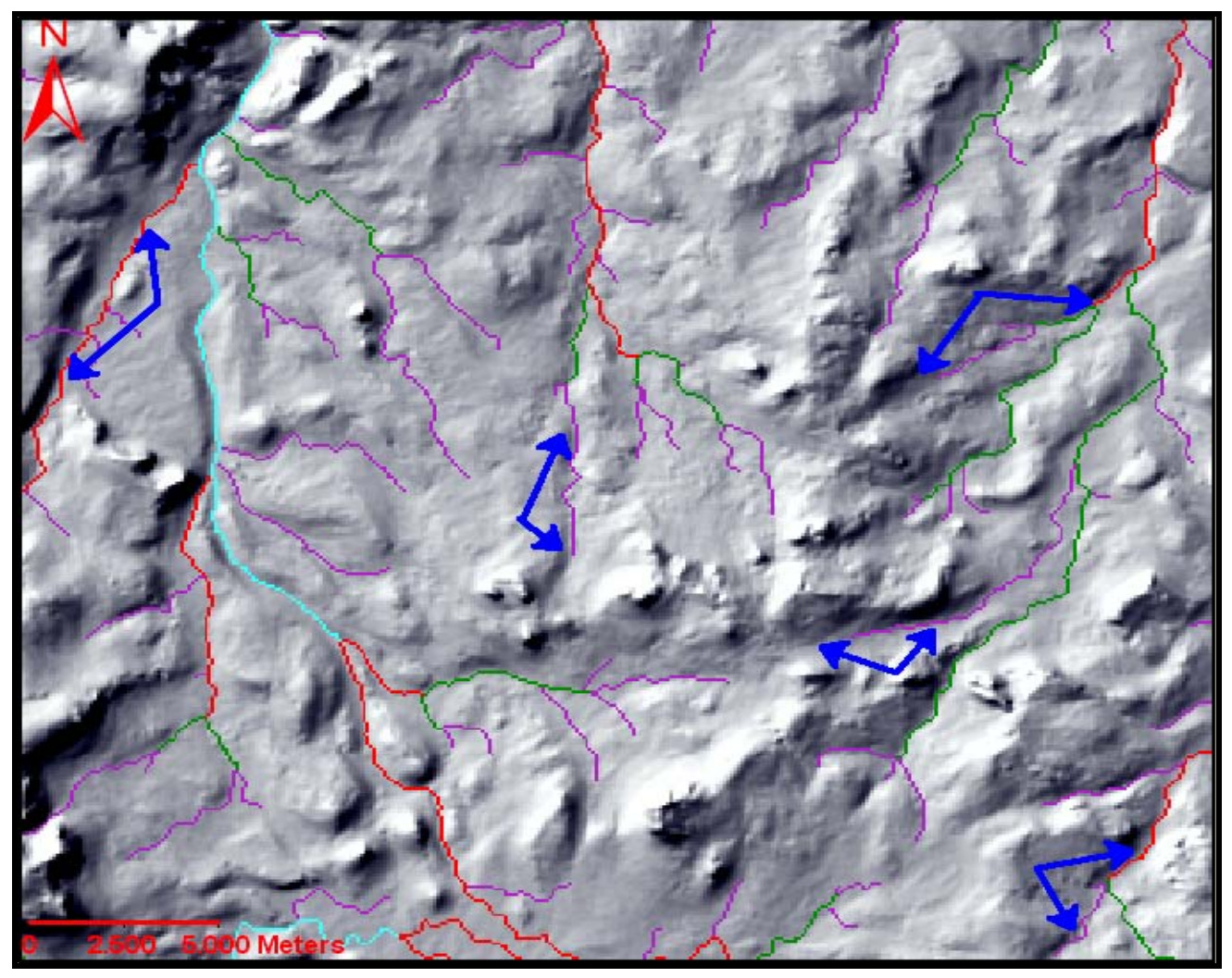

Abb. 6-6: Das erzeugte Entwässerungsnetz (Pfeile zeigen einige lineare Merkmale) 


\subsubsection{Ermittlung von Lineamenten auf den Satellitenbildern}

Der visuellen Kennzeichnung der Lineamente in Satellitenbildern stehen nach zahlreichen Studien verschiedene Kriterien, die sich auf die Bildeigenschaften oder auf die in den Bildern auffälligen morphologischen Merkmale beziehen, zur Verfügung. Zu den Ersten gehört die Bildfarbe, -beschaffenheit, -ton oder -muster. Zu den Zweiten zählen die eckigen Brüche in den Entwässerungsmustern, gerade Bachsegmente, lineare Klippen, Eskarpments etc. (LATTMAN \& PARIZEK, 1964). Alle diese Eigenschaften wurden während der Auswertung berücksichtigt.

Für die Satellitenbildinterpretation standen mehrere Satellitenbilder zur Verfügung (Tab. 3-2). Die Bilder wurden in unterschiedlichen Zeiten mit unterschiedlicher Sonnenlage bzw. unterschiedlichen klimatischen Bedingungen aufgenommen. Diese hat bedeutende Vorteile, u. a. die mögliche Auswertung eines Bereichs bei unterschiedlichen Schattensituationen, wobei die potentielle Verbergung der morphologischen Strukturen unter Schatten minimiert werden kann. Hierbei waren die Funktionalitäten des ArcGIS Programms, mehrere Bilder verschiedener Szenen bzw. Kombinationen übereinander zu legen bzw. nacheinander aufzurufen, für die Analyse sehr produktiv.

Die Ermittlung der Lineamente und der linearen Strukturen in mehreren Bereichen des Gebiets war nicht einfach und zum Teil kompliziert. Dazu tragen zum einen die sehr schlechten Aufschlussverhältnisse bei, zum anderen die speziellen lithofaziellen Gegebenheiten des Gebietes. Es treten insbesondere die gleichen Typen von Vulkaniten und Sedimenten innerhalb der von der Kreide bis ins jüngste Tertiär reichenden Schichtfolge auf. Hinzu kommen häufige Verzahnungen oder Abdeckungen der vulkanischen und sedimentären Einheiten. Dadurch wird es oft sehr schwer, lithofazielle bzw. stratigraphische Leithorizonte zu fixieren und über größere Entfernungen zu verfolgen. Infolge dessen war bei dieser Arbeit wie in der photogeologischen Kartierung die Ermittlung der Lineamente auf den o. g. indirekten Kriterien angewiesen. Hierbei wurden mehrere Fernerkundungsverfahren verwendet, um die Interpretation $\mathrm{zu}$ erleichtern. Die LinearStreckung (2\%) wurde lokal eingesetzt. Das Sobel (Abb. 5-26) und High-Pass Filterverfahren stellt sich als sehr nützlich heraus, insbesondere nach der Bildfusion. Die Methode des Richtungsfilters wurde in mehrere Richtungen durchgeführt, wodurch die Lineamente in mehreren Teilen deutlich hervorgehoben worden konnten. In dem gleichen Maße war das PCA-Verfahren (Principal Component Analysis) sehr hilfreich (Abb. 6-7). 
Neben den farbigen Satellitenbildern, die bei den geomorphologischen/lithologischen Auswertungen vorteilhaft waren, wurden bei der Auswertung von den Lineamenten auch Schwarz-Weiß-Aufnahmen verwendet. Die Gründe für diese Anwendung sind einerseits ein möglicher schärferer Kontrast und andererseits eine höhere Konzentration des Analysten, da Farben ablenken können.

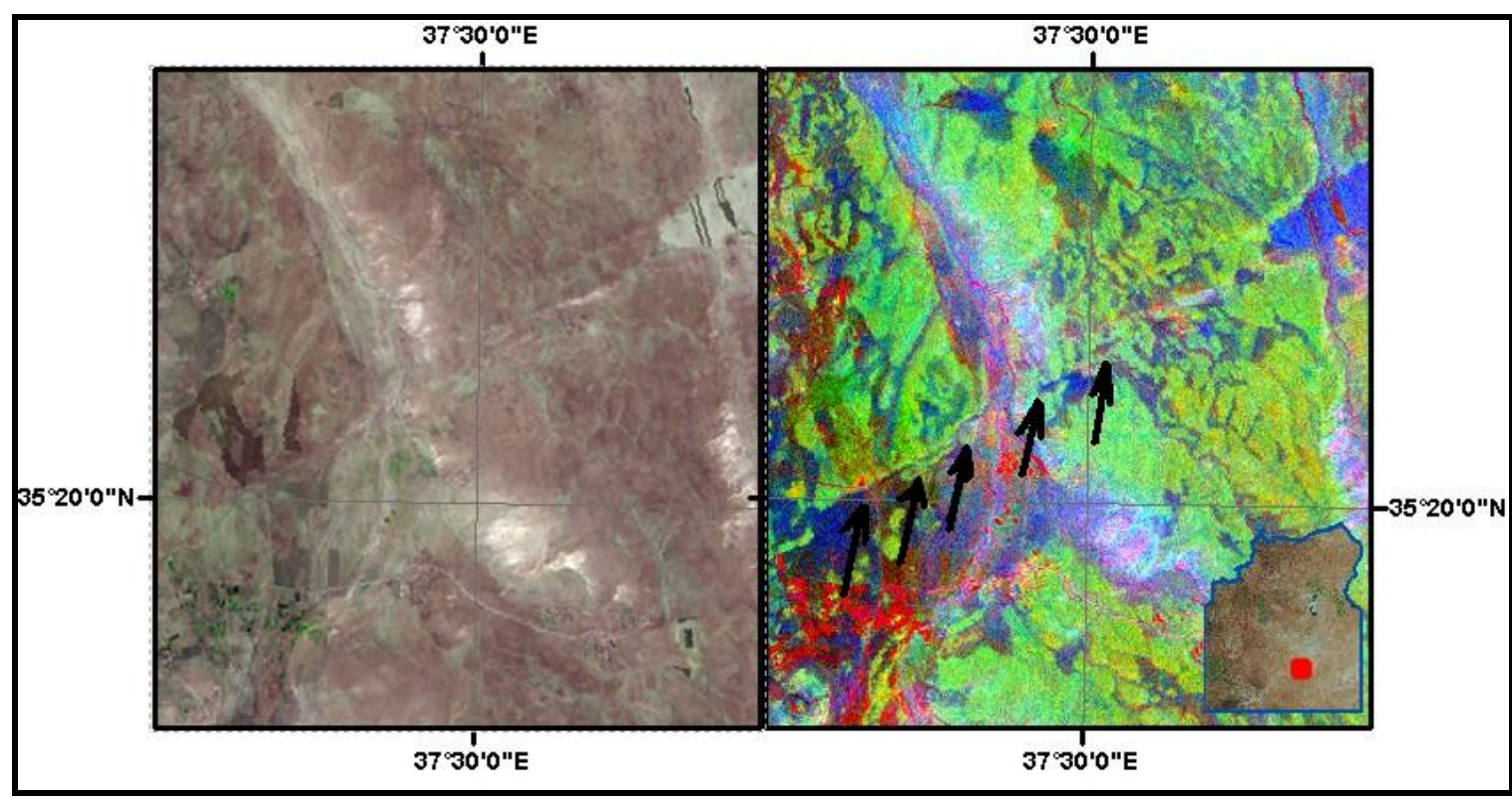

Abb. 6-7: Verbesserung der visuellen Interpretation mittels PCA-Verfahren; Links Landsat 542 (RGB) und rechts PC4, PC3 und PC2 (RGB)

In Bereichen mit einer Quartärabdeckung wurden als Lineamente die dunklen oder hellen Linien im Boden und die Ausrichtungen der Vegetation interpretiert. Diese Zonen lassen sich nach Ansicht von ROLAND (1973) erklären. Nach seiner Meinung sind die Klüfte und Störungen Schwächezonen, in denen die Verwitterung verstärkt ansetzen kann. Die starke Wirkung der Erosion durch fließendes Wasser in Kluftbereichen bildet Entwässerungssysteme unterschiedlicher Größe und Form. Längere Verläufe der Wadis können deshalb indirekt als Lineamente interpretiert werden. In Bezug darauf ließen die Satellitenbildbetrachtungen vermuten, dass sich zumindest die großen parallel bis subparallel oder rechtwinklig zueinander verlaufenden Wadis störungsorientiert gebildet haben. 
Für die Auswertung der strukturell bedingten Wadis wurde auch das Ratioverfahren verwendet. Eine hervorragende Hervorhebung des Entwässerungsnetzes wurde durch die Ratio-Kombination 7/5, 5/4 und 3/1 erreicht (Abb. 6-8). Entscheidend hierfür war, dass der Einfluss der Lichteffekte unterdrückt werden konnte.

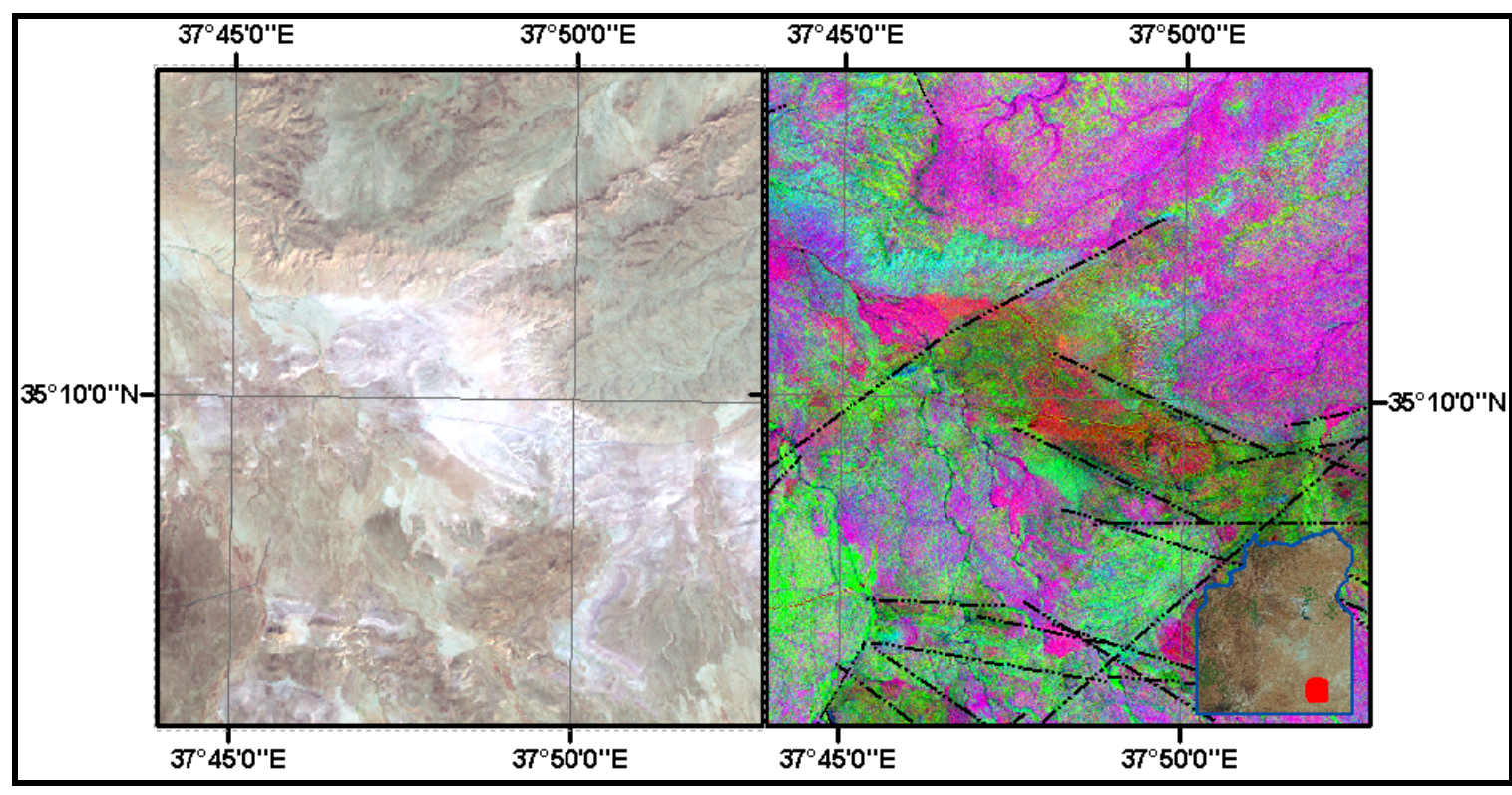

Abb. 6-8: Vergleich zwischen der Kanalkombination 542 und der Ratiobildkombination $7 / 5,5 / 4$ und $3 / 1$

\subsection{Richtungsverteilung der Lineamenten}

Die ausgewerteten Lineamente sind im zweidimensionalen Koordinatesystem (XY) ohne Angaben der Richtung gespeichert. Die für die statistische Auswertung der Daten notwendigen Streichrichtungen wurden durch die Anwendung der Extension Easy Calculate in ArcGIS ermittelt. Diese Richtungsdaten wurden als Datei im Textformat exportiert und konnten so in dem Tektonik-Programm StereoNet weiterverarbeitet werden. Die Ergebnisse der Auswertungen sind in Form von bidirektionalen Rosendiagrammen dargestellt (Abb. 6-9 und Abb. 6-10). Durch diese Darstellung ergeben sich im Untersuchungsgebiet drei signifikante Tendenzen. Die dominierende Tendenz der Lineamente ist die \pm NE-SW Richtung. Die Zweite ist \pm NW-SE und die Dritte \pm N-S gerichtet. 


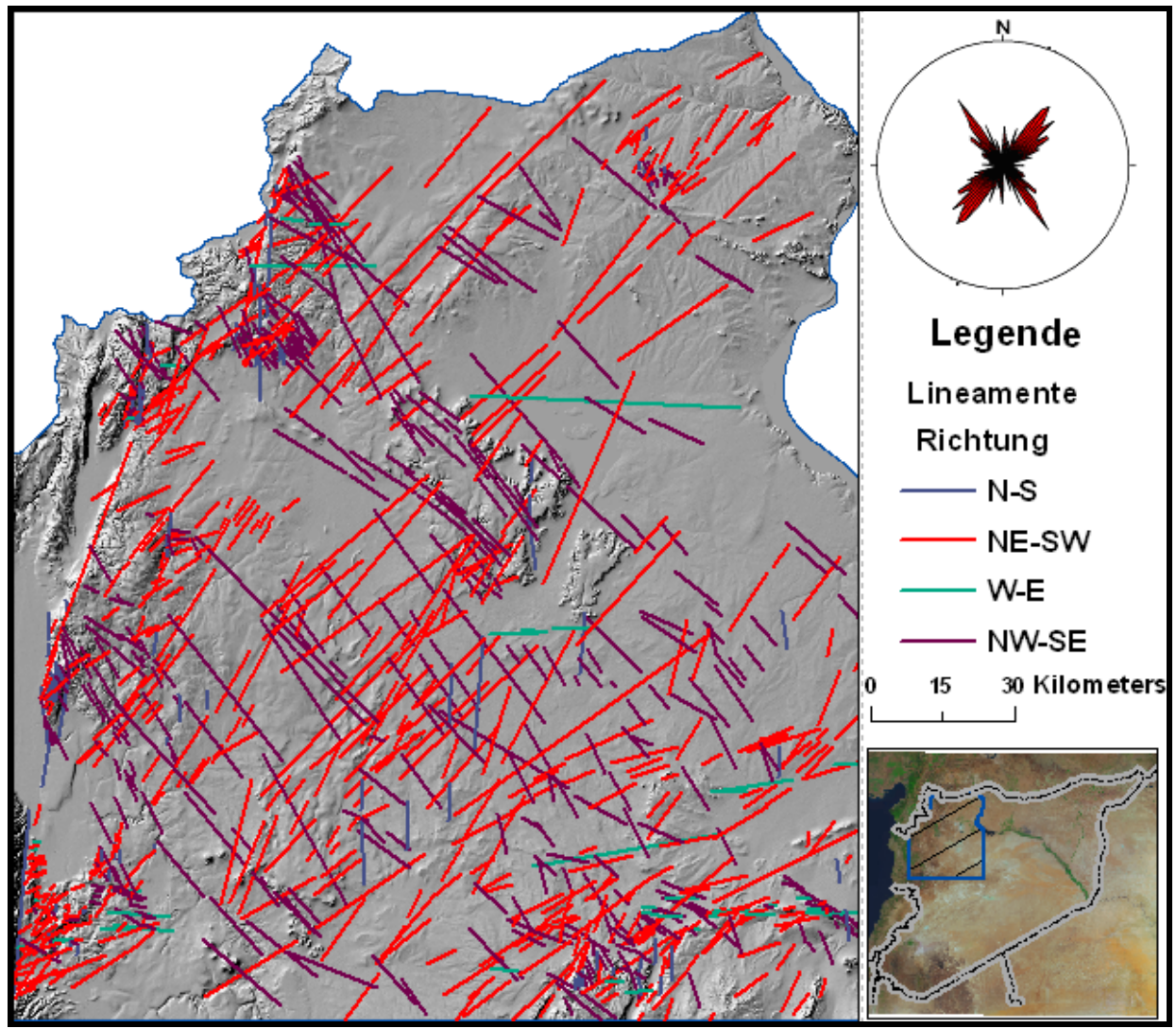

Abb. 6-9: Die Karte der ausgewerteten Lineamente kombiniert mit dem geschummerten Reliefmodel (Rosendarstellung oben rechts für alle Lineamente)

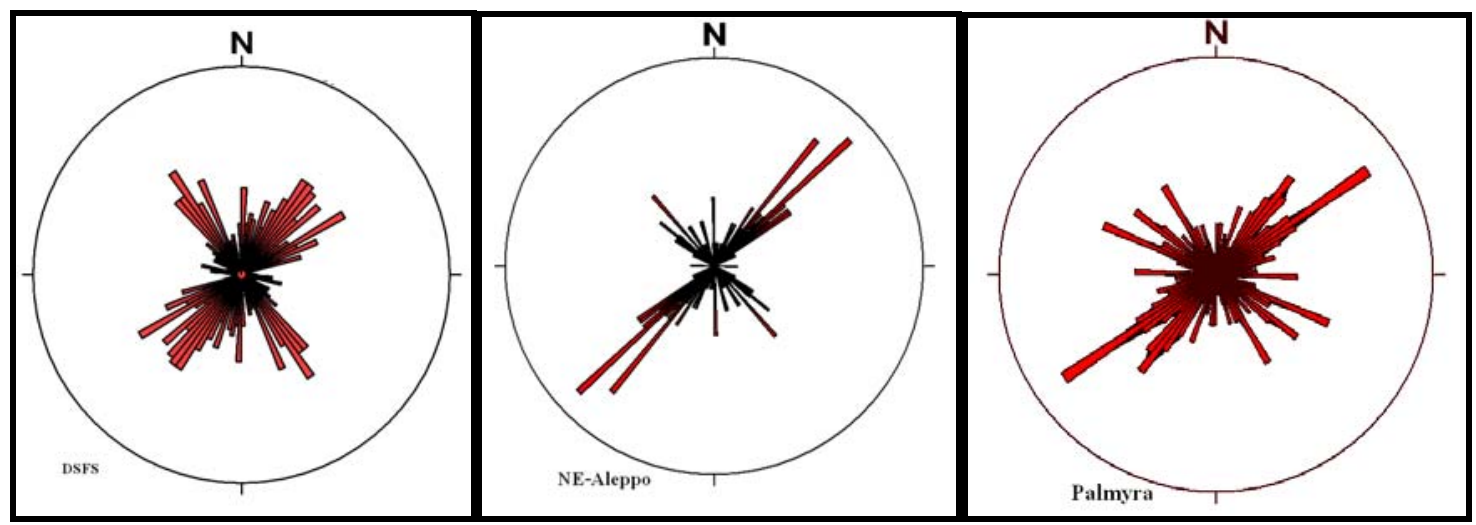

Abb. 6-10: Die Rosendarstellung der Lineamente aus Bereichen des DSFS, NEAleppo-Gebiets und der Palmyra-Ketten

Zum Vergleich der auskartierten Lineamente mit den Literaturdaten ist es zu Beginn wichtig, die alte Hypothese der rhegmatischen Scherzone zu erklären. Diese Hypothese schlägt vor, dass die Kruste der Erde durch ein regelmäßiges System von orthogonalen Verschiebungsbrüchen, die aus globalen Druckbedingungen in der Erdgeschichte resultieren, zerbrochen wird. Diese Hypothese wurde von WOLFART (1967) für die Erklärung der orographisch-morphologischen Gestaltung der Oberfläche im Nordwesten Syriens 
verwendet. Seine Annahme beruht darauf, dass durch Untersuchung des Entwässerungsnetzes rhegmatische Kluftscharen ermittelt werden können, deren Kreuzungswinkel häufig etwas unter $90^{\circ}$ liegt. Auf dieser Basis sei der Hauptteil des Gebiets zwischen dem Euphrat und Al-Ghab Graben im Bereich einer großen Zonale (Bekaa-Zonale), die südwestlich von Damaskus im spitzen Winkel von ca. $20^{\circ}$ auf die südlichen Palmyra-Ketten trifft und sich über $430 \mathrm{~km}$ nach Nordosten erstreckt. Er vermutete, dass die Intensität der Bekaa-Zonale nach NE abnimmt. Dieser zur Folge konnten nach seiner Meinung im Vergitterungsbereich mit der Aleppo-Hebung südlich von Aleppo seichte Senken entstehen (Al-Jabboul, El-Matah und Al-Kharaitch). Diese Senken seien an rhegmatische Zonen gebunden, die im peripheren Bereich der Senken am gerichteten Verlauf einzelner Talzüge zu erkennen sind (Abb. 6-10).

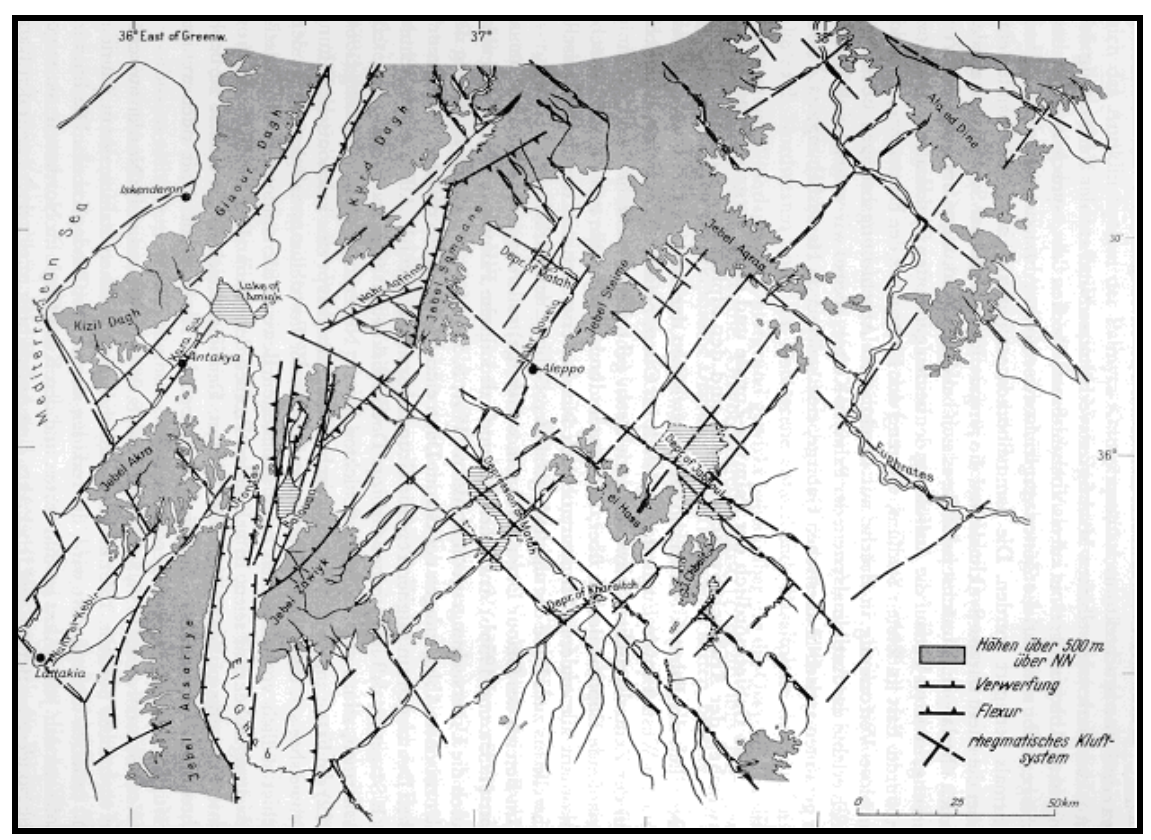

\section{Abb. 6-11: Rhegmatische Orientierung der orographischen Züge und des Entwässe- rungsnetzes (WOLFART, 1967)}

Zum Erklären der Verschiebungsdifferenz zwischen dem südlichen Teil des DSFS, der eine Verschiebung in Höhe von $105 \mathrm{~km}$ zeigt, und dem nördlichen Teil, der nur eine Verschiebung in Höhe von $25 \mathrm{~km}$ markiert, wurden mehrere Modelle vorgeschlagen. Der nördliche Teil liegt nördlich von der Abzweigung der Palmyra-Ketten. Diese Abzweigung befindet sich südwestlich von Damaskus. In Bezug darauf ist es noch eine Verschiebung in Höhe von ca. 80km innerhalb Syriens zu suchen. Ein Modell von WALLEY 
(1988) modifiziert von CHAIMOV et al. (1990) schlägt vor, dass eine Bewegung über $80 \mathrm{~km}$ in Richtung NE-SW entlang eines links-lateralen verzweigten Abschiebungssystems stattfand (Abb. 6-11).

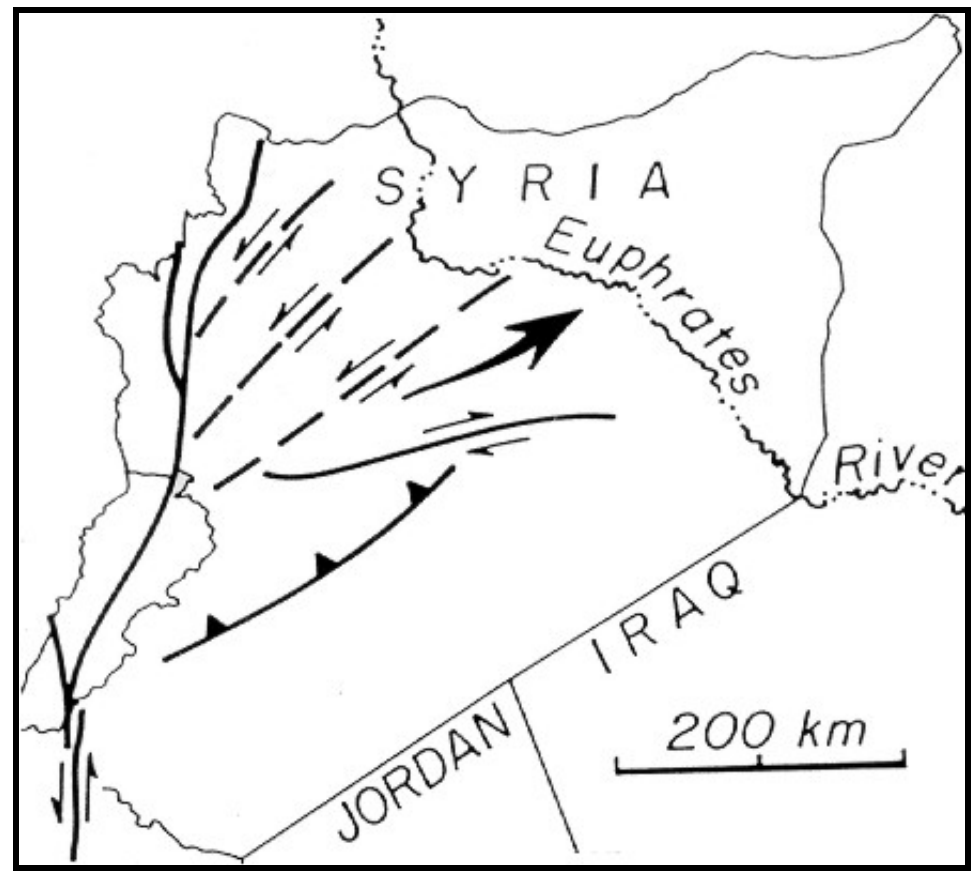

Abb. 6-12: Seitenverschiebungsmodell NW-Syrien (CHAIMOV et al., 1990)

In einer Studie über Paläospannung in dem Gebiet zwischen dem DSFS und dem Euphrat dokumentierten ZANCHI et al. (2002) in mehreren Teilen des Gebiets E-W Falten und NS Klüfte in den Kreide-, Paläogen- und Torton-Schichten, die von normalen dip-slipStörungen mit der Richtung NW-SE gekreuzt sind. In dem westlichen Bereich dokumentierten sie neben den N-S verlaufenden Toten-Meer-Verwerfungen in dem TortonKalkstein auch strike-slip-Störungen in Richtung NW-SE und NE-SW.

Auf diese Weise lässt der Vergleich zwischen den Ergebnissen dieser Arbeit und der Literatur eine ausdrückliche Übereinstimmung zwischen den beiden Daten feststellen. Diese drei Tendenzen der Lineamente stimmen mit der tektonischen Entwicklung des Gebiets überein. Die allergrößte Anzahl der Lineamente streicht $\mathrm{NE} \pm \mathrm{SW}$. Diese Orientierung entspricht dem Streichen der Hauptrichtung der Strukturen in den Palmyra-Ketten und Antilibanon bzw. Bekaa-Zonale. Ein anderer Vergleich zwischen der Karte der Lineamente und einer thematischen schematischen tektonischen Karte, die ober- und unterirdische Strukturen in dem Gebiet enthält, zeigt eine starke Verbindung zwischen den überdeckten tektonischen Strukturen und den morphologischen Eigenschaften auf. 


\title{
Ka p i t e l 7
}

\author{
Erstellung der
}

hydrogeomorphologischen Datenbank 


\section{Erstellung der hydrogeomorphologischen Daten- bank}

Die sorgfältige geomorphologische Kartierung eines Gebiets und seiner Elemente ist für vielfältige praktische Zwecke (die Planung, die Land- und Forstwirtschaft, die Bauprojektierung, die Erkundung bzw. die Erschließung der geologischen Ressourcen und die Kartographie) von größerer Bedeutung. Für bestimmte Zwecke ist es oft erforderlich, den Inhalt der geomorphologischen Karte durch weitere Informationen (Landnutzung, Böden, Lithologie etc.) zu ergänzen (DEMEK, 1976). Für die Untersuchung erneuerungsfähiger Naturressourcen, wie z. B. Grundwasser, stellt die räumliche geomorphologische Darstellung und Klassifikation ein hervorragendes Hilfsmittel zur Verfügung. Auf diese Weise wurde unter Einsatz der angewandten Geomorphologie die hydrologisch/ hydrogeologisch orientierte, geomorphologische Erkundung und Kartierung gut entwickelt (die Hydrogeomorphologie). Hierbei können die qualitativ und quantitativ erfassenden geomorphologischen Merkmale (wie Neigung, Exposition, Grundrisstyp der Reliefformen etc.) mit tektonisch-lithologischen Eigenschaften, Klima, Böden, Vegetation und Landnutzung integriert werden, um den Oberflächenabfluss, die Infiltration und die Grundwasserneubildung zu erforschen.

Für diesen Ansatz wurde der Bereich zwischen den Palmyra-Ketten und den abflusslosen Becken ausgewählt. Dieser Entscheidung liegen verschiedene Faktoren, vor allem die Mannigfaltigkeit der geomorphologischen Phänomene, die unmittelbare positive und negative Effekte auf das Wasserverhalten haben, zugrunde. Mit dem Ziel, eine Datenbank zu erstellen, die den Studien der Oberflächen- und Grundwasserressourcen und der damit verbundenen Problemen dienen kann, wurden die gesamten Datenmaterialien nach der Aufbereitung und Optimierung in einer Datenbank gespeichert (Tab. 7-1).

\section{Tab. 7-1: Inhalt der hydro-geomorphologischen Datenbank}

\begin{tabular}{|l|l|}
\hline Karten & $\begin{array}{l}\text { Topographie (Wadis) - Geologie (Lithologie, Stratigraphie, Tektonik } \\
\text { und Quartärablagerung) - Hydrogeologie (Grundwasserleiter)- Böden - } \\
\text { Landnutzung. }\end{array}$ \\
\hline Tabelle & Bohrungen und chemische Zusammensetzung des Grundwassers. \\
\hline Darstellung & $\begin{array}{l}\text { eingescannte topographische und geologische Karten, Fotos, geologi- } \\
\text { sche Querprofile, Schematische Darstellung. }\end{array}$ \\
\hline Bilder & Satellitenbilder (TM, ETM+, ASTER). \\
\hline
\end{tabular}


Als Beispiel für die Einsatzmöglichkeiten dieser hydrogeomorphologischen Datenbank wurde hier versucht, eine Aussage über das Grundwasserneubildungspotential zu ermitteln. Dies geschah durch die Kopplung verschiedener Themen mit Berücksichtigung ihrer Einflüsse auf Wasserinfiltration, -haltung und -verlust (Abb. 7-1).

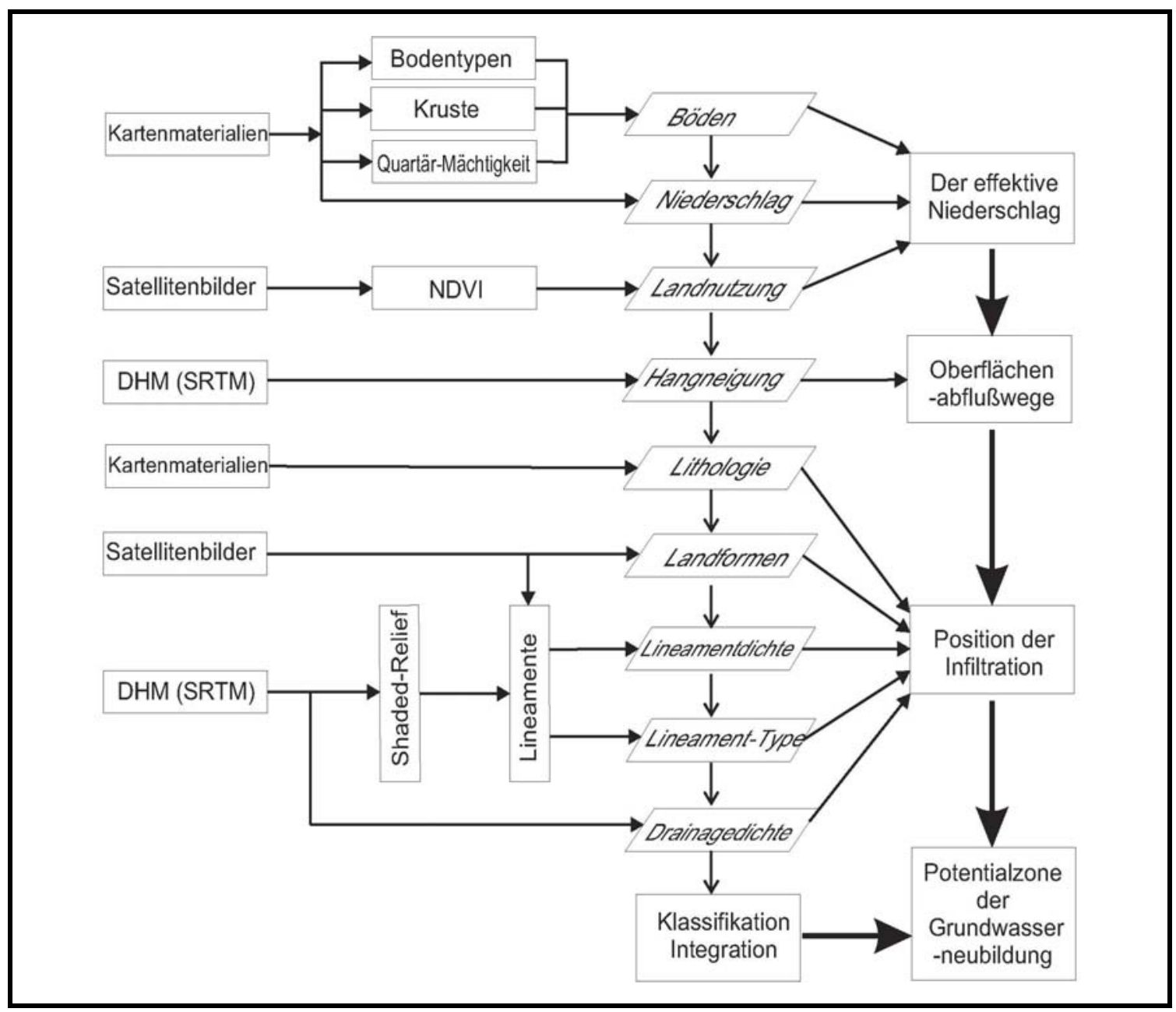

Abb. 7-1: Ablaufdiagramm der hydrogeomorphologischen Darstellung

Die thematischen Ebenen, die als Vektordaten (die Geomorphologie, Lineamenten und Entwässerungsnetz) gespeichert waren, wurden in Rasterdaten umgewandelt. Danach erhielten alle Kategorien jeder Ebene ihre Gewichte. Die unterschiedlichen Kategorien wurden entsprechend ihrem Wert in Bezug auf die anderen Kategorien in der gleichen thematischen Ebene gewichtet. Dadurch war die Kopplung auf der Basis der Rasterdaten möglich. 
Die folgenden Gewichte wurden gemäß der verfügbaren Informationen zum Teil probabilistisch und zum anderen Teil deterministisch gesetzt: sehr gut (1), gut (2), mäßig (3), schlecht (4), sehr schlecht (5), so basiert z. B. die Gewichtung der thematischen Niederschlagsebene auf der klimatischen Unterteilung des ganzen Landes in vier Bereiche. Ebenso wurde die Ebene der Hangneigung auf eine einfache Weise durch die Untergliederung der vorhandenen Werte gewichtet (Tab. 7-2).

Tab. 7-2: Sechs thematische Ebenen der Datenbank mit ihren Gewichten

\begin{tabular}{|c|c|c|c|c|c|}
\hline Kriterien & Klassen & Gewicht & Kriterien & Klassen & Gewicht \\
\hline Böden & \multicolumn{2}{|c|}{ Siehe Tabelle (7-3) } & \multirow{9}{*}{ 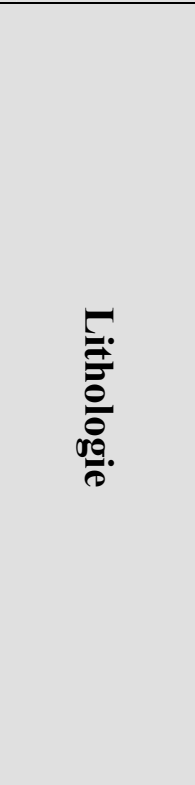 } & \multirow{2}{*}{$\begin{array}{l}\text { Alluvial } \\
\text { Proluvial }\end{array}$} & \multirow{2}{*}{1} \\
\hline \multirow{6}{*}{ 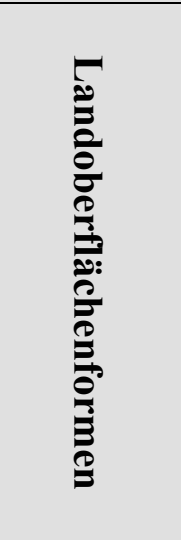 } & Wadibett & 1 & & & \\
\hline & arides Fluvial \& Glacis & 2 & & $\begin{array}{l}\text { massive Do- } \\
\text { lomite oder } \\
\text { Kalkstein }\end{array}$ & 2 \\
\hline & Basalt-Verwitterung & 3 & & $\begin{array}{l}\text { Konglomerate } \\
+ \text { Sandstein }\end{array}$ & 2 \\
\hline & Horst & 3 & & Basalt & 3 \\
\hline & altes Fluvial \& Pediment & 4 & & $\begin{array}{l}\text { dolomitischer } \\
\text { Tonkalkstein }\end{array}$ & 3 \\
\hline & Denudationsmerkmale & 4 & & $\begin{array}{l}\text { kreidiger } \\
\text { Tonkalkstein }\end{array}$ & 4 \\
\hline \multirow{5}{*}{ 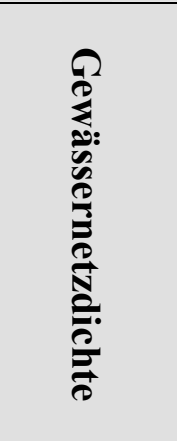 } & $0.00-0,39 \mathrm{~km}^{2}$ & 1 & & $\begin{array}{l}\text { laminierter } \\
\text { Kalkstein }\end{array}$ & 4 \\
\hline & $0.39-0,77 \mathrm{~km}^{2}$ & 2 & & $\begin{array}{ll}\text { Gips } & \text { oder } \\
\text { Mergel } & \end{array}$ & 5 \\
\hline & $0.77-1,16 \mathrm{~km}^{2}$ & 3 & \multirow{3}{*}{ 泾 } & $\begin{array}{l}\text { bewässerte } \\
\text { Felder }\end{array}$ & 3 \\
\hline & $1.16-1,54 \mathrm{~km}^{2}$ & 4 & & Regenfelder & 4 \\
\hline & $1.54-1,93 \mathrm{~km}^{2}$ & 5 & & $\begin{array}{l}\text { natürliche } \\
\text { Vegetation }\end{array}$ & 5 \\
\hline \multirow{5}{*}{ 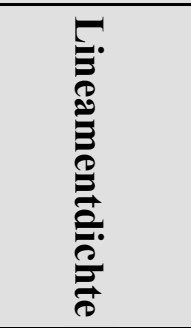 } & $0.00-0,31 \mathrm{~km}^{2}$ & 5 & \multirow{4}{*}{ 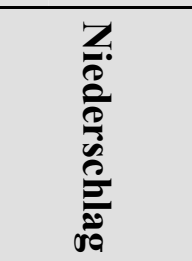 } & $150-200 \mathrm{~mm}$ & 4 \\
\hline & $0.31-0,62 \mathrm{~km}^{2}$ & 4 & & $200-250 \mathrm{~mm}$ & 3 \\
\hline & $0.62-0,93 \mathrm{~km}^{2}$ & 3 & & $250-300 \mathrm{~mm}$ & 2 \\
\hline & $0.93-1,24 \mathrm{~km}^{2}$ & 2 & & $300-400 \mathrm{~mm}$ & 1 \\
\hline & $1.24-1,55 \mathrm{~km}^{2}$ & 1 & \multirow{5}{*}{ 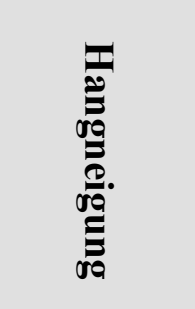 } & $0^{\circ}-5^{\circ}$ & 1 \\
\hline \multirow{4}{*}{ 突 } & \multirow{4}{*}{\multicolumn{2}{|c|}{ Siehe Tabelle (7-4) }} & & $5^{\circ}-10^{\circ}$ & 2 \\
\hline & & & & $10^{\circ}-20^{\circ}$ & 3 \\
\hline & & & & $20^{\circ}-30^{\circ}$ & 4 \\
\hline & & & & $30^{\circ}-40^{\circ}$ & 5 \\
\hline
\end{tabular}




\subsection{Ermittlung und Gewichtung der thematischen Ebenen}

Die nächsten Schritte erörtern die Kriterien, die bei der Gewichtung der verschiedenen Ebenen verwendet wurden. Die Abbildung (7-4) zeigt die klassifizierten thematischen Karten, die ermittelt und anschließend integriert sind, um die Karte des Grundwasserneubildungspotentials zu ermitteln.

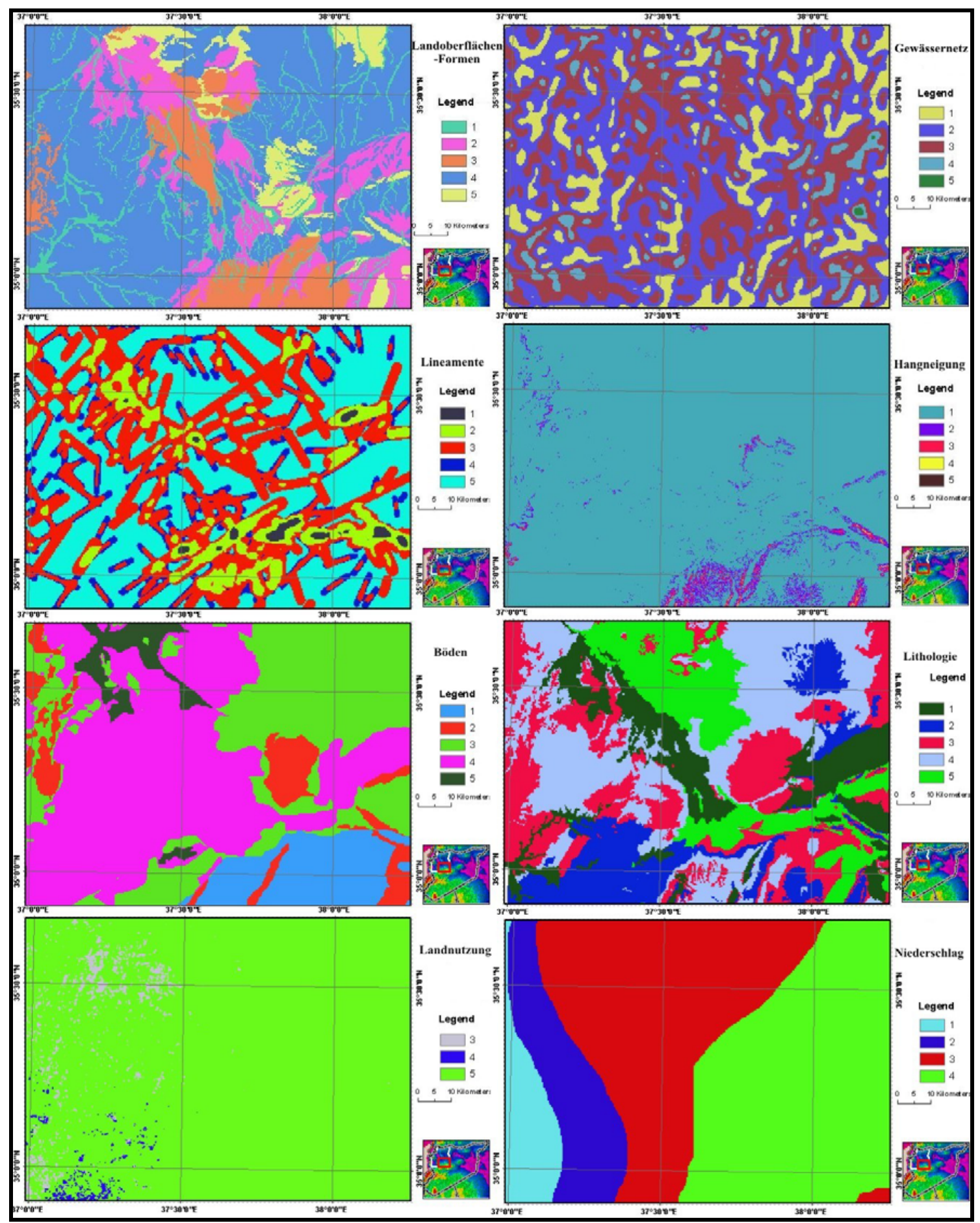

Abb. 7-2: Thematische Karten der gewichteten Kategorien 
Bei der Gewichtung der Böden-, Landnutzung- und Lithologieebene wurden teilweise die Parameter, die in der Karte der Hydrogeologie und Zonensanierung (SELKHOZPROMEXPORT, 1982) festgelegt wurden, verwendet. Bei dieser Karte handelt es sich um die hydrographischen Becken von Aleppo, Orontes und Damaskus. Bei der Erstellung dieser Karte wurden die Becken in morphogenetische Einheiten unterteilt, und verschiedene Parameter (wie Durchlässigkeit, Grundwasserspiegelerhöhung nach der Bewässerung, und Mächtigkeit der Deckschichten) definiert. Diese Arbeit enthält allerdings keine direkten Informationen über das Grundwasserneubildungspotential. Im Hinblick auf das hier betrachtete Gebiet hat diese Arbeit nicht das ganze Gebiet abgedeckt (Abb. 7-3). Deswegen wurden die nicht abgedeckten Teilen durch den Vergleich mit anderen charakteristisch ähnlichen Teilen parametrisiert.

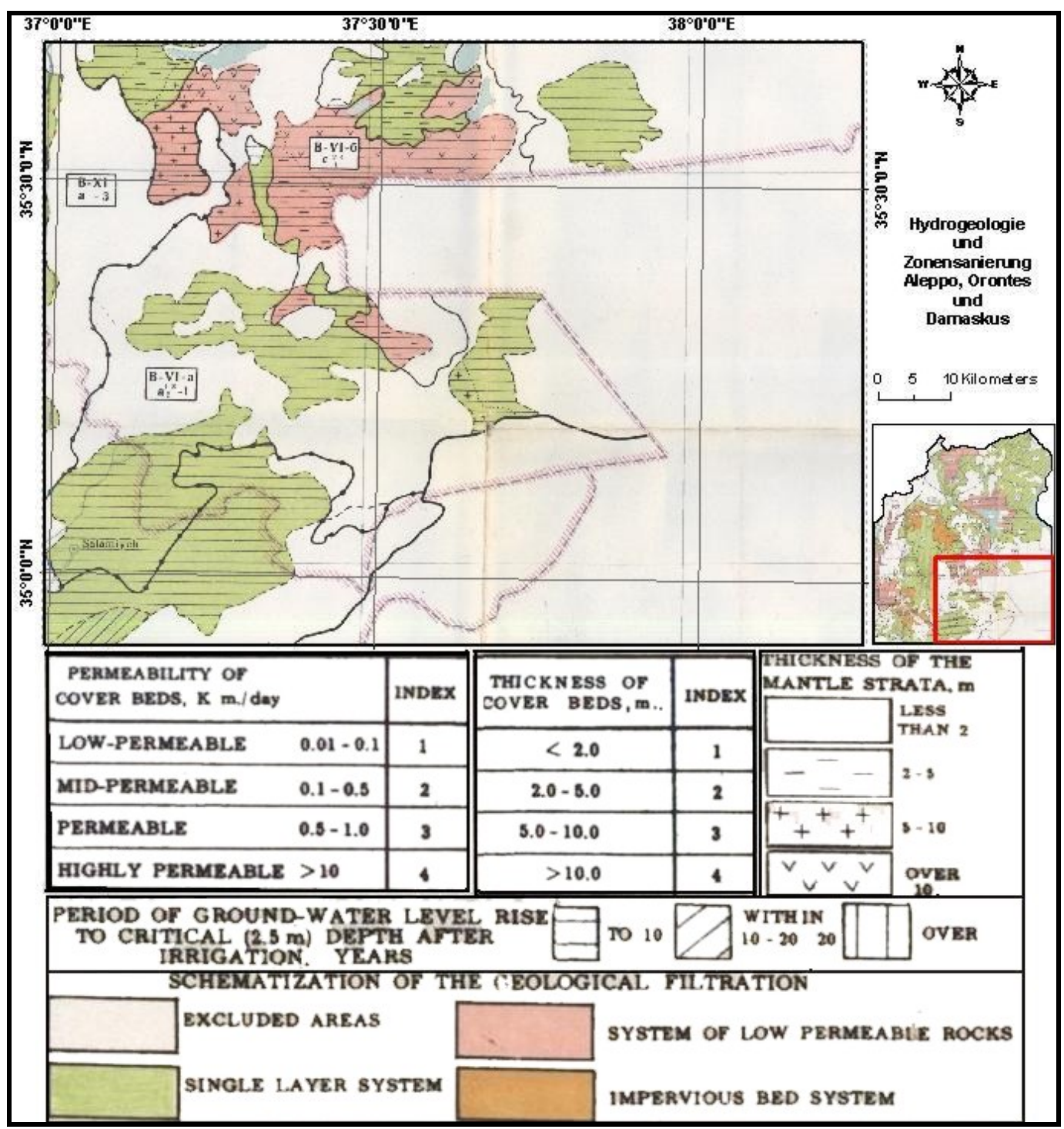

Abb. 7-3: Ausschnitt der Hydrogeologiekarte mit den Parametern 


\subsubsection{Thematische Ebene der Böden}

Die Wasserhaltungskapazität eines Gebiets ist von dem Bodencharakter und seiner Permeabilität stets abhängig. Bei der Ermittlung der thematischen Ebene der Böden wurden die Bödentypen, Mächtigkeit der Böden- bzw. quartärer Ablagerung und die Krusten berücksichtigt.

Durch die Kombinierung der Satellitenbilder mit der Bodenkarte Syriens wurden die Bödentypen visuell gezeichnet und abgegrenzt. Die vorhandenen Böden sind Aridisols (Calciorthids und Gypsiorthids), Paleorthids, Torriorthents und Inceptisols. Der größte Teil des Bereichs wird von Aridisols (Yellow Soils) bedeckt, wobei Calciorthids und Gypsiorthids bei weiten vorherrschenden Bodentypen sind. Im nordöstlichen Teil begünstigt sowohl die geomorphologische Lage als auch das mit Salzen belastetes $(>5 \mathrm{~g} / \mathrm{l})$ Grundwasser die Gips- und Salzanreicherungen in dem Al-Kharaitch Sabkha. Dabei handelt es sich in der Regel chloridische und sulfatische Na-, Ca- und Mg-Salze. Das Calciorthids ist über den vorwiegenden merglig-kreidigen Schichten des Paläogens verbreitet, und weist häufig an der Oberfläche oder in geringerer Tiefe harte Kalkkrusten auf. Die sich über Krusten befindenden Böden sind sehr steinig und kieselig. Die Verhältnisse hier sind günstig für den Ackerbau, daher wurden die Kalkkrusten entfernt und Bäume, wie z. B. Olivenbäume, gepflanzt. Weiter entfernt im Osten bildeten sich kompakte Kalkkrusten über pliozänen und quartären Schottern. In solchen Teilen sind sie nur von einem dünnen Schleier Feinmaterials überdeckt (Abb. 7-4).

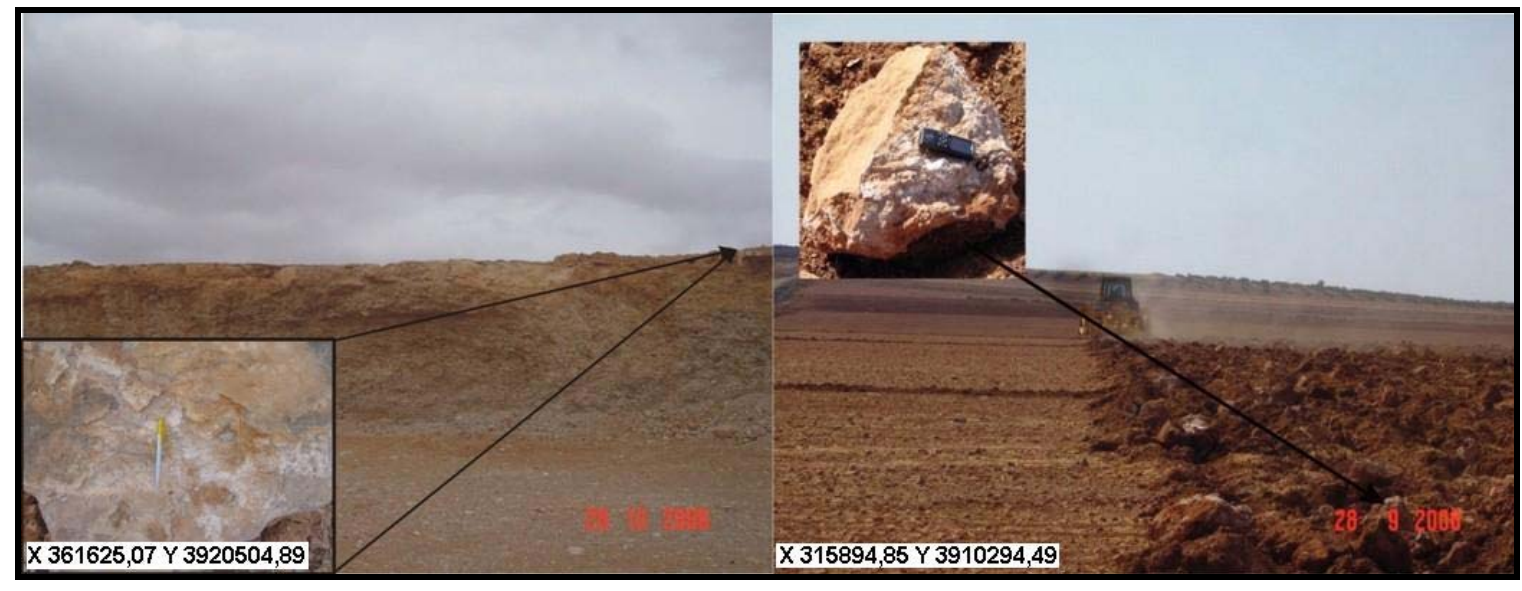

Abb. 7-4: Von Kalkkrusten überdeckte fluviale Ablagerung (links) Kalkkrustenbeseitigung (rechts) 
Neben den Aridisols nehmen die Inceptisols den flächenmäßig größten Raum ein. In ihrem Profil bzw. auf der Oberfläche sind häufig Stein- und Kiespflaster zu finden. Sie haben auch oft unter der Oberfläche einen Kalkhorizont. Trotzdem sind sie im Allgemeinen produktive Böden. Auf den Palmyra-Ketten erscheinen die Paleorthids. Dies sind Böden mit einem oberflächennah anstehenden petrocalcic Horizont, der sich aus dem Kalkanreicherungshorizont eines früheren Bodens entwickelt hat. Bei der Ermittlung der thematischen Ebene der Böden wurde beachtet, dass die große Mächtigkeit der Böden und der Quartärablagerung negative Effekte auf die Grundwasserneubildung hat, weil das Wasser in dem Bodenprofil lange verweilen wird und von der Vegetation verwendet werden kann oder verdunstet (Abb. 7-5). In den Bereichen, wo das Bodenprofil nicht mächtig $(30-70 \mathrm{~cm})$ ist, kann das Wasser den Kalkstein erreichen und durch Klüfte infiltrieren. Diese Tatsache wurde auch in dem Basaltgebiet berücksichtigt, weil das Bodenprofil dort sehr flach ist.

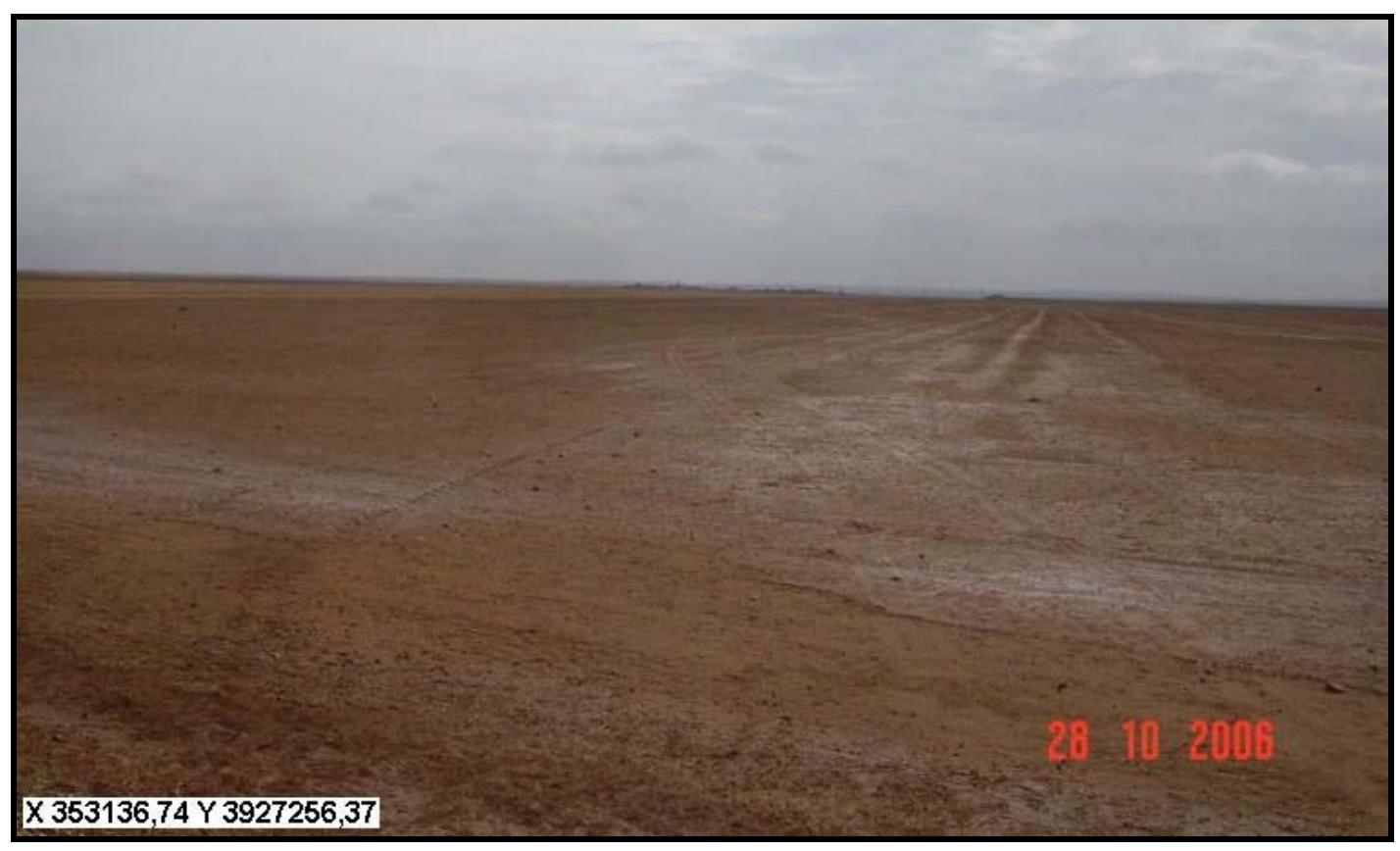

Abb. 7-5 Böden im Zentrum des Gebiets zwei nach Regenereignis

Tab. 7-3: Gewichtung der verwendeten Daten für Ermittlung der Bodenebene

\begin{tabular}{|c|c|c|c|c|c|}
\hline Ebene & Kategorie & Gewicht & Ebene & Kategorie & Gewicht \\
\hline \multirow{5}{*}{ 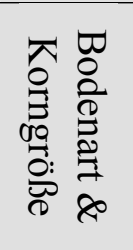 } & Grobkorn-Paleorthids & 3 & \multirow{5}{*}{ 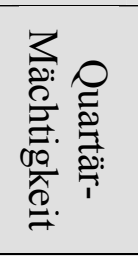 } & $0 \mathrm{~m}$ & 1 \\
\hline & Mittelgrob-Paleorthids & 4 & & $0-0,5 \mathrm{~m}$ & 2 \\
\hline & Grobkorn-Aridisols & 2 & & $0,5-2 \mathrm{~m}$ & 3 \\
\hline & Mittelgrob-Inceptisols & 1 & & $2-5 m$ & 4 \\
\hline & Feingrob-Inceptisols & 2 & & $>5 \mathrm{~m}$ & 5 \\
\hline Kruste & Kahlkruste & 4 & Kruste & ohne & 2 \\
\hline
\end{tabular}




\subsubsection{Thematische Ebene der Landoberflächenformen}

Die Geomorphologie hier hängt von den geologischen Strukturen und dem regionalen geologischen $\mathrm{Bau}$ ab und ist im Allgemeinen ein Resultat von Deformationen und den darauf folgenden Denudationen. Sie ist im Hinblick auf die Hydrogeomorphologie in drei Hauptformen zu unterteilen: die Wasserscheide, die Wasserkollektoren und das abflusslose Becken (Sabkha Al-Kharaitch). Die Wasserscheidebereiche werden durch die Hochländer dargestellt und in zwei Hauptgliederungen unterteilt: die Palmyra-Ketten und die Basalthochebene. Die Wasserkollektoren werden durch die topographischen Senken und die Entwässerungslinien gebildet. Die dritte hydrogeomorphologische Einheit ist die Ebene des abflusslosen Beckens, das hauptsächlich aus lakustrinen Ablagerungen besteht.

Für diesen Bereich wurden neben der Landsatdaten auch ASTER-Daten ausgewertet, um die geomorphologische Darstellung der ausgewählten Region detaillierter zu ermitteln (Siehe Anhang 4). Außerdem wurde dieser Schritt durch eine gründliche Feldarbeit mit der Verwendung von GPS Messungen (GPS 12 von GARMIN) unterstützt, um die Beschreibung bzw. die Analyse der Geomorphologie in Verbindung mit der Realität durchzuführen.

Einige geomorphologische Einheiten wie Flutebenen, Ablagerungen in Senken und verschüttete Materialien der Wasserläufe sind typischerweise gute Quellen für Grundwasserneubildung, während strukturelle Erhebungen und Pedimente schlechte Zonen für Grundwasserneubildung sind. In diesem Sinne ist in dem genannten Bereich geomorphogenetisch zwischen fluviatilen Einheiten (Erosions- und Akkumulations-Einheiten) und strukturellen Einheiten zu unterscheiden.

Die Erosionseinheiten sind entweder lineare Formen (Wadis) oder flächenhafte Formen. Die Erosionsflächenformen sind die Pedimente (Fußflächen) oder Glacis. Bei dieser Definition wurde die eindeutige Unterscheidung zwischen den Felsflächen als Pedimente und den lockeren Verwitterungsmaterialien als Glacis verwendet (BESLER, 1992). Die Glacis bilden schotterbedeckte, flach geneigte Ebenen am Rand der Palmyra-Ketten. Sinngemäß werden die kleinen Schutthalden oder Schuttflächen, die am Ausgang der kleinen Wadis am Rand der topographischen Höhen sind, als Glacis bezeichnet bzw. kartiert. Die Glacis sind gelegentlich von Wadis (Fluviatile Erosion) eingeschnitten, wobei sie in einigen Teilen wie Glacisterrassen aussehen (Abb. 7-6). 
Die Unterscheidung zwischen Glacis und Schwemmfächer basiert auf folgendem:

(i) auf Glacis ist die Abtragung größer als die Akkumulation, wodurch sich nur ein dünner Sedimentschleier ausbilden kann, und

(ii) auf Schwemmfächerflächen ist dagegen die Akkumulation größer als die Erosion.

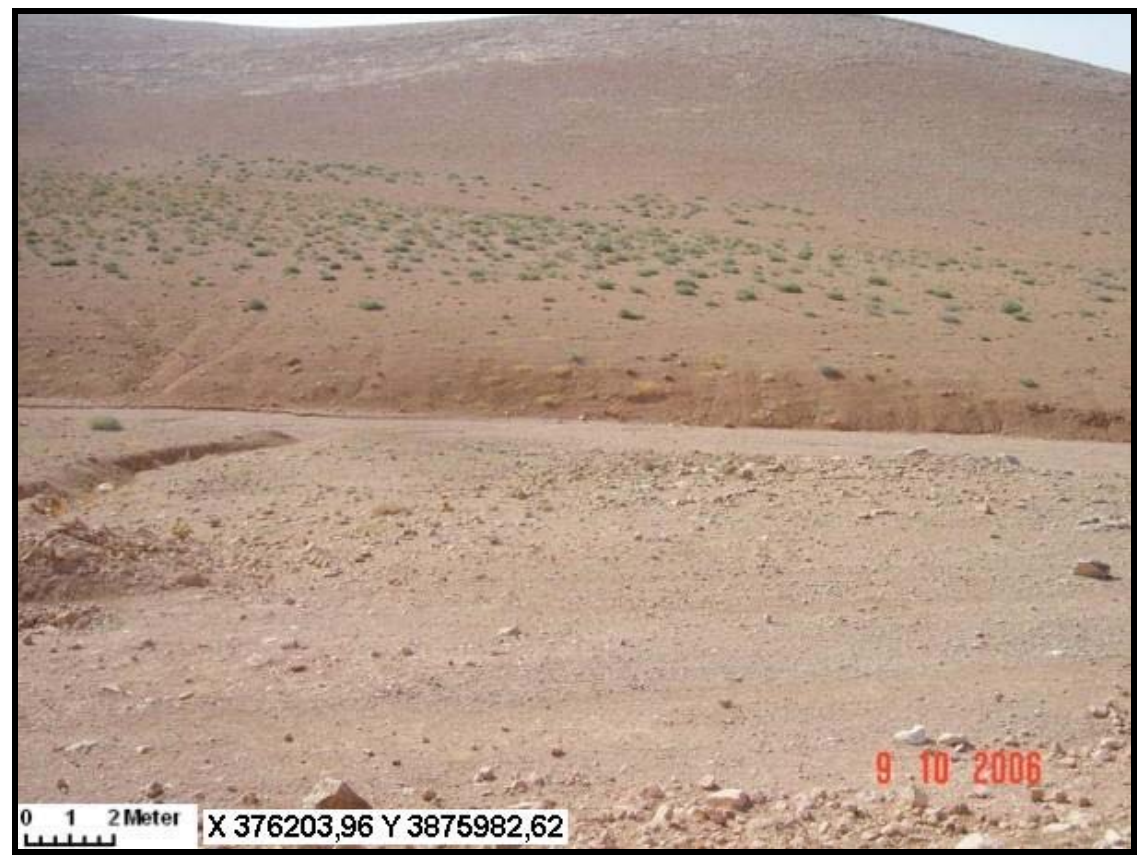

Abb. 7-6: Glacisterrassen am Rand der Palmyra-Ketten (Blickrichtung nach SE)

Weiter entfernt von den Palmyra-Ketten gehen sowohl die Glacis als auch die Pedimente in breite Wadis und Schwemmfächer bzw. in das innere Becken von Al-Kharaitch über. Im Gegensatz zu den Pedimenten, die als schlechte Zone für die Wasserinfiltration einzustufen sind, stellen sich die Glacis hier als gute Zone dar. Der Grund ist, dass die letzteren das Regenwasser, insbesondere bei langsamen Regenereignissen, aufnehmen können und auf Grund ihrer Materialienzusammensetzung eine vertikale Infiltrierung erlauben.

Die Wadis in diesem Bereich, wie die anderen Wadis Syriens, sind im Altpleistozän (VAN LIERE, 1960/61) angelegt und zwar meistens entlang von linearen Strukturen (WOLFART, 1967). Dies hat sich bei der Auswertung von Lineamenten in Kapitel (6.5.2.2) bestätigen lassen. Mehrere Wadis sind in die alten Alluviallablagerungen eingeschnitten. Topographisch liegen sie ein paar Meter unter den umgebenden Ebenen. Einige dieser Wadis sind sehr klein und haben flache Seiten, wobei sie aus einem Abstand von mehr als einigen hundert Metern nicht erkennbar sind (Abb. 7-7). Andere Wadis sind kofferartig in ihrer 
Form und zeigen leichte Steigungen zu den vertikalen seitlichen Wänden. Die Länge der Wadis kann mehr als 10 Kilometer sein, doch die meisten sind kürzer als 5 Kilometer. Die Neigung der Oberfläche im Vorland der Palmyra-Ketten ist so schwach, dass ein schneller Oberflächenabfluss nicht anzunehmen ist. Im Bezug auf diese Eigenschaften ist das Infiltrationspotential von Oberflächenwasser entlang dieser Wadis sehr hoch (Tab. 72). Fast alle Wadis haben ein breites Sortiment an erodierten Materialien wie Lehm, schlecht sortierte Sedimente wie Sand und Kies. Diese Ablagerungen bilden schmale Auen $(20 \mathrm{~m}$ bis $50 \mathrm{~m})$, die durch eine oder mehrere verhältnismäßig flache Rinnen zergliedert werden kann. Die Auen können mitunter über 100m breit sein.

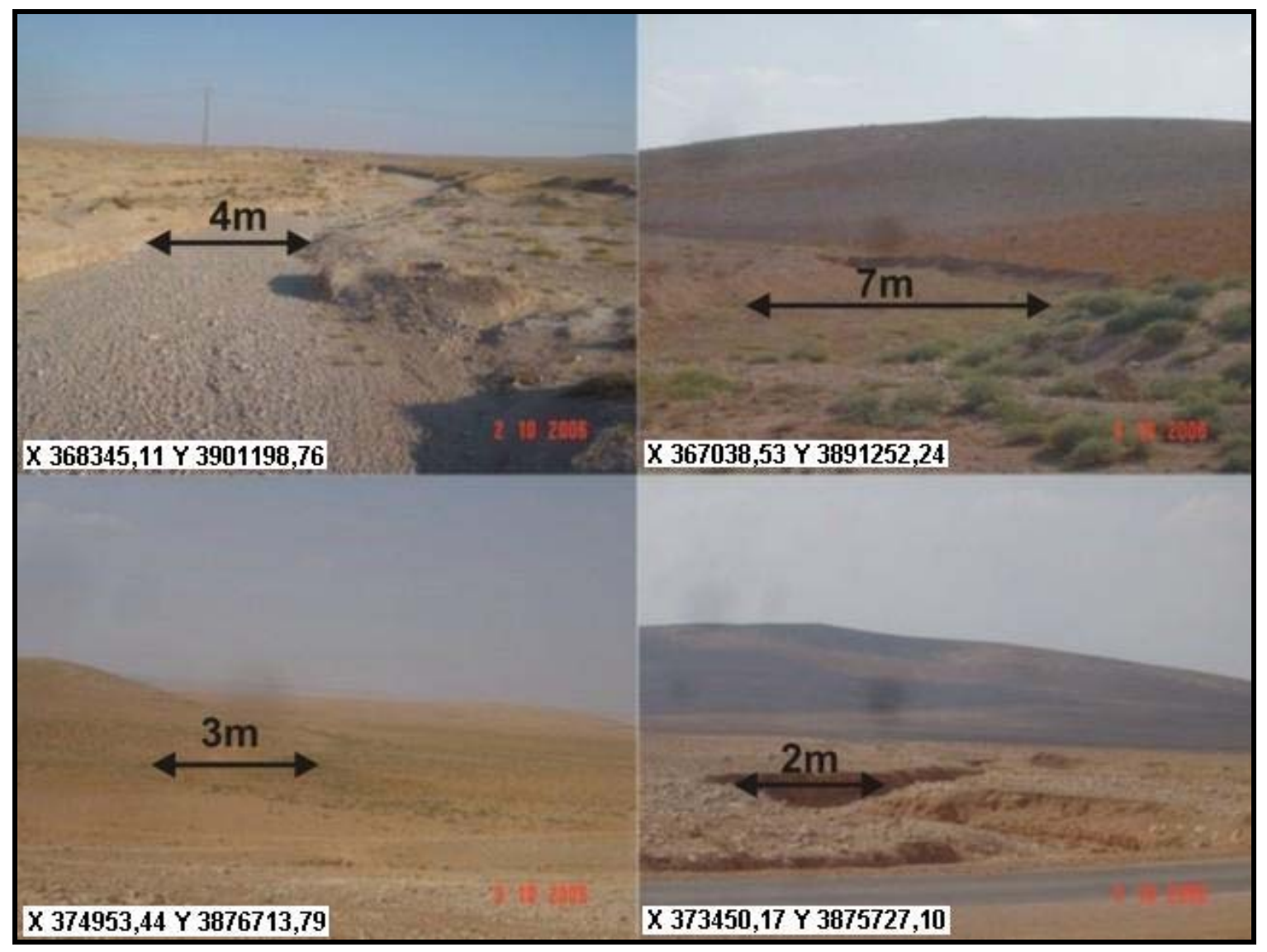

Abb. 7-7: Beispiele für verschiedene Wadis aus dem betrachteten Gebiet

Die rezenten Akkumulationsformen befinden sich einerseits an den Steilhängen am Rand der Palmyra-Ketten und des Basalt-Plateaus und andererseits in den großen Wadibetten, insbesondere in deren Unterlauf. An dem Basalt-Plateau bestehen die Ablagerungen in den Wadis aus den Verwitterungsprodukten, die zum größten Teil wahrscheinlich unter feuchten Bedingungen gebildet wurden. Andere Akkumulationseinheiten wie Aufschüt- 
tungsfächer sind in diesem Bereich zusätzlich vorhanden, die zum Teil fossile, fluviale, verfestigte, konglomeratische Decken aufweisen. Diese Formen verhindern teilweise die Wasserinfiltration. Doch in einigen Teilen können sie durch die Zerschneidung der Wadis geomorphologisch zu den Rumpfflächen (Denudationsflächen) gezählt werden. Auf Grund dessen wurden die Ablagerungen der Wadibetten, aride fluviale Akkumulationen und alte fluviale Ablagerungen, als sehr gute Zonen für das Infiltrationspotential eingestuft (Tab. 7-2). Der innere Teil des hier betrachteten Bereichs ist von den lakustrinen Ablagerungen eingenommen (Al-Kharaitch Sabkha). Im westlichen Teil stellen die Basaltlandoberflächen eine flache, schwach zertalte Tafel dar. Mit einer Mächtigkeit bis zu zehn Meter ragen sie mit dem vorgelagerten Mergel bis zu 300m über ihrem Umland auf. Die Wasserinfiltration hier ist angesichts der starken Verwitterung und der Zerklüftung als gemäßigt bis gut einzustufen. Die strukturell gebundenen oder bedingten Oberflächenphänomene befinden sich in dem südöstlichen Teil der Region in Form von Hügeln oder Horsten. Die Horste sind an den verfalteten Kalksteintafeln gebunden. Mehrere mäBig angehobene Flächen im Vorland der Palmyra-Ketten lassen sich als Pediplain (Denudationsformen) mit sehr flachgründigen Verwitterungsprodukten und Reste proluvialer Ablagerung kennzeichnen.

\subsubsection{Thematische Ebene der Gewässernetzdichte}

Das Gewässernetz ist als Resultat der Wechselwirkungen zwischen den klimatischen, geologischen, hydrologischen und biologischen Faktoren darzustellen. Die Gewässernetzdichte zeigt die Balance zwischen der Erosion und der Festigkeit der Landoberfläche, wobei das Klima und die Lithologie eine wichtige Rolle spielen. Andererseits ist der Gewässernetztyp stark an die geologischen Strukturen gebunden, insbesondere an Störungen bzw. Klüften. Deswegen steht sie im engen Zusammenhang mit dem Auftreten und der Verteilung des Grundwassers. Eine niedrige Gewässernetzdichte deutet auf eine hohe Porosität bzw. mögliches Wasserinfiltrationspotential. Dieses begründet sich aus der Tatsache, dass die Drainagedichte ein empfindlicher Parameter ist. Dieser Parameter liefert in vielen Fällen eine Verbindung zwischen den Attributen des Einzugsgebiets und den möglicherweise stattfindenden Prozessen, die entlang der Flussläufe auftreten können. Die thematische Karte der Entwässerungsdichte wurde vorbereitet, um die Region in Bereiche mit Grundwasserneubildungspotentialen zu kategorisieren (Abb. 7-2). 
Die Berechnung der Dichte wurde auf Basis der Kern-Dichte durchgeführt. In dieser Funktion der Kern-Dichte wurde über jeder Linie eine glatt gebogene Oberfläche zusammengefasst. In einem einstellbaren Suchradiusabstand vermindern sich die Werte mit der Entfernung von der betrachteten Linie. Die Werte erreichen Null in einem Abstand gleich dem Radiuswert. Die Dichte an jeder Ausgangsrasterzelle wird durch das Addieren der Werte aller Kernoberflächen, die den Rasterzellenmittelpunkt umrunden, ermittelt. Diese Kernberechnung für Liniendichten ist von der quadratischen Kernfunktion für Punktdichten nach SILVERMAN (1986) adaptiert.

\subsubsection{Thematische Ebene der Lineamente}

Die Lineamente in dem Gebiet sind von großer Bedeutung für die Erstellung der hydrogeomorphologischen Datenbank bzw. der Untersuchung der Grundwasserneubildung. Ihre Rolle wurde durch Anfertigung von zwei thematischen Ebenen dargelegt. Die Lineamente wurden in drei Typen gegliedert, weil nicht alle Lineamente die gleiche hydrogeologische Auswirkung haben:

1. Typ A: Lineamente stimmen mit den Verwerfungen der geologischen Karte überein,

2. Typ B: Lineamente stimmen mit den tektonischen Settings überein und

3. Typ C: vermutete strukturelle Lineamente.

Für jede Klasse wurden Pufferzonen mit der Funktion der Mehrfachpuffer (Multiple Ring Buffer) in den Abständen 500m, 1500m und 2500m von den Lineamenten gebildet. In der Pufferfunktion generiert ArcGIS Pufferpolygone in einem spezifizierten Abstand oder mehrere Abstände um den betrachteten Feature. Eine Überlappung kann durch die Auflösungsfunktion beseitigt werden (Abb. 7-8). Mit Berücksichtigung des Abstands und des Typs wurden die Pufferpolygone eingestuft (Tab. 7-4). Dieses Prinzip wurde ohne Unterscheidung zwischen den Lineamenttypen von HANSMANN et al. (1992) in Verbindung mit der Brunnenergiebigkeit erfolgreich angewendet.

In einem anderen Layer wurden Lineamente nach der Häufung (Dichte) klassifiziert. Die Berechnung der Dichte erfolgte auch wie die Gewässernetzdichte auf der Basis der Kernberechnung für Liniendichte. Die Häufung von Lineamenten ist immer mit der Gewässernetzdichte zu kombinieren. Entscheidend ist hier, dass eine geringe Gewässernetzdichte und hohe Häufung von Lineamenten dort auftreten, wo eine höhere örtliche Durchlässigkeit vorgegeben ist. Demzufolge wird die Wasserbewegung (Infiltration, 
Strömung) erleichtert. Die beiden thematischen Ebenen wurden kombiniert und erneut klassifiziert, um eine einzige Ebene für die Lineamente zu ermitteln (Abb. 7-2).

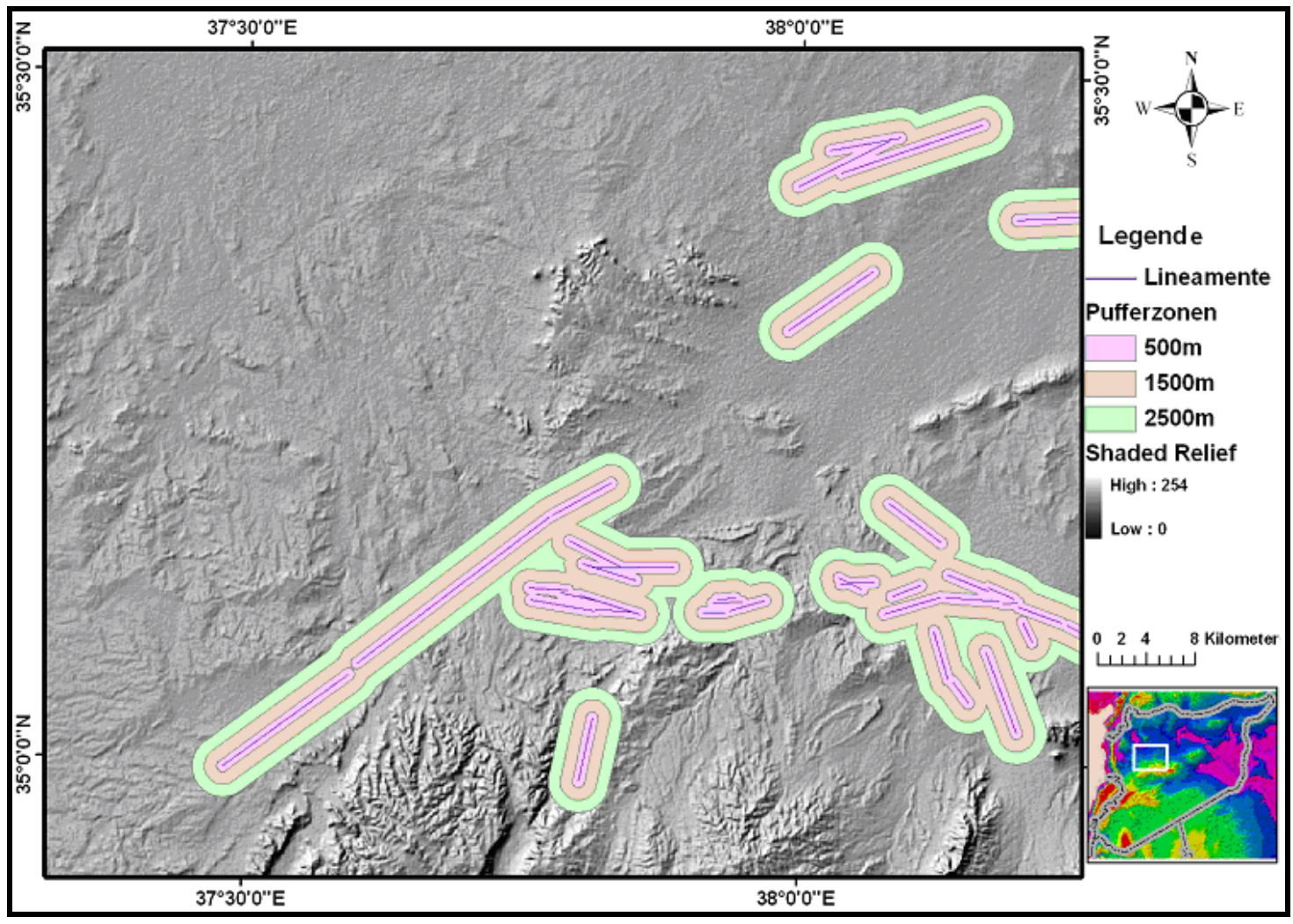

Abb. 7-8: Erschaffung mehrerer Pufferzonen über Lineamenten von Typ A

Tab. 7-4: Gewichtung der Lineament-Pufferzonen

\begin{tabular}{|c|c|c|c|c|c|}
\hline Art & $\begin{array}{c}\text { Abstand } \\
(\mathbf{m})\end{array}$ & Gewicht & Art & $\begin{array}{c}\text { Abstand } \\
(\mathbf{m})\end{array}$ & Gewicht \\
\hline A & 500 & 1 & B & 2500 & 4 \\
\hline A & 1500 & 2 & C & 500 & 3 \\
\hline A & 2500 & 3 & C & 1500 & 4 \\
\hline B & 500 & 2 & C & 2500 & 5 \\
\hline B & 1500 & 3 & \multicolumn{2}{|c|}{ Ohne Daten } & 0 \\
\hline
\end{tabular}

\subsubsection{Thematische Ebene der Lithologie}

Neben dem weit verbreiteten Basalt und merglig-kreidigen Kalkstein des Paläogen sind hier auch Kalksteine der Kreide und des oberen Miozän (Helvet) sowie Konglomerate des Neogen aufgeschlossen. Die hydraulische Leitfähigkeit des Basalts, sowohl die Horizontale als auch die Vertikale, ist mäßig bis gut, weil der Basalt stark verwittert und ein altes Relief (mehrere Wadis) bedeckt. Doch in den Teilen, wo die Kalkkrusten vorhanden sind, wurde die Wasserversickerung verhindert (Abb. 7-10). 
Die karbonathaltigen Gesteine zeigen außer dem Karbonatgehaltsunterschied mannigfache zusätzliche Eigenschaften wie Verkarstung, Klüftung und Mächtigkeit. Durch diese Beobachtungen und mittels der Klassifikation von Brahana et al. (1988) wurden die Gewichte der Lithologie gekennzeichnet (Abb. 7-10).

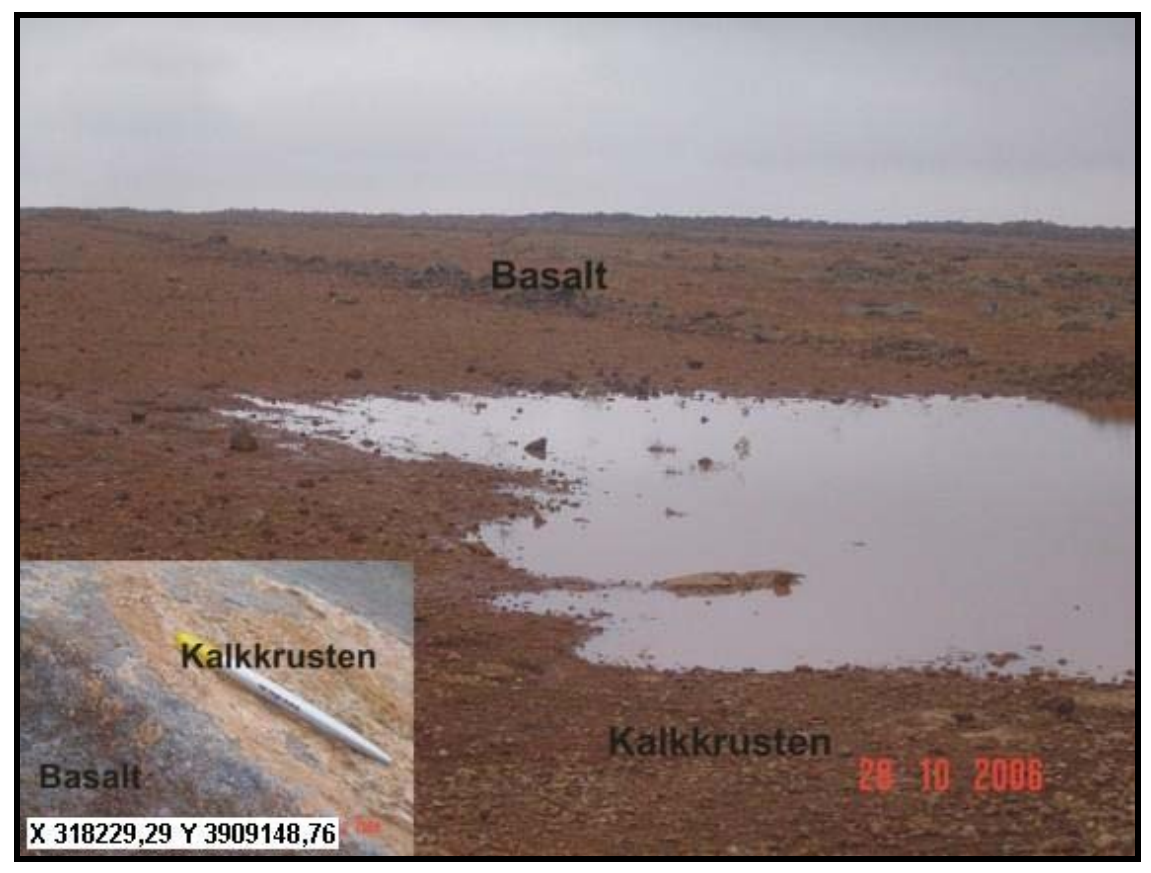

Abb. 7-9: Zwei Tage nach dem Regenereignis bleibt das Wasser auf dem Basalt stehen

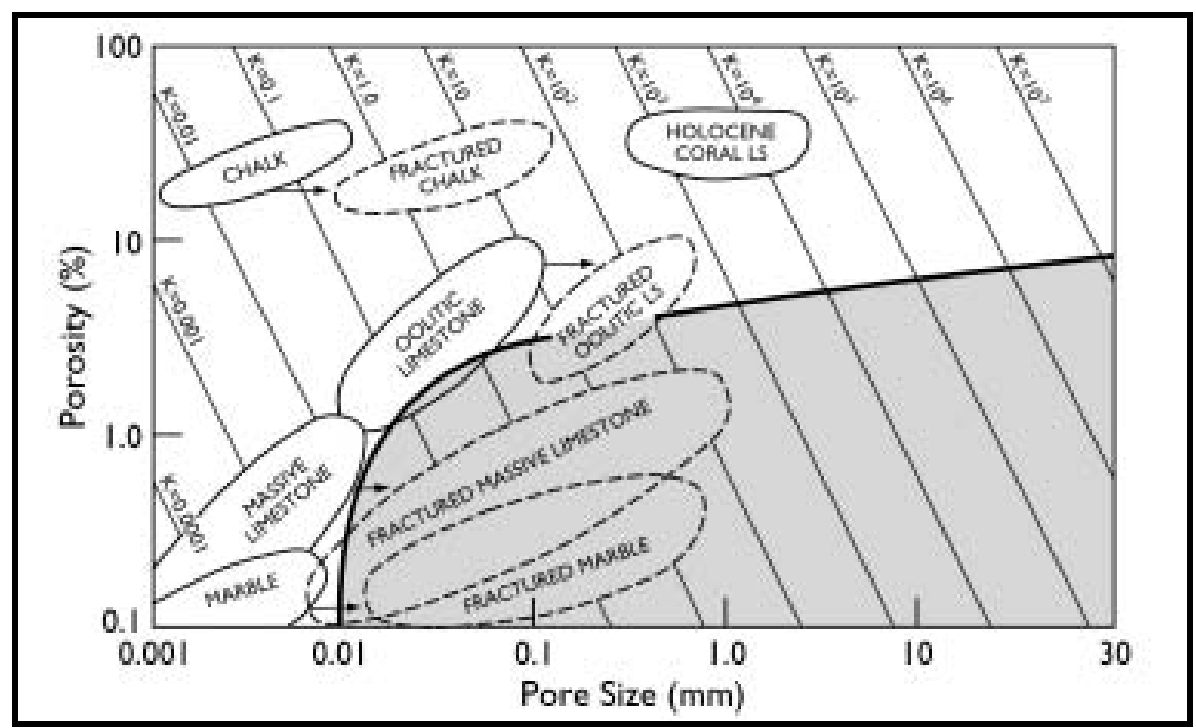

Abb. 7-10: Porosität, Porengröße und theoretische hydraulische Leitfähigkeit (m/Tag) mehrerer Karbonatgesteine (BRAHANA et al., 1988) 


\subsubsection{Thematische Ebene der Landnutzung}

Auf Grund ihres Einflusses auf den Wasserabfluss, die Evaporation bzw. die Grundwassergewinnung ist die Untersuchung von Landnutzung und Landbedeckung in der Grundwasserforschung unerlässlich. Das betrachtete Gebiet liegt in der Übergangszone zwischen dem Steppenland und dem kultivierten Land Syriens. Zwei Vegetationstypen sind hier zu unterscheiden: das landwirtschaftlich genutzte Land (Bewässerungsfelder oder Regenfeldfluren), das in dem westlichen und nordwestlichen Bereich dominiert, sowie das von natürlicher Vegetation bedeckte Land. Inselhaft befinden sich im westlichen Bereich unbewässerte Baumhaine (Ölbäume oder Pistazien). Häufiger treten hier bewässerte Felder von Weizen, Baumwolle oder Kartoffeln auf. Nach Osten sind Regenfelder von Gerste und manchmal Weizen zu finden. Oft existiert eine Mischung dieser beiden Arten in Form von Getreideregenfeldern, die im Winter zusätzliche Bewässerung empfangen, wenn Grundwasser vorhanden ist. Sommerkulturen wie Baumwolle und Gemüse erfordern intensive Bewässerung. Für die Bewässerung wurde das Grundwasser aus dem produktiven, salzigen, tiefen Grundwasserleiter der Kreide gepumpt oder aus dem weniger produktiven, mit besserer Qualität charakterisierten, oberen Grundwasserleiter des Paläogen. Die Brunnen des oberen Grundwasserleiters bewässern relativ kleine Felder, da sie meistens für die Wasserversorgung genutzt werden. Das Grundwasser aus dem tiefen Grundwasserleiter wird für die Bewässerung größerer Felder im Vergleich zu den oberen Grundwasserleiterbrunnen verwendet. Bewässerungsmethoden sind Überschwemmung von angelegten kleinen Becken und Sprengerbewässerung. Eine systematische Bewässerung wird nicht durchgeführt.

Der bewässerte Bereich wurde mittels der Satellitenbilder durch die Berechnung von dem NDVI (Normalised Difference Vegetation Index) klassifiziert. Dafür wurde die Satellitenaufnahme Landsat ETM+ vom 18.08.2000 verwendet.

Die NDVI Rasterdaten wurden in zehn nicht überwachte Klassen (unsupervised classification) gegliedert. Die Berechnung des NDVI ergab dieselben Reflexionswerte sowohl im Bereich der Palmyra-Ketten als auch der bewässerten Felder. Dieses Problem wurde durch die Überschneidung der berechneten Rasterdaten (NDVI) und des digitalen Höhenmodells mit der Entwicklung einer neuen Klassifikation gelöst. Dabei wurden die Bereiche über 500m Höhe, wie die Felder der natürlichen Vegetation, mit dem Wert Null und die landwirtschaftlich genutzten Felder mit dem Wert Eins gekennzeichnet. Diese 
neue Ebene wurde mit den zehn Klassen überschnitten, wodurch eine weitere neue Ebene erzeugt wurde, welche in drei Klassen untergliedert wurde (Abb. 7-12). Eine Klasse definiert die bewässerten Felder und wurde mit einem Gewicht von 3 gekennzeichnet. Eine andere Klasse legt die Regenfelder dar und wurde mit einem Gewicht von 4 gewertet. Die dritte Klasse bezeichnet das Land mit der natürlichen Vegetation und erhielt ein Gewicht von 5. Das Gewicht 3 wurde gegeben, weil die Felder mit Grundwasser aus dem tiefen Grundwasserleiter sowohl im Sommer als auch im Winter bewässert werden, wohingegen das Gewicht 4 Felder, die nur im Winter manchmal bewässert werden, aufzeigt. Diese Klassifikation wurde durch GPS Feldmessungen, die während der Feldarbeit, die am Ende des Sommer 2006 durchgeführt wurde, lokal nachgeprüft.

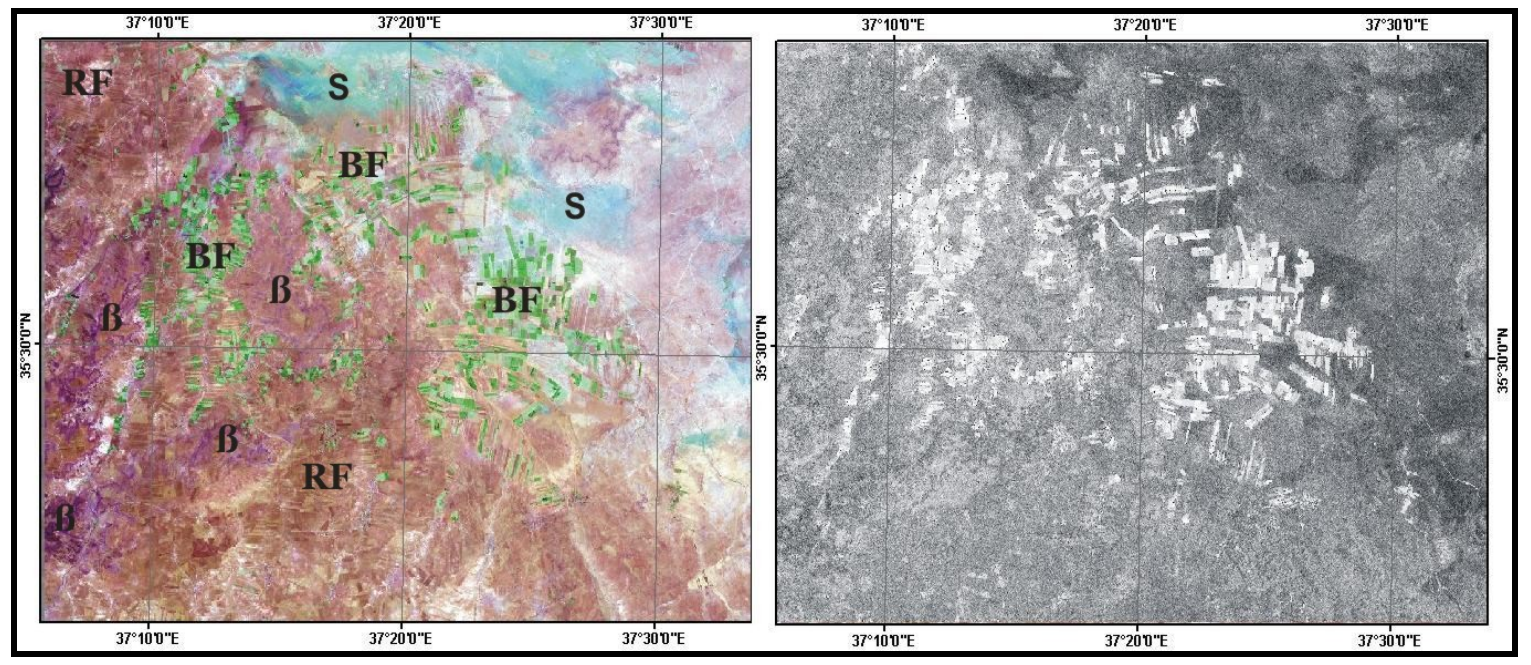

Abb. 7-11: Landsat 741 (RGB) Subszene (links), NDVI Bild (rechts). BF - bewässerte Felder, RF - Regenfelder, $S$ - versalzte Flächen, $ß$ - Basaltflächen 


\subsection{Zonen des Grundwasserneubildungspotentials}

Die Gesamtgewichte der finalen integrierten Rasterdaten wurden als Summe der Ebenengewichte abgeleitet. Diese wurde schließlich in fünf Klassen reklassifiziert (Abb. 7-12). Diese Methode basiert auf dem DRASTIC Verfahren von ALLER ET AL. (1985), welches für die Feststellung der Grundwasserverunreinigungszonen und -vulnerabilität (groundwater pollution and vulnerability) entwickelt wurde. Auf diese Weise bietet dieser integrierte Ansatz gegenüber der Hydrogeologie- und Zonensanierungkarte (Abb. 7-3), die keine direkten Informationen über das Grundwasserneubildungspotential Informationen enthält, eine Klassifikation verschiedener Zonen für das Grundwasserneubildungspotential.

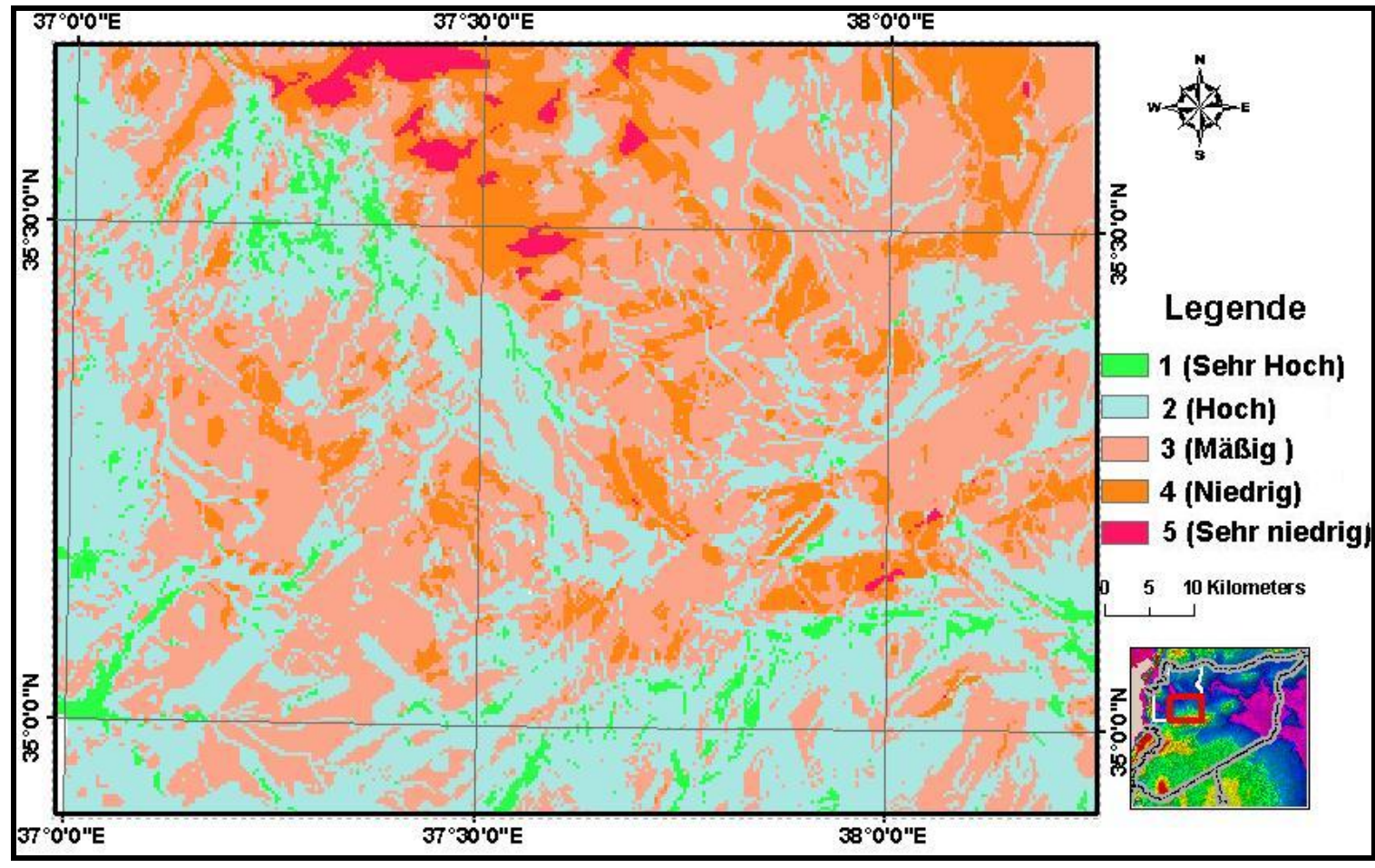

Abb. 7-12: Karte des Grundwasserneubildungspotentials

Eine dreidimensionale Darstellung wurde mittels ArcScene ausgearbeitet, wodurch die Beziehung zwischen der ermittelten Karte des Grundwasserneubildungspotenials und dem Untergrund klar dagelegt ist (Abb. 7-13). Die Gebiete mit dem höheren Potential für Grundwasserneubildung können in drei Bereichen untergeteilt werden. Der erste Bereich befindet sich am Rand der Palmyra-Ketten. Dabei handelt es sich um die Neubildung des tiefen Grundwasserleiters der Kreide. Der zweite Bereich liegt im Zentrum des Gebiets, und führt damit zur Speisen des oberen Grundwasserleiter in der Quartärablagerung. Der 
dritte Bereich ist auf dem Basalt-Plateau zu finden. Am Rand des Basalt-Plateaus lässt die Auswertung der Satellitenbilder und der Feldbeobachtung Grundwasservorkommen nach der Regenzeit erkennen.

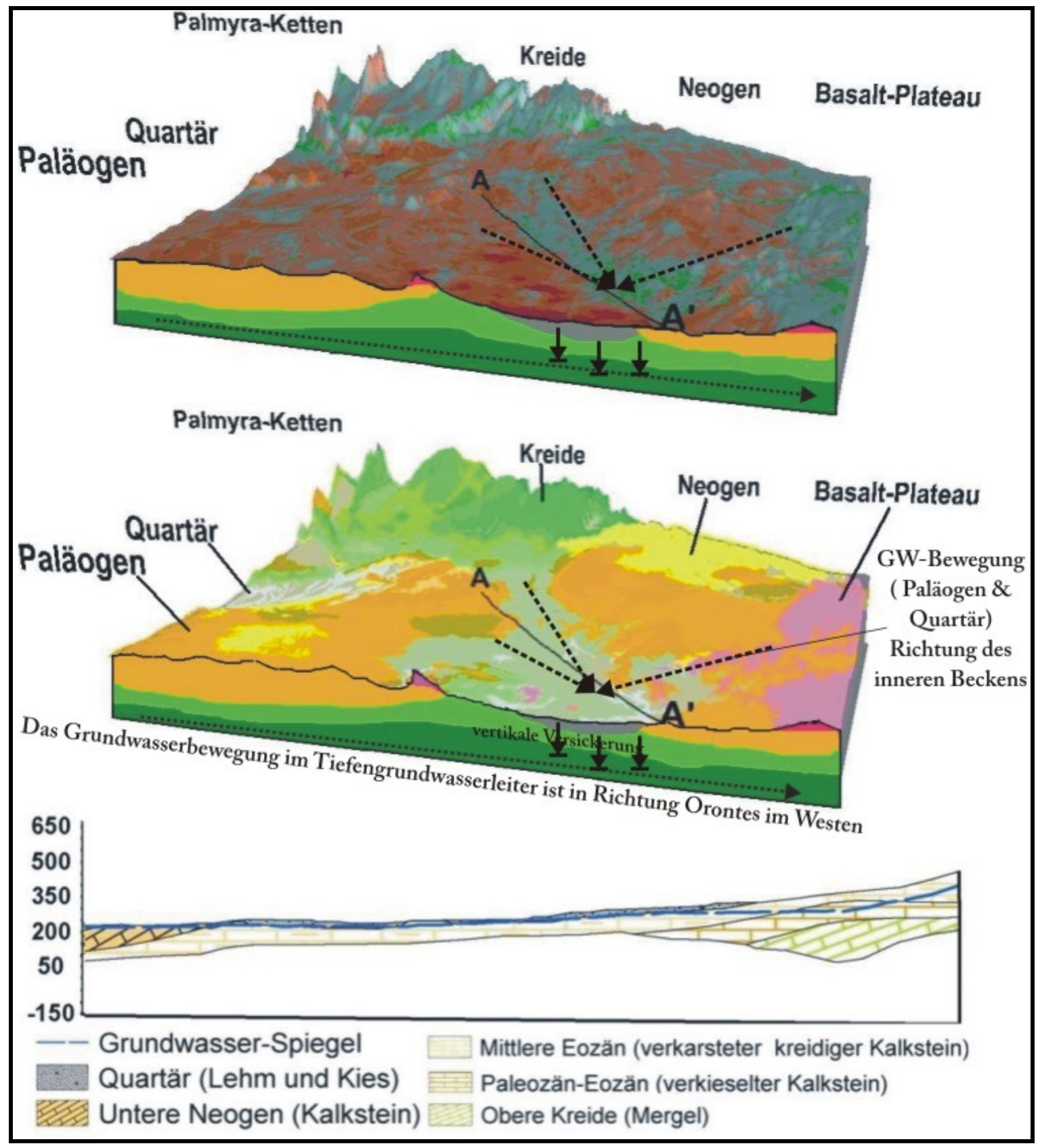

Abb. 7-13: Dreidimensionale Darstellung der Karte des Grundwasserneubildungspotentials und der Geologie. Unten ist ein Teil des Profils $X-X^{\prime}$ (Siehe Anhang 2) 


\subsection{I ntegration der Hydrochemiedaten}

Die Integration der Hydrochemiedaten in der Datenbank hat zum Ziel, die Einflüsse der Landoberfläche bzw. der geomorphologischen Prozesse auf die chemische Zusammensetzung des Grundwassers zu analysieren. Entsprechend beschäftigt sich die Arbeit mit dem oberen Grundwasserleiter. Dazu wurde die Karte des oberen Grundwasserleiters vor der Geländearbeit digitalisiert (Abb. 7-14), um die Definition der Probenahmepositionen zu unterstützen.

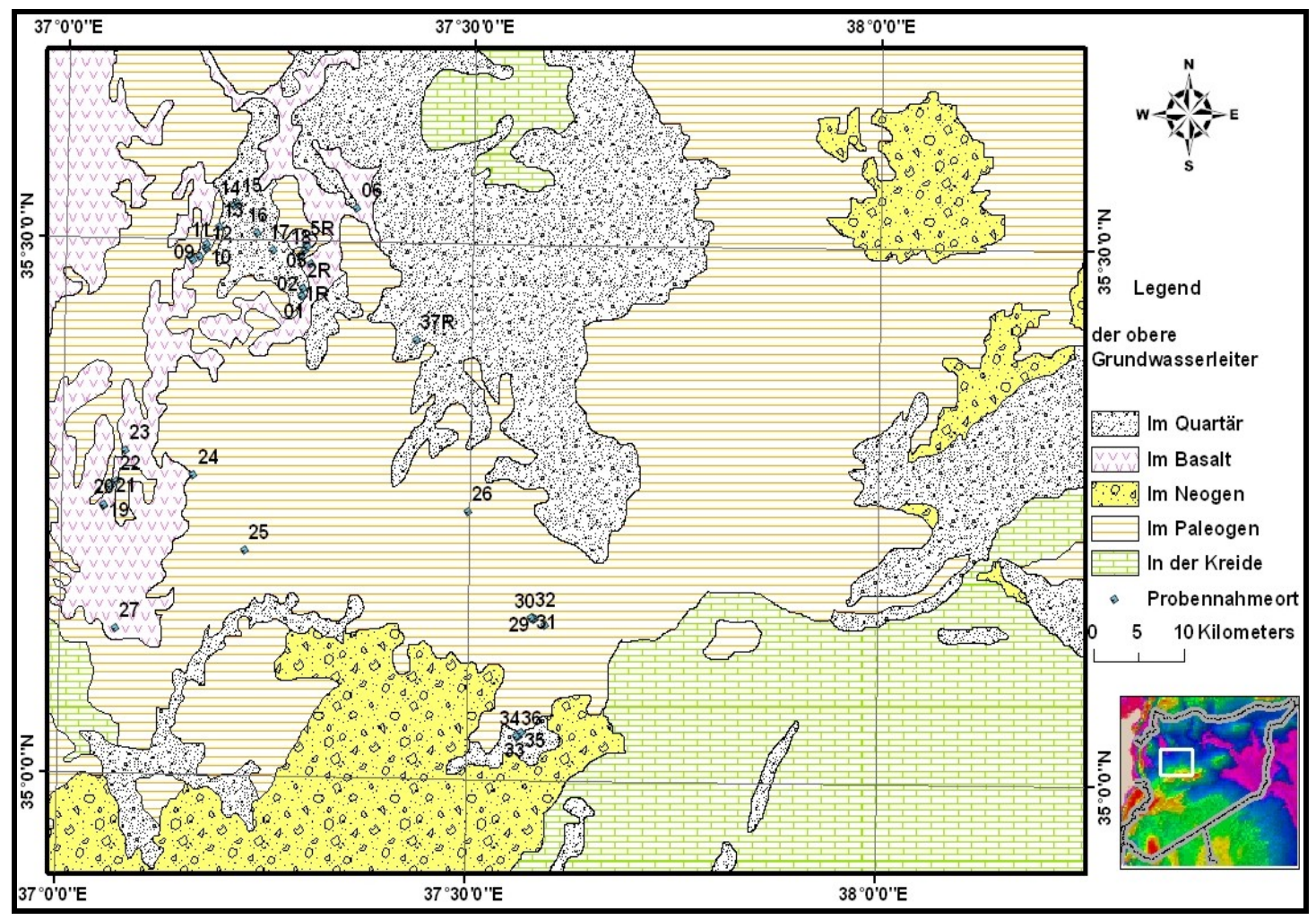

Abb. 7-14: Karte des oberen Grundwasserleiters

\subsubsection{Probenahme und Auswahl der Probenahmebereiche}

Die Auswahl der Bereiche bzw. Positionen der Probenahme verlangt eine Gliederung des Zielgebiets in Teileinzugsgebiete. Eine Karte für die hydrologischen Teileinzugsgebiete wurde mittels ArcHydro erstellt und darauf folgend editiert. Die Editierung war unerlässlich, weil das Verhältnis der natürlichen Teileinzugsgebiete wegen der anthropogenen Aktivitäten (das Einrichten von kleinen Staudämmen oder Water Harvesting) geändert wurde. Die Editierung wurde durch die Kombination der erstellten thematischen Teileinzugsgebietskarte mit den Satellitenbildern durchgeführt. Für die Bestimmung der Positionen kleiner Staudämme wurden die zur Verfügung stehenden aktuellen ASTER Szenen 
vom 28.03.2005 und 18.10.2003 verwendet. Die vorhandenen Landsatbilder waren für dieses Ziel nicht geeignet, da einige Staudämme neu errichtet wurden (Abb. 7-15).

Unter Berücksichtigung aller Umstände, die durch die oben dargestellten Arbeitschritte nachzuvollziehen sind, wurden zwei Bereiche für die Grundwasserprobenahme definiert. Ein Bereich liegt direkt am Fuß der Palmyra-Ketten (Teileinzugsgebiet A). Hier sind die anthropogenen Aktivitäten sehr begrenzt. Der zweite Teil liegt im Westen und schließt ein Teileinzugsgebiet innerhalb des Basalts ein (Teileinzugsgebiet B).

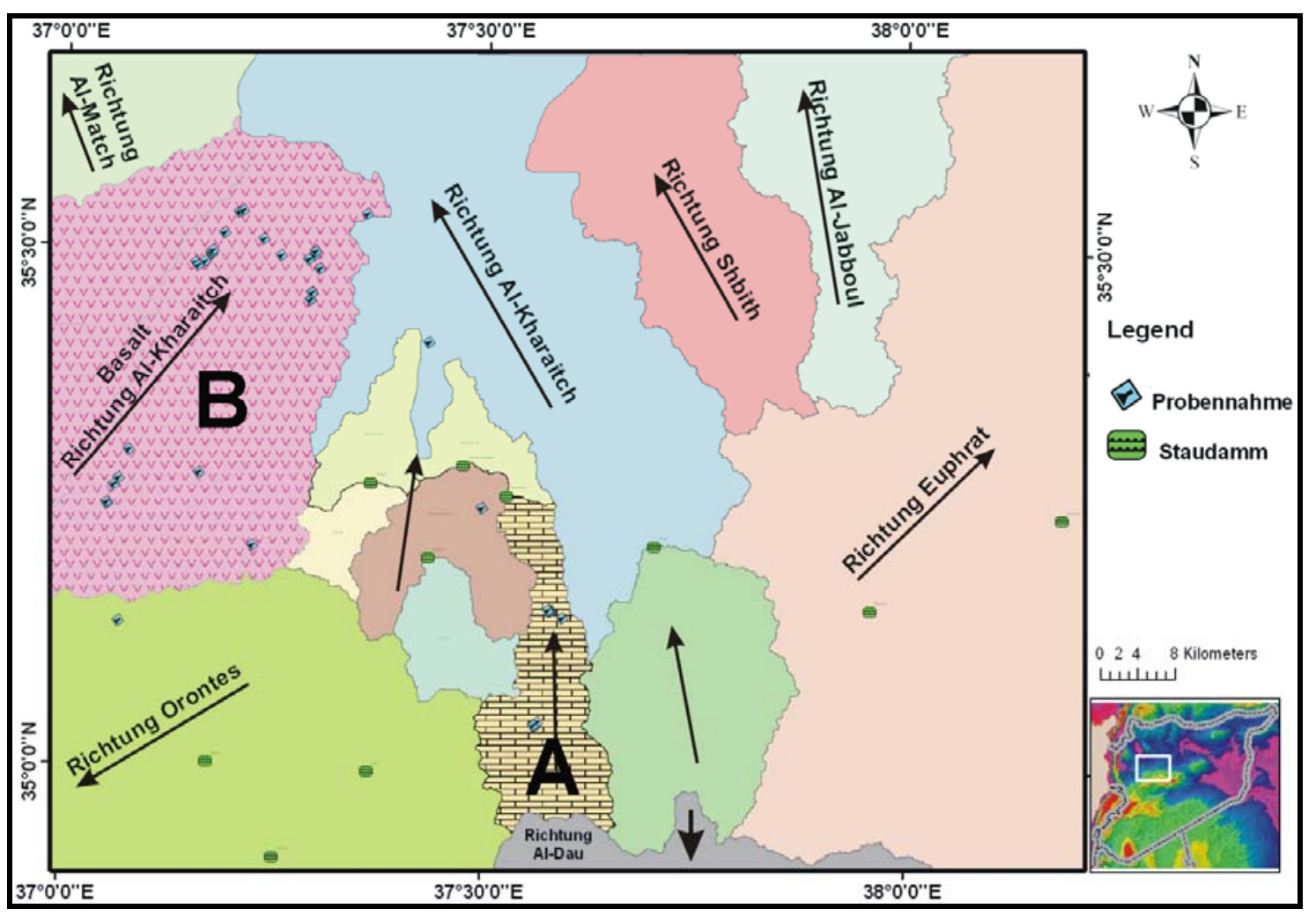

Abb. 7-15: Teileinzugsgebiete A \& B und die Wasserabflussrichtung (Pfeile)

Die Geländearbeit wurde am Ende der Sommerzeit und Anfang der Winterzeit 2006 vollzogen. Dabei wurden 36 Proben während des Sommers und vier Stichproben nach dem ersten Regenereignis genommen. Nach Angabe der Brunneninhaber wird das Grundwasser aus Kalkstein- oder kreidigen Kalksteinschichten des Paläogens gepumpt. Der Flurabstand der Brunnen variiert zwischen 40 und 70 Meter. Im Feld wurden die pH-Werte des Grundwassers gemessen. Alle Proben waren relativ homogen im $\mathrm{pH}$-Wert $(6,7-7,8)$. Für die Beobachtung des Salzgehalts wurde ebenfalls im Feld die elektrische Leitfähigkeit (EC) gemessen. 


\subsubsection{Klassifikation und Hydrochemie des Grundwassers}

Die Leitfähigkeit eignet sich zwar als guter Parameter zu Abschätzung des Gesamtlösungsinhalts, jedoch ist eine Einzelstoffanalytik wesentlich aussagefähiger in Bezug auf die Typisierung von Wässern, sowie zur Erkennung von Prozessen. Daher wurden parallel zu den Leitfähigkeitsmessungen Proben (10 mL, keine Konservierung) entnommen und nach Göttingen in die Abteilung der angewandten Geologie zur Analytik gebracht. Die Proben wurden vor der Messung gefiltert $(<0.45 \mu \mathrm{m})$ und entsprechend dem linearen Arbeitsbereich verdünnt. An- und Kationen wurden parallel auf getrennten Ionenchromatographen analysiert. Für die Kationenbestimmung $(\mathrm{Na}+, \mathrm{K}+, \mathrm{Mg} 2+, \mathrm{Ca} 2+)$ wurde ein isokratisches System (DIONEX DX-500) bei einer Temperatur von $42^{\circ} \mathrm{C}$ mit einer CS16 (3*250mm) Trennsäule und Vorsäule sowie $35 \mathrm{mM}$ Methansulfonsäure mit einer Flussrate von $0,38 \mathrm{ml} / \mathrm{min}$ als Eluent verwendet. Die Detektion erfolgte über Leitfähigkeitsdetektion in Kombination mit der Suppressortechnik. Die Proben wurden über einen Autosampler mittels Vollschleifeninjektion (Probenschleife 5 $\mu$ l) aufgegeben. Der kalibrierte lineare Arbeitsbereich lag für Natrium zwischen 50 - 120mg/l, für Kalium zwischen 1-3 mg/l und für Magnesium sowie Calcium zwischen 10 -100 mg/l. Die Proben wurden so verdünnt das die individuellen Konzentrationen innerhalb der Kalibration lagen.

Für die Anionenbestimmung (F-, Cl-, NO3-, SO42-) wurde ein isokratisches System (DIONEX DX-320) mit $\mathrm{KOH}$ Eluentengenerator bei einer Temperatur von $30^{\circ} \mathrm{C}$ mit einer AS11-HC $(2 * 250 \mathrm{~mm})$ Trennsäule und Vorsäule sowie $23 \mathrm{mM} \mathrm{KOH}$ mit einer Flussrate von $0,38 \mathrm{ml} / \mathrm{min}$ als Eluent verwendet. Die Detektion erfolgte über Leitfähigkeitsdetektion in Kombination mit der Suppressortechnik. Die Proben wurden über einen Autosampler mittels Vollschleifeninjektion (Probenschleife 5 $\mu$ l) aufgegeben. Der kalibrierte lineare Arbeitsbereich lag für Fluorid zwischen1-10 mg/l, für Chlorid, Sulfat sowie Nitrat zwischen $1-150 \mathrm{mg} / \mathrm{l}$. Die Proben wurden so verdünnt, dass die individuellen Konzentrationen innerhalb der Kalibration lagen. Die Konzentration von Hydrogenkarbonat $\left(\mathrm{HCO}_{3}{ }^{-}\right)$konnte im Feld nicht bestimmt werden und wurde als Differenz zur Kationenequivalenzsumme abgeschätzt. Damit sind alle gebildeten Ionenbilanzen ausgeglichen.

Die Angaben für den Gesamtlösungsinhalt TDS (total dissolved solids) ergeben sich als Summe der Einzelionen, bestimmt mittels Ionenchromatographie, und der über Differenzverfahren ermittelten Hydrogencarbonatkonzentration. Die Ergebnisse zeigen, dass 
mehr als 80\% der Proben nach CARROLL (1962) als Frischgrundwasser einzustufen sind (Tab. 7-5). Allerdings liegen viele Werte an der Obergrenze indem sie den Gesamtlösungsinhalt von 900 mg/l (Tab. 7-6) überschreiten. Der TDS nimmt in Richtung Nordosten und Osten zu (Abb. 7-16). Dieses Ergebnis stimmt mit der Geomorphologie überein, weil die Salzflächen sich im Nordosten des Untersuchungsgebiets befinden.

Der TDS steht mit der elektrischen Leitfähigkeit (EC) in Beziehung, da die gelösten Feststoffe in der Flüssigkeit dem Salzgehalt entsprechen und diese die Leitfähigkeit im Wasser ausmachen. Die Darstellung für den TDS zu der elektrischen Leitfähigkeit (Abb. 7-16) vermittelt eine Vorstellung über den Salzgehalt. Diese Beziehung lässt sich durch die Näherungsgleichungen erkennen. Für Süßwasser gilt in etwa die Näherung: (TDS $\mathrm{mg} / \mathrm{L}=0,5 * \mathrm{EC}$ in $\mu \mathrm{S} / \mathrm{cm}$ ) wobei die Konstante mit steigendem Salzgehalt auf bis zu 0,9 ansteigt.

Tab. 7-5: Allgemeine Klassifikation des Grundwassers (CARROLL, 1962)

\begin{tabular}{|c|c|}
\hline Kategorie des Wassers & TDS (mg/L) \\
\hline Frisch & $0-1000$ \\
\hline Brackig & $1000-10000$ \\
\hline salzig & $10000-100000$ \\
\hline Sehr Salzig & $>100000$ \\
\hline
\end{tabular}

Tab. 7-6: Gesamtlösungsinhalt TDS der analysierten Grundwasserproben

\begin{tabular}{|c|c|c|c|c|c|c|c|}
\hline \multirow{2}{*}{ Probe } & \multirow{2}{*}{ TDS } & \multirow{2}{*}{ Probe } & TDS & \multirow{2}{*}{ Probe } & TDS & \multirow{2}{*}{ Probe } & TDS \\
& $\mathbf{m g} / \mathbf{l}$ & & $\mathbf{m g} / \mathbf{l}$ & & $\mathbf{m g} / \mathbf{l}$ & & $\mathbf{m g} / \mathbf{l}$ \\
\hline $\mathbf{1}$ & 546.1 & $\mathbf{1 0}$ & 944.0 & $\mathbf{1 9}$ & 402.0 & $\mathbf{2 8}$ & 1429.4 \\
\hline $\mathbf{2}$ & 796.5 & $\mathbf{1 1}$ & 925.2 & $\mathbf{2 0}$ & 359.7 & $\mathbf{2 9}$ & 790.2 \\
\hline $\mathbf{3}$ & 647.0 & $\mathbf{1 2}$ & 925.4 & $\mathbf{2 1}$ & 436.3 & $\mathbf{3 0}$ & 798.4 \\
\hline $\mathbf{4}$ & 656.2 & $\mathbf{1 3}$ & 1366.5 & $\mathbf{2 2}$ & 420.3 & $\mathbf{3 1}$ & 1390.1 \\
\hline $\mathbf{5}$ & 787.7 & $\mathbf{1 4}$ & 4667.9 & $\mathbf{2 3}$ & 491.8 & $\mathbf{3 2}$ & 1028.2 \\
\hline $\mathbf{6}$ & 4425.3 & $\mathbf{1 5}$ & 4261.7 & $\mathbf{2 4}$ & 492.3 & $\mathbf{3 3}$ & 520.7 \\
\hline $\mathbf{7}$ & 643.3 & $\mathbf{1 6}$ & 1850.4 & $\mathbf{2 5}$ & 515.4 & $\mathbf{3 4}$ & 512.7 \\
\hline $\mathbf{8}$ & 821.1 & $\mathbf{1 7}$ & 912.7 & $\mathbf{2 6}$ & 723.1 & $\mathbf{3 5}$ & 531.3 \\
\hline $\mathbf{9}$ & 960.5 & $\mathbf{1 8}$ & 820.3 & $\mathbf{2 7}$ & 402.2 & $\mathbf{3 6}$ & 540.2 \\
\hline
\end{tabular}




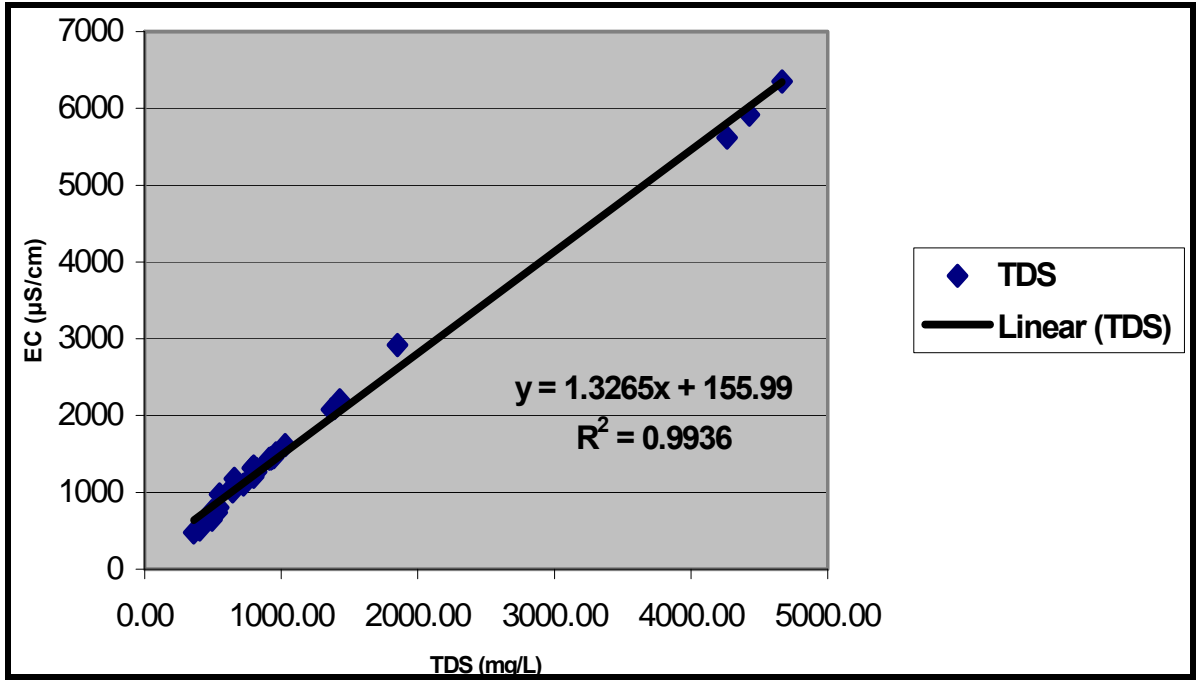

Abb. 7-16: Beziehung zwischen der Leitfähigkeit \& dem TDS des Grundwassers

Zur übersichtlichen Klassifizierung der Grundwässer wurden deren Analysenergebnisse mittels AquaChem v.5.0 in einem Piperdiagramm dargestellt (Abb. 7-17). Danach lässt sich das Grundwasser in zwei Gruppen untergliedern:

1. Grundwasser mit niedrigem salzigem Gehalt vom Kalzium-Natrium-Magnesium Bikarbonat-Typ mit folgenden Ionenreihenfolge: $\mathrm{Ca}^{2+}>\mathrm{Na}^{+}>\mathrm{Mg}^{2+}>\mathrm{K}^{+}$and $\mathrm{HCO}_{3}>$ $\mathrm{Cl}^{-}>\mathrm{SO}_{4}{ }^{2-}$.

2. eher brackiges Grundwasser in folgenden Brunnen: $6\left(\mathrm{Na}-\mathrm{Ca}^{2+}-\mathrm{SO}_{4}{ }^{2-} \mathrm{Cl}^{-}\right), 14(\mathrm{Na}-$ $\left.\mathrm{Mg}^{2+}-\mathrm{Ca}^{2+}-\mathrm{SO}_{4}{ }^{2-} \mathrm{Cl}^{-}\right), 15\left(\mathrm{Ca}^{2+}-\mathrm{Mg}^{2+}-\mathrm{Na}^{+}-\mathrm{SO}_{4}{ }^{2-}-\mathrm{Cl}^{-}\right)$und $16\left(\mathrm{Ca}^{2+}-\mathrm{Na}^{+}-\mathrm{Mg}^{2+}-\mathrm{Cl}^{-}-\right.$ $\left.\mathrm{SO}_{4}{ }^{2-}\right)$, die im Nordosten des Bereichs liegen.

In der Abbildung (7-17) ist die Karte der geographischen Positionen neben dem Piperdiagramm dargestellt. Die zwei genannten Gruppen wurden in den Farben rot und blau definiert. Eine dritte Gruppe, die in der s. z. Umwandlungszone zu gliedern ist, lässt sich auch erkennen. Die Tabelle (7-7) zeigt die Wassertypen aller betrachteten Brunnen des Paläogens sowie die Brunnen SP11, SP12 und SP13 der Kreide. 


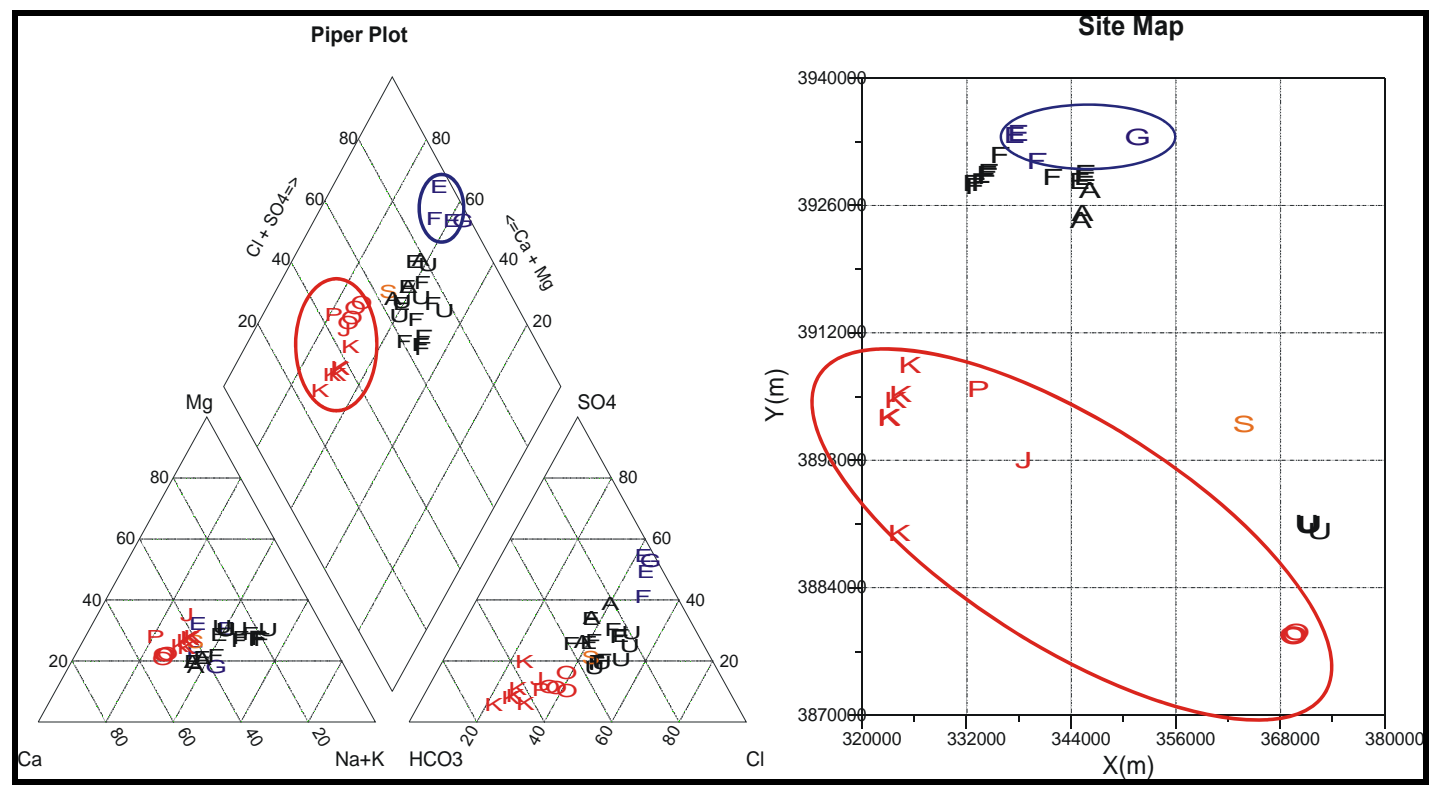

Abb. 7-17: Piperdiagramm (links) und die Karte der Probenamepositionen (rechts)

Die geographische Verteilung der ersten Gruppe stimmt mit der erstellten Karte des Grundwasserneubildungspotentials überein. Der Salzgehalt ist bei den möglichen Grundwasserneubildungszonen sehr gering und nimmt in Richtung der Ausflusszonen zu. 
Tab. 7-7: Die Wassertypen der betrachteten Brunnen

\begin{tabular}{|c|c|c|c|c|c|c|c|c|c|}
\hline Br.-Nr. & $\begin{array}{c}\text { Wasser- } \\
\text { Type }\end{array}$ & $\begin{array}{l}\text { TDS } \\
\mathrm{mg} / \mathrm{l}\end{array}$ & $\begin{array}{l}\mathrm{Na}^{+} \\
\mathrm{mg} / \mathrm{l}\end{array}$ & $\begin{array}{l}\mathrm{Ca}^{2+} \\
\mathrm{mg} / \mathrm{l}\end{array}$ & $\begin{array}{l}\mathrm{Mg}^{2+} \\
\mathrm{mg} / \mathrm{l}\end{array}$ & $\begin{array}{c}\mathrm{Cl}^{-} \\
\mathrm{mg} / \mathrm{l}\end{array}$ & $\begin{array}{l}\mathrm{SO}_{4}{ }^{2-} \\
\mathrm{mg} / \mathrm{l}\end{array}$ & $\begin{array}{c}\mathrm{HCO}_{3}^{-} \\
\mathrm{mg} / \mathrm{l}\end{array}$ & $\begin{array}{l}\mathrm{NO}_{3}^{-} \\
\mathrm{mg} / \mathrm{l}\end{array}$ \\
\hline SP11 & $\mathrm{Ca}-\mathrm{Mg}-\mathrm{SO} 4$ & 2731 & 109.7 & 421.0 & 197.6 & 177.3 & 1611.9 & 207.4 & 6.2 \\
\hline SP12 & $\mathrm{Ca}-\mathrm{Mg}-\mathrm{SO} 4$ & 2376 & 80.0 & 336.1 & 202.1 & 187.9 & 1354.3 & 213.5 & 2.0 \\
\hline SP13 & $\mathrm{Ca}-\mathrm{Mg}-\mathrm{SO} 4-\mathrm{Cl}$ & 2458 & 124.0 & 350.4 & 192.9 & 319.2 & 1281.9 & 189.1 & 0.5 \\
\hline 1 & $\mathrm{Ca}-\mathrm{Na}-\mathrm{Mg}-\mathrm{Cl}-\mathrm{HCO} 3-\mathrm{SO} 4$ & 546 & 62.8 & 70.6 & 18.7 & 82.0 & 76.5 & 135.8 & 63.5 \\
\hline 2 & $\mathrm{Ca}-\mathrm{Na}-\mathrm{Cl}-\mathrm{SO} 4$ & 796 & 98.4 & 105.5 & 25.1 & 130.5 & 171.6 & 123.5 & 99.4 \\
\hline 3 & $\mathrm{Ca}-\mathrm{Na}-\mathrm{Mg}-\mathrm{Cl}-\mathrm{SO} 4-\mathrm{HCO} 3$ & 647 & 80.7 & 78.2 & 23.5 & 98.7 & 124.1 & 138.5 & 81.3 \\
\hline 4 & $\mathrm{Ca}-\mathrm{Na}-\mathrm{Mg}-\mathrm{Cl}-\mathrm{SO} 4-\mathrm{HCO} 3$ & 656 & 81.7 & 79.3 & 23.8 & 99.7 & 125.3 & 142.2 & 82.2 \\
\hline 5 & $\mathrm{Ca}-\mathrm{Na}-\mathrm{Mg}-\mathrm{Cl}-\mathrm{SO} 4$ & 788 & 94.0 & 105.0 & 27.8 & 157.2 & 124.0 & 134.5 & 94.8 \\
\hline 6 & $\mathrm{Na}-\mathrm{Ca}-\mathrm{SO} 4-\mathrm{Cl}$ & 4425 & 670.2 & 549.3 & 149.3 & 994.4 & 1612.8 & 134.2 & 674.2 \\
\hline 7 & $\mathrm{Na}-\mathrm{Ca}-\mathrm{Mg}-\mathrm{HCO} 3-\mathrm{Cl}-\mathrm{SO} 4$ & 643 & 97.0 & 51.5 & 29.6 & 101.2 & 99.2 & 198.5 & 98.3 \\
\hline 8 & $\mathrm{Na}-\mathrm{Mg}-\mathrm{Ca}-\mathrm{Cl}-\mathrm{HCO} 3-\mathrm{SO} 4$ & 821 & 126.7 & 65.4 & 40.7 & 162.2 & 141.1 & 222.8 & 128.7 \\
\hline 9 & $\mathrm{Na}-\mathrm{Mg}-\mathrm{Ca}-\mathrm{Cl}-\mathrm{HCO} 3$ & 960 & 166.4 & 63.5 & 47.6 & 222.8 & 126.2 & 272.1 & 168.4 \\
\hline 10 & $\mathrm{Na}-\mathrm{Mg}-\mathrm{Ca}-\mathrm{Cl}-\mathrm{HCO} 3$ & 944 & 162.9 & 63.1 & 45.6 & 212.2 & 118.7 & 284.7 & 165.2 \\
\hline 11 & $\mathrm{Na}-\mathrm{Mg}-\mathrm{Ca}-\mathrm{Cl}-\mathrm{HCO} 3$ & 925 & 160.6 & 59.5 & 45.3 & 205.4 & 120.2 & 275.8 & 163.4 \\
\hline 12 & $\mathrm{Na}-\mathrm{Mg}-\mathrm{Ca}-\mathrm{Cl}-\mathrm{HCO} 3$ & 925 & 164.2 & 58.0 & 44.5 & 207.2 & 118.2 & 278.4 & 166.5 \\
\hline 13 & $\mathrm{Na}-\mathrm{Mg}-\mathrm{Ca}-\mathrm{Cl}-\mathrm{SO} 4-\mathrm{HCO} 3$ & 1367 & 227.5 & 96.8 & 72.3 & 301.0 & 273.0 & 289.4 & 228.9 \\
\hline 14 & $\mathrm{Na}-\mathrm{Mg}-\mathrm{Ca}-\mathrm{SO} 4-\mathrm{Cl}$ & 4668 & 674.6 & 443.3 & 273.3 & 1118.5 & 1657.6 & 255.8 & 683.1 \\
\hline 15 & $\mathrm{Ca}-\mathrm{Mg}-\mathrm{Na}-\mathrm{SO} 4-\mathrm{Cl}$ & 4262 & 470.7 & 505.2 & 261.3 & 913.5 & 1603.1 & 155.9 & 477.7 \\
\hline 16 & $\mathrm{Ca}-\mathrm{Na}-\mathrm{Mg}-\mathrm{Cl}-\mathrm{SO} 4$ & 1850 & 221.5 & 241.3 & 76.7 & 391.7 & 445.1 & 150.7 & 223.7 \\
\hline 17 & $\mathrm{Na}-\mathrm{Ca}-\mathrm{Mg}-\mathrm{Cl}-\mathrm{SO} 4-\mathrm{HCO} 3$ & 913 & 129.4 & 101.7 & 35.4 & 197.6 & 156.5 & 176.6 & 130.8 \\
\hline 18 & $\mathrm{Na}-\mathrm{Ca}-\mathrm{Mg}-\mathrm{Cl}-\mathrm{HCO} 3-\mathrm{SO} 4$ & 820 & 107.4 & 81.4 & 42.7 & 167.9 & 147.9 & 251.2 & 111.5 \\
\hline 19 & $\mathrm{Ca}-\mathrm{Na}-\mathrm{Mg}-\mathrm{HCO} 3-\mathrm{Cl}$ & 402 & 38.5 & 47.2 & 18.4 & 41.4 & 45.6 & 170.7 & 39.2 \\
\hline 20 & $\mathrm{Ca}-\mathrm{Na}-\mathrm{Mg}-\mathrm{HCO} 3-\mathrm{Cl}$ & 360 & 33.1 & 43.3 & 15.7 & 38.3 & 15.5 & 172.9 & 33.6 \\
\hline 21 & $\mathrm{Ca}-\mathrm{Na}-\mathrm{Mg}-\mathrm{HCO} 3-\mathrm{Cl}$ & 436 & 42.4 & 51.1 & 20.0 & 59.8 & 15.0 & 212.6 & 43.1 \\
\hline 22 & $\mathrm{Ca}-\mathrm{Na}-\mathrm{Mg}-\mathrm{HCO} 3-\mathrm{Cl}$ & 420 & 40.7 & 47.7 & 19.3 & 45.6 & 25.2 & 190.7 & 41.6 \\
\hline 23 & $\mathrm{Ca}-\mathrm{Na}-\mathrm{Mg}-\mathrm{HCO} 3-\mathrm{Cl}$ & 492 & 47.4 & 60.4 & 19.7 & 56.1 & 23.7 & 238.2 & 48.5 \\
\hline 24 & $\mathrm{Ca}-\mathrm{Mg}-\mathrm{Na}-\mathrm{HCO} 3-\mathrm{Cl}$ & 492 & 30.4 & 72.3 & 23.0 & 61.9 & 26.4 & 186.7 & 31.1 \\
\hline 25 & $\mathrm{Ca}-\mathrm{Mg}-\mathrm{Na}-\mathrm{HCO} 3-\mathrm{Cl}$ & 515 & 42.9 & 56.6 & 31.0 & 67.8 & 41.2 & 209.9 & 44.8 \\
\hline
\end{tabular}




\subsubsection{Hydrochemie des Teileinzugsgebiets A (Palmyra-Ketten)}

Das Teileinzugsgebiet hat eine Größe von $286.14 \mathrm{~km}^{2}$. Die Lithologie der Oberfläche in diesem Teileinzugsgebiet ist unterschiedlich (Abb. 7-18). Im Norden besteht die Region aus Mergel und kreidigen Kalkstein des Paläogens und im Süden aus dem massiven oder laminaren Kalkstein sowie Dolomit und selten Gips der Kreide. In der Mitte ist das Paläogen von den Konglomeraten des Neogens abgedeckt (Abb. 7-19).

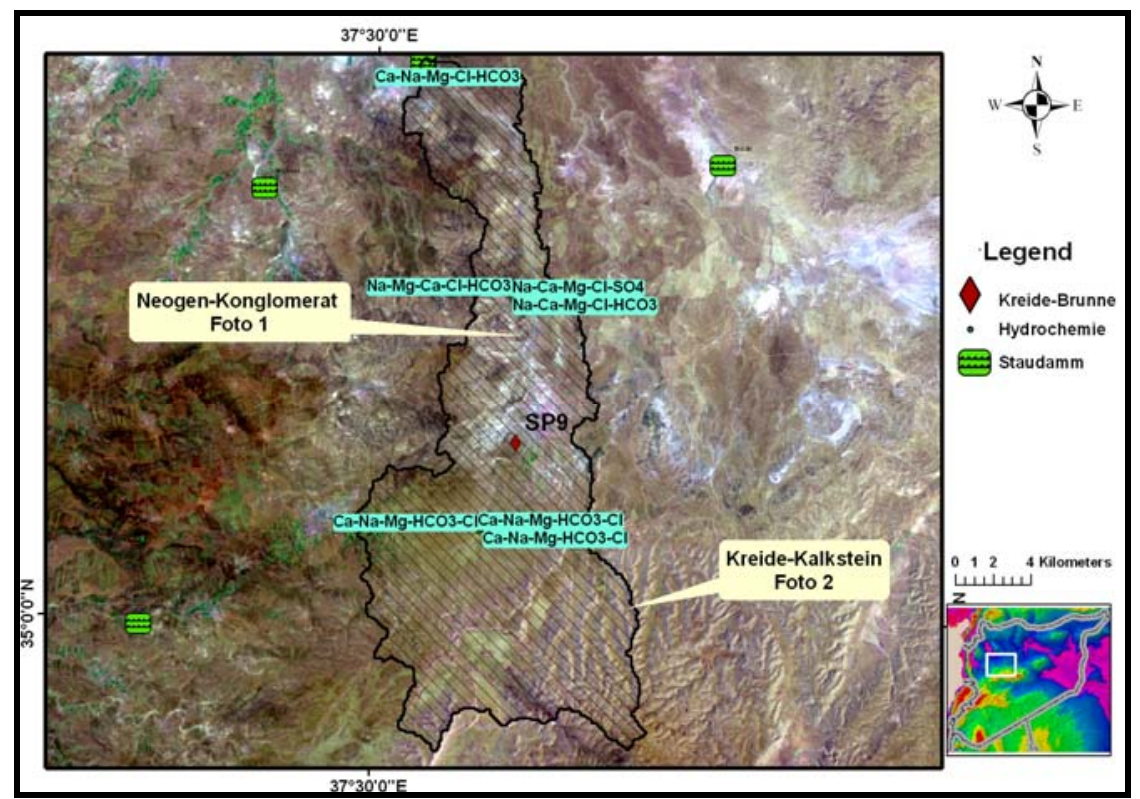

Abb. 7-18: Das Teileinzugsgebiet A

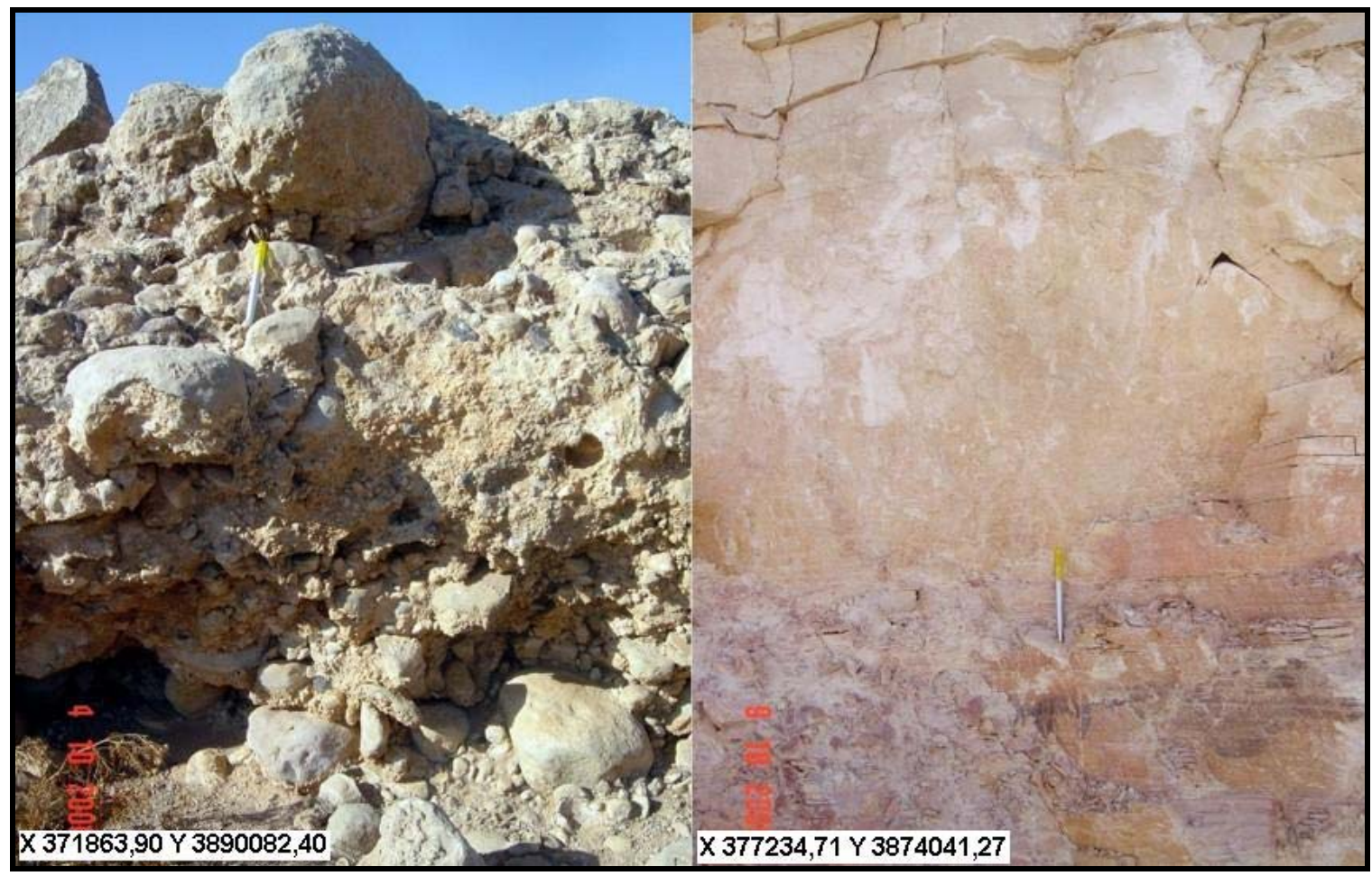

Abb. 7-19: Das Konglomerat des Neogen (links) und Kalkstein der Kreide (rechts) 
Das Grundwasser in dem höher gelegenen Bereich dieses Teileinzugsgebiets ist Frischwasser (Bikarbonat) mit der Ionenreihenfolge $\mathrm{Ca}^{2+}>\mathrm{Na}^{+}>\mathrm{Mg}^{2+}$ sowie $\mathrm{HCO}_{3}{ }^{-}>\mathrm{Cl}^{-}>$ $\mathrm{SO}_{4}{ }^{2-}$. Nach Norden hin ändert sich die Hydrochemie deutlich und die Ionenreihenfolge ändert sich $\mathrm{zu} \mathrm{Na}{ }^{+}>\mathrm{Ca}^{2+} / \mathrm{Mg}^{2+}>\mathrm{Mg}^{2+} / \mathrm{Ca}^{2+}$ sowie $\mathrm{Cl}^{-}>\mathrm{SO}_{4}{ }^{2-} / \mathrm{HCO}_{3}{ }^{-}>\mathrm{HCO}_{3}{ }^{-} / \mathrm{SO}_{4}{ }^{2-}$. Diese lässt sich wie folgt erklären: Die erste Gruppe befindet sich direkt am Rand der Palmyra-Ketten, wo die oberen Grundwasserleiter Quartär und Neogen sind. Diese beiden Grundwasserleiter liegen topographisch höher als das Paläogen. Das von der Oberfläche im Winter infiltrierte Wasser versickert in den Grundwasserleiter Quartär und Neogen und erreicht entlang der Klüfte den paläogenen Grundwasserleiter. Das Regenwasser fließt in den Wadis nach Norden in Richtung der zweiten Gruppe. Auf dem Weg löst das Wasser die Verwitterungsprodukte. Das Wasser infiltriert langsam und verursacht eine hohe Ionenkonzentration in dem Grundwasserleiter des Paläogens (Abb. 720).

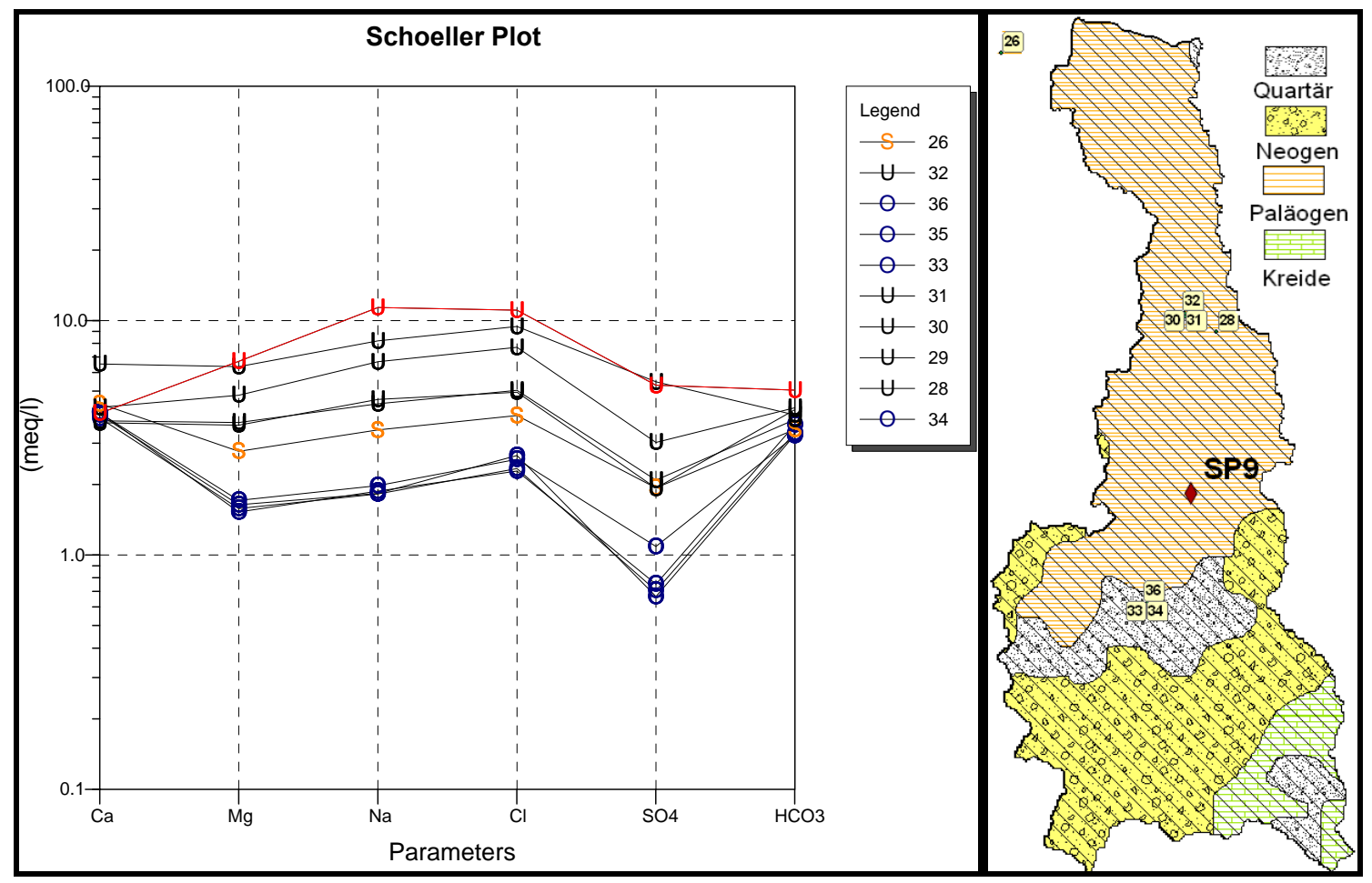

Abb. 7-20: Das Schöllerdiagramm (links) und Probenpositionen (Basis ist Grundwasserleiterkarte(rechts) 
Im Brunnen 28 fällt ein sehr deutlich erhöhter Gesamtlösungsinhalt (TDS) auf (Abb. 720). Dieser lässt sich durch die Wasserinfiltration aus dem trocknen Wadi in unmittelbarer Umgebung erklären. Die Brunnen 29, 30, 31, 32 und 33 liegen in der gleichen topographischen Höhe und besitzen eine identische Tiefe. Dennoch hat der Brunnen $31 \mathrm{im}$ Vergleich einen relativ hohen Gesamtlösungsinhalt. Da kein Wadi direkt neben den genannten Brunnen liegt, wurde versucht, die Situation im Hinblick auf eine mögliche Verbindung mit dem tiefen Grundwasserleiter zu erklären. Allerdings negiert der direkte Vergleich der Daten diese Annahme (Abb. 7-21), weil der Gesamtlösungsinhalt in dem Brunnen SP9 vergleichsweise geringer ausfällt. Diese Beobachtung bezieht sich mit groBer Wahrscheinlichkeit, wie bei dem Brunnen 28 auf eine Infiltration aus einem nicht weit entfernten Wadi. Dieser Brunnen ist möglicherweise in einer Verkarstungszone angesiedelt, welche eine hydraulische Anbindung an das Wadi haben könnte. Darauf deutet zusätzlich der hohe Kalziumgehalt hin (rot markiert in der Abb. 7-21).

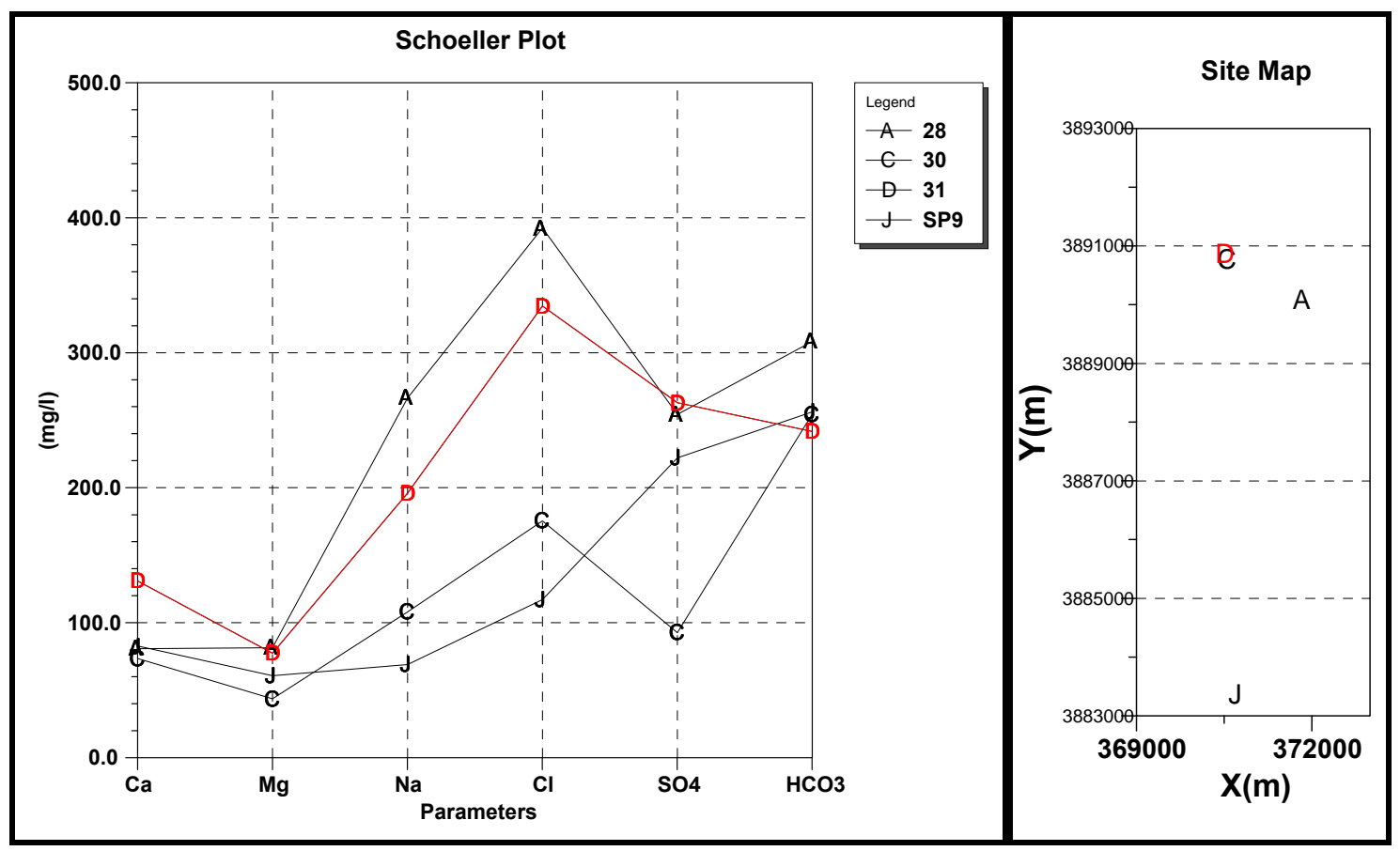

Abb. 7-21: Schöllerdiagramm der Brunnen 28, 30, 31 und SP9 (links) sowie deren geographischen Positionen (rechts) 


\subsubsection{Hydrochemie des Teileinzugsgebiets B (Basaltregion)}

Das Teileinzugsgebiet B hat eine Größe von ca. $1046 \mathrm{~km}^{2}$. In dem westlichen Teil des Teileinzugsgebiets stellt sich die Hydrochemie sehr deutlich als Abbild der geomorphologischen Prozesse bzw. Einflüsse dar. In dem höher gelegenen Bereich ist die Ionenreihenfolge $\mathrm{Ca}^{2+}>\mathrm{Na}^{+}>\mathrm{Mg}^{2+}$ oder $\mathrm{Ca}^{2+}>\mathrm{Mg}^{2+}>\mathrm{Na}^{+}$. Diese relative Änderung lässt sich z.B. durch die Verwitterung von Basalt in Na-reiche Produkte erklären. Im Osten des Gebietes führt die Lösung von kreidigen Kalkstein des Paläogens zu einem erhöhten $\mathrm{Mg}^{2+}$-Gehalt im Grundwasser (Abb. 7-22). Nach Norden, wo das Ausflussgebiet von Sabkha Al-Kharaitch ist, nehmen die $\mathrm{Cl}^{-}$und $\mathrm{SO}_{4}{ }^{2-}$ Ionenkonzentration zu. Die Landoberfläche von dem Sabkha ist zu einem großen Prozentteil von Salzstein und Gips bedeckt. Deswegen ist die Ionenreihenfolge in mehreren Brunnen $\mathrm{Na}^{+}>\mathrm{Ca}^{2+}>\mathrm{Mg}^{2+}$ und $\mathrm{Cl}>\mathrm{SO}_{4}{ }^{2-}>\mathrm{HCO}_{3}{ }^{-}$. Darüber hinaus spielt auch die weit verbreitete Bewässerung aus dem sulfathaltigen tiefen Grundwasserleiter eine große Rolle. Diese Beobachtung lässt sich durch die relativ hohe Nitratkonzentration in den Brunnen 6 und 14 (Abb. 7-23) erklären.

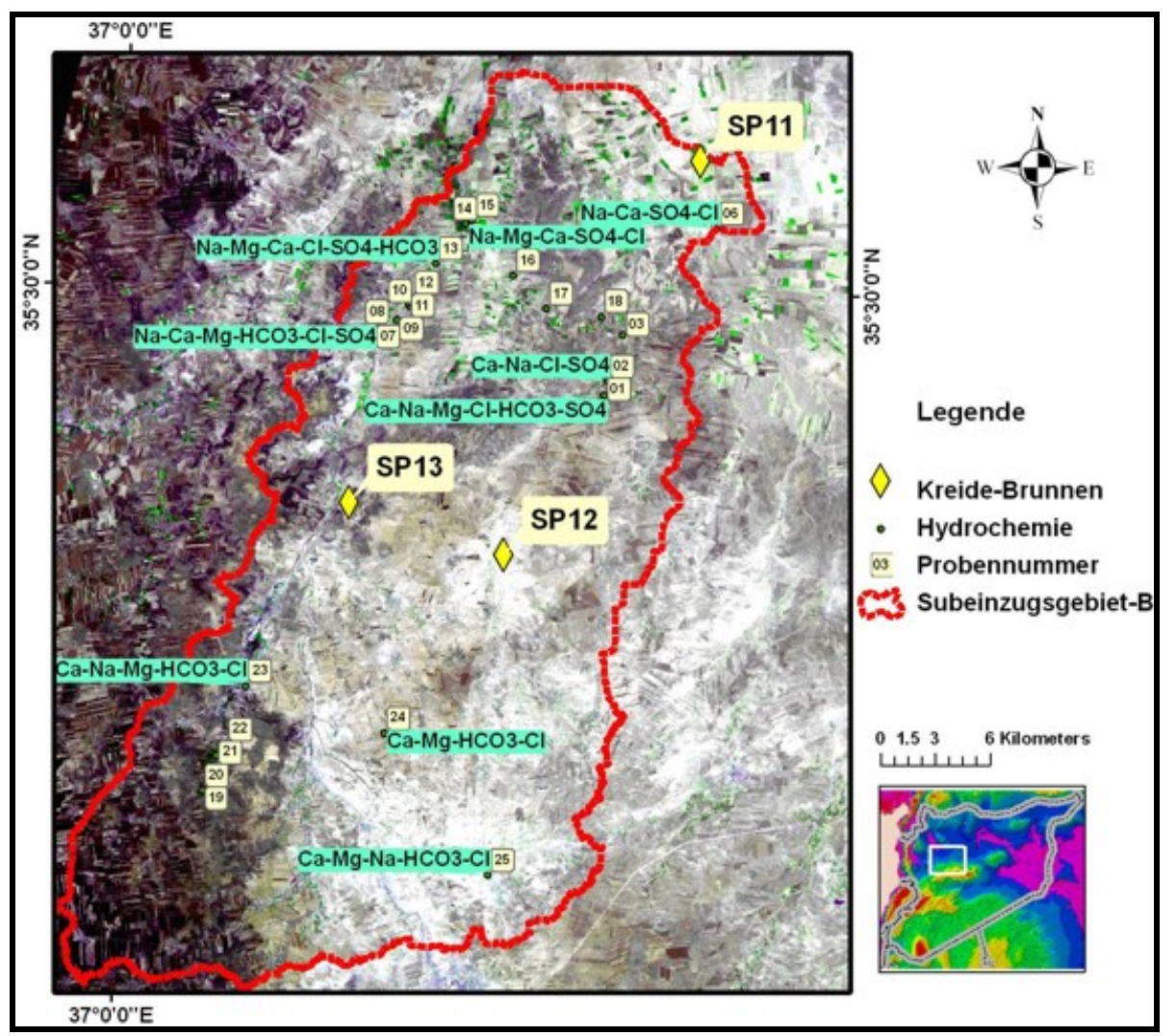

Abb. 7-22: Das Teileinzugsgebiet B 


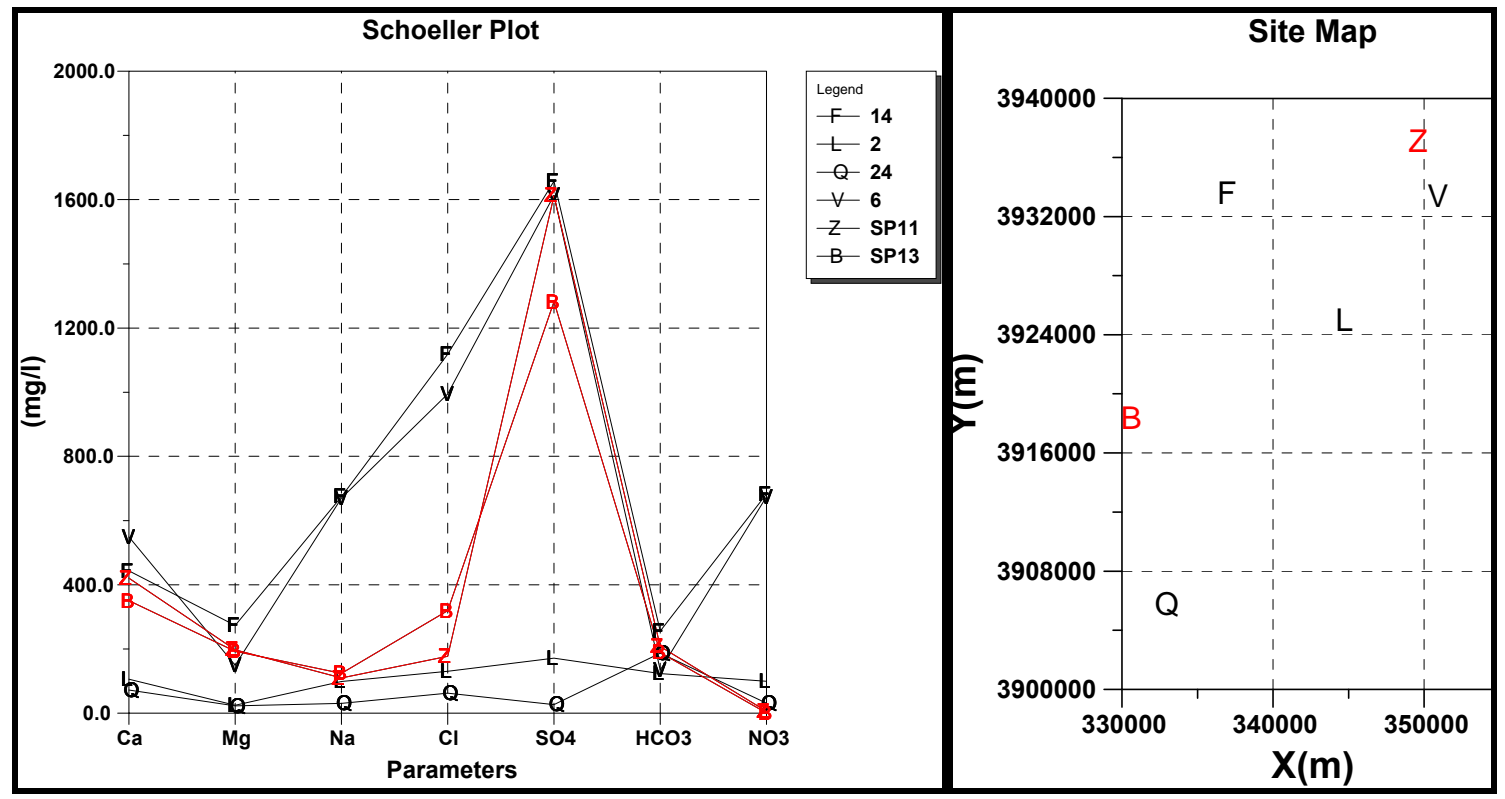

Abb. 7-23: Schöllerdiagramm einiger Paläogen-Brunnen (schwarz) und KreideBrunnen (rot), geographische Positionen (rechts)

\subsubsection{Beobachtung der hydrochemischen Änderung im Winter}

Die Hydrochemie des Regenwassers von 1990 (Tab. 7-8) aus den Wetterstationen Homs und Palmyra, die westlich und südöstlich des betrachteten Gebiets liegen, ergibt durchschnittlich pH-Werte in Höhe von 7,2 bei Homs und 7,4 bei Palmyra. Der Gesamtlösungsinhalt (TDS) im Februar 1990 war bei Homs 93 mg/L und bei Palmyra 115 mg/L (KatTAN, 1997). Der Chemismus des Regenwassers ist deutlich geprägt von Kalziumhydrogenkarbonat, welcher im Allgemeinen auf Grund des Karbonatstaubs in der Atmosphäre entsteht. Diese Ionenkonzentrationen sind verhältnismäßig vergleichbar mit dem Regenwasser der östlichen Mittelmeerregion (RoSENTHAL, 1987). Die relativ hohen Sulfatkonzentrationen in Homs begründen sich durch die industrielle Verunreinigung der Atmosphäre.

Die Feldarbeit fand, wie oben erwähnt, am Ende des Sommers und am Anfang des Winters statt. Allerdings hat sich die Regenzeit 2006 offensichtlich verspätet. Das Regenereignis zwei Tage vor Ende der Feldarbeit erlaubte dennoch die Entnahme von vier Stichproben aus dem Teileinzugsgebiet B. Die Darstellung der chemischen Zusammensetzung im Piperdiagramm (Abb. 7-24) zeigt eine relative Änderung in Richtung eines frischen Grundwassers des Bikarbonattyps (Tab. 7-9). 
Tab. 7-8: Ionenkonzentrationen des Regenwassers in Homs \& Palmyra (Kattan, 1997)

\begin{tabular}{|l|l|l|l|l|l|l|l|l|l|l|l|}
\hline Station & Datum & $\mathbf{p H}$ & $\mathbf{C a}^{2+}$ & $\mathbf{M g}^{2+}$ & $\mathbf{N a}^{+}$ & $\mathbf{K}^{+}$ & $\mathbf{H C O}_{3}{ }^{-}$ & $\mathbf{S O}_{4}{ }^{--}$ & $\mathbf{C l}^{-}$ & $\mathbf{N O}_{3}{ }^{-}$ & $\mathbf{T D S}$ \\
\hline Homs & Jan 90 & 7.95 & 08 & 2.0 & 4.0 & 1.0 & 024 & 13 & 06 & 10 & 68 \\
\hline & Feb90 & 6.24 & 08 & 7.0 & 6.0 & 1.0 & 037 & 14 & 12 & 08 & 93 \\
\hline & Apr90 & 7.30 & 16 & 2.0 & 1.5 & 1.0 & 037 & 12 & 04 & 07 & 81 \\
\hline Palmyra & Feb 90 & 6.98 & 24 & 2.0 & 2.0 & 1.0 & 061 & 09 & 04 & 12 & 115 \\
\hline & Mar 90 & 7.42 & 44 & 5.0 & 2.5 & 1.0 & 110 & 25 & 08 & 10 & 206 \\
\hline
\end{tabular}

Tab. 7-9: Chemischen Zusammensetzung der Brunnen vor und nach dem Regen

\begin{tabular}{|l|l|l|l|l|l|l|l|l|l|}
\hline $\mathbf{N r}$ & \multicolumn{1}{|c|}{$\begin{array}{c}\text { Wasser } \\
\mathbf{T y p e}\end{array}$} & $\begin{array}{c}\mathbf{T D S} \\
\mathbf{m g} / \mathbf{l}\end{array}$ & $\begin{array}{l}\mathbf{N a}^{+} \\
\mathbf{m g} / \mathbf{l}\end{array}$ & $\begin{array}{l}\mathbf{C a}^{2+} \\
\mathbf{m g} / \mathbf{l}\end{array}$ & $\begin{array}{l}\mathbf{M g}^{2+} \\
\mathbf{m g} / \mathbf{l}\end{array}$ & $\begin{array}{c}\mathbf{C l}^{-} \\
\mathbf{m g} / \mathbf{l}\end{array}$ & $\begin{array}{c}\mathbf{S O 4}^{2-} \\
\mathbf{m g} / \mathbf{l}\end{array}$ & $\begin{array}{c}\mathbf{H C O 3}^{-} \\
\mathbf{m g} / \mathbf{l}\end{array}$ & $\begin{array}{l}\mathbf{N O}^{-} \\
\mathbf{m g} / \mathbf{l}\end{array}$ \\
\hline $\mathbf{1}$ & $\begin{array}{l}\text { Ca-Na-Mg-Cl-HCO3- } \\
\text { SO4 }\end{array}$ & 546.2 & 62.8 & 70.7 & 18.7 & 82.0 & 76.5 & 135.8 & 96.6 \\
\hline $\mathbf{1 R}$ & Ca-Na-HCO3-Cl & 731.3 & 70.9 & 104.4 & 22.1 & 83.3 & 78.3 & 269.3 & 99.7 \\
\hline $\mathbf{2}$ & Ca-Na-CI-SO4 & 796.5 & 98.4 & 105.5 & 25.1 & 130.5 & 171.6 & 123.5 & 138.5 \\
\hline $\mathbf{2 R}$ & Ca-Na-HCO3-Cl-SO4 & 996.2 & 109.4 & 141.2 & 28.9 & 138.3 & 179.1 & 250.6 & 145.3 \\
\hline $\mathbf{5}$ & Ca-Na-Mg-CI-SO4 & 787.7 & 94.0 & 105.0 & 27.8 & 157.2 & 124.0 & 134.5 & 142.2 \\
\hline $\mathbf{5 R}$ & Ca-Na-CI-HCO3 & 964.7 & 103.0 & 137.2 & 31.1 & 164.1 & 129.6 & 251.4 & 145.1 \\
\hline
\end{tabular}

Die Schöllerdiagramme der Proben 1, 2 und 5 vor und nach dem Regenereignis zeigen eine Erhöhung der Bikarbonat- und Kalziumkonzentration (Abb. 7-25). Diese hängt höchstwahrscheinlich von zwei Faktoren ab. Der erste Faktor ist die direkte Infiltration des Regenwassers und der zweite Faktor ist die Strömung des alten Grundwassers aus den benachbarten Gebieten unter dem Druck des neu gebildeten Grundwassers. 


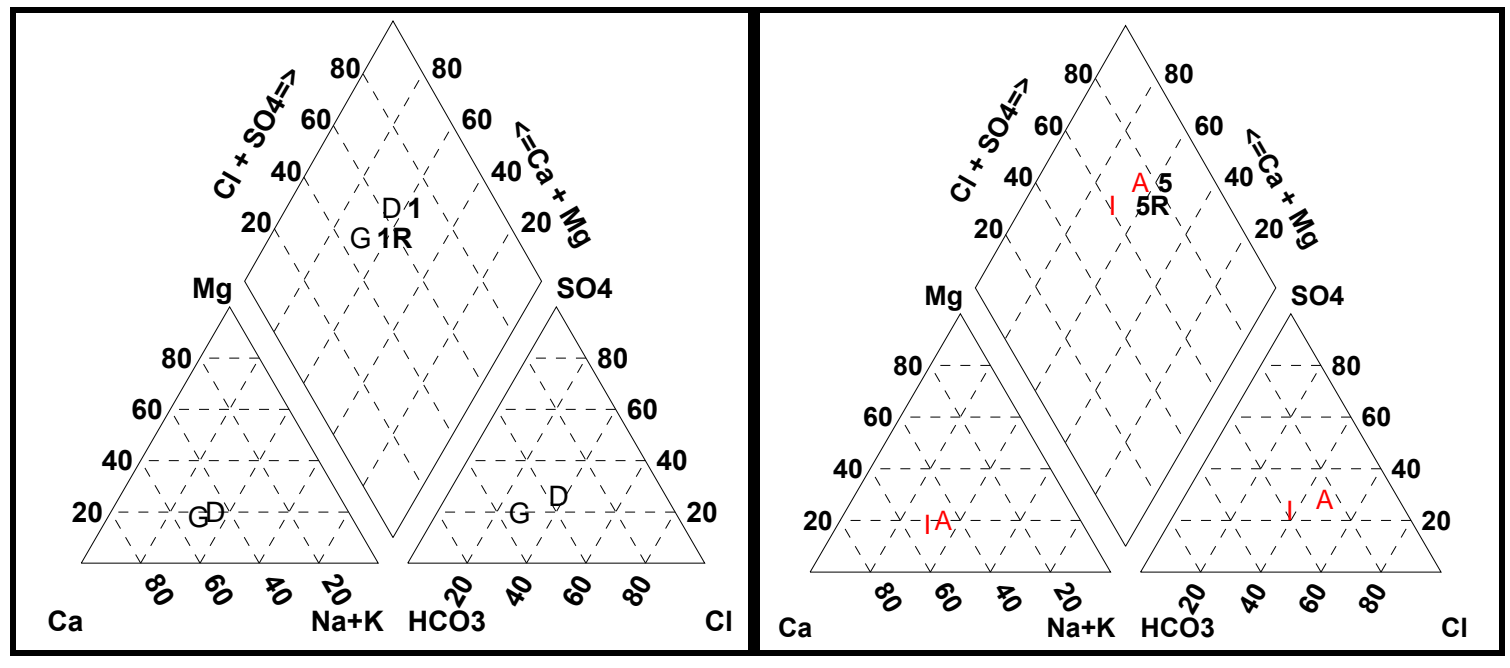

Abb. 7-24: Piper-Diagramm der Proben vor Regenereignis (Nr.) und danach (Nr. R)
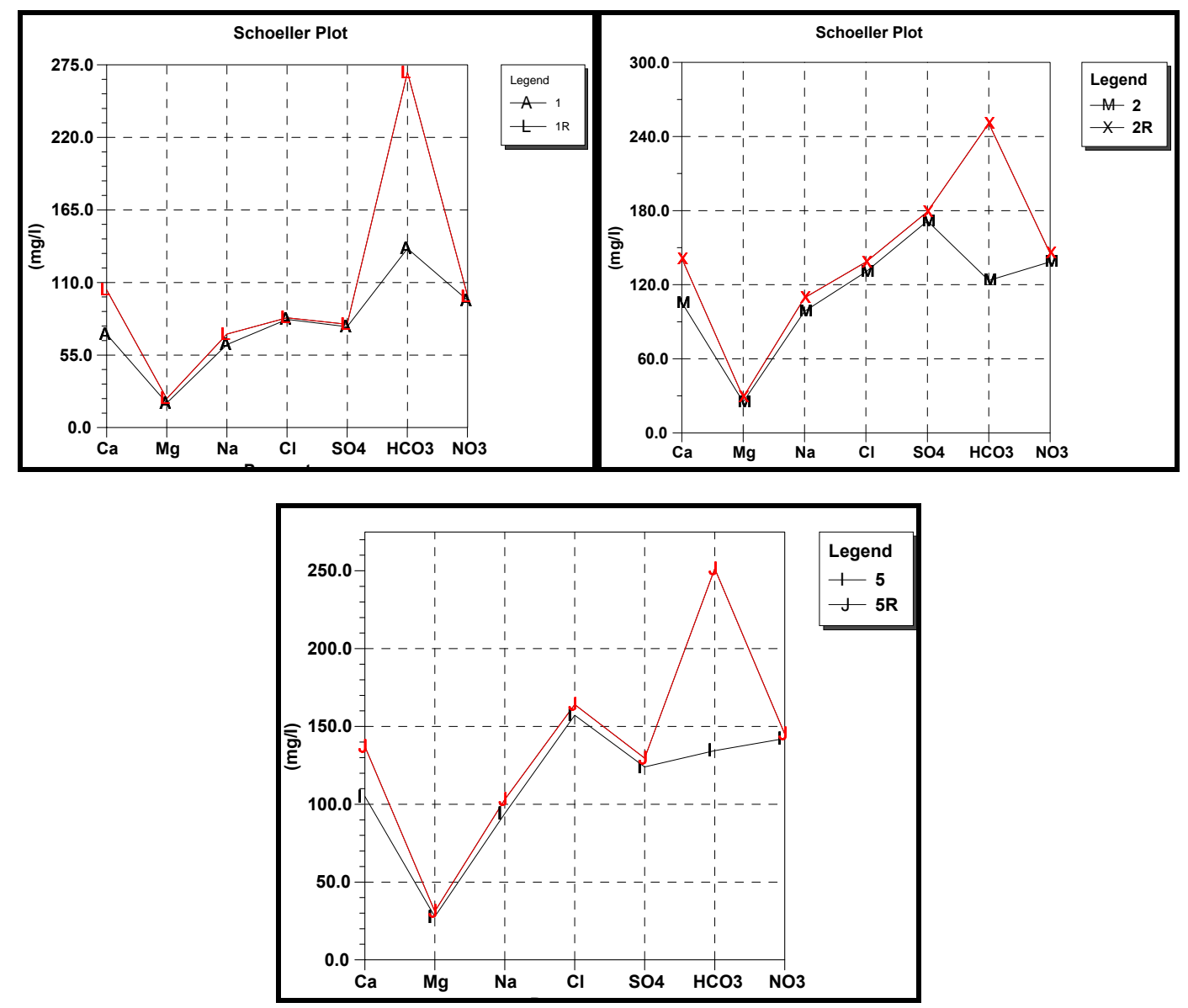

Abb. 7-25: Schöllerdiagramm der Proben vor dem Regenereignis und danach 


\section{Ka p it e l 8}

\section{Zu s a m m e n f s s u n g}




\section{Zusammenfassung}

Im Rahmen der Arbeit wurden verschiedene geomorphologische Methoden kombiniert. Dabei wurde ein breites Spektrum verfügbarer Daten (Höhendaten, Satellitenbilder und Kartenmaterialien) sowie Geländedaten unter Anwendung bewährter Methoden und neu entwickelter Ansätze ausgewertet und ihre Einsatzmöglichkeiten in Bezug auf die angewandte Geomorphologie an Beispielen im Nordwesten Syriens untersucht. Dabei wurde eine umfangreiche Palette von Werkzeugen zur Visualisierung, Ableitung, Verwaltung, Analyse und Erweiterung raumbezogener Daten verwendet. Die Arbeit zielte darauf ab, einen Beitrag zum besseren Verständnis der geomorphologischen und hydrologischen/hydrogeologischen Gegebenheiten des Untersuchungsgebiets zu leisten. Ein weiterer Schwerpunkt im Bereich der angewandten Geomorphologie wurde auf die hydrologischen/hydrogeologischen Aspekte gelegt, da im Nordwesten Syriens das Wassermanagement ein Hauptfaktor für eine nachhaltige Entwicklung ist.

\subsection{Die Resultate}

Entsprechend der in Kapital 1.1 formulierten Zielsetzungen ist dieses Kapital thematisch dreigeteilt. In dem ersten Teil der Arbeit wurden durch Einsatz des digitalen Höhenmodells und durch GIS-gestützte Verfahren die geomorphologischen Einheiten in einem Areal von mehreren tausend $\mathrm{km}^{2}$ klassifiziert. Auf Basis der gegenseitigen Beziehung zwischen zwei geomorphometrischen Größen (die absolute Höhe und die Hangneigung) konnte die regionale Klassifikation der morphologischen Einheiten ermittelt werden. Die Form dieser Einheiten konnte mit Hilfe von GIS-Funktionalitäten geomorphometrisch morphologisch vollständig beschrieben werden. Weiterhin wurden mehrere Satellitenbilder des Untersuchungsgebiets (Landsat \& ASTER) ausgewertet, wodurch viele Aussagen über die Geomorphologie, vor allem über die oberflächlichen Sedimente bzw. Lithologie, erlangt werden konnten. Dabei wurden vielfältige Fernerkundungsverfahren eingesetzt, um möglichst viele geomorphologische/geologische Informationen aus den Satellitenbildern abzuleiten. Die Eignung bestimmter Bilder und Verfahren für die geomorphologischen Untersuchungen in dem betrachteten Gebiet konnte dadurch überprüft werden. Die Verfahren der Falschfarbendarstellung, der RatioBildung und der Hauptkomponentenanalyse (PCA) eigneten sich um die lithologischen geomorphologischen Einheiten gut zu erfassen. Einige Verfahren, wie die Kantenstärkung, haben sowohl bei der Auswertung der einzelnen Bänder als auch bei der Falschfarbendarstellung sowie bei der Kartierung der Lineamente gute Ergebnisse erzielt. 
Da das Ziel des ersten Teils nicht die detaillierte Kartierung der Landoberfläche des ganzen Areals, sondern eine geomorphologische Regionalisierung ist, wurde vielmehr die Nutzbarkeit der verschiedenen Daten für die geomorphologische Regionalisierung mittels der Fernerkundungs- und GIS-Verfahren untersucht. Daher war die Bearbeitung sowohl der Höhendaten als auch der Satellitenbilder auf die visuelle und numerische regionale Darstellung ausgerichtet, wodurch ein realitätsnaher geomorphologischer Status dargestellt wurde.

Es wurde gezeigt,

- wie Geographische Informationssysteme (GIS) schnelle, präzise und reproduzierbare Berechnungen der geomorphometrischen Parameter aus digitalen Höhenmodellen (DHM) erlauben.

- wie das gut vorbereitete, optimierte digitale Höhenmodell (SRTM) mit Hilfe von GISFunktionalitäten sowohl die qualitative als auch die quantitative geomorphologische Georeliefbeschreibung eines Gebiets liefern kann.

- wie das digitale Höhenmodell bzw. die Überlagerung der Satellitenbilder über das DHM als wertvolles Hilfsmittel am Rechner eingesetzt werden kann, um komplexe als auch einfache geomorphologische/geologische Flächen und Strukturen zu visualisieren, wohingegen solche Visualisierungen bei stereoskopischen Beobachtungen zeitlich viel aufwendiger sind.

- und schließlich wie das digitale Höhenmodell von SRTM durch seine regionale Abdeckung, die Extraktion hydrologischer Merkmale (Entwässerungsnetz, Wasserscheide bzw. Einzugsgebiete) ermöglicht, die für die Untersuchung des Verhältnisses zwischen Geomorphologie und Geologie in einem ausgedehnten Gebiet von entscheidender Bedeutung sind.

Im zweiten Teil der Arbeit wurde eine morphostrukturelle Analyse durch Einsatz des digitalen Höhenmodells und der Satellitenbilder durchgeführt. Die Analyse erfolgte durch die analoge und digitale Ermittlung der geomorphologischen und morphostrukturellen Merkmale. Mit der Anwendung von GIS-Funktionalitäten wurde zuerst die gegenseitige Beziehung zwischen zwei geomorphometrischen Größen, nämlich der Hangneigung und der Exposition, in Verbindung mit den Richtungen der Morphostrukturen diskutiert. Im darauf folgenden Schritt wurden die linearen Morphostrukturen auf Basis des geschummerten Reliefmodells und der Satellitenbilder interpretiert. Für die Auswertung der linearen Morphostrukturen auf Basis des geschummerten Reliefmodells wurde das digitale Höhenmodell von SRTM zusätzlich ver- 
wendet. Hierbei waren für die Festlegung der Beleuchtungsazimute die Vorkenntnisse der dominierenden Richtungen der Morphostrukturen von großer Bedeutung. Ebenfalls spielten die Funktionen der vertikalen Erhöhung und der möglichen Änderung der Azimut- sowie Sonnenhöhen-Werte eine große Rolle. Die GIS-Funktionalitäten waren nicht nur bei der Aufbereitung und der Auswertung der Daten außerordentlich nützlich, sondern auch bei der Überprüfung der Ergebnisse. Von großem Vorteil ist besonders die Möglichkeit, vorhandene und erzeugte Daten zu überlagern. Durch die Überlagerung konnte gezeigt werden, dass einige verborgene Großstrukturen im betrachteten Gebiet, wie die Störungen im Vorland der Palmyra-Ketten, durch klare geomorphologische Ausprägungen (Umbiegung oder Verschiebung von Wadis) auf Satellitenbildern erkennbar sind.

Im letzten Teil der Arbeit wurden die geomorphologischen Informationen bzw. Aussagen, die im Lauf der Arbeit gewonnen wurden, zweckgerichtet integriert. Allgemein wurde hier der praktische Ansatz der Geomorphologie behandelt. Dieser Ansatz der geomorphologischen Informationen wurde durch die Integration von mehreren Landschaftselementen in einem thematisch-sachlichen Kontext am Beispiel des südöstlichen Bereichs des Untersuchungsgebiets durchgeführt. Kern dieses Arbeitsschritts war eine mehrfach-gestufte Kennzeichnung Raster-basierter Analyse. Durch die Nutzung der GIS-Funktionen konnten numerische Abfragen nach hydrogeomorphologischen Gesichtspunkten entwickelt werden. In diesem Zusammenhang konnten die geoökologischen Funktionen des Georeliefs mit anderen abschätzbaren Randbedingungen des geoökologischen Systems, die den Zustand des Grund- und Oberflächen-Wassers im Untersuchungsgebiet mehr oder weniger stark beeinflussen, zum Einsatz gebracht werden. In diesem Sinne konnte eine detaillierte Kennzeichnung der Geomorphologie durch die zusätzlichen Informationsquellen (Satellitenbilder von ASTER und Feldarbeit mit GPS-Messungen) erreicht werden. Die Beschreibung der geomorphologischen Einheiten konnte dadurch verfeinert und die Grenzen zwischen ihnen konnten genau definiert werden. Die Verwendung von GPS-Messungen war sehr wichtig für die Verknüpfung der vorhandenen Daten im ArcGIS mit den aus Feldarbeit gewonnen Daten sowie für die Nachbearbeitung.

Bei der hydrogeomorphologischen Bewertung wurden mehrere geomorphologische Aspekte (geomorphologischen Einheiten, Hangneigung, Entwässerungsnetzdichte und Lineamente) betrachtet, welche die besondere Rolle der Geomorphologie im betrachteten Gebiet hervorheben. 
Das Resultat war eine hydrogeomorphologische Darstellung, die Potentialzonen für die Grundwasserneubildung enthält. Im Laufe dieses Arbeitsschritts hat sich die Kombination der Fernerkundungsdaten mit dem digitalen Höhenmodell als sehr nützlich erwiesen. Eine weitergehende Einzugsgebietsuntersuchung war erst durch die Einbeziehung von aktuellen Satellitendaten möglich, um die Umrisse der unter anthropogenen Aktivitäten umgewandelten Teileinzugsgebiete zu ermitteln. Dadurch konnten zwei kleine Teileinzugsgebiete definiert werden, die Bereiche mit diversen geomorphologischen Merkmalen und verschiedenen Potentialen für Grundwasserneubildung umfassen. Dies war sehr wichtig für die Planung der nachfolgenden Geländearbeit.

Das Ergebnis der Potentialzonen für die Grundwasserneubildung konnte mittels der chemischen Analyse des Grundwassers aus den beiden ausgewählten Teileinzugsgebieten bewertet und die Wechselwirkung zwischen den chemischen Materialeigenschaften der Landoberfläche und dem Grundwasser beobachtet werden. Bei der Betrachtung der chemischen Analyse wurde festgestellt, dass der synthetischen Karte ergänzend zu den vorhandenen hydrogeologischen Karten eine erhebliche Bedeutung für den Informationsgewinn zukommt. Auf diese Weise wurden die physikalischen und chemischen Eigenschaften der Verwitterungsausgangsmaterialen, die sehr stark in das Interessenfeld der Geomorphologie rückten, ausgedrückt. Hierbei zeigte sich der Einfluss der Verwitterung der verschiedenen lithologischen Einheiten (Basalt, Kreide bzw. Kalkstein etc.) und der Produkte der geomorphologischen Prozesse (Versalzung).

Zusammenfassend hat diese Studie zur Entwicklung einer umfassenden Datenbank geführt, welche geomorphologische/geologische und geoökologische Faktoren integriert. Mithin wurde die Tauglichkeit von GIS-Verfahren für die hydrogeomorphologische Problemstellung untersucht. 


\subsection{Diskussion}

Die geomorphologische Klassifikation der Haupteinheiten verfeinerte sich mit der Entwicklung der EDV. In Anlehnung an die Einteilung in physiographische Einheiten von FENNEMAN (1931) erfolgten mehrere wissenschaftliche Arbeiten wie die Arbeiten von MiLIARESIS \& Argialas (1999) und Miliaresis (2001). Solche Arbeiten beschäftigten sich mit Georeliefklassifikation auf einem sehr klein Maßstab in Regionen mit deutlich gebirgigem Charakter (z. B. Zagros in Iran). In der vorliegenden Arbeit war die Entwicklung einer eigenen Klassifikation erforderlich, da die meisten Teile des Untersuchungsgebiets flach sind. Dabei wurden die Bearbeitungsmöglichkeiten der geomorphometrischen Kriterien in ArcGIS, wie Verschneidung, Klassifikation, Kalkulation, Filterung und Konvertierung von Rasterdaten zu Vektordaten und umgekehrt, verwendet. Gegenüber anderen Arbeiten, die sich mit Georeliefklassifikation beschäftigen, hat diese Arbeit eine neue Ausrichtung der geomorphologischen Darstellung (FLORINSKY, 1998), nämlich die Kombination der digitalen Höhendaten mit Satellitenbildern in GIS, verfolgt. Hinzu kamen auch die Informationen aus Kartenmaterialien. Ansonsten konnte die Darstellung und Beschreibung der Geomorphologie auf Basis des digitalen Höhenmodells allein nicht alle wesentlichen Informationen für eine geomorphologische Detailanalyse liefern.

Bei der Auswertung der Satellitenbilder wurden Verfahren der Ratio-Bildung und der Hauptkomponentenanalyse (PCA) stärker eingesetzt, weil Sie gegenüber anderen Verfahren der Fernerkundung die Möglichkeit bieten, die geomorphologischen Erscheinungen stärker zu visualisieren und auswerten zu können. Das Prinzip der Hauptkomponentenanalyse (PCA) erzielt, sowohl durch die RGB-Kombination der produzierten PC-Bänder als auch durch die Rücktransformation der Bänder, gute Ergebnisse.

Der flache Charakter des Georeliefs war der bestimmende Faktor dafür, dass bei der Auswertung der linearen Morphostrukturen das digitale Höhenmodell (SRTM) gewählt wurde, obwohl die Erstellung eines Höhenmodells aus den vorhandenen topographischen Karten möglich war. Der Grund ist, dass bei Erstellung eines Höhenmodells aus den topographischen Karten mehrere Höheninformationen verloren gehen können, nämlich bei der Interpolation der weit voneinander entfernten Höhenlinien in den flachen Bereichen. Hingegen wurde bei dem digitalen Höhenmodell von SRTM die Höhe für jede Zelle erzeugt. In diesem Fall ist die Gefahr, die tektonischen Strukturen mit kleinen morphologischen Merkmalen zu verlieren, viel geringer als im Fall der topographischen Daten. Deshalb ist das Höhenmodell von SRTM 
im Vergleich zu den Modellen, die aus den topographischen Karten erzeugt werden, viel besser für die Auswertung der linearen Morphostrukturen geeignet. Darüber hinaus hat die Kombination zwischen zwei Informationsquellen (SRTM und Satellitenbilder) zur Folge, dass die Genauigkeit der geographischen Lage der ausgewerteten Lineamente und die Wahrscheinlichkeit, dass die Lineamente in der Tat einen geomorphologischen/geologischen Ursprung haben, erhöht werden. Beispielsweise hat die Verfolgung der Lineamente auf geschummerten Reliefmodellen der SRTM (ein Pixel ist 90m) in Bezug auf die geographische Position einen Mangel wegen der seitlichen Verschiebung gemäß der Beleuchtung. Obwohl die Verschiebung einiger Pixel (ein Pixel ist hier 90m) im regionalen Maßstab nur wenig Einfluss hatte, war der Vergleich der Lineamente mit den Satellitenbildern (ein Pixel entspricht hier 30m) sehr hilfreich. Andererseits hat die Anwendung von Satellitenbildern allein Nachteile, da einige Phänomene wie Wege und Landnutzung in den Satellitenbildern manchmal als Lineamente (künstliche Lineamente) ausgewertet werden können, welche in dem digitalen Höhenmodell nicht auftreten.

In der vorliegenden Arbeit wurden im Vergleich zu anderen Arbeiten Vorortuntersuchungen mittel GPS Messungen durchgeführt. Dadurch wurde festgestellt, dass in vielen Bereichen der ausgewerteten Lineamente geklüftete und/oder verkarstete Gesteine vorhanden sind. Diese Beobachtungen und die gute Übereinstimmung der als Richtungsrose dargestellten Richtung der linearen Morphostrukturen mit den Literaturbefunden lassen vermuten, dass derartig ausgewertete lineare Morphostrukturen oberflächliche oder unterirdische geologische Strukturen wiedergeben. Folglich konnte angenommen werden, dass die morphologischen Lineamente (Kanten, Hänge, Wadis und Schichtstufen) in dem betrachteten Gebiet stark an tektonische Strukturen gebunden sind. Der Einsatz von GPS-Messungen in dieser Arbeit hat auch andere Vorteile im Vergleich zu den traditionellen Feldarbeitmethoden. Durch die Vorortaufnahmen bietet jeder gemessene Punkt eine direkte Anbindung zur Realität, was Ergebnisse mit besserer Genauigkeit und Ausführlichkeit erbracht hat. Während viele wissenschaftliche Arbeiten sich nur wie im ersten Teil dieser Arbeit mit dem Georeliefzustand (geomorphographischer/geomorphometrischer Ansatz), oder wie im zweiten Teil nur mit der morphostrukturellen Analyse, beschäftigt haben, hat diese Arbeit einen praktischen Ansatz entwickelt. Dabei wurde die Geomorphologie als ein komplexes System mit einer Vielzahl von Wechselwirkungen zwischen endogenen und exogenen Prozessen berücksichtigt. Hierbei wurden die geoökologischen Georelieffunktionen verwendet, um die hydrologischen/hydrogeologischen Fragestellungen zu lösen. 


\subsection{Ausblicke}

Über den Rahmen dieser Arbeit hinaus legen die hier angewandten Methoden und vorliegenden Ergebnisse eine Reihe weiterführender Untersuchungen nahe. Vor allem durch Einbindung neuer Fernerkundungsdaten mit höherer geometrischer Auflösung und höherer oder anderer Spektraleigenschaften (Radardaten) ist weitere Information $\mathrm{zu}$ gewinnen. Die Einbeziehung weiterer multitemporaler Satellitenbildern in die erstellte Datenbank ermöglicht bessere Beobachtung bzw. Analyse der feuchten Bereiche. Die Radardaten sind weiterhin von hohem Informationsgehalt. Sie können, einerseits die Untersuchung der Bodenfeuchte ermöglichen, und andererseits der Erkundung der geologischen Strukturen, sowohl die aufgeschlossenen Strukturen als auch die unter den lockeren Materialien begrabenen, dienen. Diese Daten können mit anderen Daten dieser Studie gekoppelt werden, wodurch die geomorphologische morphostrukturelle Auswertung und Analyse erweitert und verifiziert werden kann. Weiterhin kann die durchgeführte morphostrukturelle Analyse als Basis für die Planung geophysikalischer Messungen dienen. Die Einbeziehung solcher Daten (geophysikalischer Daten) in ArcGIS ist prinzipiell möglich und empfehlenswert, wenn eine verifizierte strukturelle Identifikation das Ziel ist.

Bezüglich der Erstellung einer hydrogeomorphologischen Datenbank kann eine weitere Ergänzung bzw. Vertiefung durch die Kennzeichnung der Feuchtbereiche geschaffen werden. Das erfordert die Anwendung von digitalen Höhendaten mit höherer Auflösung. Dadurch können die topographischen Senken in dem Gebiet extrahiert werden, welche als mögliche natürliche Wassersammler dienen bzw. feuchte Bereiche abbilden können. Selbstverständlich kann eine absolute Kennzeichnung der Feuchtbereiche aus den digitalen Höhenmodellen allein nicht ermittelt werden. Diesbezüglich bieten multitemporale Satellitenbilder oder Radardaten eine gute Ergänzung.

Auf Grund dessen, dass dieses Gebiet hydrologisch nicht instrumentiert ist (keine hydrologische Messungen des Oberflächenwasserabflusses), bietet die Analyse der Beziehung zwischen der Geomorphologie und der Hydrochemie viele Möglichkeiten für das Wassermanagement und -monitoring. Die chemischen Analysen sind nützlich für die Identifizierung der Grundwasserneubildung und können ausgeweitert werden, indem weitere ereignisorientierte Grundwasserproben genommen werden. Weiterhin kann diese Darstellung für das ganze Untersuchungsgebiet verallgemeinert werden. Folglich kann die erfolgreiche Einführung und Anwendung der hydrogeomorphologischen Datenbank die Bildung einer Informationsinfra- 
struktur ermöglichen, wobei weitere Daten (z. B. hydrologische Messungen bzw. Beobachtungen, Bewässerungsverbrauch, geophysikalische Messdaten), die einen entscheidenden Beitrag für die Entwicklung Syriens leisten können, einzubeziehen sind.

Im Südosten des Untersuchungsgebiets kann die Existenz der tektonisch bedingten linearen Wadis eine sehr gute Bedingung für Grundwassergewinnung sein. In den Bereichen solcher Wadis sollten Methoden des Water Harvesting angebracht werden, um die Verdunstungsverluste zu verringern. In den inneren Bereichen des betrachteten Gebiets ist, zum einen die Wasserinfiltration sehr gering, und zum anderen das infiltrierte Wasser zur Versalzung des Grundwassers führt. Im Norden, wo große Teile der Bevölkerung Syriens leben und eine verbreitete Landnutzung stattfindet, sollte diese Darstellung durch verstärkte Berücksichtigung der anthropogenen Einflüsse auf die Hydrochemie des Oberflächenwassers, der Böden und des Grundwassers erfolgen.

Die Beobachtungen dieser Arbeit bezüglich der Interaktion zwischen der Geomorphologie und dem Grundwasser zeigen, dass die Beseitigung der Kalkkrusten bzw. der Anbau von Olivenbäumen eine sehr empfehlenswerte Idee ist, welche von den Planern und der Regierung unterstützt werden sollte.

Die Schilderung dieses Vorhabens zeigt, wie das explosive Wachstum der rechnergestützten Kapazitäten neue Ansätze der Geomorphologie entstehen lässt, und wie das geographische Informationssystem (GIS) Disziplinen der Geomorphologie, der Hydrologie, der Geologie, der Pedologie und der Fernerkundung sehr eng verbunden hat. Die fortschreitende Entwicklung der Möglichkeiten der Integration der vielfältigen Daten der genannten Disziplinen in Form von Datenbanken verstärkt die Annahme, dass diese Kopplung weiterhin wachsen wird. Gleichzeitig stellen die traditionellen Techniken wie die Feldkartierung mit Unterstützung der GPS-Messungen wichtige Bodendaten zur Verfügung. Dementsprechend ist anhand der ausführbaren größeren Präzision über größere räumliche Skalen zu vernehmen, dass der dynamische und interdisziplinäre Aspekt der Geomorphologie mehr direkt durch diese Entwicklungen in den Vordergrund tritt.

Schließlich wird durch diese Arbeit die Tatsache bekräftigt, dass die Dominanz der Fernerkundungs- und GIS-Methoden die Betrachtung der Geomorphologie aus anderen Blickwinkeln zur Folge hat. Andererseits wurde dabei argumentiert, wie der zunehmende Gebrauch von Satellitenbildern kombinierend mit dem digitalen Geländemodellen die Geomorphologie in einem stark angewandten Wissenschaft wandelt, womit sie auf mehrere Fragestellungen der 
Umweltforschung reagieren kann. Durch seine kartographischen Techniken und durch sein Vermögen, die gewonnenen Informationen und die Resultate der geomorphologischen Analysen höchst deskriptiv und quantitativ darzustellen, stellt sich das GIS als eine Einheit der geomorphologischen Analysen dar.

Aufbauend auf den hier gewonnen Erkenntnissen könnten in zukünftigen Studien GISbasierte hydrogeomorphologische Datenbanken auf andere Gebiete übertragen werden, besonders in Gebieten, in denen wenige hydrologische Beobachtungen bzw. Messungen vorhanden sind oder geplant werden sollen. Die hier vorgestellte Methode stellt eine mögliche Grundlage für diesbezüglich weiterführende Untersuchungen dar. 
- 156 - 


\section{Literaturverzeichnis}

Aber, J. S.; Spellman, E.E. \& Webster, M. P. (1993): Landsat remote sensing of glacial terrain, in Aber, J. S.: Glaciotectonics and Mapping Glacial Deposits, Proceedings of the INQUA Commission on Formation and Properties of Glacial Deposits, S. 215-225.

AKMAN A. Ü. \& TÜFEKÇI K. (2004): Determination and characterisation of fault systems and geomorphological features by RS and GIS techniques in the WSW part of Turkey. XXth ISPRS congress, Istanbul, S. 899-904.

AlberTZ, J. (2001): Grundlagen der Interpretation von Luft- und Satellitenbildern. Eine Einführung in die Fernerkundung. 2. Auflage, 250 Seiten, Darmstadt (Wissenschaftliche Buchgesellschaft).

Al-Juaidi, F.; Millington, A. C. \& McLaren, S. J. (2003): Merged remotely sensed data for geomorphological investigations in deserts: examples from central Saudi Arabia. The Geographical Journal, V. 169, Nr. 2, S. 117-130.

Aller, L.; BenNett, T.; LeHR, J.H. \& Petty, R.J. (1985): A Standard System for Evaluating Ground Water Pollution Potential Using Hydrogeologic Settings. EPA/600/2-85/018, R.S. Kerr Environmental Research Laboratory, U.S. Environmental Protection Agency, Ada, Oklahoma, 621 Seiten.

BÄHR, H. P. \& VöGTLE, T. (1991): Digitale Bildverarbeitung; Anwendungen in Photogrammetrie und Fernerkundung. S. 417, Karlsruhe (Wichmann).

BAmler, R. \& HARTL, P. (1998): Synthetic Aperture Radar Interferometry. - Inverse Problems, V. 14, S. 1-54.

BARSCH, D. \& CAINE, N. (1984): The nature of mountain geomorphology, Mountain Research and Development, V. 4, Nr. 4, S. 287-298.

BERGER, Z. (1994): Satellite hydrocarbon exploration. Interpretation and integration techniques. Springer Verlag.

BEsLER, H. (1992): Geomorphologie der ariden Gebiete, Darmstadt (Wissenschaftliche Buchgesellschaft). 
BLAIR, R.W. (1986): Regional landform analysis United States (NASA SP-486). In: Short, N.M., Blair, R.W. (Eds.), Geomorphology from Space, a Global Overview of Regional Landforms. Government Printing Office, Washington, DC, S. 1-22.

BLAIR, R. W. (2003): Geomorphology: Boundaries between Media, Concepts and Modelling in Geomorphology: International Perspectives, Eds. I. S. Evans, R. Dikau, E. Tokunaga, H. Ohmori and M. Hirano, S. 33-42. TERRAPUB, Tokyo, 2003.

Bösch, H. (1949): Beiträge zur Morphologie des Nahen Osten. Eclogae geologicae Helvetiae 42, S. 23-33.

Bottema, S. \& BARKOUdAH, Y. (1979): Modern pollen precipitation in Syria and Lebanon and its relation to vegetation, Pollen et Spores, V. 28, S. 427 - 480.

Brahana, J. V.; Thrailkill, J.; Freeman, T. \& WARD, W. C. (1988): Comparative hydrogeology of carbonate rocks, in Back, W., Seaber, P. R., and Rosenshein, J. S., Ground Water Hydrogeology, Boulder, Colorado, Geol. Society of America, V. 0-2, S. 333-352.

BRASSEL, K. (1974): A model for automatic hill-shading: American Cartographer, v. 1, no. 1, S. $15-27$.

Brew, G.; Barazangi, M.; Al-Maleh, A. K. \& SAwaF, T. (2001): Tectonic and Geologic Evolution of Syria. Geo Arabia, V. 6, Nr. 4, 573-616.

Burbank, D.W. \& Anderson, R.S. (2001): Tectonic Geomorphology. Blackwell Science, London, $274 \mathrm{~S}$.

BuRDON, D. J. (1961): Report to the Government of the United Arab Republic (Syrian Region) on Groundwater Development and Conservation in Syria. Vervielf. Manuskr. FAO, Rom. (ETAP-Report Nr. 1270).

Byrd, J.O.D.; Smith, R.B. \& GeISSMan, J.W. (1994): The Teton fault, Wyoming: neotectonics, and mechanisms of deformation. J. Geophys. Res. 99, B10, S. $20095-20122$.

CARroll, D. (1962): Rainwater as a chemical agent of geologic processes: A review. U.S. Geol. Survey Water-Supply Paper 1535-G, S. 18-20.

Chaimov, T.; Barazangi, M.; Al-SaAd, D., Sawaf, T. \& Gebran, A. (1990): Crustal shortening in the Palmyride fold belt, Syria, and implications for movement along the Dead Sea fault system. Tectonics, V. 9, S. 1369 - 1386. 
Chavez, P.S.; BerLin, G.L. \& Sowers, L.B. (1982): Statistical method for selecting Landsat MSS ratios. J. of Appl. Photographic Eng. V. 8, S. 23-30.

Chavez, P. S. (1989): Radiometric calibration of Landsat Thematic Mapper multispectral images, Photogrammetric Engineering and Remote Sensing, V. 55, Nr. 9, S. 1285-1294.

Chavez, P. S. (1991): Comparison of three different methods to merge multiresolution and multispectral data: Landsat TM and SPOT Panchromatic. Photogrammetric Engineering and Remote Sensing, V. 57, S. 295-303.

Chorley, R. J. (1969): The drainage basin as the fundamental geomorphic unit. In: Water, Earth and Man, Kapital 2, S. 77-99, Methuen, London.

Chorowicz, J.; Dhont, D. \& GÜNDOĞDU, N. (1999): Neotectonics in the eastern North Anatolian fault region (Turkey) advocates crustal extension: mapping from SAR ERS imagery and Digital Elevation Model. Journal of structural Geology, V. 21, S. 511-532.

Clark, C.D. \& Wilson, C., (1994): Spatial analysis of lineaments. Computur Geoscience, V. 20, S. 1237-1258.

Collet, B.; Taud, H.; Parrot, J.F.; Bonavia, F. \& Chorowicz, J. (2000): A new kinematic approach for the Danakil block using a Digital Elevation Model representation. Tectonophysics, V. 316, S. 343-357.

DEMEK, J. (1976): Handbuch der geomorphologischen Detailkartierung, Wien.

DemeK, J.; EMBLeton \& C., Kugler, H. (1982): Geomorphologische Kartierung in mittleren Maßstäben. VEB Hermann Hack, Gotha.

DIKAU, R. (1988): Entwurf einer geomorphographisch-analytischen Systematik von Reliefeinheiten, Heidelberger Geographische Bausteine, V. 5, S. 1-45, Heidelberg.

DLR, (02.12.2007): Zitieren aus dem World Wide Web. URL: http://www.dlr.de/srtm/level1/howto_ge.htm.

DRURY, S.A. (1998): Images of the earth - a guide to remote sensing, 2. Auflage, S. 203, Oxford (Oxford University Press).

DRURY, S.A. (2001): Image interpretation in Geology. Auflage 3, Nelson Thornes, Cheltenham, UK. 
Dubertret, L. \& Vautrin, H. (1938): Sur l'existence du Pontien lacustre en Syrie et sur sa signification tectonique. Comptes Rendus Hebdomadaires des Séances de l'Academie des Sciences de Paris, V. 206, S. 69-71.

DwIVEDI, R.S. \& RAO, B.R.M. (1992): The selection of the best possible Landsat TM band combination for delineating salt-affected soils. Int. J. R.S., V. 13, Nr. 11, S. 2051-2058.

EvAns, I. S. (1972): General Geomorphometry, derivatives of altitude and descriptive statistics, In: Spatial Analysis in Geomorphology (Ed, Chorley, R. J.) Harper and Row, New York, S. 17-90.

EvANS, I.S. (1980): An integrated system of terrain analysis and slope mapping. Zeitschrift fur Geomorphologie, Suppl. Bd. 36, S.274-295.

Fenneman, N. (1931): Physiography of Western United States. McGraw-Hill, New York, S. 534.

FLORINSKY, I.V. (1998): Combined analysis of digital terrain models and remotely sensed data in landscape investigations, Progress in Physical Geography, V. 22, S. 33-60.

FRISCH, W. (1997): Tectonic Geomorphology. In Proceeding of the Fourth Int'l. Conf. on Geomorphology, Z. Geomorphologie N. F., Suppl. Bd. 118.

Ganas, A.; Papadopoulos, G. \& PAvlides, S.B. (2001): The $7^{\text {th }}$ September 1999 Athens 5.9 Ms earthquake: remote sensing and digital elevation model inputs towards identifying the seismic fault. International Journal of Remote Sensing 22, 191- 196. of the Geol. Society of Greece 34, S. 183-190.

GANAS, A. (2002): Methods for imaging earthquake deformation using satellite data and digital elevation models. In: Gorur, N., et al., (Eds.), Integration of Earth Science Research on the Turkish and Greek 1999 Earthquakes. Kluwer Academic Publishers, S. 197-204.

GillesPie, A.R.; KAHLE, A.B. \& WALKER, R.E. (1986): Color enhancement of highly correlated images. Decorrelation and HSI contrast stretches. Remote Sensing of Environment, V. 20, S. 209-235.

Goetz, A. F. H.; Billingsley, F. C.; Gillespie, A. R.; Abrams, M. J.; Squires, R. L.; Shoemaker, E. M.; LuChitTA, I. \& Elston, D. P. (1975): Application of ERTS images and image processing to regional geologic problems and geologic mapping in Northern Arizona. Jet Propulsion Lab. Tech. Report 32-1597, 188. 
GOLDSWORTHY, M. \& JACKSON, J. (2000): Active normal fault evolution in Greece revealed by geomorphology and drainage patterns, Geol. Society Journal, V. 157, S. 967-981.

GRIPPEN R. E. (1989): Selection of landsat TM band and band ratio combinations to maximize lithologic information in color composite. The 7th. Thematic Conference on Remote Sensing for Exploration Geology, Calgary, Alberta.

GuPTA, R. P. (2003): Remote sensing geology, 655 Seiten, Springer, New York.

HABERÄCKER, P. (1995): Praxis der Digitalen Bildverarbeitung und Mustererkennung, Hanser, München.

HAGG, W. (1998): Vorverarbeitung von Radarbilddaten. In: Bähr, H.P., Vögtle, T. (1998): Digitale Bildverarbeitung. Anwendung in Photogrammetrie, Karthographie und Fernerkundung. Heidelberg, S.264-299.

HAgEdORN, J. \& Poser, H. (1974): Räumliche Ordnung der rezenten geomorphologischen Prozesse und Prozesskombinationen auf der Erde.- In: Abh. Akad. Wiss. Göttingen, Math.-Physik. K1. III/29, Göttingen, S. 426-439.

Hansmann, B.C.; MeiJerink, A.M.J. \& KodituwakKu, K.A.W. (1992) An inductive approach for groundwater exploration. In: ITC Journal, V. 3, S. 269-276.

HEMPTON, M. (1985): Structure and deformation of the Bitlis suture near Lacke Haza, southeastern Turkey. Geol. Society of America Bulletin, V. 96, Nr. 2, S. 233-243.

HILDEBRANDT, R. R. (1992): Digitale und visuelle Interpretation von Landsat TM und SPOTDaten sowie Luftbildern in Hinblick auf die Geologie in der Region des Jebel Marra, Dissertation an der FU-Berlin, Fachbereich Geowissenschaften, Berlin.

HiLDEBRANDT, G. (1996): Fernerkundung und Luftbildmessung für Forstwirtschaft, Vegetationskartierung und Landschaftsökologie.- Wichmann Verlag, Karlsruhe.

HobBs, W.H. (1912): Earth Features and their Meaning. Macmillian Co., New York.

Hormann, K. (1971): Morphometrie der Erdoberfläche, Schr. Geogr. Inst., Univ. Kiel, 36, 178 S., Kiel.

HoRN, B.K.P., (1981): Hill shading and the reflectance map: Proceedings of the Institute of Electrical and Electronics Engineers, V. 69, Nr. 1, S. 14-47. 
JENSEN, J.R. (1996): Introductory digital image processing, Prentice-Hall, Englewood Cliffs, New Jersey, $318 \mathrm{~S}$.

Jenson, S. K. \& Domingue, J. O. (1988): Extracting topographic structure from digital elevation data for geographic information systems analysis. Photogrammetric Engineering and Remote Sensing. V. 54, Nr. 11, S. 1593-1600.

JONES, A.R. (1987): Landform investigation utilizing digitally processed satellite thematic mapper imagery. Earth, Moon and Planets, Springer Netherlands, V. 37, Nr. 2, S. 171185.

JORDAN, G., 2003. Morphometric analysis and tectonic interpretation of digital terrain data: A case study. Earth Surface Processes and Landforms 28: 807 - 822.

Jordan, G.; Csillag, G.; Szucs, A. \& Qvarfort, U. (2003): Application of digital terrain modelling and GIS methods for the morphotectonic investigation of the Kali Basin, Hungary. Zeitschrift Für Geomorphologie V. 47, S. 145-169.

Jordan, G.; Meijninger, B.M.L.; Van Hinsbergen, D.J.J.; MeulenkamP, J.E. \& VAn Dijk, P.M. (2005): Extraction of morphotectonic features from DEMs, development and applications for study areas in Hungary and NW Greece. In: International journal of applied earth observation and geoinformation: JAG, 7 (2005) 3, S. 163-182.

KAPPAS, M (1994): Fernerkundung nah gebracht. Leitfaden für Geowissenschaftler.

KATTAN, Z. (1997): Chemical and environmental isotope study of precipitation in Syria. Journal of Arid Environments V. 35, S.601-615.

KAUFMANN \& PFEIFFER (1986): Image optimization versus vlassification.- An application oriented comparison of different methods by use of Thematic Mapper data. In: DAMEN, M.C.J., SICCO SMIT, G. \& VERSTAPPEN, H.T. (eds.): Remote sensing for resources development and environmental management, 1, S. 31-35.

KAufMAnN, H. (1989): Mineral Exploration along the Aqaba-Levant Structure by use of TMdata: Concepts processing and results, Int. J. Remote Sens., V. 9, S. 1639-1658.

Keller, E.A., \& PinTER, N. (2002): Active tectonics: Earthquakes, uplift, and landscape: Upper Saddle River, Prentice Hall. 
KENEA, N. H. (1997): Improved geological mapping using Landsat TM data, Southern Red Sea Hills, Sudan: PC and IHS decorrelation stretching. - Int. J. remote Sens., V. 18, Nr. 6, S. 1233 - 1244, London.

KLIMASZEWSKI, M. (1982): Detailed geomorphological maps. ITC Journal, V. 3, S. 265-271.

KoIKe, K.; NAgAnO, S. \& KaWABA, K. (1998): Construction and analysis of interpreted fracture planes through combination of satellite image derived lineaments and digital elevation model data. Comput. Geosci. V. 24, S. 573-583.

KraAK, M. \& Ormelling, F. (2003): Cartography. Visualization of Spatial Data, 2nd edn, Pearson Education, Harlow, England.

KRAUS, K. (1990): Fernerkundung Bd.2: Auswertung photographischer und digitaler Bilder.313 S, Bonn (Dümmler).

KronberG, P. (1977): Die regionale und überregionale Bruchtektonik Mitteleuropas - Bestandsaufnahme und Interpretation. In: W. von Zeil (Ed.): Geotektonische Forschungen 53, S. 7-41. Stuttgart.

KronBerg, P. (1985): Fernerkundung der Erde - Grundlagen und Methoden des Remote Sensing in der Geologie.- 394 S., Stuttgart (Ferdinand Enke Verlag).

LATTMAN, L.H. \& PARIZEK, R.R. (1964): Relationship between fracture traces and the occurrence of groundwater in carbonate rocks. Journal of Hydrology 2: 73-91

LEE, J.S. (1980): Digital Image Enhancement and Noise Filtering by Use of Local Statistics, S. 165-168, IEEE Trans. Pattern Anal. Mach. Intelligence PAMI-2, No. 2.

LEEDER, M.R. \& JACKSON, J.A. (1993): The interaction between normal faulting and drainage in active extensional basins, with examples from western United States and central Greece. Basin Research, V. 5, S. 79-102.

Lidmar-Bergström, K.; Elvhage, C. \& Ringberg, B. (1991): Landforms in Skane, south Sweden. Geografiska Annaler. 73A, S. 61-91.

Lillesand, T.M. \& KiefER, R.W. (2000): Remote Sensing and Image Interpretation. 4. Aufl. New York.

LindsAy, J. B. (2005): The Terrain Analysis System: A tool for hydro-geomorphic applications. Hydrological Processes, 19: 1123-1130. 
LisT, F.K. (1969): Quantitative Erfassung von Kluftnetz und Entwässerungsnetz aus dem Luftbild. in: Bildmess. \& Luftbildwesen. V. 37, Nr. 4. S. 134-140, Karlsruhe.

List, F. K., Meissner, B. \& PöHLmann, G. (1990): Application of remote sensing and satellite cartography in preparing new geologic map 1 : 500,000. - In: Said, R., Hrsg.: Geology of Egypt, p. 27 - 44, Rotterdam (Balkema).

Maire, C. \& DATCU, M. (2004): Synergy of image and Digital Elevation Models (DEMs) information for Virtual Reality, Accessed on-line in September 2004: http://earth.esa.int/rtd/Events/ESA-EUSC_2004/Ar55_Maire.pdf

MARK, D.M. (1975): Geomorphometric parameters: A review and evaluation: Geografiska Annaler, 57A(3-4):165-177.

Miliaresis, G.C. \& ARgialas, D.P. (1999): Segmentation of physiographic features from the global digital elevation model/GTOPO30. Comput. Geosci. V. 25, S.715-728.

MiLIARESIS, G.C. (2001): Geomorphometric mapping of Zagros Ranges at regional scale. Comput. Geosci. V. 27, S. 775-786.

MoORE, I.D.; GraYson, R.B. \& LADSON, A.R. (1991): Digital terrain modelling: A review of hydrological, geomorphological and biological applications, Hydrological Processes, V. 5, S. 3-30.

Mulders, M.A., 1987: Remote Sensing in Soil Science, Amsterdam, Oxford, New York.

MuRPHY, W., (1993): Remote Sensing of active faults: case studies from southern Italy. Z. Geomorphol. N. F., Suppl. Bd. 94, S. 1-23.

O’Leary, D.W.; Freidman, J.D. \& Pohn, H.A. (1976): Lineament, linear, lineation: Some proposed new definitions for old terms. Geol. Society of America Bulletin, V. 87, S. 1463-1469.

Oguchi, T.; Aoki, T. \& MAtsutA, N. (2003): Identification of an active fault in the Japanese Alps from DEM-based hill shading. Computers \& Geosciences 29, 885-891.

OlAYA, V. (2004): A gentle introduction to SAGA GIS, Edition 1.1.

OnOrati, G.; Poscolieri, M.; Ventura, R.; Chiarini, V. \& Crucilla U. (1992): The digital elevation model of Italy for geomorphology and structural geology. Catena, V. 19, S. $147-178$.

PABOT, H. (1956): Rapport au gouvernement de Syrie sur I'ecologie vegetale et ses applications. FAO, Rome.

PASSARGE, S. (1921): Die Landschaft. Verlag von Quelle \& Meyers. 
PCIGEOMATICS, (16.11.2007): Zitieren aus dem World Wide Web. URL: HTTP://WWW.PCIGEOMATICS.COM/SERVICES/SUPPORT_CENTER/TECH_PAPERS/GRSG_ASTER_ARTICLE.PDF.

PENCK, A. (1894): Morphologie der Erdoberfläche, Stuttgart, Engelhorn. 2v.

PIKE, R. (1992): Machine Visualization of Synoptic Topography by Digital Image Processing. US Geol. Survey Bulletin 2016: B1- B12.

PIKE, R. (2000): Geomorphometry-diversity in quantitative surface analysis. Progress in Physical Geography, V. 24, S. 1-20.

Ponikarov. V., Shatsky, V. \& Kazmin. V. (1963): Geological Map of Syria 1:200.000 and Explanatory Notes, Technoexport, Moscow.

Prost, G. L. (1994): Remote Sensing for Geologist: A guide to Image Interpretation. Gordon and Breach Science publishers.

RichARDS, J.A. \& JIA X. (1999): Remote Sensing Digital Image Analysis: An Introduction. (Third Edition). NewYork: Springer-Verlag.

RITTER, C. (1817): Die Erdkunde. I.

ROESNER, U. (1995): Zur quartären Landschaftsentwicklung in den Trockengebieten Syrien, Relief,Boden,Paläoklima 10, Bornträger, Berlin.

Roland, N.W. (1973): Die Anwendung der Photointerpretation zur Lösung stratigraphischer und tektonischer Probleme im Bereich von Bardei und Aozou (Tibesti-Gebirge, Zentral Sahara). Berl. geowiss. Abh. 19, S. 1-80.

RosenthaL, E. (1987): Chemical composition of rainfall and groundwater in recharge areas of Bet Shean-Harod multiple Grundwasserleiter system, Israel. J Hydrology, V. 89, S. $329-352$

Rother, K. (1993): Der Mittelmeerraum. Stuttgart.

SABINS, F.F. (1997): Remote sensing. Principles and interpretations.

SALVI, S. (1995): Analysis and interpretation of Landsat synthetic stereo pair for the detection of active fault zones in the Abruzzi Region (Central Italy). Remote Sens. Environ. V. 53, S. $153-163$.

SANDER, P. (1996): Remote Sensing and GIS For Groundwater Assessment in Hard Rocks: Applications to Water Well Siting in Ghana and Botswana. PhD dissertation, Chalmers University of Technology, Sweden, Publ. A 80.

SCHOWENGERDT, R.A. (1997): Remote Sensing: models and methods for image processing. 2. Aufl., San Diego. 
SELKHOZPROMEXPORT (1979-1982): Hydrogeological and hydrological investigation in four areas of Syrian Arab Republic, Map 1:500.000, USSR Selkhozpromexport Gruzgiprovodkhoz.

Siegal, B.S. \& Gillespie, A.R. (1980): Remote Sensing in Geology. John Wiley and Sons, New York.

Silverman, B. W. (1986): Density Estimation for Statistics and Data Analysis Chapman and Hall, London.

SimPSON, D.W. \& ANDERS, M.H. (1992): Tectonics and topography of the Western United States an application of digital mapping. GSA Today 2, 118-121.

SINGH, A. \& HARRISON, A. (1985): Standardised principal components. International Journal of Remote Sensing, V. 6, Nr. 6, S. 883-396.

SMITH, M.J. \& ClARK, C.D. (2005): Methods for visualisation of high resolution digital elevation models for landform mapping. Earth Surface Processes and Landforms, V. 30, S. $885-900$.

StRAHLER, A. N. (1952): Dynamic basis of geomorphology: Geol. Society of America, V. 63, S. 923- 938.

Thelin, G.P. \& PIKE, R.J. (1991): Landforms of the conterminous United States -A digital shaded-relief portrayal: U.S. Geol. Survey Miscellaneous Investigations Map I-2206, scale $1: 3.500 .000$.

VAN LIERE, W. J. (1960-1961): Observations on the Quaternary of Syria. Publication of the General Directorate of Antiquites and Museums in the Syrian Arab Republic, S. 5-69.

VAuMAS, E. de (1957): Plateaux, plaines et dépressions de la Syrie intérieure septentrionale (du paralléle d'Alep au paralléele de Homs): Étude morphologique. Bull, Soc. Géogr. Egypte, V. 30, S. 97-235.

VAuMAS, E. de (1961): Structure et morphologie du Proche-Orient. Nouvel essai de synthèse et orientation de recherche.

Verstappen, H. T. (1977): Methodische Probleme der geomorphologischen Kartierung mittels Fernerkundung. Geomethodica, V. 2, S. 19-43.

VINCENT, K.R. (1997): Fundamentals of geological and environmental remote sensing, London.

VoÛTE, C. (1955): Climate or tectonics? Some remarks on the evolution of the valley of the Orontes (Nahr el Aassi) between Homs and the marshy plains of the Ghab (Syria). Geologie en Mijnbouw. Nieuwe série, 17e Jaargang, S. 197-206. 
Vysotsky, E.M.; VishneVsKayA, E.A. \& ElobogoeV, A.V. (2002): Neotectonic analysis of northern Lake Teletskoe using digital elevation modeling. Geologiya i Geofizika, V. 43, S. $1099-1106$.

WAGNeR, W. \& GEYH, M. A. (2000): Application of Environmental Isotope Methods for Groundwater Studies in the ESCWA Region. - Geol. Jb., Hannover.

WALLEY, C. D. (1988): A braided strike-slip model for the northern continuation of the Dead Sea Fault and its implications for Levantine tectonics. Tectonophysics, V. 145, S. 63-72.

WDOWINSKI, S. \& ZiLbeRMAN, E. (1997): Systematic analyses of the large-scale topography and structure across the Dead Sea Rift. Tectonics, V. 16, S. 409- 424.

Weibel, R. \& Heller, M. (1991): Digital terrain modeling. In: Maguire, D. J., Goodchild, M. F. und Rhind, D. W. (Hrsg.): Geographical information systems: principles and applications. Longman, London.

Williams, R. S. (1983): Geological Application, in: Colwell, R. N.: Manual of Remote Sensing, V. II, 2nd Ed., S. 1869-1878, American Society of Photogrammetry, Falls Church, Virginia, S. 1667-1935.

WIRTH, E. (1971): Syrien, eine geographische Landeskunde, Wissenschaftliche Länderkunden, Band 4/5, Wissenschaftliche Buchgesellschaft, Darmstadt.

Wolfart, R. (1967): Geologie von Syrien und dem Libanon. Beitr. Zur regionalen Geologie der Erde, 6. Berlin.

ZANChi, A.; Crosta, G.B. \& Darkal, A.N. (2002): Paleostress analyses in NW Syria: Constraints on the Cenozoic evolution of the northern margin of the Arabian Plate. In: Tectonophysics, V. 357, S. 255-278.

ZumsPreKel, H. \& PRINZ, T. (2000): Computer enhanced multispectral remote sensing data: a necessary tool for the geological mapping of Archean terrains in (semi)arid environments.- Computer and Geosciences, V. 26, S. 87-100. 

Anhang 


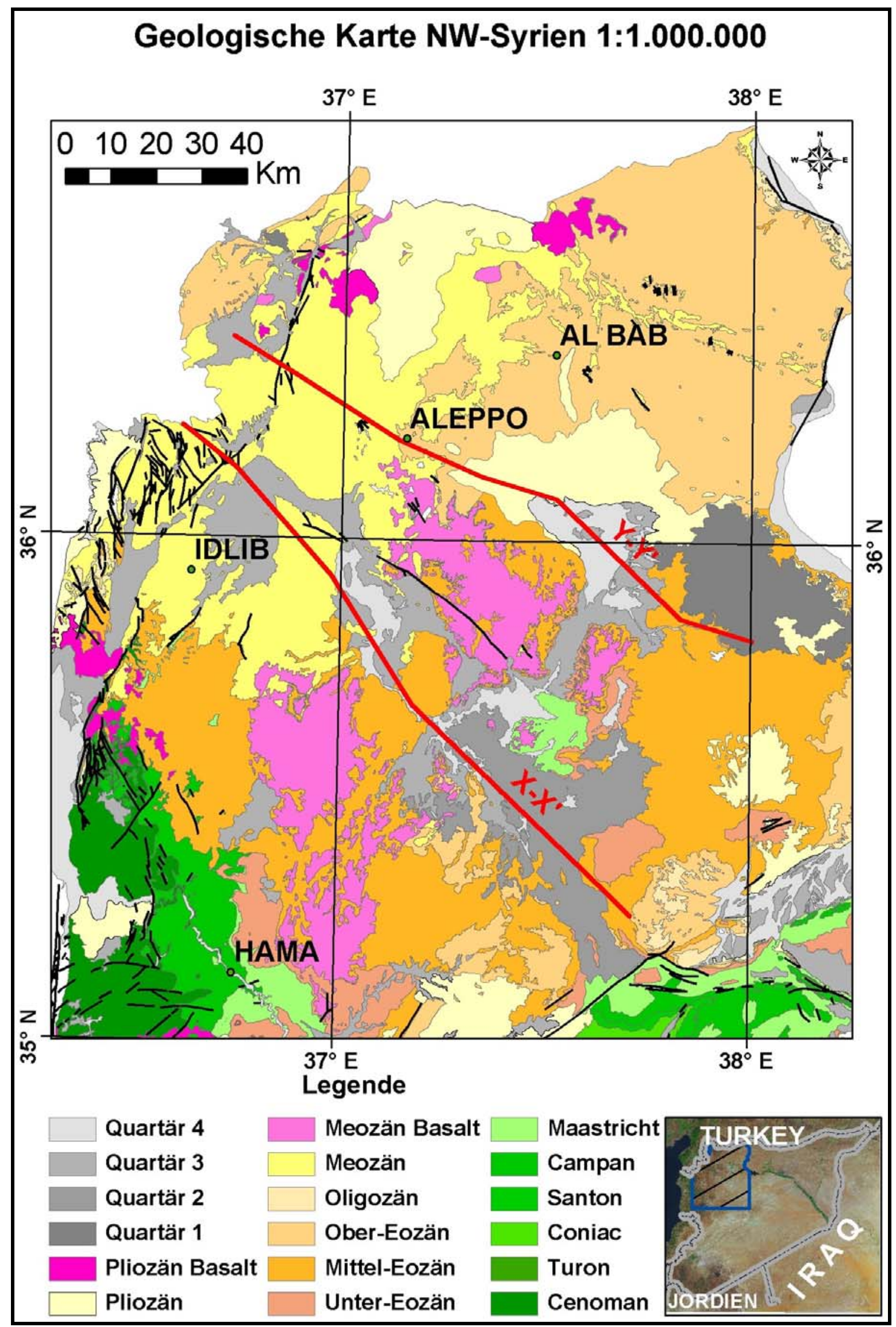

Anhang 1: Übersichtkarte der Geologie des betrachteten Gebiets - 171 - 


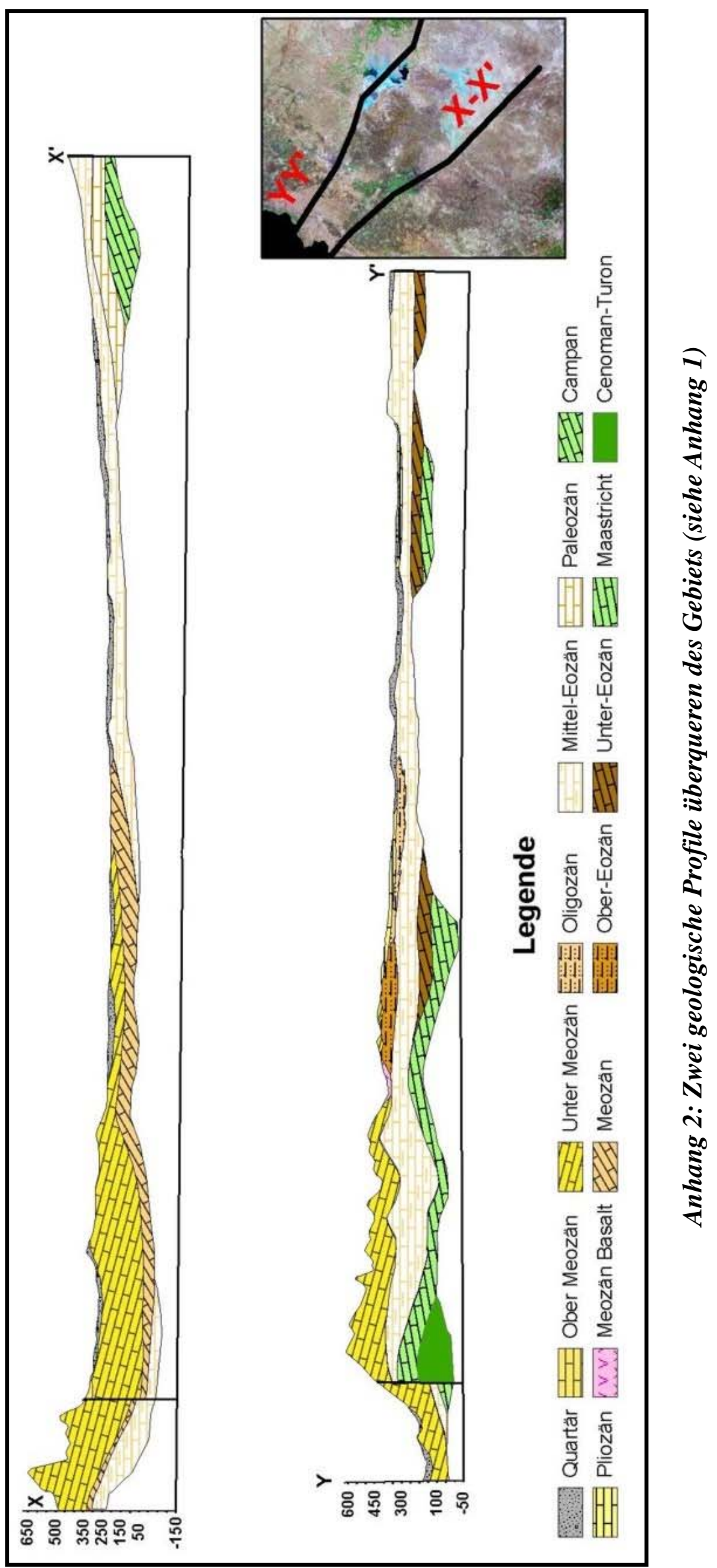




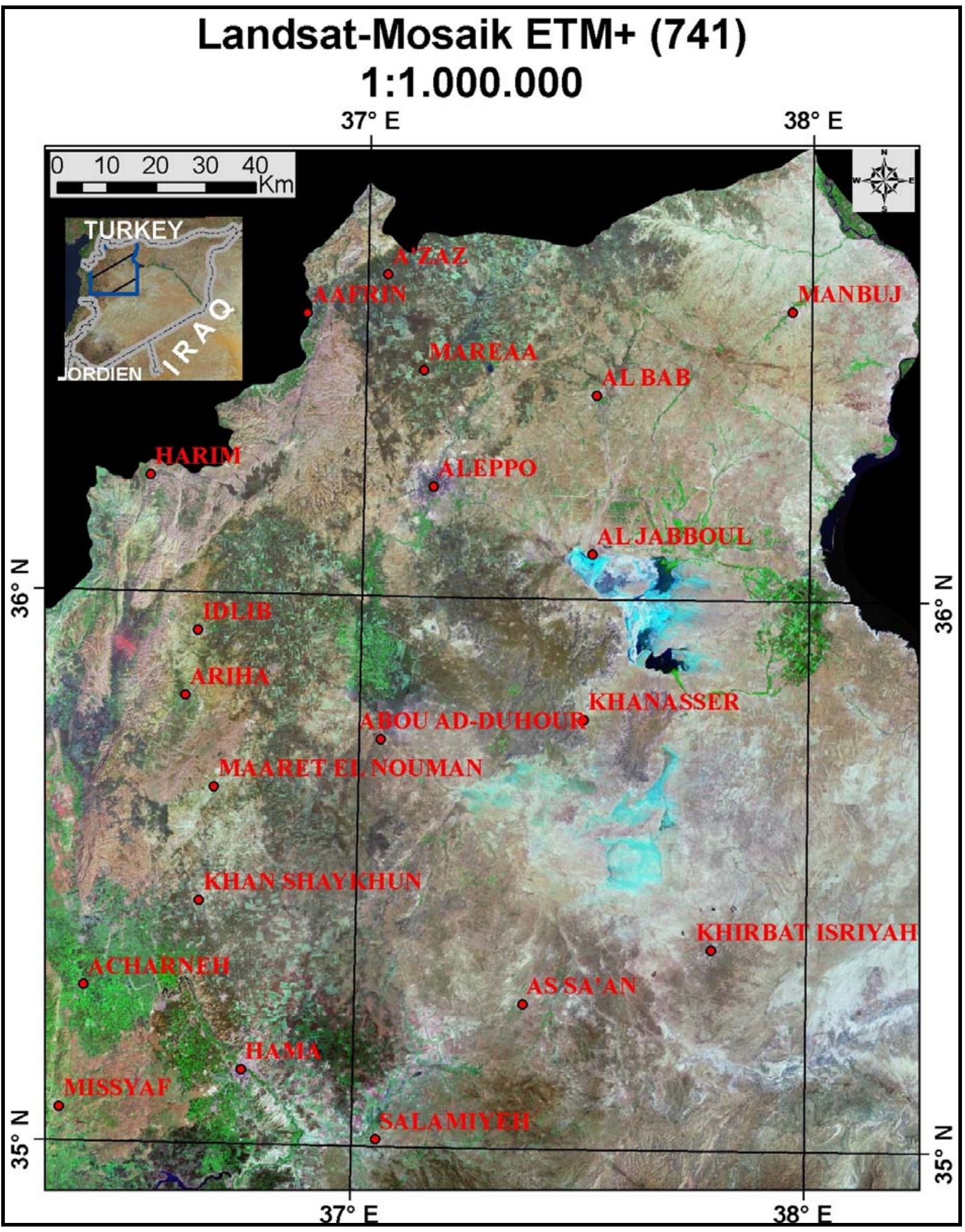

Anhang 3: Landsatmosaik mit wichtigen Ortsnamen 


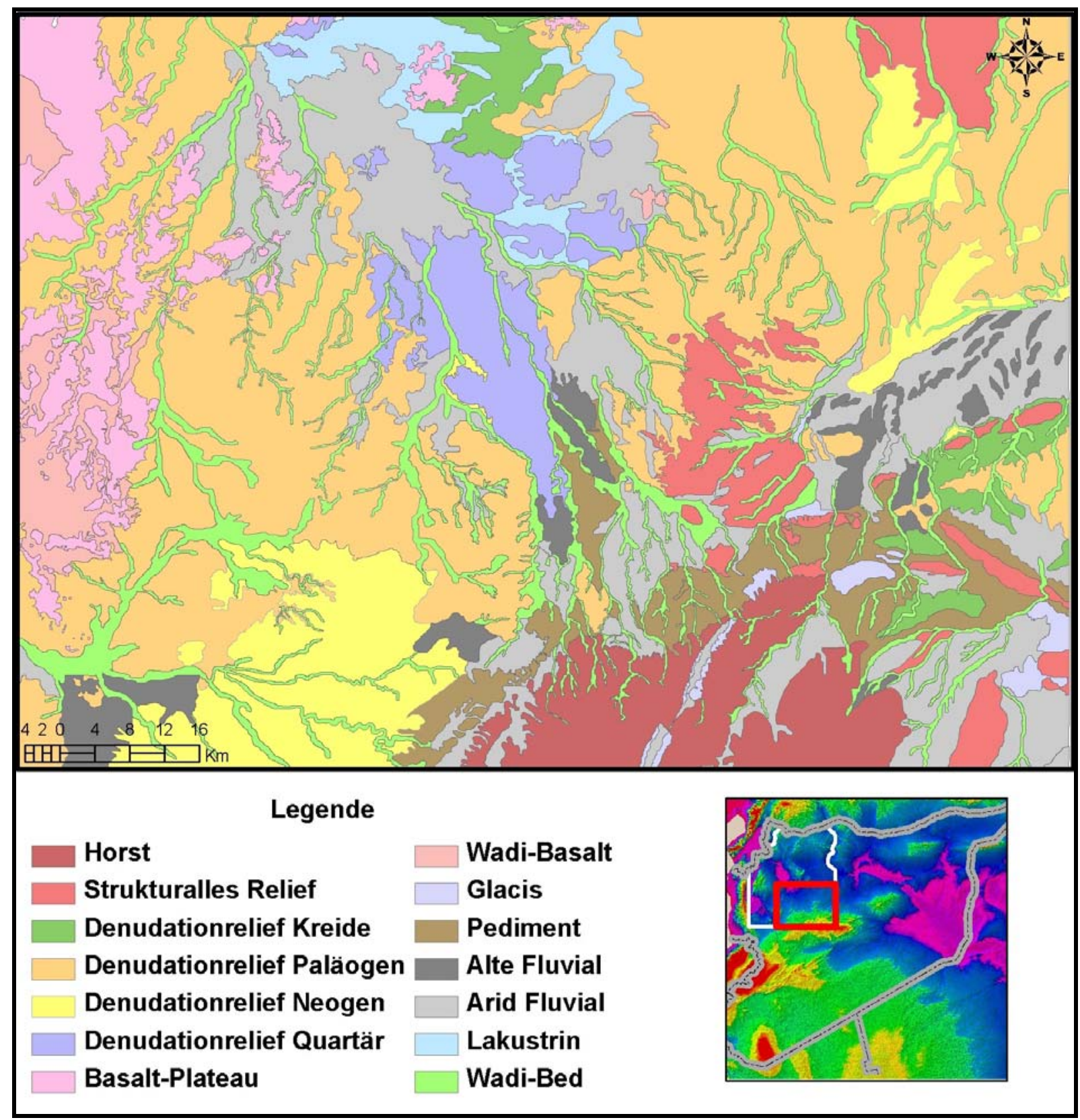

Anhang 4: Geomorphologische Karte des hydrogeomorphologisch betrachteten Gebiets 


\section{Danksagung}

Die vorlegende Doktorarbeit wurde am Geowissenschaftlichen Zentrum (GZG) der Universität Göttingen angefertigt. Für den erfolgreichen Abschluss dieser Arbeit schulde ich vielen Menschen meinen herzlichen Dank. An erster Stelle möchte ich mich ganz besonders bei meinen Doktorvater Professor Dr. M. Kappas und Professor Dr. M. Sauter recht herzlich für die Unterstützung des Themas und für die wichtigen Hinweise zum Aufbau der Arbeit bedanken. Sie haben mir viel Geduld und Vertrauen entgegengebracht und mich in schwierigen Situationen mit ihrer Diskussionsbereitschaft und ihren wertvollen Ratschlägen zum Weitermachen motiviert. Sie haben mich bestärkt, wenn ich selbst an der erfolgreichen Fertigstellung meiner Doktorarbeit gezweifelt habe.

Großen Dank schulde ich meinem ehemaligen Betreuer Professor Dr. D. Helmcke, der mich zu dieser Arbeit zu Beginn motiviert und mich bei meinen ersten Schritten begleitet hat. Leider ist er zu früh verstorben. An dieser Stelle möchte ich auch den Professoren am GZG vor allem Prof. Dr. H. Porada, Prof. Dr. J. Schneider, Prof. Dr. H. Ruppert, Prof. Dr. M. Kuhle und Prof. Dr. K. H. Pörtge für ihre freundliche Unterstützung bei meinen ersten Schritten im GZG danken.

Ein besonderes Dankeswort gilt auch meinen Kollegen am Geowissenschaften Wissenschaftszentrum (GZG), speziell Dr. T. Heinrich, Dr. B. Wagner, Dr. S. Erasmi, Dipl. Geogr. O. Conrad, Dipl. Phys. A. Ringeler und Dipl.-Geol. M. Toll, die mich bei zahlreichen Diskussionen unterstützt und damit wichtige wertvolle Anregungen für meine wissenschaftliche Arbeit geliefert haben. Für die sorgfältigen Laboruntersuchungen meiner Proben und betreffender Diskussionen bedanke ich mich bei den Kollegen Dr. T. Licha und Frau M. Rittmeier. Bedanken möchte ich mich auch bei meinen Kollegen im Institut, den Herren Dr. C. Noubactep, T. Lange, F. Deisinger, T. Geyer, M. Röhring und der Frau D. Friedt sowie Frau K. Thomas für ihre freundliche Unterstützung und ihren Zuspruch in langen privaten Gesprächen.

Den Fachleuten und Zuständigen an der Bundesanstalt für Geowissenschaften und Rohstoffe (BGR) sei herzlich .gedanktEin besonderer Dank geht an den Herrn Dr. Dieter Plöthner, der mir bei meinen Anfragen stets freundlich und kompetent weiterhelfen konnte.

Ein herzliches Dankeschön gilt meiner Familie für Ihre unermüdliche Geduld während meiner wissenschaftlichen Arbeit. Sie haben mir den Rücken freigehalten, damit ich mich ganz auf meine wissenschaftliche Arbeit konzentrieren konnte. 


\section{Lebenslauf}

Name: $\quad$ Wahib Sahwan

Geburtsdatum: $\quad$ 18.02.1974

Geburtsort: $\quad$ Taybat Al Imam / Hama

Staatsangehörigkeit: syrisch

Familienstand: verheiratet

\section{$\underline{\text { Schulabschluss }}$}

05. 1993

Abitur, Gymnasium Taybat Al Imam/Hama - Syrien

\section{$\underline{\text { Studium }}$}

$1993-1997$

BSc. Of Geology - Universität Aleppo in Aleppo/Syrien - Fakultät für Wissenschaften (Abteilung Geologie) - Hochschulabschluss (Sehr Gut).

\section{Wissenschaftlicher Werdegang}

Seit 10. 1998 Wissenschaftlicher Angestellter der Abteilung Geologie, Fakultät für Wissenschaften der Universität Aleppo.

04. 2002 - 02. 2008 Promotionsarbeit zum Thema „Geomorphologische Untersuchungen mittels GIS- und Fernerkundungsverfahren unter Berücksichtigung hydrogeologischer Fragestellungen - Fallbeispiele aus Nordwest Syrien -“

\section{Stipendium}

02.01 bis $02.08 \quad$ Promotionsstipendium der Universität Aleppo/Syrien.

\section{Studienbegleitende Tätigkeiten}

Seit 04.2006 Wissenschaftliche Hilfskraft in der Abteilung Angewandte Geologie, Geowissenschaftliches Zentrum Göttingen, GeorgAugust-Universität Göttingen. 